\title{
High Harmonic Generation And ION ACCELERATION WITH High-Intensity LASER Pulses
}




\section{Samenstelling van de promotiecommissie:}

\section{Voorzitter \& secretaris:}

Prof. dr. M. J. Peters

University of Twente, The Netherlands

\section{Promotor:}

Prof. dr. K.-J. Boller

University of Twente, The Netherlands

\section{Leden:}

Prof. dr. F. Bijkerk

University of Twente, The Netherlands

Prof. dr. J. L. Herek

Prof. dr. H. Zacharias

University of Twente, The Netherlands

Dr. Ph. Martin

Westfälische Wilhelms-Universität Münster,

Germany

CEA Saclay-DSM/IRAMIS/SPAM, France

The research presented in this thesis was carried out at the Laser Physics and Nonlinear Optics group, Department of Science and Technology, MESA ${ }^{+}$ Institute of Nanotechnology, University of Twente, P.O. Box 217, $7500 \mathrm{AE}$ Enschede, The Netherlands, and at CEA-Saclay, DSM/IRAMIS/SPAM, 91191 Gif sur Yvette, France. The work was financially supported by the Dutch Ministry of Education, Culture and Science (OC\&W) and partly by the European Concerted Research Action COST P14 and by Laserlab Europe.

Copyright (c) 2009 by Rolf A. Loch

ISBN: 978-90-365-2882-5

Printed by Ipskamp Drukkers B.V., Enschede, The Netherlands 


\section{High Harmonic Generation And ION ACCELERATION WITH High-Intensity Laser Pulses}

\section{ProefsChRIFT}

ter verkrijging van

de graad van doctor aan de Universiteit Twente, op gezag van de rector magnificus, prof. dr. H. Brinksma,

volgens besluit van het College voor Promoties in het openbaar te verdedigen

op vrijdag 18 september 2009 om 16.45 uur

door

Rolf Antonie Loch

geboren op 8 juli 1981

te Apeldoorn 
Dit proefschrift is goedgekeurd door de promotor

Prof. dr. K.-J. Boller 
To my parents and brother,

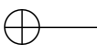


$\bigoplus$ 


\section{Publications}

- R. A. Loch, A. Levy, T. Ceccotti, F. Quere, C. Thaury, H. George, F. Bijkerk, K.-J. Boller, and Ph. Martin, "Enhanced ion acceleration with extremely thin foils," The European Physical Journal Special Topics 175, 133-138 (2009).

- R. A. Loch, Ph. Martin, T. Ceccotti, P. Monot, F. Quere, H. George, M. Bougeard, F. Reau, P. D'Oliveira, and K.-J. Boller, "High-order harmonic and fast ion generation in high intensity laser-solid interactions," in Laser-Driven Relativistic Plasmas Applied to Science, Industry and Medicine, the Second International Symposium, Kizugawa City, Japan, 2009, accepted for publication in Conference Proceedings.

- C. Thaury, H. George, F. Quere, R. Loch, J. P. Geindre, P. Monot, and Ph. Martin, "Coherent dynamics of plasma mirrors," Nature Physics 4, 631634 (2008).

- A. Andreev, A. Levy, T. Ceccotti, C. Thaury, K. Platonov, R. A. Loch, and Ph. Martin, "Fast-Ion Energy-Flux Enhancement from Ultrathin Foils Irradiated by Intense and High-Contrast Short Laser Pulses," Physical Review Letters 101, 1550024 (2008).

- R. A. Loch, C. Thaury, H. George, A. Levy, F. Quere, T. Ceccotti, A. Andreev, K. Platonov, J.-P. Geindre, P. Monot, Ph. Martin, F. Bijkerk, and K.-J. Boller, "Extreme nonlinear optics: laser-accelerated ion beams and coherent ultrashort x-ray pulses," Physics@FOM Veldhoven conference, Veldhoven, The Netherlands, 20-21 January 2009, oral presentation.

- R. A. Loch, C. Thaury, H. George, A. Levy, F. Quere, T. Ceccotti, A. Andreev, K. Platonov, J.-P. Geindre, P. Monot, Ph. Martin, F. Bijkerk, and K.-J. Boller, "Laser based beams of ions and coherent x-rays," MESA $^{+}$Meeting 2008, Enschede, The Netherlands, 23 September 2008, oral presentation.

- R. A. Loch, M. W. Hendrikx, H. M. J. Bastiaens, A. Irman, M. J. H. Luttikhof, F. A. van Goor, K.-J. Boller, "Enhancement of High Harmonic Generation from Gases," ICFO The Institute of Photonic Sciences, Castelldefels (Barcelona), Spain, 2 September 2008, oral presentation. 
- B. Zandt, M. W. Hendrikx, R. A. Loch, H. M. J. Bastiaens, A. Irman, M. J. H. Luttikhof, F. A. van Goor, A. G. Khachatryan, F. Bijkerk and K.-J. Boller, "Enhancement of high harmonic generation in a helium-xenon gas mixture," $21^{\text {st }}$ NNV-symposium on Plasma Physics and Radiation Technology, Lunteren, The Netherlands, 3-4 March 2009, poster contribution.

- R. A. Loch, A. Levy, T. Ceccotti, F. Quere, C. Thaury, H. George, F. Bijkerk, K.-J. Boller, and Ph. Martin, "Extremely thin foils for enhanced ion acceleration," $20^{\text {th }}$ NNV-Symposium on Plasma Physics and Radiation Technology, Lunteren, The Netherlands, 4-5 March 2008, poster contribution.

- M. W. Hendrikx, R. A. Loch, H. M. J. Bastiaens, A. Irman, M. J. H. Luttikhof, F. A. van Goor, A. G. Khachatryan, P. J. M. Peters, F. Bijkerk and K.-J. Boller, "Enhanced high harmonic generation from gas mixtures," $20^{\text {th }}$ NNV-Symposium on Plasma Physics and Radiation Technology, Lunteren, The Netherlands, 4-5 March 2008, poster contribution.

- M. J. H. Luttikhof, A. Irman, W. O. Rekers, A. G. Khachatryan, F. A. van Goor, R. A. Loch, J. W. J. Verschuur, H. M. J. Bastiaens, F. Bijkerk, and K.-J. Boller, "Recent progress of theory and experiments on laser wakefield acceleration at the University of Twente," $20^{\text {th }} \mathrm{NNV}$ Symposium on Plasma Physics and Radiation Technology, Lunteren, The Netherlands, 4-5 March 2008, poster contribution.

- R. A. Loch, A. Levy, T. Ceccotti, F. Quere, C. Thaury, H. Lagadec, F. Bijkerk, K.-J. Boller, and Ph. Martin, "Enhanced Ion Acceleration with Extremely Thin Foils," $1^{\text {st }}$ International Conference on Ultra-intense Laser Interaction Sciences, Bordeaux, France, 1-5 October 2007, poster contribution.

- R. A. Loch, M. J. H. Luttikhof, M. W. Hendrikx, A. Irman, A. G. Khachatryan, F. A. van Goor, H. M. J. Bastiaens, P. J. M. Peters, F. Bijkerk, and K.-J. Boller, "Ultra-high Intensity Laser-Plasma Interactions," $1^{\text {st }} \mathrm{In}$ ternational Conference on Ultra-intense Laser Interaction Sciences, Bordeaux, France, 1-5 October 2007, poster contribution.

- R. A. Loch, M. J. H. Luttikhof, M. W. Hendrikx, A. Irman, A. G. Khachatryan, F. A. van Goor, H. M. J. Bastiaens, P. J. M. Peters, F. Bijkerk, and K.-J. Boller, "High energy particles and coherent X-rays for high resolution observations," MESA ${ }^{+}$Meeting 2007, Enschede, The Netherlands, 11 September 2007, poster contribution.

- R. A. Loch, F. Quere, C. Thaury, H. Lagadec, A. Levy, T. Ceccotti, F. Bijkerk, K.-J. Boller, and Ph. Martin, "Novel Observations on HighIntensity Laser-Plasma Interactions," Workshop Emerging Sources, Lund, Sweden, 11-13 June 2007, poster contribution. 
This thesis reports on research performed on high harmonic generation and ion acceleration, processes that are based on the interaction of gaseous and solid media with high-intensity laser pulses. High harmonic generation leads to the production of beams of ultrashort, laser-like radiation in the wavelength range reaching from the extreme ultraviolet (XUV) to soft X-rays. Ion acceleration generates highly energetic protons and ions in the form of highly directional and pulsed beams with an ultrashort duration.

The generation of such beams of accelerated particles and X-ray radiation so far requires large facilities such as synchrotrons, linear particle accelerators and free electron lasers for research and applications in materials science, condensed matter physics, biology and medicine among others. The main problem with these facilities is that their size makes them rather exclusive and access to them is limited. This impedes progress in the associated fields of research and applications. The high potential of high-intensity lasers is that they can deliver accelerated particles and X-ray radiation as well and provide improved output parameters but via a much more compact approach. This would significantly advance research and open the way to widespread applications.

However, to fully enable applications of ion acceleration and high harmonic generation, both these laser-driven processes require an enhancement of the flux and maximum energy of the accelerated ions and the harmonic radiation. The scope of this thesis is to present novel investigations and methods towards achieving this goal via an improved understanding of the underlying physics and, to provide a more complete identification of the actual requirements of the laser and the target for optimum output.

The first part of this work concerns high harmonic generation in gaseous atomic media, using laser intensities that are sufficiently high to induce tunnelionization of electrons. The ionized electrons make an excursion and are accelerated under the influence of the strong electric field of the laser. Some electrons return to their parent ion and, upon recombination, emit high-frequency radiation. The radiation spectrum contains high harmonics and shows a maximum frequency in the form of a cut-off.

We constructed and tested a setup to generate high harmonics for use with the terawatt laser at the Laser Physics and Nonlinear Optics group to provide the preconditions for future exploration of several novel methods to enhance the efficiency and cut-off of high harmonic generation. The setup employs a 
capillary waveguide because such a configuration results in a higher output and a higher degree of temporal and spatial coherence compared to gas jets. Furthermore, with a capillary waveguide additional enhancement methods can be implemented or investigated in novel parameter regimes, for example, quasiphase-matching of high harmonic generation in partially ionized gases prepared in a discharge. We implemented a novel manner of differential pumping by introducing additional outlet slits in the capillary to reduce re-absorption of the high harmonics behind the interaction region. With an XUV-CCD camera and a movable transmission grating we were able to conveniently record both the harmonic spectrum and the beam profile.

As a first experimental test, harmonic spectra from xenon and argon were recorded which were found to be fully consistent with previously reported results. In the next step we performed a novel demonstration experiment to extend a previously shown enhancement technique called Harmonic Excitation. The basic working of this technique is that atoms are first excited with XUV radiation before the infrared drive laser radiation is applied. The XUV radiation facilitates tunnel-ionization by the drive laser so that the efficiency of high harmonic generation is increased. We demonstrated such an enhancement by harmonic excitation for the first time in a guided-wave geometry. This prepares the extension of this approach towards the generation of significantly shorter wavelengths in future work based on mixtures of ions from a capillary discharge.

The second part of the research described in this thesis concerns ion acceleration and high-order harmonic generation from solid-state targets. Each process has its own requirements for various properties of the target and the used laser pulses, e.g., the pulse intensity, the duration and the temporal contrast. With high-intensity laser pulses with an ultra-high contrast both processes may simultaneously appear because the two seemingly different processes are based on the same type of initial electron dynamics. When the high-intensity laser pulse arrives at the target the solid becomes almost instantaneously and fully ionized, this results in a dense and reflective plasma. The interaction of this plasma with the laser pulse leads to the heating of some part of the free plasma electrons to relativistic energies. In the following steps of the dynamics these electrons are responsible for the generation of high-order harmonics or fast ions, depending on the laser and target parameters.

Two mechanisms for harmonic generation had previously been identified and have been under investigation in this thesis for an improved understanding and optimization. The first mechanism is described by the Relativistic Oscillating Mirror model. High-order harmonics are generated via extremely strong Doppler shifts occurring upon reflection of the incident laser light off relativistic electrons moving out of the plasma into the vacuum towards the laser pulse. The second mechanism is based on the laser-driven return of the relativistic electrons into the remaining dense plasma. There, the returning electrons excite collective and coherent wake oscillations of the plasma. These 
plasma oscillations emit radiation in the form of high-order harmonics. This process is called Coherent Wake Emission.

The electron dynamics leading to ion acceleration is similar but can be optimized using a thin foil as the target. If the foil possesses a sufficiently small thickness, part of the highly energetic electrons move through the target, leave the target through its rear surface and form a dense sheath behind the rear surface. The sheath generates a large field that accelerates ions from the rear surface of the foil to $\mathrm{MeV}$ energies. This process is called Target Normal Sheath Acceleration.

For novel experimental investigations on ion acceleration and high-order harmonic generation using solid targets, a laser with state-of-the-art qualifications, i.e. with relativistically high intensity and record-breaking ultra-high pulse contrast, at the Physique à Haute Intensité group has been made available in a close collaboration. Special attention was given to the preparation and investigation of extremely thin foils, i.e. freestanding nanofoils, to enter a very promising, but largely unexplored, regime. This regime of laser-matter interaction, called the transparent regime where the plasma thickness is comparable to the penetration depth of the laser light (a few nanometers), had only been theoretically investigated to this point. An attractive prediction is that moving into this regime would result in an enhanced maximum energy and an enhanced number of accelerated ions. However, these predictions were obtained by neglecting a most important physical effect, namely the early expansion of the plasma before the drive pulse reaches its peak intensity.

In order to test the validity of these predictions, we experimentally investigated ion acceleration in this transparent regime for the first time using the extremely thin targets (nanofoils) and the high-intensity laser pulses of ultrahigh contrast and, compared these results with data from improved numerical modeling. We also investigated for the first time high-order harmonic generation in the transparent regime experimentally and via numerical modeling. From our experiments and numerical modeling can be concluded that it is important to take into account the limited expansion of the target caused by the pedestal of the ultra-high contrast laser pulse. Furthermore, in order to reach the theoretically interesting regimes of ion acceleration and harmonic generation, in experiments a further improved pulse contrast is required at the picosecond time scale. Further improvements can be expected if freestanding nanofoil targets with a higher damage threshold can be realized in order to reduce the pre-expansion of the target.

In the course of these experiments we also performed novel investigations with thick, bulk targets. We verified that the high-order harmonic radiation generated via coherent wake emission is spatially coherent and, that the optical phase of the XUV emission can be controlled via the drive laser intensity. In these experiments we generated three closely spaced foci of the drive laser with adjustable intensities. In this way we realized three mutually synchronized XUV sources on the target surface, so that an interference pattern could be observed in the XUV far-field. These interference patterns also allowed the 
study of the dynamics of the plasma electrons during the interaction with the laser. This is of great importance for understanding more of the physical details of high-intensity laser-matter interactions, such as the absorption of light in overdense plasmas at relativistic intensities and the efficient generation of beams of highly energetic particles and coherent X-rays. 


\section{Samenvatting}

Dit proefschrift beschrijft het onderzoek naar hoge harmonische generatie en ionenversnelling, processen die gebaseerd zijn op de interactie van gassen en vaste stoffen met laserpulsen met een hoge intensiteit. Hoge harmonische generatie leidt tot de productie van een stralenbundel van extreem ultraviolet (XUV) tot zachte röntgenstraling met laser-achtige eigenschappen en van ultrakorte tijdsduur. Ionenversnelling genereert uiterst energetische protonen en ionen in een scherp gerichte bundel van ultrakorte tijdsduur.

Tot dusver zijn voor de generatie van dergelijke bundels van versnelde deeltjes en röntgenstraling grootschalige faciliteiten vereist, zoals synchrotronen, lineaire deeltjesversnellers en vrije-elektronenlasers die gebruikt worden voor onderzoek en toepassingen in bijvoorbeeld materiaalkunde, vaste stof fysica, biologie en geneeskunde. Het belangrijkste probleem met deze faciliteiten is dat er wereldwijd maar een beperkt aantal van bestaan en dat de mogelijkheid voor experimenten beperkt is. Dit verhindert de voortgang van onderzoek en de verspreiding van toepassingen. Echter, lasers met hoge intensiteit zijn een veelbelovend alternatief omdat hiermee ook versnelde deeltjes en röntgenstraling geproduceerd kunnen worden met betere eigenschappen en via een compactere methode. Dit zou een opmars van het onderzoek naar en toepassingen van dit soort bundels mogelijk maken.

Echter, voor de toepassing van de laser-gedreven ionenversnelling en hoge harmonische generatie is een verhoging van de maximum energie en het aantal versnelde ionen en harmonische fotonen vereist. In dit proefschrift wordt onderzoek gepresenteerd naar nieuwe methodes om dit doel te bereiken via een verbeterd begrip van de achterliggende fysica, en wordt een vollediger beeld verschaft van de daadwerkelijke eisen die aan de laser en de materie worden gesteld voor de optimalisatie van de opbrengst.

Het eerste deel van dit proefschrift behandelt hoge harmonische generatie in gasvormige atomaire media met gebruikmaking van laserintensiteiten die voldoende hoog zijn om tunnel-ionisatie van elektronen te veroorzaken. De geïoniseerde elektronen worden versneld door het sterke veld van de laser. Sommige elektronen keren terug naar hun oorspronkelijke ion en zenden vervolgens kortgolfige straling uit tijdens de recombinatie. Het stralingsspectrum bevat hoge harmonischen en heeft een duidelijk maximum in de frequentie (minimum golflengte).

We hebben een opstelling opgebouwd en getest om hoge harmonischen te 
genereren en om nieuwe methodes te kunnen onderzoeken die de efficiëntie en de maximumfrequentie van de geproduceerde straling verhogen. Hierbij maken we gebruik van de terawatt laser van de vakgroep Laserfysica en Nietlineaire Optica. De opstelling bevat een capillaire golfgeleider waarmee een verhoogde opbrengst en coherentie kan worden bereikt. Het gebruik van een capillair maakt het bovendien mogelijk om extra technieken te implementeren en nieuwe parameter regimes te onderzoeken, zoals "quasi-phase-matching" in gedeeltelijk geïoniseerde gassen die aangemaakt zijn met een capillaire ontlading. We hebben een nieuwe manier van differentiëel pompen ingevoerd om absorptie van de harmonische straling achter het capillair te verminderen. Met een XUV-CCD camera en een verplaatsbaar transmissietralie kunnen we gemakkelijk zowel het harmonische spectrum als het bundelprofiel meten.

Als eerste experimentele test zijn harmonische spectra van xenon en argon gemeten die in overeenstemming zijn met eerder gepubliceerde resultaten. In de volgende stap hebben we een nieuw demonstratie experiment uitgevoerd om een bekende verbeteringstechniek, Harmonische Excitatie, uit te breiden. De fundamentele werking van deze techniek berust daarop dat elektronen eerst worden aangeslagen met XUV straling voordat de infrarode aandrijvingslaser wordt aangeboden. De XUV straling vergemakkelijkt de ionisatie met de laser zodat de efficiëntie van hoge harmonische generatie verhoogd wordt. We hebben een dergelijke verhoging met harmonische excitatie voor het eerst gedemonstreerd in een golfgeleider. Hiermee is het mogelijk geworden om deze methode in de toekomst uit te breiden en zo beduidend kortere golflengten te genereren met mengels van ionen in een capillaire ontlading.

Het tweede deel van het onderzoek dat in dit proefschrift wordt beschreven, betreft ionenversnelling en hoge harmonische generatie met vaste stoffen. Beide processen stellen hun eigen eisen aan de eigenschappen van de vaste stof en de laserpuls, bijv. de pulsintensiteit, de duur en het contrast. Bij het gebruik van laserpulsen met een hoge intensiteit en ultrahoog contrast kunnen beide processen gelijktijdig optreden omdat de twee schijnbaar verschillende processen gebaseerd zijn op dezelfde elektronendynamica. Wanneer de laserpuls het doelwit treft, wordt de vaste stof bijna onmiddellijk en volledig geïoniseerd wat resulteert in een compact en reflecterend plasma. De interactie van dit plasma met de laser leidt tot het verwarmen van een deel van de vrije plasma elektronen tot relativistische energieën. In de volgende stappen van de dynamica zijn deze elektronen verantwoordelijk voor de generatie van hoge harmonischen of versnelde ionen, wat afhangt van de eigenschappen van de laserpuls en de vaste stof.

Twee mechanismen voor harmonische generatie zijn onderdeel van het onderzoek geweest dat in dit proefschrift is beschreven om tot een verbeterd begrip en optimalisatie te komen. Het eerste mechanisme wordt beschreven met het model van de Relativistisch Oscillerende Spiegel. De hoge harmonischen worden via uiterst sterke Dopplerverschuivingen geproduceerd tijdens de reflectie van het laserlicht op de relativistische elektronen die zich uit het plasma in 
het vacuüm naar de laserpuls toe bewegen. Het tweede mechanisme, genaamd Coherente Kielzog Emissie, is gebaseerd op de laser-gedreven terugkeer van de relativistische elektronen in het plasma, waar ze collectieve en coherente oscillaties opwekken in hun kielzog. Deze plasma-oscillaties zenden straling uit in de vorm van hoge harmonischen.

De elektronendynamica die tot ionenversnelling leidt, is soortgelijk maar kan geoptimaliseerd worden door gebruik te maken van een dun folie als doelwit. Als de folie voldoende dun is, beweegt een deel van de zeer energetische elektronen zich door de folie heen en verlaat deze via het achteroppervlak waar het een compacte elektronenwolk vormt. Deze wolk produceert een groot ruimteladingsveld dat ionen vanaf het achteroppervlak van het folie tot $\mathrm{MeV}$ energieën versnelt. Dit proces wordt "Target Normal Sheath Acceleration" genoemd.

Voor nieuw experimenteel onderzoek naar ionenversnelling en hoge harmonische generatie met gebruikmaking van vaste stoffen, is een laser met hoge kwalificaties ter beschikking gesteld in een nauw samenwerkingsverband met de groep Physique à Haute Intensité. Hun laser levert pulsen met een relativistische intensiteit en een record-brekend ultrahoog pulscontrast. Speciale aandacht kregen de vervaardiging van en het onderzoek met uiterst dunne folies, dat wil zeggen vrijstaande nanofolies, om een veelbelovend maar grotendeels onverkend regime te onderzoeken. Dit regime van laser-materie interacties, genaamd het transparante regime waar de plasmadikte vergelijkbaar is met de penetratiediepte van het laserlicht (een paar nanometers), was tot dusver alleen theoretisch onderzocht. Een aantrekkelijke voorspelling is dat dit regime zou resulteren in een groter aantal versnelde ionen met een verhoogde maximumenergie. Echter, in deze voorspellingen werd een belangrijk fysisch effect verwaarloosd, namelijk de voortijdige expansie van het plasma voordat de aandrijfpuls zijn piekintensiteit bereikt.

Om de geldigheid van deze voorspellingen te testen, onderzochten we voor het eerst experimenteel de ionenversnelling in dit transparante regime met behulp van de uiterst dunne folies en de laserpulsen met hoge intensiteit en ultrahoog contrast, en we vergeleken de resultaten met data van verbeterde numerieke modellering. We onderzochten ook hoge harmonische generatie voor het eerst in het transparante regime via experimenten en numerieke modellering. Op basis van onze resultaten kan worden geconcludeerd dat het belangrijk is om rekening te houden met de kleinschalige pre-expansie van het plasma, dat veroorzaakt wordt door de ondergrond van de laserpuls met ultrahoog contrast. Bovendien, om de theoretisch interessante regimes van ionenversnelling en harmonische generatie in experimenten te bereiken is een verder verhoogd pulscontrast vereist op de tijdschaal van picoseconden. Verdere verbeteringen worden verwacht als vrijstaande nanofolies met een hogere beschadigingsdrempel kunnen worden gerealiseerd zodat de pre-expansie van de folie verminderd wordt.

We hebben ook nieuwe experimenten uitgevoerd met bulk vaste stoffen. Hiermee hebben we kunnen vaststellen dat de hoge harmonische straling die via coherente kielzog emissie geproduceerd wordt, ruimtelijk coherent is en dat de 
optische fase van de XUV emissie via de intensiteit van de aandrijvingslaser kan worden geregeld. In deze experimenten genereerden we drie foci met regelbare intensiteit op korte afstand van elkaar op het oppervlak van de vaste stof. Op deze manier realiseerden we drie XUV bronnen die onderling gesynchroniseerd zijn, zodat in het verre veld interferentiepatronen waargenomen konden worden. Met deze interferentiepatronen was het ook mogelijk om de dynamica van de plasmaelektronen tijdens de interactie met de laser te bestuderen. Dit is van groot belang om meer van de fysische details van laser-materie interacties bij hoge intensiteit te begrijpen, zoals de absorptie van licht bij relativistische intensiteit in plasma met hoge dichtheid, en de efficiënte generatie van bundels van uiterst energetische deeltjes en coherente röntgenstraling. 


\section{Contents}

Publications vii

$\begin{array}{lll}\text { Summary } & \text { ix }\end{array}$

Samenvatting xiii

1 Introduction 1

1.1 Terawatt CPA laser systems . . . . . . . . . . . . . . . 7

1.1.1 Temporal contrast enhancement . . . . . . . . 9

1.2 Thesis outline . . . . . . . . . . . . . . . 9

2 Theory of high harmonic generation with gaseous media 13

2.1 Basic laser-plasma physics . . . . . . . . . . . . . . . . . 14

2.1.1 Electromagnetic waves in a plasma . . . . . . . . 15

2.2 High harmonic generation . . . . . . . . . . . . . . . . 17

2.2.1 Microscopic description . . . . . . . . . . . . 18

2.2.2 Macroscopic description . . . . . . . . . . . . 22

2.2.3 Enhancement of HHG . . . . . . . . . . . . . . . . 23

2.3 Discussion .................... . . . . 31

3 Theory of high-order harmonic generation and ion acceleration from solids

3.1 Absorption of laser energy by plasmas . . . . . . . . . . . . . 35

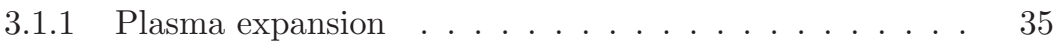

3.1.2 Collisional absorption . . . . . . . . . . . . 36

3.1.3 Collisionless absorption ............. . . 38

3.2 Ion acceleration . . . . . . . . . . . . . . . . . . 43

3.2.1 Target normal sheath acceleration . . . . . . . . . . 44

3.2.2 Influence of pulse contrast and foil thickness . . . . . . . 46

3.2.3 Ion acceleration in the transparent regime . . . . . . . . 48

3.3 High-order harmonic generation . . . . . . . . . . . . . . . 48

3.3.1 Relativistic Oscillating Mirror . . . . . . . . . . . 49

3.3.2 Coherent Wake Emission . . . . . . . . . . . . . . . . 53

3.4 Discussion ........................ 59

xvii 
4 Generating high harmonics in gas-filled capillaries $\quad 61$

4.1 Experimental setup . . . . . . . . . . . . . 62

4.1.1 Capillary and laser coupling . . . . . . . . . . . 65

4.1 .2 Diagnostics .................. 68

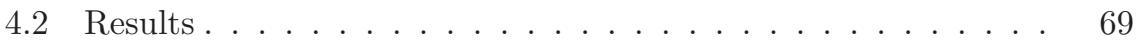

4.3 Discussion . . . . . . . . . . . . . . . 72

5 Generating high-order harmonics and fast ions from solids $\quad \mathbf{7 5}$

5.1 Foil preparation . . . . . . . . . . . . . 77

5.2 Experimental setup . . . . . . . . . . . . . . . 83

5.3 Experimental results . . . . . . . . . . . . . . 85

5.3.1 Coherent dynamics of overdense plasmas . . . . . . . . 85

5.3.2 Proton acceleration from nanofoils . . . . . . . . . 90

5.3.3 Harmonic generation from nanofoils . . . . . . . . . . . 95

5.4 Numerical modeling . . . . . . . . . . . . . . . . . . . 103

5.4.1 PIC calculations based on experiments with UHI10 . . . 104

5.4.2 PIC calculations based on experiments with UHI100 . . 108

5.4.3 PIC calculations on high-order harmonic generation . . 115

5.5 Discussion . . . . . . . . . . . . . . . . . 120

6 Conclusions and discussion

\section{Appendix}

A CPA pulse contrast $\quad \mathbf{1 2 9}$

A.1 Temporal contrast of UHI100 . . . . . . . . . . . . . . . 129

$\begin{array}{ll}\text { B Absorption coefficients } & 131\end{array}$

B.1 Classical collisional absorption . . . . . . . . . . . . 131

B.2 Normal Skin Effect . . . . . . . . . . . . . . . . . . . 133

B.3 Resonance absorption . . . . . . . . . . . . . . . . 134

B.4 Brunel absorption ................... 135

C Harmonic chirp $\quad \mathbf{1 3 6}$

C.1 Harmonic chirp due to the laser pulse envelope . . . . . . . . 136

D Target properties $\quad 138$

D.1 Maximum electron density and plasma frequency . . . . . . . . 138

E Data of proton acceleration $\quad \mathbf{1 3 9}$

E.1 Data extrapolation for 11 and $19 \mathrm{~nm}$ thick carbon foils . . . . . 139

F Evolution of electron density $\quad 141$

$\begin{array}{lr}\text { Bibliography } & 146\end{array}$

$\begin{array}{lr}\text { Acknowledgements } & 165\end{array}$ 
Ralph Smith (1858 - 1917)

\section{1 \\ Introduction}

Past exotic laser research and development has led to a general attention for high-intensity lasers and to a growing interest in their possible applications. High-intensity $\left(\gtrsim 10^{11} \mathrm{~W} / \mathrm{cm}^{2}\right)^{1}$ lasers have now found applications in important fields such as medicine, energy and environment. Examples of such applications are eye surgery via photodisruption (cutting with laser-induced plasmas) [1], laser fusion [2] and remote detection of biological aerosols in the higher atmosphere ${ }^{2}[3]$. High-intensity lasers have also found applications in fundamental research including, but not limited to, materials science, condensed matter physics, chemistry, biology and astronomy [4-12]. The main source of such developments is the wide range of nonlinear interactions between high-intensity lasers and matter. This offers novel possibilities and may lead to new applications.

Shortly after the invention of the laser by Theodore Maiman in 1960 [13] the first nonlinear optical effects were demonstrated, starting with second harmonic generation [14]. The continuous research to discover new nonlinear light-matter interactions drove the laser community to the development of lasers with ever increasing powers. Two key developments that enabled the ultra-high light intensities that are available today, were the invention of the Kerr-lens induced self-mode-locked Ti:sapphire $\left(\mathrm{Ti}: \mathrm{Al}_{2} \mathrm{O}_{3}\right)$ laser [15] and the Chirped Pulse Amplification (CPA) technique [16].

The very broad gain bandwidth (650 to $1100 \mathrm{~nm}$ ) and the high thermal conductivity of the titanium doped sapphire crystal make this material ideally suited to the generation and amplification of ultrashort pulses [17]. Commer-

\footnotetext{
${ }^{1}$ Approximate damage threshold of materials.

${ }^{2}$ TeraMobile. http://www.americanscientist.org/issues/id.990,y.2006,no.2,content.true, page.1,css.print/issue.aspx
} 
cially available Ti:sapphire laser oscillators routinely generate ultrashort pulses with a duration of 20 femtoseconds (fs) and low pulse energies of a few nanojoule $^{3}$. Attempting to amplify pulses of such an ultrashort duration to energies of several milli-joules or Joules would induce nonlinear distortion to the beam profile and damage the optics [17]. CPA avoids this problem. Before the amplification process, the ultrashort laser pulse is first stretched in time to typically several hundreds of picoseconds by introducing a, so-called, chirp by realizing a frequency-dependent optical delay with a pair of gratings or prisms. After several stages of optical amplification with a total factor of $10^{7}$ to $10^{9}$, the pulse is then recompressed to femtosecond duration. Utilizing this technique, lasers with peak powers of terawatt $\left(1 \mathrm{TW}=10^{12} \mathrm{~W} / \mathrm{cm}^{2}\right)$ to even petawatt $\left(1 \mathrm{PW}=10^{15} \mathrm{~W} / \mathrm{cm}^{2}\right)$ are currently operational ${ }^{4}$.

The use of these ultrashort, high-intensity laser pulses has opened up a number of novel research avenues in nonlinear optics, so-called extreme nonlinear optics or relativistic optics [18]. These include the generation of coherent $\mathrm{X}$-rays and attosecond pulses by high-harmonic generation in gases [19] and from solid-state targets [20], laser fusion [2], the acceleration of electrons to relativistic energies $[21,22]$, and the acceleration of protons and ions from solid targets [23].

Accordingly, many new possibilities and applications have been found as a result of these ultrashort high-intensity laser-matter interactions. This offers alternative or complementary approaches to existing applications which require large facilities such as: synchrotrons, linear particle accelerators and free electron lasers. These large facilities produce accelerated particles and (X-ray) radiation for applications in materials science, condensed matter physics, biology and medicine among others. However, due to the typical size and related costs, these facilities are rather exclusive and access to them is limited. This impedes progress in the fields of research associated with these facilities. The high potential of a high-intensity laser which can also deliver accelerated particles and X-ray radiation, is that the relatively compact laser systems would enable the research in a larger number of research groups.

For example, experimental and clinical progress has been made in ion beam cancer therapy via synchrotrons in the past ten years ${ }^{5}$. However, a comprehensive theoretical description of the physical process and the experimental clinical studies are missing $[24,25]$. This is often used as an argument against this alternative cancer treatment. Here, laser-accelerated ion beams have been identified to permit broad and systematical clinical studies with relatively compact TW lasers $^{6}$. In addition, fast ion generation via high-intensity lasers has been suggested as an alternative source for ion beam therapy $[10,26]$. The reason for

\footnotetext{
${ }^{3}$ http://www.kmlabs.com/oscillator.htm

${ }^{4}$ http://laserstars.org/biglasers/pulsed/short/index.html

${ }^{5}$ Currently, there is a discussion in the Netherlands regarding the possible building of three expensive facilities for proton beam therapy based on standard accelerators. http://www.protonconferentie.nl/

${ }^{6} \mathrm{An}$ example is the cancer research at the Forschungszentrum Dresden Rossendorf: http://www.fzd.de/db/Cms?pNid=265
} 
this is that the laser-driven ion beams offer a combination of attractive physical properties, including, high brightness, low transverse divergence, a high laminarity, multi-MeV energies and an ultrashort duration. These properties make these ion beams also very promising for other applications in engineering and medicine with examples including: high-brightness injectors for accelerators, radiography and isotope production for PET scanners [6,27]. The application of such ion beams in border security in helping the detection of explosives, narcotics and other dangerous substances has also been suggested [12]. Furthermore, via proton imaging the laser-accelerated proton beams can provide increased contrast and spatio-temporal resolution as a plasma inspection tool, as shown via probing ultrafast laser-driven plasma dynamics [28].

However, before laser accelerated ions can be applied as described, significant advances have to be made with respect to the maximum ion energy, the energy spread, the ion current and the stability. This requires further development in high-intensity laser technology and fundamental research in the field of laser-based ion acceleration [29].

A second example of the high potential of high-intensity lasers is high harmonic generation, which is currently the only way to generate extreme UV (XUV) and X-ray radiation in the form of pulses with a duration in the attosecond regime $[30,31]$. High harmonic generation means that a large number of frequencies which are multiples of the original frequency of the incident drive laser, are generated. By proper filtering of the spectrum, attosecond pulses can then be obtained [32]. In addition to this unique property, the harmonic source enables complementary research to the applications of large facilities such as the XFEL (X-ray Free Electron Laser) in Hamburg, Germany.

The generation of ultrashort highly-directional coherent X-rays by high harmonic generation can become a key approach to enabling imaging on the atomic scale and to probe ultrashort molecular dynamics on the femtosecond time scale and, electron dynamics on the attosecond time scale [33-36], for example, via soft X-ray microscopy or lensless diffractive imaging [37,38]. Harmonic XUV and X-rays can also be used to probe hot dense plasmas that are formed, for example, during the process of laser-driven ion acceleration [39]. Another application would be X-ray interference lithography that requires coherent radiation. With X-ray interference lithography, still based on synchrotrons, nanostructured patterns were obtained with a resolution as small as $11 \mathrm{~nm}[40,41]^{7}$. An additional advantage of X-rays generated via high harmonic generation is that this radiation possesses a higher degree of spatial coherence and spectral coherence than X-rays generated from synchrotrons and undulators [42]. If a higher coherence is required from X-FEL facilities, injection seeding is a good possibility where high harmonic generation is a very promising approach [43]. It can also be expected that the harmonic radiation will lead to novel research and applications in the field of nonlinear optics in the regime of XUV and $\mathrm{X}$-rays, due to the high peak intensity that would be achievable because of

\footnotetext{
${ }^{7}$ http://lmn.web.psi.ch/xil/index.html
} 
the ultrashort duration and the possibility of focusing such short wavelength radiation, in principle, to an ultra-small (sub-nanometer) spot [44].

A disadvantage is that the process of high harmonic generation currently offers a relatively low conversion efficiency. Improving the output, with respect to the efficiency and shortest attainable wavelengths, will unmistakably simplify present measurement techniques, broaden current research and significantly expand the scope of applications in the future [32].

A third example of the high potential of high-intensity lasers, is the laser-based acceleration of electrons, such as via the laser wakefield in a plasma [21]. This mechanism can accelerate electron bunches to $\mathrm{GeV}$ energies of femtosecond duration in plasmas of only a few centimeters long. Such electron bunches would enable the generation of femtosecond X-ray pulses in small-sized undulators or, it could be used as an injector for existing free electron lasers [45]. In recent years, a demonstration experiment for a laser wakefield accelerator has been assembled and is currently being tested in our group [46, 47] (the Laser Physics and Nonlinear Optics (LPNO) group at the University of Twente) ${ }^{8}$. The investigated scheme of laser wakefield acceleration is unique in that it may allow the first acceleration of an externally injected electron bunch, and the first acceleration after injection in front of the laser pulse. The advantage to be gained is that a more stable and more controlled acceleration of the electron bunch might be obtained compared to other schemes of laser wakefield acceleration [22]. The experiment makes use of a home-built CPA terawatt laser. The output power is at the moment $12 \mathrm{TW}$ (UTTW12), but will be upgraded to $30 \mathrm{TW}$ in the future. With such peak powers, the laser can also be considered for high harmonic generation and ion acceleration.

In this thesis, results will be presented from research that has been performed in two of the previously named fields of high-intensity laser-matter interactions, ion acceleration and high harmonic generation. It shows that these two processes are closely related to each other and, in fact, the generation of fast ions and high harmonics can occur simultaneously when irradiating a solid target with high-intensity laser pulses. In addition, high harmonics can also be generated by focusing high-intensity laser pulses into gaseous media, however, the much lower density of a gas necessitates a completely different physical mechanism. An essential property of harmonic generation in gases is that the radiative electrons are bound which limits the highest harmonic frequency to a maximum value (the shortest wavelength), the so-called cut-off frequency. Harmonic generation with solids is based on free electrons and has no principle limitation on the maximum frequency besides limits in the applied laser intensity. In this thesis, both types of media, gases and solids, have been employed to generate high harmonics. In order to distinguish between the two harmonic processes throughout this thesis (and that is often found in litera-

\footnotetext{
${ }^{8} \mathrm{LPNO}$ is a member of the $\mathrm{MESA}^{+}$Institute for Nanotechnology
} 
ture), generation in gaseous media will be termed high harmonic generation (HHG), and generation from solids will be termed high-order harmonic generation (HOHG).

To fully enable the described applications of high-intensity lasers, the processes of ion acceleration and harmonic generation require an enhancement of the conversion efficiency of the flux and maximum energy of the accelerated ions and the harmonic radiation with respect to the incident laser energy. The scope of this thesis is to investigate methods towards this goal, via an improved understanding of the underlying physics.

In view of this, part of the work described here comprises the design, construction and test of a standard setup and suitable diagnostics for HHG (in gaseous media) using the TW laser at LPNO. This prepares for future exploration of several methods to enhance the efficiency of HHG. It also prepares for nonlinear optical experiments where XUV radiation is employed as the fundamental input radiation. Custom made XUV mirrors required for such experiments are readily available due to a collaboration with FOM Rijnhuizen [48]. The current HHG setup in LPNO uses a capillary waveguide for the generation of high harmonics in gases, plasmas or in gas mixtures because such a configuration promises a relatively high output and highly coherent (spatially and temporally) harmonic beams $[19,42,49,50]$. The capillary waveguide also may offer the opportunity to implement additional enhancement methods, for example, quasi-phase-matching via multi-mode beating [51]. Our experiments on an enhancement technique called Harmonic Excitation as presented in this thesis show promising results for future experiments in enhancing the output, for example with ions from a capillary discharge.

The other part of the research described in this thesis concerns HOHG and ion acceleration from solid targets. Special attention was given to the preparation of freestanding nanofoils and the interaction of such extremely thin foils with high-intensity laser pulses. This regime of laser-matter interaction had only been theoretically investigated to this point [52]. An attractive prediction is that the interaction of high-intensity lasers with extremely thin foils in the so-called transparent regime, i.e. with thicknesses in the order of the penetration depth of the laser light through dense plasma (a few nanometers), would result in an enhanced maximum energy and number of accelerated ions. This prediction is, however, based on numerical calculations that neglected to take into account the important physical effects of the early expansion of the plasma before the drive pulse reaches its peak intensity.

For HOHG in the transparent regime, the situation is quite comparable because the underlying interaction process during the laser pulse which is responsible for the absorption of laser energy and the subsequent heating of the plasma, is the same as with ion acceleration. As a consequence, it could be that, in analogy with predictions for ion acceleration, extremely thin foils also enhance the HOHG output. Based on these considerations and in order to gain more insight in the physical details of both HOHG and ion acceleration, 
it is important to provide experimental data from this regime. For this, it is important to identify the actual requirements on the laser and the target for optimum output.

The goal of this part of our research is to experimentally study this transparent regime for the first time. It turns out that such experiments demand extra advanced specifications for the drive laser radiation. This includes, in particular, what is called a low pedestal or a high pulse contrast (strongly reduced background radiation before the main pulse arrives). The TW laser at LPNO is optimized for a different goal (laser wakefield acceleration) where background radiation is much less relevant. To enable experiments in the transparent regime, a collaboration has been established with the group Physique à Haute Intensité (PHI) at CEA Saclay in France, where their low-pedestal TW laser was employed to irradiate nanofoils for the generation of high-order harmonics and fast ions. That laser system reaches the requirement for a low background with additional components in the laser system, such as, so-called, plasma mirrors. PHI has at her disposal a $10 \mathrm{TW}$ laser (UHI 10), which has recently been upgraded to $100 \mathrm{TW}$ (UHI 100). Both configurations of this laser at PHI were used for our experiments. Additional insight was acquired by comparing the experimental data with numerical calculations. In order to interpret our experimental data, we tested and used an available numerical model, based on the, so-called, particle-in-cell (PIC) code.

There are several other open questions concerning HOHG. For example, although HOHG from solid targets (overdense plasmas) results in collimated $\mathrm{X}$-rays beams, this property alone does not guarantee spatial coherence of the radiation [53] and an experimental verification of the coherence had not yet been executed. Another question is whether the theoretical description for a particular scheme of HOHG, called coherent wake emission, which is derived using certain assumptions in numerical modeling, is in all respects correct. For example, it predicts that the high-order harmonics have a certain intensity-dependent relationship with the spectral phase that affects the spatial coherence of the harmonic beam. We performed experiments that clarify these questions for the first time by irradiating a bulk solid target with three high-intensity spots and record the interference pattern in the far-field that is the result of the mutual coherence of the three sources.

Both laser systems at LPNO and PHI are based on CPA. Although this technique is currently the most suitable method to amplify ultrashort laser pulses, there are some limitations in the final pulse duration. These include the unavoidable presence of background radiation from amplified spontaneous emission (ASE) on the nanosecond time scale or, pedestals (wings) on the picosecond time scale or, that weak pulses (pre-pulses) are emitted before the main pulse. As a consequence, the specific limitations in the parameters of the laser, i.e., to what degree such background is reduced, determines which types of experiments the laser is suitable for. To illustrate where these limitations come from, we will briefly describe the basic components and properties of CPA lasers in 
more detail in the next section. We will also describe the extra measures that have been taken at PHI to improve the temporal contrast of the laser pulse (defined as the ratio between the main pulse intensity and the intensity of the pedestal, pre-pulses and ASE) in order to expand the experimental possibilities of such CPA lasers. At the end of the section, a table is given with an overview of the output parameters of the mentioned laser systems used in our experiments.

\subsection{Terawatt CPA laser systems}

The basic principle of chirped pulse amplification, which is schematically shown in figure 1.1, is to increase the energy of an ultrashort pulse generated by an oscillator, while avoiding very high peak powers in the amplification process itself [17]. This is achieved by, first imposing a chirp on the ultrashort pulse with a dispersive element so that the pulse duration becomes stretched before amplification. Then the pulses pass one or more optical amplifiers and, finally, a second dispersive element which compresses the pulse in time so that it is close to its original duration.

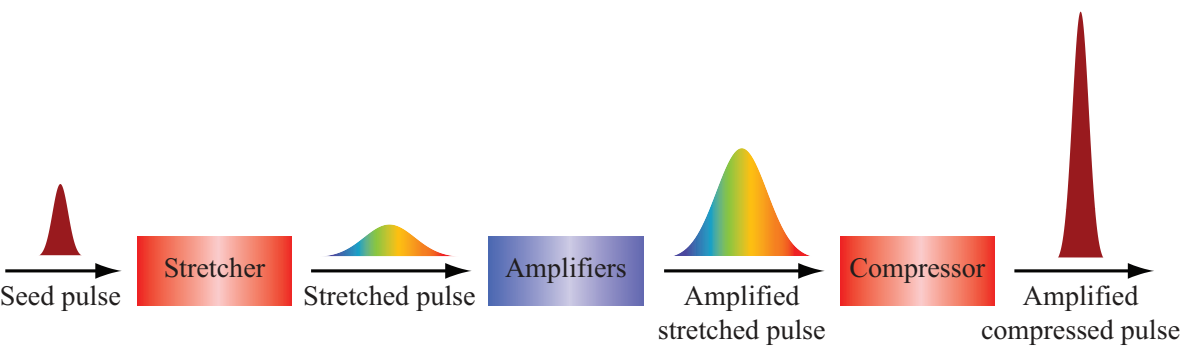

Figure 1.1: Chirped pulse amplification: an ultrashort laser pulse of low energy is stretched in time, after which it is amplified and subsequently compressed again, in order to obtain an ultrashort pulse of high energy while preventing damage during amplification. The colors in the pulse symbolize the relative timing of the spectral components contained in the pulse.

In both laser systems employed at LPNO and PHI, the oscillators are broadbandwidth Kerr lens mode-locked Ti:sapphire lasers, operated at a central wavelength of about $800 \mathrm{~nm}$, with a spectral bandwidth of the order of $50 \mathrm{~nm}$, enabling pulse durations of typically several tens of fs with pulse energies of a few nJ.

The stretcher and compressor make use of a pair of gratings that temporally disperse the broadband pulse. The stretchers in both laser systems are aberration-free Öffner stretchers [17]. In principle, a compressor with its dispersion perfectly matched to that of the stretcher can recompress the pulse to its 
original ultrashort duration. However, the pulse acquires additional materialdependent dispersion by passing through a number of optical elements in the amplification stages. By adjusting the grating angles and the separation distance, second- and third-order dispersion can be compensated for, but residual higher-order dispersion remains. Therefore, the corresponding pulses are not precisely Fourier-limited and will show unwanted pedestals (wings) on the pulse. This is the first limitation of CPA with respect to the final pulse duration. This problem can be compensated for to some extent with advanced techniques such as an acousto-optic programmable dispersive filter (Dazzler) ${ }^{9}$.

Other limiting effects on the pulse duration come from the amplification process. The amplification involves a high-gain preamplifier and one or more power-amplifiers. The pre-amplifier is responsible for most of the gain with a factor of $10^{6}$ to $10^{7}$, while a power amplifier provides a much smaller gain factor, in the order of 10 to 1000.

There are two basic types of preamplifiers: regenerative and multi-pass. Power-amplifiers are in general multi-pass systems. A regenerative amplifier (Regen) is basically an optically stable resonator containing a high-gain laser amplifier. The pulse is electro-optically switched into the resonator and switched out again after amplification during, typically, 10 to 20 roundtrips. The advantage of a regenerative amplifier is its high amplification factor and that it provides a stable output beam profile (approximately a Gaussian beam with a high pointing stability), because the resonator (and possibly filters that remove light in higher order modes) essentially filters the transverse beam profile with every roundtrip through the gain medium. A disadvantage of a Regen is the formation of pre- and post-pulses, caused by a small and undesired reflection of the amplified pulse at each roundtrip into the outcoupling beam path, due to a finite extinction ratio of the polarizer that is part of the switching optics. A second disadvantage of a Regen is the build up of amplified spontaneous emission (ASE), which can deplete some of the gain in the following amplifiers before it can be extracted by the short pulse. This can result in a significant noise level, appearing as a broadband background pulse with nanosecond duration. In addition, when amplifying chirped pulses, where usually the red part of the spectrum precedes the blue part, the spectrum of the pulse becomes red-shifted. This occurs because the leading (red) edge of the pulse partially depletes the inversion for the trailing (blue) edge. Another undesired effect during amplification is spectral narrowing of the light, which is caused by the curvature of the gain spectrum that provides less amplification to the spectral wings of the pulse, and thereby leads to an increased pulse duration.

The temporal contrast of the laser pulse is to some extent improved by introducing an additional optical switch (a so-called pulse slicer) into the amplifier chain after the output switch of the Regen. The contrast achieved thereby is high, in the order of $10^{6}$, which is sufficient for laser wakefield acceleration and HHG (in gases). However, the same contrast can be detrimental when focusing the amplified ultrashort pulses to extremely high intensities on solid state tar-

\footnotetext{
${ }^{9}$ http://www.fastlite.com
} 
gets, such as required for HOHG and ion acceleration. Then, even a seemingly weak background can dramatically influence the experimental results through strong ionization of the target and an undesired expansion of the generated plasma before the main pulse arrives. It can be expected that such effects, related to an insufficiently high contrast, would play a major role, especially with extremely thin targets such as foils with thicknesses in the range of a few nanometers.

\subsubsection{Temporal contrast enhancement}

To prevent such effects, extra measures have to be taken in order to improve the temporal contrast of the laser pulses. At the $100 \mathrm{TW}$ laser at PHI (UHI 100), an additional pre-amplifier is installed with a nonlinear filter (a so-called saturable absorber) to suppress ASE. In this way, the intrinsic ASE contrast is improved by more than 3 orders of magnitude. Also two acousto-optic, programmable dispersive filters (Dazzlers) are employed to reduce the power in undesired pedestals of the pulse by compensating higher-order dispersion.

Additionally, for HOHG and ion acceleration from solids, it is essential to further increase the contrast, especially with respect to the pre-pulses. At PHI this is done with a so-called double plasma mirror (DPM) [54-57], in both the UHI 10 and UHI 100 configurations. This DPM consists of two subsequent dielectric plates with an anti-reflection coating for $800 \mathrm{~nm}$. The beam cross section of the laser output on the plates is adjusted such that the fluence of the ASE and pre-pulses remains just below the damage threshold, such that both the ASE and pre-pulses are transmitted and do not reach the target. However, in the rising edge of the main pulse, where the fluence grows to much higher values, the surface of the plates is ionized and forms a plasma that reflects most of the main pulse fluence towards the target. In this manner, the temporal contrast is enhanced by 4 orders of magnitude, while preserving the laser beam profile $[57,58]$. The efficiency of the DPM is about $50 \%$ and the resulting temporal pulse profile of the UHI 10 laser has a contrast of about $10^{10}$ [57]. The final temporal pulse profile of the UHI 100 is shown in appendix A, and here the efficiency of the DPM is about $70 \%$.

For an overview and later reference, the parameters of the lasers that have been used in our experiments, are summarized in table 1.1. For the HHG experiments in waveguiding capillaries at LPNO, only a small fraction of the TW laser output was required. This was tapped-off with an adjustable pulse energy and sent through a separate compressor (UTTW1).

\subsection{Thesis outline}

This thesis mainly reports about the experimental issues and results. A comparison with theory is provided, however, this requires numerical modeling, due to the high nonlinearity and resulting complexity of the investigated processes. 


\begin{tabular}{lrrrr}
\hline & UTTW1 & UTTW12 & UHI 10 & UHI 100 \\
\hline Energy (J) & $<0.025$ & 0.48 & 0.60 & 2.5 \\
Duration (fs) & 45 & 40 & 60 & 25 \\
ASE contrast & $\approx 10^{4}$ & $\approx 10^{4}$ & $10^{6}$ & $5 \cdot 10^{9}$ \\
Pre-pulse contrast & $\approx 10^{4}$ & $\approx 10^{4}$ & $10^{5}$ & $5 \cdot 10^{6}$ \\
Energy after DPM (J) & - & - & 0.30 & 1.7 \\
Contrast after DPM & - & - & $10^{10}$ & $10^{13}$ \\
\hline
\end{tabular}

Table 1.1: Output parameters of the CPA lasers at LPNO and PHI. The TW laser at LPNO has a split off with adjustable energy and uses a separate compressor in air (UTTW1).

In order to enable a basic discussion of the involved physical processes, the first chapters give a basic introduction into the theory of the essential physical mechanisms that are involved. Because the interaction of high-intensity lasers with gases or solids generally leads to the formation of plasmas, chapter 2 begins with a basic description of some of the important properties of plasma and the interaction of light with plasma. The remainder of that chapter is devoted to a theoretical description of high harmonic generation (HHG) in gaseous media and possible methods to improve the efficiency and increase the maximum harmonic frequency.

In chapter 3, theoretical approaches to describe high-order harmonic generation $(\mathrm{HOHG})$ and ion acceleration from solid targets will be presented. The description includes the basic interactions responsible for the absorption of the laser energy by the plasma. This depends critically on the plasma density, the profile of the plasma and on the laser pulse parameters. For this reason, the chapter starts with short descriptions of several absorption mechanisms which are relevant to the conversion of laser energy into highly energetic electrons that, in the subsequent dynamics, are responsible for the generation of high-order harmonics or the acceleration of ions.

In the intensity regime attainable with the lasers at PHI, the mechanism for ion acceleration is the so-called Target Normal Sheath Acceleration (TNSA). The basic physical description of this process will be recalled in the second section of chapter 3 . Then a description of the acceleration process will be given, including a description for the process when using extremely thin foils. When the foil thickness is in the order of the penetration depth of the laser, the plasma becomes partly transparent. The prediction is that in this transparent regime, the number and maximum energy of the accelerated ions will be increased.

The third section of chapter 3 presents a theoretical description of two distinct processes of high-order harmonic generation from solids that have currently been distinguished from each other based on their different signatures, namely, HOHG via the mechanism of the so-called Relativistic Oscillating Mirror (ROM) and HOHG via Coherent Wake Emission (CWE). 
Chapter 4 describes our experimental setup constructed for HHG at LPNO in detail, some advantages of the setup and problems that had to be solved. We present first test measurements and corresponding results which includes an enhancement technique, which we will refer to as Harmonic Excitation (HEx). This technique had previously been demonstrated by Takahashi et al. with freely propagating beams [59]. We demonstrate for the first time the working of HEx in a guided-wave geometry, using a capillary filled with a gas mixture.

In the first section of chapter 5 , a detailed description of the technique that we developed to prepare samples of freestanding, extremely thin foils, with thicknesses of only a few nanometers will be given. This technique enabled the experimental investigations of ion acceleration and HOHG into parameter regimes that have been unexplored in the past. The interaction chamber and setup at PHI used for these experiments will be described in the second section of chapter 5. The results that were obtained with this setup are described in the third section, followed by a section on numerical modeling in order to interpret our experimental results. The chapter ends with a discussion of the results.

In the last chapter 6 of this thesis, our results are summarized and discussed. 
$\bigoplus$ 


\section{2 \\ Theory of high harmonic generation with gaseous media}

Part of this thesis reports on the experimental implementation of a high harmonic generation (HHG) setup at LPNO with the goal of producing intense coherent XUV pulses to perform nonlinear optics in the XUV and soft X-ray regime. One motivation for this is that, through a collaboration with FOM Rijnhuizen, we have access to XUV optics required for such experiments, in particular, focusing and filtering optics made from multi-layered MoSi mirrors. In addition, our HHG project supports a second program that aims to develop other XUV optical elements, e.g. Fresnel-Bragg optics, for which a compact, high-brightness coherent XUV source is highly desirable to characterize their functionality.

The objectives of the first part of the research described in this thesis are: the realization of the setup, the performance of test measurements and the investigation of methods to enhance the harmonic yield and increase the maximum harmonic order. For these goals, we eventually aim to utilize capillary discharges to generate high harmonics from ions in plasmas. In our group, knowledge about capillary discharges is readily available thanks to previous research projects.

In order to provide a better comprehension of the experimental considerations and results on the HHG setup, discussed in chapter 4, this chapter describes the basic theory of HHG in gaseous media. This thesis also reports on experimental results from high-order harmonic generation (HOHG) and ion acceleration from plasmas formed from solid targets. Because in all these cases the properties of plasmas play a central role, we first recall in section 2.1 the essential theory of plasmas and its interaction with laser light. We also provide 
definitions of several physical variables that will be used throughout this thesis.

\subsection{Basic laser-plasma physics}

Plasma, or the "fourth state of matter" as it is sometimes referred to, is a gas-like but ionized state of matter with approximately equal numbers of positively charged ions and negatively charged electrons (and sometimes neutral particles) which exhibits collective behavior [60]. Collective behavior means that the motion of charged particles does not depend only on local conditions but also on the state of the plasma in remote regions. This is because the motion of charged particles induces strong electric and magnetic fields that affect the motion of other charged particles far away in the plasma. These long-range electromagnetic forces are much larger than the forces due to ordinary short-range interactions like collisions, such that the latter can often be neglected. This approximation is also known as "collisionless" plasma (see also section 3.1.3).

Two important properties of plasmas that are due to the collective behavior are Debye screening and plasma oscillations. Debye screening means that the electric potential of an individual, charged particle or of externally applied field, e.g. an incident laser field, is screened by nearby and mobile charges within a distance $\lambda_{D}$ called the Debye length [61]. In other words, the plasma tends to neutralize any disturbance of the potential. The Debye length is described by

$$
\lambda_{D}=\sqrt{\frac{\epsilon_{0} k_{B} T_{e}}{n_{e} e^{2}}} .
$$

Here $n_{e}$ is the electron density and $k_{B} T_{e}$ is the electron temperature, which is related to the thermal velocity $v_{e}$ by

$$
v_{e}=\sqrt{k_{B} T_{e} / m}
$$

assuming a Maxwellian velocity distribution [61]. $m$ is the electron mass. This Debye screening is only effective if there are enough particles $(\gg 1)$ within the so-called Debye sphere, the number of which is given by

$$
N_{D}=\frac{4 \pi}{3} \lambda_{D}^{3} n_{e}
$$

In our experiments on HHG, using for example singly-ionized xenon gas at a Xe density in the range of $2.5 \cdot 10^{17} \mathrm{~cm}^{-3}(10 \mathrm{mbar})$ and an electron temperature of $10 \mathrm{eV}$, the plasma has a Debye length of almost a micrometer, containing more than a thousand electrons in the Debye sphere. In comparison, for solid-density plasmas $\left(10^{23} \mathrm{~cm}^{-3}\right)$ with a temperature of $1 \mathrm{keV}$, as described in chapter 3, the Debye length is less than 1 nanometer, and contains only approximately a hundred particles.

The second property of a plasma, to show plasma oscillations, can be seen as 
follows. If electrons are displaced from a uniform background of ions, electric fields will build up in a direction that restores the neutrality of the plasma again by pulling electrons back to their original positions [60]. Due to this restoring force and the inertia of the electrons, the plasma electrons start to oscillate at a characteristic frequency, known as the plasma frequency. The plasma frequency is given by $[60,61]$

$$
\omega_{p}=\sqrt{\frac{n_{e} e^{2}}{\epsilon_{0} m}} .
$$

Because of the relatively low mass of the electrons compared to the ions, these plasma oscillations are so fast that the ions may be considered immobile. In the ideal situation of an infinite plasma with small disturbances, the electrons behave like mutually independent oscillators. However, this last approximation no longer holds when a sudden charge imbalance in a finite system causes the motion of charged particles to screen and neutralize the disturbance. The plasma oscillations then propagate and a longitudinal plasma wave, which can propagate far beyond the region of the initial disturbance, is initiated.

To summarize, the Debye length indicates the separation between short and long range interactions in a plasma [61]. Collisions between charged particles mainly occur within the Debye length and collective motions, such as plasma oscillations, occur beyond this distance.

\subsubsection{Electromagnetic waves in a plasma}

The property of plasmas to react collectively to a perturbation with a restoring oscillation at the plasma frequency has an important implication for electromagnetic waves interacting with plasmas: radiation with a frequency larger than the plasma frequency can propagate in a plasma. This situation is called that of an underdense plasma. Qualitatively, this can be seen from the wave equation in plasmas [61]:

$$
\frac{\partial^{2}}{\partial t^{2}} \mathbf{E}(\mathbf{r}, t)=\left(\omega_{p}^{2}+c^{2} \nabla^{2}\right) \mathbf{E}(\mathbf{r}, t),
$$

which results in the dispersion relation for plane waves

$$
\omega_{0}^{2}=\omega_{p}^{2}+k^{2} c^{2},
$$

where $\omega_{0}$ is the light frequency and $k$ is the wave number. This implies that the phase velocity of light in plasma is greater than the phase velocity of light in vacuum, $c,[60]$

$$
v_{\phi}=\frac{\omega_{0}}{k}=\sqrt{\frac{\omega_{p}^{2}}{k^{2}}+c^{2}}=\frac{c}{\widetilde{n}}
$$

or, alternatively, that the index of refraction, $\tilde{n}$, is smaller than 1 :

$$
\widetilde{n}=\sqrt{1-\frac{\omega_{p}^{2}}{\omega_{0}^{2}}} .
$$


The group velocity $\partial \omega / \partial k$, derived from equation 2.6 as

$$
v_{g}=c \sqrt{1-\frac{\omega_{p}^{2}}{\omega_{0}^{2}}}
$$

remains below $c$.

As becomes apparent from equation 2.8, the index of refraction becomes imaginary for $\omega_{p}>\omega_{0}$, for example, when increasing the plasma frequency above the light frequency via an increase in the electron density (according to equation 2.4). This situation is referred to as an overdense plasma. In this situation, the wave is reflected by the plasma while, in the forward direction, the amplitude decays exponentially over a distance $l_{s}$, known as the collisionless skin depth or penetration depth [62]:

$$
l_{s}=\frac{c}{\omega_{p}}\left(1-\frac{\omega_{0}^{2}}{\omega_{p}^{2}} \cos ^{2} \theta\right)^{-1 / 2},
$$

with $\theta$ being the angle of incidence. In highly overdense plasma the penetration depth becomes $l_{s} \approx c / \omega_{p}$. The transition between reflection and transmission is determined by the, so-called, critical density $n_{c}$, where $\omega_{0}$ equals $\omega_{p}$. This resonance condition leads to an efficient coupling between the electromagnetic wave and the plasma as will be shown in section 3.1. For a laser wavelength of $800 \mathrm{~nm}$, which is typical for experiments with intense ultrashort light pulses, the critical density

$$
n_{c}=\frac{\epsilon_{0} m \omega_{0}^{2}}{e^{2}}
$$

is $1.7 \cdot 10^{21} \mathrm{~cm}^{-3}$. In comparison with the typical densities obtained by ionizing solid-state materials, fully ionizing carbon (with a density of $2 \mathrm{~g} / \mathrm{cm}^{3}$ ) yields an electron density of about 340 times the critical density. Thus, for this wavelength, fully ionized carbon is a highly overdense plasma and from equation 2.10 one can derive that the infrared light penetrates only about $7 \mathrm{~nm}$. Note, that this is twenty times less than for non-ionized carbon [63].

The above-mentioned equations require a modification for sufficiently high laser intensities, namely, when the electrons oscillate with velocities near the speed of light. Due to the relativistic mass of the electrons, the plasma frequency (equation 2.4) changes to $\omega_{p} / \sqrt{\gamma}$, where $\gamma$ is the relativistic factor or Lorentz factor as given below.

The oscillatory motion of electrons in an electromagnetic field becomes relativistic for a laser intensity that corresponds to the condition when the normalized amplitude parameter, $a_{0}$, equals 1 [64]:

$$
a_{0}=\frac{p_{o s}}{m c}=\frac{\gamma v_{o s}}{c}=\frac{e E_{0}}{m c \omega_{0}}=\sqrt{\frac{I_{0} \lambda_{0 \mu}^{2}}{1.37 \cdot 10^{18}}} .
$$

Here, $p_{o s}\left(v_{o s}\right)$ is the transverse quiver momentum (velocity) of an electron in a laser field with an electric field amplitude $E_{0} . I_{0}$ is the laser intensity in units 
of $\mathrm{W} / \mathrm{cm}^{2}$ and $\lambda_{0 \mu}$ is the laser wavelength in microns. $\gamma$ is the Lorentz factor, $\gamma=\sqrt{1+p^{2} / m^{2} c^{2}}[62]$.

Now that the basic theory on plasmas and its interaction with light has been described, a theoretical description of HHG in gases and underdense plasmas will be presented in the next section.

\subsection{High harmonic generation}

High harmonic generation (HHG) is an extreme nonlinear optical process of frequency up-conversion. In the past extensive theoretical and experimental research has been performed on the generation of high harmonics in gas-like media (atoms, molecules or ions) and its characterization. In this chapter, some aspects of HHG from atoms and ions related to our experiments (described in chapter 4) will be discussed. For more details, the reader is referred to reviews as in $[19,50,65-70]$.

Firstly, the basic properties of HHG are summarized. Understanding these properties involves a description of the process on the microscopic and also the macroscopic level. Most of the microscopic properties can be explained by the semi-classical three-step model, also referred to as the recollision model or simple man's theory [71,72], as will be recalled here. A more accurate description of the response of a single atom requires a quantum mechanical approach [73,74], from which we will only recall its predictions regarding the phase of the generated harmonics, because this is of importance for understanding the coherence properties of HHG at the macroscopic level, such as observed in interference experiments. The macroscopic effects of phase-matching and absorption are briefly described in subsection 2.2 .2 , with an emphasis on propagation in a gas-filled hollow waveguide as this is the configuration we use in our experiments. As a method of increasing the efficiency of HHG for a potential use in applications, the final subsection is devoted to the enhancement of HHG with respect to both the harmonic yield and the extension of the cut-off frequency.

High harmonics are generated by focusing a high-intensity linearly polarized laser pulse into a gaseous media. The strongly nonlinear interaction between the laser electric field and the atom results in ultrashort and highly-directional coherent radiation. This radiation contains a large number of harmonics of the drive laser frequency and can extend into the regime of the extreme ultraviolet (XUV) and soft X-rays. To date, the highest photon energy produced with HHG in gases is $3.5 \mathrm{keV}$ ( $0.35 \mathrm{~nm}$ wavelength), however, only at the level of a few photons per pulse [75].

A typical property of $\mathrm{HHG}$ is that the radiation is emitted in a train of chirped attosecond pulses in the direction of the drive laser propagation. Another feature is the characteristic shape of the output spectrum, which is schematically shown in figure 2.1. The spectrum contains only odd harmon- 


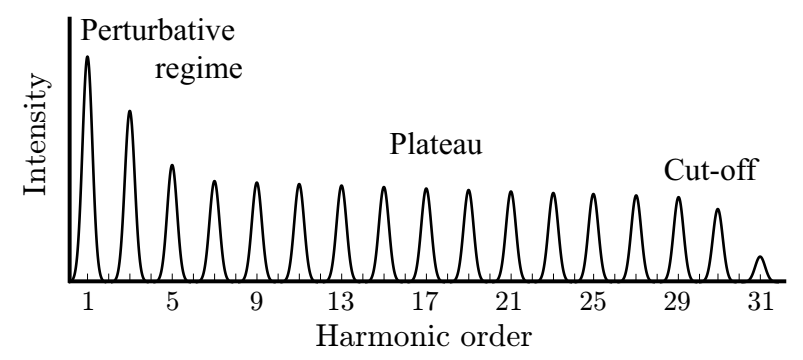

Figure 2.1: Characteristic spectrum of HHG. Only the output from the first few harmonics can be understood in terms of the perturbative theory of nonlinear optics. Extreme nonlinear optics results in high-order harmonics in the plateau region up to the cut-off region.

ics and shows, what is called, a plateau, where the harmonic output remains almost constant with increasing harmonic order. Beyond the plateau one observes the so-called cut-off, where the output drops drastically. The conversion efficiency of HHG depends on: the type of medium used, the harmonic order, the intensity and pulse duration of the driving laser, and the geometry used for the generation (for example, a gas jet or a gas-filled hollow waveguide). Typically the efficiency is rather low. The best efficiencies of up to $10^{-5}$ are obtained at relatively low orders $(q \approx 20)$. The efficiency drops to less than $10^{-7}$ for high orders $(q>50)$ down to $10^{-11}$ for cut-off harmonics beyond the $300^{\text {th }}$ order [76]. The highest efficiencies are generally obtained with short pulse durations to suppress ionization and in hollow waveguides to extend the interaction length as compared with gas jets [19].

In order to give insight into these properties, a description of the process on the microscopic level will be described next.

\subsubsection{Microscopic description}

The semi-classical three-step model, summarized in figure 2.2a provides a physical picture on how a single atom generates high harmonic radiation [71,72].

1) In the first step, the applied laser field distorts the atomic Coulomb potential (dashed curve) with a superimposed potential slope (solid curve). As a result, through tunnel-ionization a part of the electron wave packet is ionized into the continuum with a low kinetic energy.

2) Subsequently, the ionized part of the electron wave packet propagates freely in the continuum under the influence of the laser field. Here, the electron motion can be understood easiest when describing it classically as a particle that is accelerated and decelerated by the electric field of the laser. The electron trajectory is very sensitive to when ionization occurs with respect to the phase of the laser field, as indicated in figure $2.2 \mathrm{~b}$. If ionized at the proper instant 
(phase), the electron is accelerated back towards its parent ion, acquiring a certain amount of kinetic energy.

3) The last step involves possible recombination of the electron. When the accelerated wave packet returns to the parent ion and interferes with the part of the wave packet that was left behind in the original orbital, the resulting rapidly-oscillating dipole emits high-frequency radiation with an energy that equals the ionization potential plus the kinetic energy that the electron has acquired. In a classical description one can consider this last step as a strong deceleration, in the vicinity of the atomic core, of the charged electron particle that consequently emits radiation.

The energy that the electron acquires during the second step can be estimated from the electron's excursion trajectory as follows. Each trajectory is defined by the instant of ionization and yields a certain instant of recombination, illustrated in figure $2.2 \mathrm{~b}$. As shown in the graph, only electrons being ionized with a phase between $0^{\circ}$ and $90^{\circ}$ have a chance to recombine and emit high harmonic radiation. Furthermore, a distinction can be made between long and short trajectories and the cut-off trajectory. A harmonic frequency in the plateau of the spectrum can result from both short and long trajectories (indicated in figure $2.2 \mathrm{~b}$ ) that yield an equal electron kinetic energy. The light waves generated by these two dominant trajectories generally interfere non-constructively with each other [50]. In between these long and short trajectories there is a single trajectory that corresponds to the cut-off. The integration of motion along the electron trajectories can be used to determine the electron's kinetic energy as a function of the instant of ionization. The calculation shows that when the electron is ionized at a phase of $18^{\circ}$ after the peak of the electric field of the laser, the electron acquires maximum kinetic energy before it recombines. Accordingly, electrons being ionized between $0^{\circ}$ and $18^{\circ}$ (or $180^{\circ}$ and $198^{\circ}$ ) correspond to the long trajectories. Ionization occuring between $18^{\circ}$ and $90^{\circ}$ (or $198^{\circ}$ and $270^{\circ}$ ) lead to short propagation times. Conversely, electrons that are ionized before the peaks of the electric field never return.

When performing the integration of motion, one finds the maximum kinetic energy that the electron acquires. Upon recombination a further atomic ionization energy, $I_{p}$, is released. These combined energies give the maximum total photon energy available for HHG

$$
\hbar \omega_{\max }=I_{p}+W_{k i n, \max }=I_{p}+3.17 U_{p} .
$$

This expression is also known as the cut-off rule for HHG. Here, $U_{p}$ is named the ponderomotive potential, which is the time-averaged kinetic quiver energy associated with the oscillatory motion of the electron in a laser field [77]:

$$
U_{p}=\frac{e^{2} E_{0}^{2}}{4 m \omega_{0}^{2}} \propto I_{0} \lambda_{0}^{2} .
$$

In summary, from equations 2.13 and 2.14 it follows that the maximum achievable high harmonic frequency can be increased by increasing the ioni- 

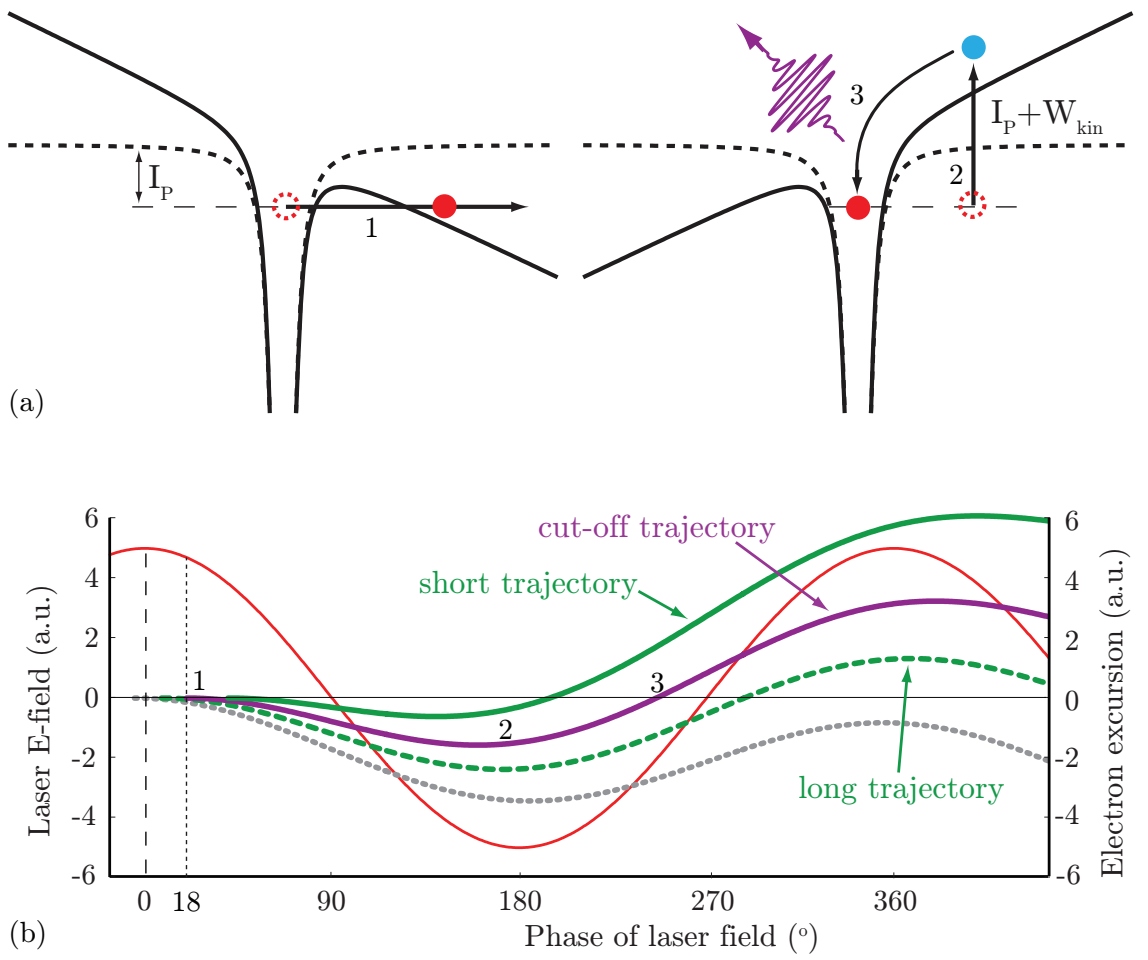

Figure 2.2: (a) The semi-classical three-step model explains the single atom response of HHG: 1) The electron is ionized through tunneling. 2) The electron propagates in the continuum and is accelerated. 3) The electron recombines and emits radiation with a frequency that equals the ionization potential plus the kinetic energy. (b) Different instants of ionization with respect to the laser field (red line) result in different electron excursion trajectories (other lines).

zation potential of the gas, $I_{p}$, the fundamental wavelength of the drive laser pulse, $\lambda_{0}$ and its intensity, $I_{0}$.

The described process of harmonic generation occurs twice during each optical laser cycle, and thus possesses inversion symmetry. From Fourier theory it can be understood that, in such a situation, the radiation consists of only odd harmonics of the fundamental laser frequency. Furthermore, every half of an optical cycle, the radiation is emitted during ultrashort intervals, shorter than half of the drive laser period, and hence in the form of an attosecond pulse train $(\mathrm{APT})$. 
As mentioned above, harmonic frequencies in the plateau region result from a short and a long electron trajectory, leading to the emission of radiation at different instants in time with respect to the drive laser phase. In addition to this phase term associated with the different recombination times, $t_{r}$, there is also a phase term related to the motion of the electron wave packet in the continuum. The calculation of this phase requires a quantum mechanical description [73]. As with transitions between bound atomic states, the time-dependent wave function is determined and evaluated to obtain the time-dependent atomic dipole moment. The power and phase spectrum of the emitted electromagnetic wave is then obtained by taking the Fourier transform of this dipole.

The phase acquired by the electron wave function is transfered to the photon pulse through the oscillating dipole [72]. The phase of the generated light at a particular frequency is called the intrinsic phase, $\phi_{i n t r}$. It is related to the propagation time, $\tau$, of the electron in the continuum and its kinetic energy, and thus to the drive laser intensity [78]. This intrinsic phase is approximately linearly dependent on the drive intensity [79]. Thus, the phase of harmonic order $q$ due to the short or long quantum path trajectories $(i=1,2)$ becomes

$$
\phi_{q, i}=q \omega t_{r, i}-\phi_{\text {intr }, i} \approx q \omega t_{r, i}-\tau_{i} U_{p}=q \omega t_{r, i}-\alpha_{i} I(r, z, t) .
$$

Here, $\alpha_{i}$ is the slope of the phase as a function of the intensity for either one of the two quantum path components, and is larger for the long trajectories. It can be seen from equation 2.15 that light waves of equal frequency generated by the long and short trajectories have different phases and consequently interfere non-constructively. The absolute values of both phases are quite large, especially for the long trajectories; in the order of dozens to hundreds of radians. When taking into account that the laser intensity varies during the pulse and that (following the $q$-dependence in equation 2.15) different frequencies are generated at different moments in time, this results in a chirp in the harmonic pulses [80].

In addition, the relation 2.15 implies that long trajectories have a stronger intensity-dependence of the phase. The phase of harmonics due to the short trajectories does not vary much with laser intensity. Consequently, short trajectories result in a longer coherence time of the harmonics and less divergent beams when compared to long trajectories. Therefore, it would be beneficial to select only radiation generated via the short trajectories. This can be achieved by placing an appropriate aperture in the harmonic beam or by using a hollow-waveguide for HHG, both of which act as a spatial filter and suppresses the radiation from the long trajectory harmonics $[50,81,82]$.

So far we have only considered the physical properties of HHG on the microscopic level, and this already explains some of the most basic features of the process. In experiments, however, harmonics are generated on a more macroscopic scale, in media that consist of many atoms and also impose a dependence of HHG on macroscopic parameters, such as the length of the generating medium. 


\subsubsection{Macroscopic description}

In order to efficiently generate harmonics from the entire propagation length through the nonlinear medium, it is essential to provide a constructive superposition of the harmonic fields emitted by all single atoms. This requires phase-matching, meaning that the phase velocity of the driving field has to match the phase velocity of the generated harmonic wave [49]:

$$
\Delta k_{q}=q k_{0}-k_{q}=0 .
$$

Here, $k$ denotes the wave vector. Unfortunately, due to optical dispersion, phase-matching is not easily obtained. In more detail, when considering HHG in a gas-filled (hollow waveguide) capillary, and, when the high drive laser intensity also leads to some ionization (plasma), there are three contributions that need to be balanced for phase-matching. These are the positive dispersion by the neutral gas and the negative dispersion by the plasma and the hollow waveguide. Equation 2.16 then becomes [50,83]

$$
\Delta k_{q}=\frac{2 \pi q}{\lambda_{0}}\left(1-\eta_{i}\right) P \Delta n-P \eta_{i} N_{a t m} r_{e} \lambda_{0}\left[\frac{q^{2}-1}{q}\right]-\frac{u_{n m}^{2} \lambda_{0}}{4 \pi a^{2}}\left[\frac{q^{2}-1}{q}\right],
$$

where the three terms on the right-hand side correspond to the named contributions. Here, $P$ is the pressure (in atm), $\eta_{i}$ is the ionization fraction, $N_{a t m}$ is the electron number density at atmospheric pressure and $r_{e}$ is the classical electron radius. $\Delta n$ is the difference between the refractive indices of the neutral gas at atmospheric pressure for the fundamental IR wave and the harmonic light. $a$ is the capillary inner radius and $u_{n m}$ is a dimensionless number which is specific for the particular waveguide mode of propagation. As an example, the value is 2.405 for the lowest order mode $u_{11}$.

The main conclusion from equation 2.17 is that phase-matching can be obtained when the ionization is held sufficiently low $(<10 \%$ for Xe and $<0.5 \%$ for $\mathrm{He}$ ), by tuning the pressure such that the neutral gas dispersion compensates for the plasma dispersion and the dispersion due to the hollow waveguide. In a theoretical analysis performed by Naumov et al., the optimum pressure for phase matching was determined for the $27^{\text {th }}$ harmonic from Xe, Kr, Ar, Ne and $\mathrm{He}$ in a hollow-fiber and they found that the optimum pressure increases with the named sequence of gases [84]. Slightly increasing the ionization resulted in broader phase-matching pressure peaks at a higher pressure, as was observed in experiments by Durfee et al. [83]. In addition to a high yield under phase-matched conditions, the XUV beam profile showed improved spatial coherence $[49,83,85]$. However, higher levels of ionization give rise to a strong (dominant) plasma dispersion that makes pressure-tuned phase-matching impossible. When the phase-matching condition is not fulfilled, the phase slip between the fundamental and harmonic waves will result in destructive interference after the so-called coherence length, which is given by $L_{c o h}=\pi / \Delta k[86]$. For ionization fractions of more than $10 \%$, the coherence length is typically less than $1 \mathrm{~mm}$ and decreases with the order of the harmonics, $q$. As a consequence, 
in order to maintain phase-matching for efficient conversion, the light intensity of the drive laser is to be restricted to a range where ionization is low. Hence the maximum harmonic frequency that can be generated is limited to much below what seems possible according to the cut-off rule. Possible methods to overcome this problem are discussed in the following subsection.

Another macroscopic effect that has to be taken into account is the absorption of the harmonic radiation by the generating medium itself [87]. This limits the useful length of the medium to approximately 10 times the absorption length, depending on the type of medium and the harmonic frequency. For example, the absorption length is $1 \mathrm{~mm}$ for the $45^{\text {th }}$ harmonic order in 60 mbar of neon. Maximum output would thus be obtained with a medium length of $1 \mathrm{~cm}$, which is easily achieved with a gas-filled hollow waveguide (capillary).

\subsubsection{Enhancement of HHG}

From experiments performed in the past it is clear that a gas-filled hollow waveguide is one of the most suitable geometrical configurations for HHG regarding conversion efficiency and output beam quality [19]. This is due to an enlargement of the interaction length [49]. In addition, the spatial filtering, associated with the long propagation in the capillary, selects light emission originating from a single recollision trajectory, leading to an increased temporal coherence $[49,50]$. Furthermore, the drive laser field forms a stable eigenmode (quasi-plane wave) along propagation, which results in a smaller divergence of the harmonic beam in comparison with free focusing in a gas jet $[42,49]$. For these reasons we decided to generate high harmonics by using gas-filled capillaries in our experiments.

There are several methods to further increase the output efficiency and the cut-off frequency using a capillary. In this section, quasi-phase-matching (QPM) techniques and an approach on the microscopic level, which we will refer to as Harmonic Excitation (HEx), will be discussed. The reason for this discussion is that our experiments aim to investigate two novel approaches to enhance the HHG output, namely via the extension of HEx to other systems and combine this technique with QPM. In this thesis we present the concepts and describe the experimental preparations, however, the actual demonstration will be performed after completion of this thesis. Other enhancement techniques which have been demonstrated already, for example, adaptive control and polarization gating [50], may then be added for further improvement. At that time, the recently discovered technique of plasmonically enhanced HHG [88], will be added as well. For this we have prepared several samples of metallic nano-structures in collaboration with other research groups associated with the $\mathrm{MESA}^{+}$Institute for Nanotechnology at the University of Twente (dr. ir. H. L. Offerhaus and prof. dr. J. L. Herek from the Optical Sciences group and prof. dr. ir. J. Huskens from the Molecular Nanofabrication group) and with the Institut de Ciències Fotòniques (prof. dr. J. Biegert from the Attoscience and Ultrafast Optics group). 


\section{Optimization of the cut-off rule}

Equation 2.13 for the cut-off rule appears to suggest that one could extend the cut-off by increasing the ponderomotive potential simply by increasing the drive laser intensity. However, the applicable intensity is limited because of the laser-induced ionization and, as a result, poor phase-matching conditions, as discussed in the previous section. Also, high drive intensities beyond a few times $10^{15} \mathrm{~W} / \mathrm{cm}^{2}$ would lead to an almost complete ionization of the atoms, thereby extinguishing the nonlinear atomic response. The cut-off rule, via equation 2.13, also suggests that simply increasing the drive laser wavelength would increase the cut-off [89]. However, long drive wavelengths also reduce the harmonic yield drastically because the yield scales with the driver wavelength as $\lambda_{0}^{-5.5}[90,91]$.

The cut-off rule also predicts higher-energy photons for higher ionization potentials. Indeed, this was experimentally observed. For example, when using helium compared to xenon, the cut-off increases [19]. Another possibility is to use ions as the medium for HHG instead of atoms. An additional advantage of using ions is that they can withstand higher intensities, which further increases the cut-off frequency. A central problem, however, is that poor phase-matching due to the strong plasma dispersion has not been solved yet and requires novel approaches to QPM, which will be discussed below. A less fundamental problem with HHG in ions appears when utilizing the drive laser pulse itself to generate the ionic medium, because this leads to a significant loss of the pulse energy and to ionization-induced defocusing and thus to a reduced drive intensity.

Ionization-induced losses and defocusing can be overcome by using a separate ionization mechanism, such as pre-ionization with a discharge ignited in the hollow waveguide capillary before the drive laser arrives. In this way, the harmonic cut-off has been experimentally increased to much higher harmonic orders than possible from atomic argon, krypton and xenon [92,93]. This discharge technique also yielded an increase of the harmonic flux also of the plateau harmonics by several orders of magnitude when compared with harmonic generation from laser-produced ions in a gas-filled hollow waveguide [94].

\section{Harmonic Excitation}

An optimization via the cut-off rule as described above is based on arguments regarding the microscopic response. Another promising method to enhance the output on the microscopic level is to increase the probability of the tunnelionization, the first step of the recollision model, via simultaneous irradiation of the atom with an additional light wave, in particular, an XUV harmonic field, with a frequency that is resonant with an excited state of the atom [59]. We will refer to this method as "Harmonic Excitation" (HEx), instead of the suggested name "Dramatic Enhancement" by the authors of [59].

The principle of HHG by HEx is schematically shown in figure 2.3. Once the electron makes the transition to a real (resonant) or virtual (near-resonant) 
state by absorbing a photon from the additional light field (1a in the figure), the tunneling barrier significantly reduces compared to the standard situation of the electron being in the ground state. Thus the probability of ionization increases $[95,96]$. The additional excitation can occur by single-photon excitation using a sufficiently short wavelength (e.g. a high harmonic XUV photon), or by stepwise and multi-photon excitation involving longer wavelengths (e.g. an XUV photon plus an IR photon). The remaining steps of HEx (2 and 3 in the figure) are analogous to the standard three-step model.

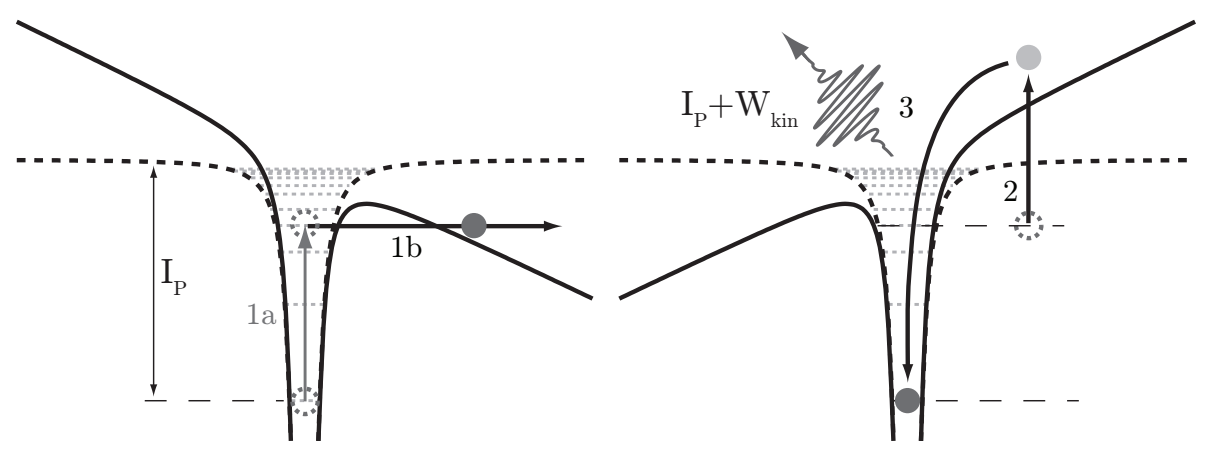

Figure 2.3: In the semi-classical three-step model for Harmonic Excitation (HEx) the first step is divided in two. In this case the drive laser is too weak to substantially modify the Coulomb potential to facilitate tunneling ionization of the electron in the ground state. By exciting the electron to a (near-) resonant state with an XUV harmonic (1a), the rate of tunnel ionization (1b) is enhanced. The second and third step in the model are the same as before.

Theoretically [95,96], this method predicts an enhancement for HHG of more than 10 orders of magnitude when irradiating a single $\mathrm{He}^{+}$ion with a fundamental wavelength of $800 \mathrm{~nm}$ and the $13^{\text {th }}$ or $27^{\text {th }}$ harmonic. However, this prediction does not take into account any macroscopic effects, such as absorption. When considering such effects, it was theoretically shown that the enhancement remains intact for short propagation lengths with factors up to $10^{6}$ [97]. These qualitative predictions of harmonic excitation were made assuming that the excited states have relatively long lifetimes compared to the period of a laser cycle. Therefore, the relative phase between the fundamental wave and the harmonic pulse has no effect on the efficiency.

Experimentally, an increase in efficiency of more than 3 orders of magnitude was obtained, by Takahashi et al., for the $27^{\text {th }}$ harmonic order with a gas mixture of helium and xenon, as compared to helium only [59]. In this experiment, a gas cell and a loose-focusing configuration was used at relatively 
low gas pressures (few Torr). The working principle of this approach, with a gas mixture, is that the harmonics from Xe, up to order 15, provide the additional light wave and excite electrons in He to assist tunnel-ionization from a virtual state of He. The used intensity of the harmonic pulse was estimated to be much lower than the fundamental laser pulse $\left(10^{11} \mathrm{~W} / \mathrm{cm}^{2}\right.$ as compared to $10^{14} \mathrm{~W} / \mathrm{cm}^{2}$, with a typical intensity of $8 \cdot 10^{9} \mathrm{~W} / \mathrm{cm}^{2}$ for the $15^{\text {th }}$ order). Note that harmonic orders higher than the $15^{\text {th }}$ order were generated as well but these photons do not contribute to HEx since these photons can induce XUV ionization of He directly. In this experiment, an optimum mixing ratio of Xe to He of about 0.08 was found.

HHG via HEx is especially useful in increasing the harmonic cut-off when the drive laser intensity is limited, e.g. due to technical limitations, meaning that the atom has an ionization potential that is too large to directly facilitate tunnel-ionization with the given drive laser field. Also, this method makes it possible to enhance the harmonic yield in the spatial wings of the drive laser pulse, thereby increasing the total output.

HEx has only been experimentally verified with neutral atoms so far. However, the cut-off frequency can be further increased using ions instead of neutral atoms, as mentioned before. HEx from ions seems, as the theoretical predictions of Ishikawa [95,96] and Schiessl [97] suggest, a promising method to further enhance the harmonic efficiency of the cut-off harmonics, but such experiments have not yet been performed. As mentioned in the introduction of this chapter, one of our goals is to demonstrate HEx in ions. To prepare the experimental conditions for such a demonstration, we decided to attempt HEx in a capillary without a discharge and thus with atoms, before applying a discharge to provide ions for HEx in ions. However, a central problem is that using ions leads to poor phase-matching due to the strong plasma dispersion. In order to overcome this problem and be able to further enhance the efficiency of HEx in ions on a macroscopic level, we will implement quasi-phase-matching (QPM) techniques in our experiments. Before a description of QPM techniques is presented in this section, another processes on the microscopic level should also be considered as a further preparation step. This is related to HEx and may occur simultaneously to or even compete with HEx.

The method to be considered is the, so-called, attosecond pulse train (APT) controlled HHG [98]. This is also a method of HHG enhancement which increases the ionization probability with XUV harmonics. In this case, the gaseous medium is simultaneously irradiated by an IR pulse and a copropagating attosecond pulse comprised of XUV harmonics which have photon energies that are, in contrast to HEx, higher than the ionization potential of the atom. Therefore, single-XUV-photon ionization can occur, and will be the dominant process over tunnel-ionization when the intensity of the attosecond pulse is sufficiently high. Adjusting the timing of the attosecond pulse with respect to the phase of the IR drive laser, controls the moment of ionization and therefore permits the selection of the harmonic output by either the short or long quan- 
tum trajectories. The advantage of this control is that the non-constructive interference of high harmonic radiation generated via both trajectories, due to their different harmonic phases, is reduced and, as a result, the output is increased (see subsection 2.2.1). In addition, the coherence properties of the harmonic beam will be improved when radiation due to the short trajectories is selected (see subsection 2.2.1).

A disadvantage is that APT controlled HHG requires an intense attosecond pulse to make single-photon ionization dominant over tunnel-ionization. Unfortunately, obtaining an intense APT with an adjustable phase with respect to the fundamental wave is experimentally quite complicated. In an experiment performed by Biegert et al. [82], harmonics from a Xe-filled capillary were focused together with the fundamental pulse into a He gas jet. They attained an enhancement of the harmonic yield of a modest factor of 5 , which is much lower than the three orders of magnitude that was achieved with HEx, as was mentioned above.

As mentioned above, APT controlled HHG will be dominant over HEx when the intensity of the attosecond pulses is sufficiently high, which means that the enhancement due to HEx will be reduced. So, in order to facilitate HEx, one should avoid single-XUV-ionization. This can be done by regulating the intensity of the attosecond pulse, or, in the example of an He-Xe mixture, by adjusting the drive intensity such that from Xe only high harmonics are generated with photon energies below the ionization potential of He.

\section{Quasi-Phase-Matching}

Without phase-matching between the fields of the drive laser and the generated harmonics, as mentioned in subsection 2.2.2, there is no constructive build-up of the harmonic signal in the HHG medium over propagation distances longer than the coherence length, $L_{c}$. This is schematically depicted in figure 2.4 , as the solid black curve.

To prevent destructive interference after one coherence length, the wellknown technique of quasi-phase-matching (QPM) [99] can be attempted. In QPM, the phase-mismatch is adjusted in the regions of out-of-phase generation or the harmonic generation is locally suppressed. This is done via a spatially periodic modulation of the nonlinear coefficient, either in phase or in amplitude. It can be shown that the modulation introduces an additional wave vector, $K$, to the total wave vector balance (equation 2.16) $[99]^{1}$ :

$$
\Delta k_{q}=q k_{0}-k_{q} \pm K
$$

Here, $K$ may be adjusted to a desired value by choosing a proper modulation period. Specifically, to achieve QPM with $\Delta k_{q}=0$, the modulation is to be chosen to match with the coherence length according to $K=2 \pi / \Lambda$, with $\Lambda=2 L_{c}$ as indicated in figure 2.4 .

\footnotetext{
${ }^{1}$ Here, first-order QPM is presumed
} 


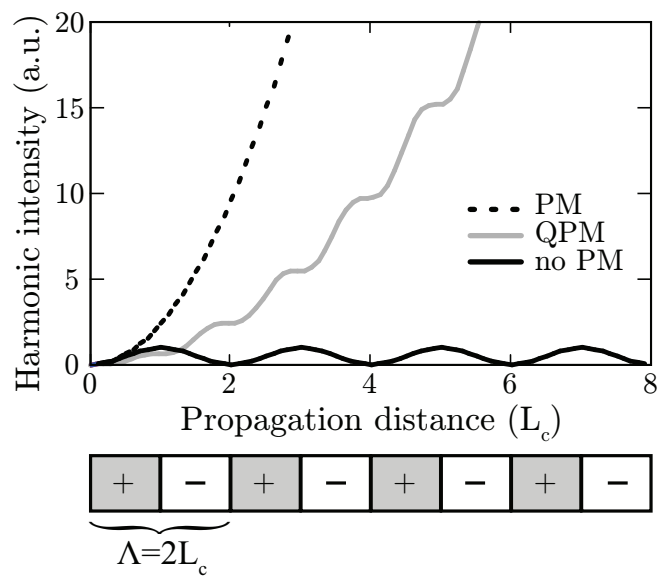

Figure 2.4: Harmonic intensity as a function of the propagation distance in three situations. Without phase-matching (black solid curve), harmonic build-up in regions of proper phasing of the generated radiation with the drive laser $(+)$ is canceled after one coherence length, $L_{c}$, by out-of-phase generation (-). When phase matching (dashed black curve) cannot be realized, so-called quasi-phasematching (QPM, grey curve) can be attempted. It can be seen that QPM leads to the desired growth of output with increasing propagation distance, just as PM besides a certain constant factor.

QPM was first implemented in non-linear crystals [99], because, there, a permanent modulation of the nonlinear coefficient is possible. An example of this is a technique called periodic poling. However, such techniques cannot be applied to gases or a plasma, because, here, the atoms or ions are in a rapid thermal motion leading to a homogeneous (non-modulated) state of the medium. Instead, a modulation of the density of the medium or the electric field of the drive laser is conceivable. Also, a modulation of the intrinsic phase of the harmonic field may be used via a spatially modulated drive intensity (see equation 2.15). Note, that even small modulations of the driving electric field can induce large phase shifts.

Unfortunately, such methods of QPM for HHG are not easy to implement and they also have their limitations with respect to the harmonic orders that can be phase-matched. In particular, harmonic orders with photon energies above a keV are associated with very short coherence lengths, in the order of micrometers or less (see section 2.2.2, using equation 2.17). In order to deduce possible techniques that can be relatively easily applied in our experimental setup using gas-filled capillaries and, eventually, discharge capillaries, a few techniques of QPM that have previously been suggested for HHG in such ge- 
ometries will be discussed.

The first example of QPM that proved to be effective in enhancing the conversion efficiency of HHG, was based on a gas-filled hollow waveguide, but where the inner diameter was modulated along the propagation distance [76,100]. The modulation leads to a periodic change of the drive laser intensity. Using such a modulated capillary, at a relatively low intensity, the intensity is modulated such that the intensity is only high enough to generate high harmonics in the narrow sections of the capillary. This method is especially effective for harmonics in the cut-off region [76]. Also, a modulated waveguide was used effectively at high laser intensities where the medium was (almost) fully ionized [100]. In that experiment, the periodic change in intensity was sufficient to adjust the intrinsic phase of the generated harmonic fields in order to achieve QPM. In this way an enhanced output in Ne was achieved of an order of magnitude for a photon energy of $225 \mathrm{eV}$ and an extension of the cut-off to $284 \mathrm{eV}$.

However, as mentioned before, high drive intensities lead to ionizationinduced losses and defocusing, which can be overcome by using a pre-ionized medium created in, e.g., a discharge capillary [92]. Such a discharge capillary has been considered with a periodic modulation of the inner wall to attempt QPM [101]. However, numerical calculations showed that the spatial modulation of the plasma channel that is formed (and guides the laser beam) is too weak to be used for QPM. An additional limit to QPM is that to phase match harmonic radiation with progressively shorter wavelengths requires that successively smaller modulation periods are to be realized. This is increasingly difficult to achieve, which limits the maximum harmonic frequency that can be phase matched. Nonetheless, it is predicted that the efficiency of harmonic radiation with photon energies up to a keV may be enhanced by QPM with gasfilled modulated hollow waveguides even with laser-induced ionization [100].

A second approach to QPM is to modulate the drive laser intensity by utilizing mode beating between multiple propagation modes in a hollow waveguide [102]. In this method of multi-mode QPM (MMQPM), part of the pulse energy is converted into higher-order modes [103]. This can be achieved when a pulse with non-Gaussian spatial profile (e.g. a flat-top beam), or a Gaussian beam with a focal waist $w_{0}$ that deviates sufficiently from 0.64 times the capillary inner radius $a$ (the optimal ratio to couple the pulse only into the fundamental mode of the capillary), is coupled into the waveguide [103]. Beating between the modes leads to a significant intensity modulation with a short periodicity. This periodicity becomes smaller when the number of modes increases, which is necessary to achieve QPM for an increasing order of the high harmonics. With MMQPM and utilizing an argon-filled capillary with a length of $1 \mathrm{~cm}$, Zepf et al. [102] showed experimentally an efficiency enhancement of more than 3 orders of magnitude for harmonics in the water window $(\approx 4 \mathrm{~nm})$. Actually, in that experiment the highest order harmonics resulted from laserproduced ions. It appears that MMQPM may be used in capillary discharges, because higher-order modes can also be excited in a plasma channel formed 
by such a discharge. The advantage of this approach is that it is easier and more flexible than the method using a modulated waveguide. It is important to consider, however, that higher order modes have higher propagation losses. In order to fully exploit the medium, longer capillaries with lengths of up to 10 times the absorption length have to be used to further increase the harmonic output, as described above. Further investigation is necessary to decide if this method can be extended to capillaries longer than $1 \mathrm{~cm}$.

A third method to be considered for QPM in waveguides, is suppression of harmonic build up in out-of-phase regions using a counter-propagating pulse train with pulses of appropriate width and separation $[90,104]$. In regions where the drive laser intersects with a relatively long and weak counter-propagating pulse, there is a small modulation of the laser intensity with a periodicity of half the laser wavelength. As a consequence, the rapid harmonic phase modulation, which is faster than the coherence length, prevents local phase matching and harmonic build up. If the width of the counter-propagating pulses matches the coherence length, harmonic generation in the out-of-phase regions can be suppressed and QPM is achieved. In addition, this technique has the potential to be adjustable during harmonic generation. One may vary the pulse duration and separation in the pulse train, e.g., to compensate for the dynamically changing phase-mismatch due to the time-varying laser intensity during propagation or to tune the phase matching toward desired wavelength ranges. When considering typical experimental parameters, this method requires a counterpropagating pulse train of at least several pulses of picosecond duration and spacing. However, experimentally such a pulse train is difficult to produce. Nonetheless, by applying a counter-propagating pulse train of 4 pulses, an enhancement by a factor of 600 was achieved in argon for the $41^{\text {th }}$ harmonic order [90]. The advantage of this all-optical technique of QPM is that it is also suitable for HHG in ions.

All these techniques of QPM are applicable for XUV harmonic orders of energies below a keV, from which MMQPM seems to be the easiest and most effective method realizable for HHG in gas-filled capillaries and capillary discharges. To realize QPM also for harmonics with photon energies above a keV, there are only theoretical suggestions so far, such as grating-assisted phasematching (GAPM) [105]. This method relies on compensating for the intrinsic phase of harmonics generated in the destructive regions instead of suppressing the harmonic generation. This could be achieved by injecting a relatively weak and long counter-propagating laser pulse (quasi-continuous wave) in addition to the short drive laser pulse. The field of this second beam induces a sinusoidal modulation on the drive laser intensity with a periodicity of half of the second laser wavelength. Because the large intrinsic phase of the high harmonics is strongly dependent (proportional) on the total intensity of the superimposed lasers, a small intensity modulation is sufficient to adjust the phase by $\pi$ radians as required for QPM. Hence, by properly adjusting the intensity modulation, harmonics that would be generated in the out-of-phase regions are shifted into phase and interfere constructively. In this way, numerical calculations by Cohen 
et al. [105] predict an enhancement by a factor of $4 \cdot 10^{4}$ of the $647^{\text {th }}$ harmonic in a pre-formed plasma waveguide that contains doubly ionized neon ions at a pressure of 70 Torr, using a $20 \mathrm{fs}, 800 \mathrm{~nm}$ drive laser pulse with peak intensity of $5.5 \cdot 10^{13} \mathrm{~W} / \mathrm{cm}^{2}$ and a weak counter-propagating beam of $3.5 \cdot 10^{8} \mathrm{~W} / \mathrm{cm}^{2}$ with a wavelength of $1600 \mathrm{~nm}$. In order to generate harmonics with energies above a keV via this method, an additional laser beam at around $1.6 \mu \mathrm{m}$ is to be made available, coupled into the capillary from the rear side, such that the high harmonic beam can still be coupled out of the capillary.

\subsection{Discussion}

HHG from atoms has proven itself to be a highly important technique to generate coherent ultrashort pulses of XUV and soft X-ray. Meanwhile, this technique has found several applications, such as X-ray microscopy and interference lithography, and it has opened the door to attosecond science. To further widen the scope of such applications and to enter novel, unexplored regimes, it is of central interest to identify approaches that can increase both the harmonic yield and the generated frequencies.

We intend to experimentally investigate enhancement techniques for HHG. In particular, three enhancement techniques which have been described in this chapter will be implemented in our experiments. First, we will use ions to exploit their higher ionization potential than neutral atoms. This should allow us to apply higher laser intensities and extend the harmonic cut-off via both $U_{p}$ and $I_{p}$ in equation 2.13. Secondly, harmonic excitation (HEx) will be applied in a novel setting, as described below. Combining these two methods would enable the performance of harmonic excitation of ions. In addition, QPM by multi-mode beating seems to be the most promising method to further enhance the harmonic output from ions in the future.

Based on these considerations, we will investigate HHG from ions via harmonic excitation by utilizing a capillary discharge. Two sections could be used: a first section with neutral gas from which the high harmonics excite the ions in a second section obtained by a discharge. In the next step we will implement QPM to compensate the phase-mismatch induced by the plasma dispersion. We have chosen to implement such QPM via the multi-mode beating (MMQPM) because this seems to be relatively straightforward in terms of technical implementation in our capillary discharges (e.g. available from experiments on laser wakefield acceleration in our group [47]).

To implement the demonstration experiment outlined above, a corresponding experimental setup, that employs a suitable fraction of the output from the multi-TW laser system at LPNO, had to be devised. Using this plan as a guide, the first step of the research and one of the main goals of the thesis, is to devise, construct and bring into operation a complete setup for HHG and related diagnostics. The construction and testing of harmonic generation with gas-filled capillaries, which will be described in chapter 4 . The advantages and potential improvements of our apparatus will be discussed. For example, in 
order to prevent re-absorption of the harmonic radiation behind the capillary, a special design of the capillary was made to allow for differential pumping. Also, our setup enables us to characterize the harmonic spectrum as well as the harmonic beam profile. It should be noted that the advantage of making use of the multi-TW laser at LPNO, which is optimized for experiments on Laser Wakefield Acceleration, means that there are some additional challenges regarding the construction of the experiment and the measurements of HHG. For example, the detection of high harmonic radiation and recording harmonic spectra had to be successful in spite of the relatively low repetition rates (0.1 to $10 \mathrm{~Hz}$ ), which is typical for the amplifier chain of such laser systems.

To demonstrate the working of our apparatus, well-known experimental results have been repeated, and also the experimental implementation of harmonic excitation (HEx) in gas mixtures in a guided-wave geometry will be presented in chapter 4 . 


\section{Theory of high-order harmonic generation and ion acceleration from solids}

High harmonic generation from gaseous media (HHG), as described in the previous chapter, is one example of the many high-intensity laser-matter interactions that can be observed at low densities of the generating medium. The development of multi-terawatt lasers also opened up a range of other exciting phenomena with targets of much higher density, such as high-order harmonic generation (HOHG) and ion acceleration from solids. We performed experiments to investigate such processes with the laser at PHI, as will be discussed in chapter 5. In particular, we investigated the interaction with foils as thin as a few nanometers to enter the so-called transparent regime for the first time. To provide a better understanding of the experimental results, a theoretical description of HOHG and ion acceleration is presented in this chapter. However, both processes involve rather complex interactions between the laser and the plasma. Therefore it is instructive to start with some basic considerations of high-intensity laser-solid interactions.

Any solid target (whether opaque or transparent) when illuminated with a high-intensity laser pulse will be rapidly ionized and within a few laser cycles a reflective (so-called overdense) plasma wall is created at the surface of the target [62]. Various types of physical processes, such as emission of radiation or the acceleration of particles, can take place during the interaction of the remaining part of the incident laser pulse with this overdense plasma. However, a common issue in these processes, including HOHG and ion acceleration, is the absorption of radiation and the subsequent energy transfer to the plasma. 
This absorption and energy transfer are quite different from the corresponding standard processes at low intensities. This is because, at high intensities, one needs to consider that absorption depends strongly on the temperature of the plasma, the density of the plasma and the density gradient. In addition there is a strong dependence on all of the laser pulse parameters, such as the duration, the energy, the peak intensity, the contrast, the light frequency, the polarization and the angle of incidence.

Due to its ultrafast and strongly nonlinear dynamics, the absorption of highintensity light in an overdense plasma and the result of the absorption is much different from what is seen at lower densities or lower intensities. Absorption will lead to an ultrafast local heating of the plasma and the increased temperature results in a spatially dependent change of the absorption efficiency. Additionally, the high temperatures and high pressures that are generated result in a rapid expansion with fast changes of the density distribution. These modifications of the plasma in space and time introduce a considerable level of complexity to the processes [61], and so the interaction between electromagnetic radiation and overdense plasmas is still not well understood [106]. What is available so far are partly valid theoretical descriptions that include selected types of mechanisms responsible for absorption which are applicable for certain regimes of laser intensity and plasma density gradients.

In high-intensity laser-solid interactions two different phases of absorption occur [107]. During the first phase, at relatively low laser intensities in the rising edge, or the pedestal of the incident light pulse $\left(\approx 10^{11}-10^{15} \mathrm{~W} / \mathrm{cm}^{2}\right)$, plasma formation starts by optical field ionization and subsequent, so-called, collisional absorption results in progressing ionization and heating of the plasma. Here electrons driven by the electric field of the light collide with atoms or ions and thereby transfer energy to the plasma. In the second phase, at high intensities $\left(>10^{15} \mathrm{~W} / \mathrm{cm}^{2}\right.$ ), the thermal velocity of the electrons becomes too large to provide an effective collisional energy transfer, instead, so-called, collisionless absorption dominates. This will be described in subsection 3.1.3. A very special situation can be generated by applying ultrashort laser pulses that are optimized for a high contrast, as there is almost no collisional absorption due to the rapid temperature rise $[62,108]$. This thesis concentrates on such experiments and a theoretical description of this situation, of which only little is known. In particular, it turns out that even nanometer-scale plasma expansion has to be taken into account when irradiating extremely thin foils, even when using pulses optimized for ultra-high contrast, as will be discussed in chapter 5. For the interpretation of such experiments it is important to understand the effect of the contrast of a pulse and the resulting density gradient that is caused by plasma expansion, as described in the first section of this chapter.

In addition, the first section of this chapter describes in more detail several mechanisms involved in absorption in order to give insight into the physics behind high-order harmonic generation and ion acceleration. Because of the complex interplay between absorption and plasma expansion, the first section starts with a short description of plasma expansion and its description via the 
so-called density scale length, $L$, which is an important parameter for both HOHG and ion acceleration. The remainder of the first section is divided into subsections on collisional and collisionless absorption mechanisms for plasmas with a short scale length $(L / \lambda \lesssim 0.1)$ and a long scale length $(L / \lambda>0.1)$, with respect to the laser wavelength, $\lambda$. An important conclusion is that the laser pulse contrast, via the scale length, is a very critical parameter, as it sets the initial conditions for the interaction of the drive laser pulse with the plasma.

The physics of ion acceleration is described in the second section, with an emphasis on target normal sheath acceleration (TNSA), which is the dominant acceleration mechanism in our experiments. In particular, the theoretical prediction of ion acceleration in the transparent regime is discussed. This is relevant for our experiments with extremely thin foils (in the range of a few nanometers). The subsequent section is devoted to a theoretical description of HOHG in two different parameter regimes, called the relativistic oscillating mirror (ROM) model and the coherent wake emission (CWE) model.

\subsection{Absorption of laser energy by plasmas}

\subsubsection{Plasma expansion}

An important dynamic property of hot and dense plasma is its expansion into the vacuum, as this critically influences the interaction processes of the plasma with EM waves. The velocity of the expansion is driven by the electron pressure but limited by the inertia of the ions and thus determined by the electron temperature $k_{B} T_{e}$ and the ion mass $m_{i}$ as $[60,61]$ :

$$
c_{s}=\sqrt{\frac{Z k_{B} T_{e}}{m_{i}}} .
$$

Here, $Z$ is the number of free electrons per atom, and $c_{s}$ is referred to as the ion-acoustic velocity, also named the speed of sound in plasma.

For most experimental situations, where quasi-neutrality, $n=n_{e}=Z n_{i}$, is present [109], the expansion is well described by a time-dependent exponential profile of the plasma density $n$, where $n_{e}$ and $n_{i}$ are the electron and ion density, respectively;

$$
n=n_{0} \exp \left(-1-\frac{x}{c_{s} t}\right) .
$$

In this expression, the characteristic density scale length $L$ is defined as

$$
L \equiv-\frac{n}{\partial n / \partial x}=c_{s} t .
$$

For electron temperatures in the order of a few hundred $\mathrm{keV}$, which is typical for experiments with intense ultrashort laser pulses, the expansion velocity is about $0.1 \mu \mathrm{m} / \mathrm{ps}(100 \mathrm{~km} / \mathrm{s})$. 
If a high-intensity laser pulse with a low contrast is used, this means that the pedestal (typically tens of ps long) of the pulse will already ionize the target well before the main pulse arrives. This also means that the generated plasma will already have expanded over several micrometers from the formerly sharp surface of the target before the main pulse arrives. The term pulse contrast refers, not only to a pedestal before the main pulse, but also to all other types of light arriving before the main pulse, such as ASE or pre-pulses (see section 1.1). Consequently, the main pulse may propagate through a larger region of underdense plasma before it reflects at a surface beyond which the density is above the critical density. This surface is not flat and well-defined because of a variety of factors, for example, so-called Rayleigh-Taylor instabilities that cause rippling of the plasma [110]. As a consequence the efficiency of, e.g., high-order harmonic generation will be low, because high efficiencies require a flat, mirror-like surface, as will be described in section 3.3. In order to avoid too wide a plasma expansion and the related instabilities, high-contrast laser pulses are required for this process. Such laser pulses are also favored for ion acceleration, though, by utilizing relatively thick foils $(>\mu \mathrm{m})$, it is possible to generate fast ions from the rear surface of such foils also with low-contrast pulses [111].

\subsubsection{Collisional absorption}

In view of the pre-expanded state of the plasma, two mechanisms of collisional absorption of the main drive pulse will be discussed. The first is called classical collisional absorption, which occurs at long scale lengths $(L / \lambda>0.1)$. The second is called the normal skin effect that takes place at sharper plasma interfaces $(L / \lambda \lesssim 0.1)$. Both absorption mechanisms are most efficient below a laser intensity of the order of $10^{16} \mathrm{~W} / \mathrm{cm}^{2}$.

Classical collisional absorption (also referred to as Inverse Bremsstrahlung) starts as the incident electromagnetic wave propagates through the underdense plasma and causes the electrons to quiver with the optical field. The quiver energy of the electrons is transferred to the plasma through random collisions with atoms and ions, thereby increasing the thermal energy of the plasma and decreasing the intensity of the EM wave. During the collisions, atoms and ions are further ionized and the electrons colliding with ions are decelerated or deflected by Coulomb forces. This generates Bremsstrahlung which, in its turn, can ionize atoms $[61]^{1}$.

The efficiency of the overall energy transfer from the drive laser to the plasma increases with the frequency of collisions between the electrons and ions. The collision frequency grows with the electron density $n_{e}\left(\right.$ in $\left.\mathrm{cm}^{-3}\right)$, the inverse of the electron temperature $k_{B} T_{e}$ (in $\mathrm{eV}$ ) and, with the charge of the ion

\footnotetext{
${ }^{1}$ The light frequency of Bremsstrahlung depends on the velocity of the electron and the distance between the electron and the ion. A small interaction distance and a low thermal velocity lead to a large deflection angle and thus to the emission of high-energy photons.
} 
Theory of high-order harmonic generation and ion acceleration from solids

(number of free electrons per atom) $Z$, according to the following relation [62]

$$
\nu_{e i}=2.91 \cdot 10^{-6} Z n_{e}\left(k_{B} T_{e}\right)^{-3 / 2} \ln \Lambda \quad \mathrm{s}^{-1} .
$$

Here, $\Lambda$ is the ratio of the maximum and minimum distance between the electron and ion (so-called impact parameter $b$ ). The maximum distance $\left(b_{\max }\right)$ corresponds to the Debye length beyond which the individual ion is effectively screened. The minimum distance $\left(b_{\min }\right)$ is the classical distance of closest approach without capture (recombination) of the electron by the ion [61]. $\Lambda$ can be rewritten as $9 N_{D} / Z$, where $N_{D}$ is the number of electrons in the Debye sphere, given by equation 2.3 .

From equation 3.4 it can be seen that the collision frequency and hence the absorption efficiency increases with decreasing electron temperature. The efficiency also increases as the light wave propagates to higher densities, $n_{e}$, until it reaches the critical density, from where the wave is reflected back [61]. Furthermore, the absorption increases with increasing scale length, and depends on the polarization of the light. For example, for s-polarized light the maximum absorption occurs under normal incidence, while there is an optimum absorption for p-polarized light at an angle of incidence which increases as the scale length decreases [62]. A more detailed analysis of the dependency of the parameters on the absorption fraction is given in appendix B.1, where graphs of the absorption fraction at normal incidence at different scale lengths and laser intensities are shown. As can be seen from the graph in figure B.1, the absorption rapidly decreases with increasing intensity, due to a decreasing collision frequency, and with a decreasing scale length.

For the more step-like plasma density profiles $(L / \lambda \lesssim 0.1)$, which may be generated by high-contrast laser pulses at relatively low peak intensities of the main pulse $\left(<10^{16} \mathrm{~W} / \mathrm{cm}^{2}\right)$, another mechanism of collisional absorption, called Normal Skin Effect (NSE) can be expected to take place. At these intensities, the electron mean-free-path, which is defined as the thermal velocity divided by the collision frequency $\left(v_{e} / \nu_{e i}\right)$, is smaller than the skin depth (around $10 \mathrm{~nm}$ for fully ionized solid targets, see equation 2.10). Therefore, the electrons oscillate in the laser field and collide with ions within the skin depth layer, and the oscillation energy is locally thermalized [62]. The absorption via the NSE depends on the collision frequency but is in general less efficient than classical collisional absorption and is typically less than $10 \%$ of the incident laser energy. A more detailed description is given in appendix B.2 where the absorption fraction via the NSE is given and graphs of the absorption fraction are shown for two different laser intensities.

As mentioned before, during the interaction of a solid and a high-intensity laser, a plasma is formed with a density that is too high for the laser light to propagate (overdense plasma). The drive laser light penetrates the plasma to a short penetration depth exponentially decreasing in intensity and being totally excluded from the overdense region [61]. One might expect that plasma forma- 
tion is limited to the small region where the laser penetrates, however, plasma also can be formed at deeper regions. High-frequency radiation, e.g. X-rays via Bremsstrahlung and K-shell ionization [112], penetrate deeper (in the order of micrometers) into the target creating a plasma with low thermal energy (cold plasma). Furthermore, at intensities above approximately $10^{15} \mathrm{~W} / \mathrm{cm}^{2}$, a large number of high energy electrons (hot electrons) are produced via collisionless absorption mechanisms (see next section). These hot electrons can penetrate deep into the target and can instantaneously field-ionize neutral atoms [113].

\subsubsection{Collisionless absorption}

Collisionless absorption processes heat the faster electrons more effectively [114], and thereby lead to the heating of a fraction of the electrons to energies much higher than the initial plasma temperature $k_{B} T_{e}$ [62]. This results in a typical bi-Maxwellian electron temperature distribution, with a hot electron temperature $k_{B} T_{h}$ that depends on the mechanism of collisionless absorption. The generation of these hot electrons is important for processes such as HOHG and ion acceleration, and the efficiency of these processes increases with the number of hot electrons and their temperature, as will be described in sections 3.2 and 3.3. The number of hot electrons can be estimated by

$$
N_{h}=\frac{\eta \tilde{E}}{k_{B} T_{h}},
$$

where $\tilde{E}$ is the laser pulse energy and $\eta$ is the absorption fraction [115]. Consistent with equation 3.5, in experiments with current laser systems, the number of hot electrons generated per laser pulse is typically in the order of $10^{11}$ to $10^{13}$, which results in hot electron densities of about $10^{19}$ to $10^{22} \mathrm{~cm}^{-3}$, depending on the target and laser pulse parameters [116].

In the following paragraphs, several collisionless absorption mechanisms, which are expected to dominate during the generation of high-order harmonics and fast ions, will be described, for both short and long scale-length plasma gradients. Here we consider only cases of normal incidence and p-polarized light, as in experiments, because s-polarized light is of less interest due to its less efficient heating.

The first mechanism, called Resonance Absorption (RA) is a very efficient absorption mechanism for intermediate intensity levels $\left(10^{15}\right.$ to $\left.10^{17} \mathrm{~W} / \mathrm{cm}^{2}\right)$ and long plasma density gradients $(L / \lambda>0.1)$, when a p-polarized wave is obliquely incident.

Due to the non-zero angle of incidence, the traveling part of the light wave is reflected out of the plasma at a certain depth called the turning point where the density equals $n_{c} \cos ^{2} \theta$, which is less than the critical density [62]. The evanescent field, also called the tunneling field, penetrates deeper into the plasma and can reach the critical density. Due to the p-polarization of the wave, the electric field of the tunneling wave possesses a component along the density gradient 
that can directly excite electron oscillations at the critical density where the plasma response is in resonance $\left(\omega_{0}=\omega_{p}\right)[61,114]$ and initiate a plasma wave. The magnitude of this plasma wave grows linearly over a number of laser cycles and is eventually damped by collisions (at low intensities) or particle trapping and wave breaking (at high intensities) [62]. This resonance absorption, unlike the case with collisional absorption, couples laser energy to the collective motion of the plasma wave rather than to single electrons independently.

Resonance absorption depends on the scale length of the plasma gradient and the angle of incidence as can be seen in appendix B.3. It can also be seen that there is an optimum angle for resonance absorption that depends on the scale length. This is because on one hand, for a large angle of incidence, $\theta$, the turning point is far in front of the critical density which renders a weak tunneling field at the critical density. On the other hand, the longitudinal (normal) component of the electric field of the laser which drives the plasma wave decreases for smaller angles of incidence. The maximum coupling between the laser and plasma wave has been calculated to be approximately $60 \%$ in the limit of long scale lengths [62].

In the following paragraphs, a description is given on heating mechanisms at short scale lengths. Two mechanisms that occur at steep plasma gradients at intermediate intensities $\left(10^{15}\right.$ to $\left.10^{17} \mathrm{~W} / \mathrm{cm}^{2}\right)$ are Anomalous Skin Effect (AnSE) and Sheath Inverse-Bremsstrahlung (SIB). In both of these processes, the absorption is confined to the skin depth, similar to NSE discussed above. However, in the case of AnSE and SIB, the electrons gain higher energies and the mean-free-path is larger than the skin depth. This means that electrons that enter the skin depth and oscillate in the evanescent field of the laser, are reflected back into the plasma [64,117]. In particular, AnSE absorption is due to those electrons that have an insufficient initial velocity to reach the vacuum boundary. Electrons responsible for SIB absorption have higher energies but an unfavorable time of injection with respect to the laser phase and are reflected from the small Debye sheath near the plasma interface [108,117].

The absorption for AnSE and SIB is not large; approximately 5\% of the incident laser energy for normal incidence [62]. For obliquely incident, p-polarized light the absorption is slightly increased. The absorption process for p-polarization is also referred to as Sheath-Transit Absorption [64,118].

These two absorption mechanisms arise mainly during laser-plasma interactions with ultrashort laser pulses of intermediate intensities, where the light pressure is smaller than the plasma pressure $\left(2 I_{0} / c<n_{e} k_{B} T_{e}\right)[62]$. However, both processes can still occur at higher intensities of the pulse, most likely during the last half of the interaction, after the peak of the pulse has heated up the plasma [64].

However, in high intensity $\left(>10^{16} \mathrm{~W} / \mathrm{cm}^{2}\right)$ situations, the most efficient absorption related to $\mathrm{HOHG}$ and ion acceleration comes from two other effects [108]. The first is called the Brunel effect (also vacuum heating or "not 
so resonant" resonant absorption) [119]. The second effect is called Relativistic $\mathbf{v} \times \mathbf{B}$ heating.

The Brunel effect denotes electrons that oscillate along the field component of p-polarized laser light parallel to the plasma density gradient (target normal); similar to resonance absorption described above. However, for the Brunel effect, the light pressure is larger than the plasma pressure ${ }^{2}\left(2 I_{0} / c>n_{e} k_{B} T_{e}\right)$ in a situation where the density gradient is almost step-like (a short scale length $L / \lambda \lesssim 0.1)$. This means that electrons near the surface are pulled out by the strong laser field into the vacuum, well beyond the thermal Debye sheath, and hence no plasma wave can be resonantly excited by the laser field directly [62]. In the vacuum the electrons are directly exposed to the field and these electrons gain a large velocity $\left(v_{o s}=e E_{L} / m_{e} \omega\right)$ and hence energy (hot electrons). As the field decreases and reverses its direction in the other half of the optical cycle, the hot electrons are pushed back into the plasma ${ }^{3}$. Because of the small skin depth of the laser field, the electrons travel unhindered into the target (where they are called Brunel electrons) until their energy is eventually transfered to the plasma through collisions [62]. These hot electrons removed from the surface are replaced by new cold electrons from the bulk plasma. This electron current is called cold return current and is pointed in the opposite direction of the Brunel electrons [106].

The amount of energy that the hot electrons gain depends on their velocity and phase of excursion with respect to phase within the optical cycles of the laser light. For instance, electrons that move further into the vacuum return to the plasma with larger velocities, and eventually overtake electrons that had smaller excursions and were pushed back slightly earlier with smaller velocities. Via this mechanism, as will be described in section 3.3.2, a fraction of the Brunel electrons form an attosecond electron bunch, which proves to be important for high-order harmonic generation via coherent wake emission [20].

When quantitatively modeling the motion of electrons, the described effect of Brunel absorption can be clearly recognized in a phase-space density graph. An example is shown in figure 3.1, taken from Thaury [20]. The figure shows, in two plots, the velocity (momentum) distribution of electrons in space (onedimensional) at different steps in time (step 1 to 6 occurs during one optical laser cycle). The false-color code indicates the electron density (red and blue correspond to a high and low density, respectively). The laser is incident from the left side. When stepwise following the electron dynamics, it can be seen that at step 4, electrons with different velocities become concentrated at the same position in space and form an attosecond bunch [120].

For the absorption fraction of the Brunel effect which determines the number of hot electrons generated, an analytical expression was proposed by Brunel

\footnotetext{
${ }^{2}$ For an electron density of $10^{23} \mathrm{~cm}^{-3}$ and a temperature of $150 \mathrm{eV}$, the threshold intensity is $3 \cdot 10^{16} \mathrm{~W} / \mathrm{cm}^{2}$.

${ }^{3}$ More correctly: some electrons are pushed back as the field decreases through strong space-charge electrostatic fields that shield the plasma from the laser field (Brunel absorption), and some electrons are pushed back by the electric field directly (vacuum heating) [108]. In general these two terms are mixed up and refer to both situations.
} 
Theory of high-order harmonic generation and ion acceleration from solids
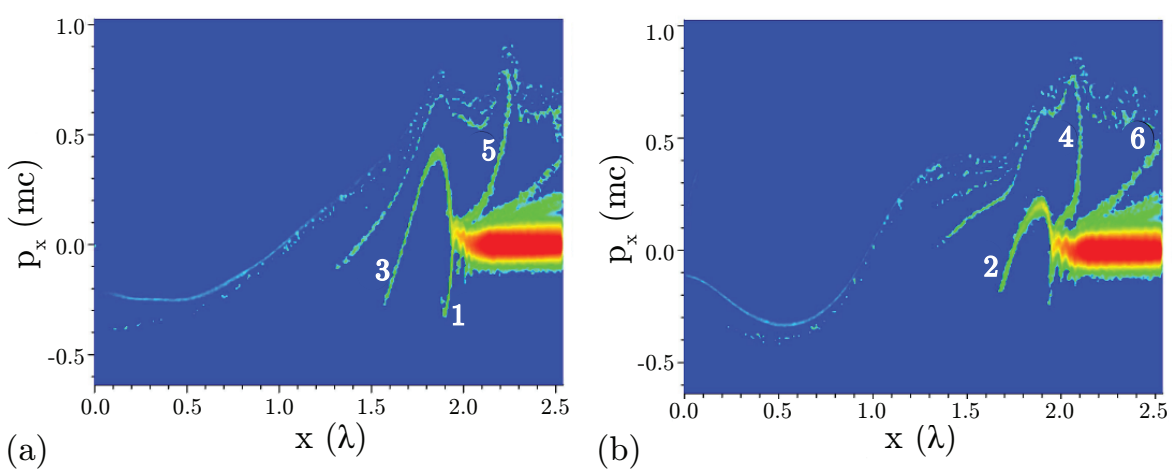

Figure 3.1: Plasma electron phase-space density for Brunel absorption, adapted from Thaury [20]. The two plots show two moments in time, where Brunel electrons were generated in different optical laser cycles. Numbers 1 to 6 indicate the different steps that the Brunel electrons follow. Step 4 corresponds to the optimum bunching in time and space.

for an ideal step-like density profile $(L / \lambda=0)$. He considered the plasma as a capacitor, where an electron sheet is driven back and forth across the target surface by the normal component of the laser electric field ${ }^{4}$. The absorption fraction derived from this approximation is given in appendix B.4 for the nonrelativistic and relativistic limit and plotted for three different laser intensities. It can be seen that in the relativistic limit there is an optimum angle predicted for the Brunel effect which occurs at approximately $70^{\circ}$.

The situation becomes more complicated for more realistic density profiles, i.e. $L / \lambda \neq 0$ [62], and the calculation of the absorption due to the Brunel effect then requires numerical modeling. The result of such calculations is illustrated in figure 3.2. The graph shows the absorption fraction originating from the Brunel effect as a function of the irradiance calculated using the equations described in appendix B.4 and as numerically calculated for finite scale lengths. It can be seen that the Brunel absorption for finite scale lengths is quite different from what Brunel derived analytically for a sharp, step-like interface. This difference is due to a highly complex transition between resonance absorption and vacuum heating that depends upon the irradiance and the scale length. For example, it can be seen that for high intensities and short scale lengths, the absorption saturates at about 10 to $15 \%$. The situation becomes even more complicated if one considers different plasma densities.

Relativistic $\mathbf{v} \times \mathbf{B}$ heating is the second absorption process that can occur at short plasma scale lengths and at relativistic laser intensities. Here, electrons

\footnotetext{
${ }^{4}$ Comparable to an oscillating mirror (see also section 3.3.1 on HOHG)
} 


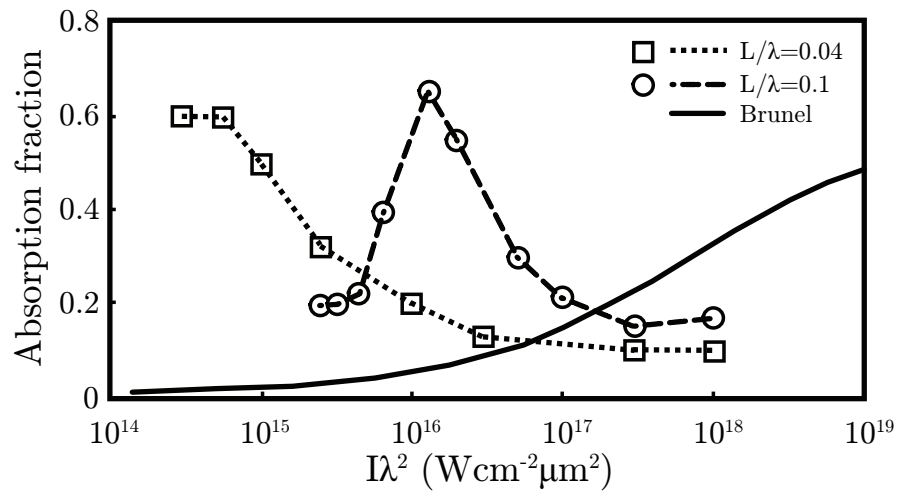

Figure 3.2: Absorption fraction versus irradiance for three different scale lengths at a 45 degree angle of incidence, adapted from Gibbon [62]. The solid line is the theoretical curve for a step-like profile according to Brunel. The dashed and dotted lines are results from numerical calculations by Gibbon and Bell for two small gradient profiles with a plasma density of $n_{e}=2 n_{c}$.

are directly accelerated by the laser field incident on a short scale-length density gradient, similar to Brunel heating. Except, with $\mathbf{v} \times \mathbf{B}$ heating, there is an additional driving term, the magnetic $\mathbf{v} \times \mathbf{B}$ component of the Lorentz force, which becomes significant at relativistic quiver velocities, and which oscillates at twice the laser frequency [62]. Due to the longitudinal direction of the Lorentz force, electrons are pushed away in the direction of the laser propagation. The number of hot electrons that are pushed away depends on the strength of the force. This absorption mechanism is most efficient at normal incidence and at low plasma densities [64]. Note, that $\mathbf{v} \times \mathbf{B}$ heating can also take place in plasma profiles with a large scale length gradient, in contrast to vacuum heating [62].

When comparing all of the named absorption and plasma processes, one finds that it is mainly the generation of hot electrons which is important for HOHG and ion acceleration $[115,120]$. This expectation is supported by the general experimental observation that the efficiency of these processes increases with the number of hot electrons and their temperature. However, different scaling laws for the temperature of hot electrons as a function of the laser irradiance have been found experimentally, ranging from $\left(I_{0} \lambda_{0}^{2}\right)^{1 / 3}$ to $\left(I_{0} \lambda_{0}^{2}\right)^{3 / 4}$. This has been attributed to the presence of different absorption mechanisms and possible transitions between them $[62,115]$. 


\subsection{Ion acceleration}

The hot electrons transfer their energy to the plasma, which can result in the generation of pulsed proton and ion beams. These beams were found to exhibit the following interesting properties: a high brightness, a low transverse divergence, a high kinetic energy and a short pulse duration. This is very promising for applications in, e.g., engineering and medicine, as mentioned in chapter 1 .

Different regimes of laser-driven ion acceleration, dependent on the laser intensity, have been identified so far. For example, by numerical modeling, rather exotic regimes are predicted at intensities above $10^{20} \mathrm{~W} / \mathrm{cm}^{2}$, such as ion shock acceleration [121,122], the so-called Laser Break-out Afterburner acceleration [123] and radiation pressure dominated acceleration [124]. However, these regimes require laser intensities higher than available with the UHI laser at PHI. These acceleration mechanisms will therefore not be discussed in further detail.

Typically, at intensities below $10^{21} \mathrm{~W} / \mathrm{cm}^{2}$ ions were found to be accelerated by electric fields that are induced by charge-separation resulting from the generation of hot electrons by the laser pulse. This is the regime that is experimentally accessible to us with the TW laser at PHI, and will be discussed in the following subsection on Target Normal Sheath Acceleration (TNSA). The advent of short-pulsed multi-terawatt lasers enabled the generation of multi$\mathrm{MeV}$ proton and ions [125] with a maximum proton energy of $58 \mathrm{MeV}$ and a maximum energy of heavy ions of $430 \mathrm{MeV}$ ( $\mathrm{Pb}$ ions) to date [126,127]. However, these energies were obtained with rather exclusive Petawatt lasers which is a fundamental problem for the general applicability of the generated beams. With TW and multi-TW lasers, i.e. with laser systems that may be developed to a much smaller size and to higher repetition rates, the maximum proton energy and the number of accelerated protons are in the order of $10 \mathrm{MeV}$, with up to $10^{9}$ particles distributed over a broad energy spectrum [115]. The highest energy protons posses the lowest divergence. A disadvantage of acceleration via the TNSA mechanism is that, without further precautions, in this very broad energy spectrum the number of protons decreases with increasing energy [128].

In order to increase the maximum energy and the number of protons with such TW lasers, we investigated the, as yet, unexplored regime of ion acceleration from special targets, namely from extremely thin foils with thicknesses as small as one nanometer. It should be noted that for many applications it is desirable to have a much narrower energy spectrum. This goal is pursued in other laboratories by, for example, using microstructured targets [23] or double-layered targets [129].

Our interest lies in investigating experimentally the applicability of ultrathin foils for ion acceleration with laser pulses with ultra-high contrast, as this has the prospect of increasing the number and maximum energy of the accelerated ions. This trend was suggested by recent experiments of others. In those experiments, foils which still had a relatively large thickness in the mi- 
cron and sub-micron range, already showed an increase of the maximum proton energy [130-133]. Also theoretically, it has been predicted that the maximum ion energy obtained with TNSA may be further enhanced if it becomes possible to work in the so-called transparent regime $[52,122]$. However, this requires foils having thicknesses in the order of the penetration depth of the laser light, i.e. in the range of a few nanometers where the preparation of suitable targets is difficult. This has previously prevented corresponding experiments. In this thesis we present the first experiments on ion acceleration in the transparent regime.

\subsubsection{Target normal sheath acceleration}

For ion acceleration, the first step is to convert a substantial part of the laser pulse energy into hot electrons. Several absorption mechanisms can play a role here (as was described in section 3.1) depending on the pulse duration and intensity and the plasma scale length. A large number of the heated electrons then move through the target and, if the target is sufficiently thin (less than typically a few micrometers), they pass through the rear surface, as is schematically depicted in figure 3.3. In part (a) of the figure it has been assumed that the pedestal of a low-contrast pulse has formed a pre-plasma with a large scale length and that, subsequently, the main pulse heats the electrons via resonance absorption or $\mathbf{v} \times \mathbf{B}$ heating. (The case of an ultra-high contrast pulse in part (b) of the figure will be considered in the following subsection.) The hot electrons that have passed through the rear surface form a sheath behind the rear-side of the target [128], which is also shown in part (a). The sheath results in a charge separation with electrostatic fields directed along the target surface normal, with a huge field strength in the order of $\mathrm{TV} / \mathrm{m}$. For comparison, the accelerating fields in conventional accelerator structures such as employed at DESY are less than $100 \mathrm{MV} / \mathrm{m}$. In the second step, this large electrostatic field accelerates protons or ions from the rear-surface into the vacuum. For instance, a contamination of the rear surface containing hydrogen, such as hydrocarbons or water, contributes to protons being accelerated. The described acceleration mechanism is called Target Normal Sheath Acceleration [128,134].

The electron sheath formed at the surface is preserved during and also well after the laser interaction, for several picoseconds, as a consequence of the plasma's tendency to maintain quasi-neutrality. When the hot electrons reach the rear surface, it is only a small fraction that escapes into the vacuum creating the electrostatic field. Electrons with energies lower than the selfinduced potential energy of the sheath, will be reflected back to the front surface. The process repeats itself at the front surface so the electrons move repeatedly back and forth through the target while they also drift transversely. An equilibrium situation sets in where the electrons that exit the foil into the sheath are compensated by electrons that re-enter the foil being reflected at the sheath [116].

The ion acceleration due to the resulting electrostatic field continues until 
the hot electrons have lost sufficient energy by adiabatic cooling or, by the retracting force of the accelerating ions, so that the strong charge separation no longer exists [135]. This happens after a few picoseconds, which is enough to accelerate the ions (mainly protons) to energies of several MeVs.

The maximum ion energy is related to the maximum electrostatic field that is formed by the electron sheath. Although the electron temperature and the plasma scale length rapidly change with time, the maximum accelerating electrostatic field can be approximately estimated by assuming that the plasma surface is initially infinitely steep, yielding [135]

$$
E=\frac{k_{B} T_{h}}{e \lambda_{D h}}=\sqrt{\frac{k_{B} T_{h} n_{h}}{\epsilon_{0}}} .
$$

The extension of the sheath is then determined by the Debye length of the hot electrons $\lambda_{D h}$ (see equation 2.1), which is typically in the order of micrometers. In the formula, $n_{h}$ and $k_{B} T_{h}$ are the density and temperature of the hot electrons. However, if there is initially a finite plasma scale length, $L$, at the surface, the density of the hot electron decreases and, if $L$ is larger than $\lambda_{D h}$, the scale length determines the distance over which the electric field acts. The accelerating electrostatic field can then be approximated by $[62,128,134,136]$

$$
E=\frac{k_{B} T_{h}}{e \max \left(L, \lambda_{D h}\right)} .
$$

By using equation 3.6, an estimation for the maximum energy of the ions accelerated by TNSA can be found $[109,115]$ :

$$
E_{\max }=2 Z k_{B} T_{h}\left[\ln \left(\tau+\sqrt{\tau^{2}+1}\right)\right]^{2},
$$

where

$$
\tau=\frac{\omega_{p i} t_{a c c}}{\sqrt{2 e^{1}}} .
$$

Here, $k_{B} T_{h}$ is temperature of the hot electrons and $t_{a c c}$ is the effective acceleration time, which scales with the pulse duration. $\omega_{p i}=\sqrt{Z n_{h} e^{2} / \epsilon_{0} m_{i}}$ is the ion plasma frequency that depends on the hot electron density $n_{h}$, which decreases with increasing scale length. Thus the maximum ion energy increases with the temperature and density of the hot electrons. Furthermore, a longer pulse results in a longer heating of the target and thus a higher number of hot electrons. This results in a higher maximum ion energy, under the condition that the rear surface of the foil remains intact during the interaction with the laser pulse. In addition, what can be seen from equations 3.6 to 3.8 , is that the maximum energy of the ions decreases when the scale length increases and when the hot electron density decreases. This can have important consequences if no care is taken to irradiate the target with pulses of sufficiently high contrast. This will be explained in the following subsection.

Equations 3.6 to 3.8 also apply to ion acceleration from the front surface. This means that when using a laser with a low pulse contrast, as shown in 
figure 3.3a, a large scale length in front of the target is formed. Consequently, the maximum energy of the ions from the front surface is small, only a few hundred $\mathrm{keV}$.

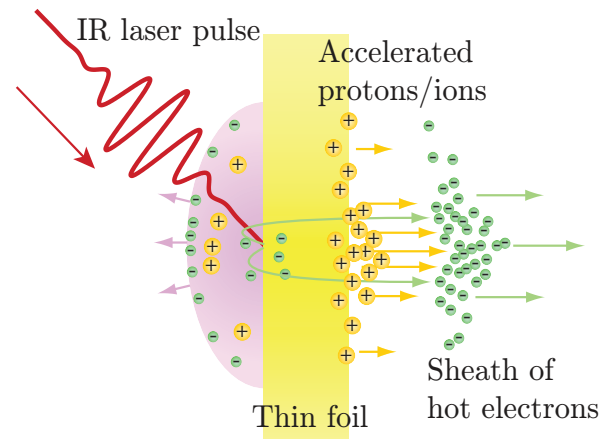

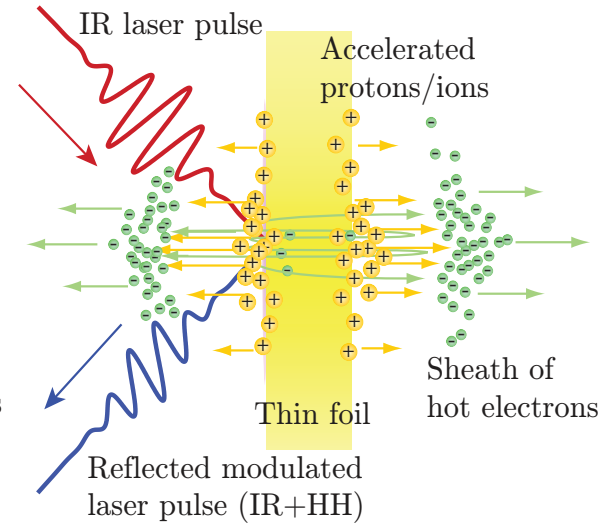

(b) By ultra-high contrast pulse

(a) Driven by low-contrast pulse

Figure 3.3: (a) Ion acceleration driven by a low-contrast (infrared) laser pulse. Ions are accelerated by a large electrostatic field generated by a sheath of laser-heated electrons at the rear surface of a foil (Target Normal Sheath Acceleration). (b) Ion acceleration driven by an ultra-high contrast pulse. In this case, the pre-plasma formed at the front surface is only small which allows ions to be accelerated in the backward direction as well. Thinner foils can be exploited to enhance the maximum ion energy. In addition, the ultra-high contrast pulse enables the generation of high harmonics $(\mathrm{HH})$ in the reflected pulse.

\subsubsection{Influence of pulse contrast and foil thickness}

Figure 3.3a shows the ion acceleration process when a low-contrast pulse interacts with a thin foil. In this case, it is the ASE of the pulse or a pre-pulse which ionizes the foil and causes a premature plasma expansion at the front surface. In addition, a shock wave is launched by the ablation pressure into the target. If the foil is too thin, this shock wave also causes the rear surface to expand before the main pulse arrives resulting in a reduced accelerating field and lower ion energies [111]. Hence, reducing the intensity of the ASE and pre-pulses, i.e. applying methods that impose a higher contrast, will prevent this effect and should allow the exploitation of the expected advantages of using thinner foils.

To increase the pulse contrast by several orders of magnitude in comparison to what is available with standard CPA laser systems, so-called plasma mirrors have recently been employed by several groups [54,57]. Using such pulses with 
ultra-high contrast and ultrathin foils as targets, increased the energy of the accelerated protons and ions emitted and, acceleration was observed on both sides of the foil $[111,130-133]$. This situation is depicted in figure 3.3b. In this condition of ultra-high contrast and high laser intensities, as described in section 3.1.3, $\mathbf{v} \times \mathbf{B}$ heating or Brunel heating are the dominant absorption processes [133,137].

The previously mentioned experiments with ultra-high contrast pulses also showed a strong dependence of the maximum proton and ion energy on the thickness of the used foils. This observation gives a promising route for improving ion acceleration, however, a clear physical picture is not available. Instead, there are several alternative suggestions, thereby leaving open the best way to more systematically exploit the potential of ultrathin foils.

A first explanation of why particles are accelerated to higher energies when using thinner foils may be that the electron density at the rear surface, and consequently the accelerating electrostatic field, is increased with thinner targets because the transverse spreading of the hot electrons inside the target is reduced [130]. As a second explanation, the electron density and temperature at the rear surface may be enhanced due to hot-electron recirculation within the target during the laser pulse $[138,139]$. Namely, for ultrathin foils, the transit time of the electrons moving back and forth is less than the laser pulse duration and the hot electrons are reflected multiple times from which they gain additional energy from the laser. Their increased thermal energy then enhances the accelerating electrostatic field, as predicted by equation 3.6. Another explanation is that the number of hot electrons and their temperature is increased by an enhanced absorption within a larger plasma scale-length due to rapid expansion [132] in combination with stronger electron confinement [133].

What has been found in experiments, when decreasing the foil thickness, is that there is a certain optimum thickness below which the maximum particle energy decreases again, and that this optimum thickness shifts towards lower values when the pulse contrast is increased. A possible explanation for this is that at the optimum thickness for a high-contrast pulse, a lower-contrast pulse causes a perturbation at the rear surface of the foil which, consequently, decreases the accelerating field $[130,131]$.

Yet two other differences between low-contrast and high-contrast became noticeable in particle acceleration from thin foils. Firstly, with a low-contrast pulse, the kinetic energy of ions found in the backward direction (i.e., from the front surface of the foil in the opposite direction of the laser beam), yields a lower maximum energy compared to the energy in the forward direction (from the rear surface of the foil). This was attributed to the expansion of the plasma towards a larger scale length at the front surface [140]. For highcontrast pulses, on the other hand, the acceleration seems to be comparable in both directions $[133,141]$. Secondly, with high-contrast pulses, the acceleration is clearly related to the strength of only the p-component of the laser's electric field and is not effective for s-polarized light, which points to Brunel heating as the underlying mechanism [133]. In the low-contrast regime, it was observed 
that both polarizations contribute to ion acceleration, which would point to heating via the $\mathbf{v} \times \mathbf{B}$ mechanism [142].

\subsubsection{Ion acceleration in the transparent regime}

Numerical calculations predict the existence of an especially promising regime for ion acceleration, however, this is difficult to reach. In this so-called transparent regime of ion acceleration $[52,122]$, the basic mechanism is similar to TNSA but the number of hot electrons and their energy are increased. Accordingly the accelerating field, described by equation 3.6, increases and so does the maximum kinetic energy of the ions. When going toward thinner foils, the optimum foil thickness for the maximum ion energy is found at a thickness just below the onset of the opaque regime. The maximum energy shows a strong decay beyond the optimum thickness and a weaker decay in the opaque regime. At the optimum thickness, in the transparent regime, the maximum energy of the accelerated ions is twice as high as the maximum energy at the transition to the opaque regime. An example of such a prediction is given in figure 5.19 which shows the results of our numerical calculations described in section 5.4, and is in agreement with the previous calculations of others [52,122].

The numerical calculations $[52,122]$ show that in this transparent regime, the optimum foil thickness depends linearly on the laser intensity and the pulse duration and is inversely proportional to the plasma density. The electrons stay well-confined at the beginning of the interaction. However, they obtain a small additional transverse momentum from the laser pulse as it passes through the plasma slab to the rear surface. As a consequence, the protons also obtain a larger divergence compared to ion accelerated in the opaque regime $[52,122]$.

So far, the theoretical predictions on ion acceleration in the transparent regime have not been verified experimentally for two reasons. First, the high plasma density of solid foils requires the use of extremely thin foils with thicknesses of less than $10 \mathrm{~nm}$, the preparation and use of which is experimentally difficult. So far, the thinnest foil investigated for ion acceleration is $20 \mathrm{~nm}$ thick [131]. A second limiting factor is the ultra-high contrast of the pulses needed. With an insufficient pulse contrast it was found that the maximum proton energy decreases significantly for foil thicknesses below approximately $100 \mathrm{~nm}$. In chapter 5, we will present the first experimental results on TNSA in the transparent regime, and discuss the requirements for the experimental conditions, such as the laser pulse contrast and the foil properties.

\subsection{High-order harmonic generation}

In addition to ion acceleration, the generation of hot electrons via the Brunel effect or relativistic $\mathbf{v} \times \mathbf{B}$ heating (during high-intensity laser-solid interactions) is also the underlying physics for the generation of high-order harmonics from solids (HOHG). Therefore, when utilizing laser pulses with an ultra-high 
contrast, ion acceleration and HOHG may simultaneously appear during interaction with a solid target. This is illustrated in figure 3.3b. High-order harmonic generation from solids is of significant interest and has huge future potential because it has no limitation in the intensity. Compared to high harmonic generation in bound electron systems (HHG), such as neutral gases or partially ionized gases (ions) where the shortest generated wavelength is limited by the requirement of binding to a nucleus, HOHG from solids is free of such limits because it makes use of a fully-ionized plasma, which means that in principle HOHG has no cut-off. The strong interest in HOHG was initiated by theoretical analysis, such as its description via a relativistic oscillating plasma mirror [143-146]. To provide a better understanding of the HOHG process and the properties of the high-order harmonics generated via this process, a more detailed description of the Relativistic Oscillating Mirror (ROM) model will be presented below. Early experiments performed in the relativistic regime showed high harmonics up to the $75^{\text {th }}$ order [147-154]. Recently, harmonic radiation up to the $3200^{\text {th }}$ order $(3.8 \mathrm{keV})$ has been generated using laser intensities of more than $10^{20} \mathrm{~W} / \mathrm{cm}^{2}[155,156]$, proving the high potential of HOHG.

Complementary to the ROM model, high-order harmonics can be generated at non-relativistic intensities via another mechanism, which explains the results of early experiments where only a few harmonic orders were obtained with frequencies below the plasma frequency [157-161]. The model that describes this mechanism is called Coherent Wake Emission (CWE) [120] and will be presented in more detail in subsection 3.3.2. High-order harmonics that are generated via this mechanism possess different properties than high-order harmonics from ROM. There exists a transition regime where both mechanisms can contribute to the generation of high-order harmonics with comparable strength $[20,162]$, which will be described at the end of this chapter. This transition regime is reached in our experiments at intensities around $10^{19} \mathrm{~W} / \mathrm{cm}^{2}$, which will be discussed in chapter 5 . The different properties of the high-order harmonics generated via both processes allow to experimentally distinguish between harmonics generated by each of the two mechanisms.

\subsubsection{Relativistic Oscillating Mirror}

High-order harmonics generation (HOHG) by the ROM mechanism has the highest prospects for future bright, coherent X-ray sources. The advantage of HOHG via ROM is that the harmonic spectra can be extended far into the regime of hard X-rays because there is no principle limit on the intensity. Also, the radiation is highly coherent in time and space, in particular, the radiation is Fourier-limited and diffraction-limited with respect to the divergence of the drive laser $^{5}[156,163]$.

High-order harmonics generated via ROM is, as the name indicates, caused

\footnotetext{
${ }^{5}$ Under perfect conditions (spatially constant intensity of the drive laser and a flat and smooth surface) the diffraction-limited divergence of the $n^{\text {th }}$ harmonic should equal $\theta_{n}=$ $\theta_{0} / n$, where $\theta_{0}$ is the divergence of the drive laser beam [163].
} 
by reflection of a laser pulse from an oscillating, mirror-like plasma surface [143-145]. This situation is depicted in figure 3.4. It is considered that this oscillation of the effectively reflective plasma surface, $x_{o s c}(t)$, is driven by the incident high-intensity IR laser field. Then, the reflection of the IR pulse (by the self-driven mirror) modifies the laser waveform and hence produces highorder harmonics (see the deformations in the reflected pulse in figure 3.4a), while the coherence of the wave is preserved. Qualitatively, the generation of harmonics can be understood as caused by the Doppler effect when light reflects on a moving mirror [164], schematically shown in figure 3.4b. As an example, if the mirror would move with a constant, but high, velocity, $v$, close to the speed of light (constant Lorentz factor $\gamma$ ), the entire spectrum of the incident pulse would become frequency up-shifted by a significant constant factor (by $4 \gamma^{2}$ ) and thus compressed in time by the same factor ${ }^{6}$, where $\gamma=\left(1-v^{2} / c^{2}\right)^{-1 / 2}$. Thus the more the mirror velocity approaches the speed of light, the higher the frequency up-shift becomes and the stronger the incident IR pulse becomes compressed.

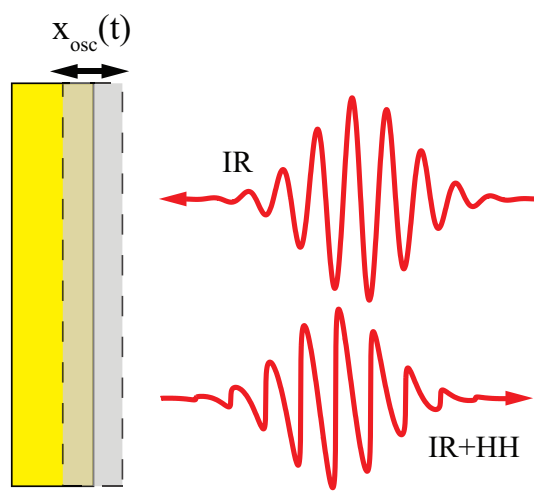

(a) Relativistic oscillating mirror

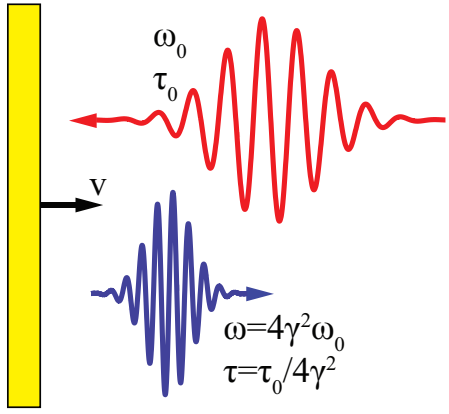

(b) Relativistic Doppler upshift

Figure 3.4: Frequency up-shift by reflection off (a) a relativistic oscillating mirror (ROM) and, (b) a relativistic mirror moving with a constant velocity. With ROM, frequency up-shift only occurs when the mirror, formed by plasma electrons (gray), moves towards the laser pulse. The pulse is strongly and periodically modified which leads to the generation of high-order harmonics $(\mathrm{HH})$. With a mirror moving with a constant velocity, the complete pulse is up-shifted and compressed in time.

In an experiment of $\mathrm{HOHG}$ with a periodically oscillating mirror, as illustrated in figure $3.4 \mathrm{a}$, the Lorentz factor associated with the mirror velocity is not constant [156]. Instead the Lorentz factor oscillates periodically with the

\footnotetext{
${ }^{6}$ More exactly: $\omega_{\text {Doppler }}=\omega_{0} \cdot(1+v / c) /(1-v / c)[164]$
} 
electric field of the laser that drives the mirror motion by pulling out electrons into the vacuum and pushing them back into the plasma, via the Brunel effect or $\mathbf{v} \times \mathbf{B}$ heating. As a result, the reflected light wave is periodically compressed and its phase is modulated non-sinusoidally. A Fourier analysis of the modulated light then shows harmonics of the incident laser frequency. Particularly, in the ultra-relativistic limit $\left(a_{0} \gg 1\right.$, where $a_{0}$ is defined by equation 2.12), the Lorentz factor of the oscillating surface shows sharp spikes in time (so-called $\gamma$-spikes), which are much shorter than the optical cycle of the laser [165]. (The number of $\gamma$-spikes per optical cycle depends on the incident laser polarization and the corresponding absorption mechanism, which will be discussed below.) Then, not only the height of the $\gamma$-spikes, $\gamma_{\max }$, increases with $a_{0}$, but also the temporal duration of the spikes reduces, inversely with increasing $\gamma_{\max } \cdot{ }^{7}$ Then the emission of high-order harmonics becomes limited to an extremely short time duration, thereby generating extremely short pulses of harmonic radiation. Taking into account the Doppler effect upon reflection, the high-order harmonic radiation becomes compressed by a factor $4 \gamma_{\max }^{2}$ and is therefore emitted in short temporal bursts with a duration of $T_{\text {burst }} \propto T_{\gamma-\text { spike }} / \gamma_{\max }^{2} \propto T_{0} / \gamma_{\max }^{3}$, with $T_{0}$ being the optical cycle of the incident laser pulse [166]. These relationships show that when realizing a sufficiently high $\gamma_{\max }$, the process can easily result in the generation of a train of attosecond pulses, the optical spectrum of which contains a large number of harmonic orders. In principle it should even be possible to obtain a train of zeptosecond pulses. These may best become visible if the lower spectral components are filtered out of the reflected pulse [167].

The short duration of the harmonic bursts also becomes apparent from a Fourier analysis of the spectrum of the harmonic radiation, where the maximum frequency scales with $\gamma_{\max }^{3}$. It was shown theoretically that the conversion efficiency of the $q^{\text {th }}$ harmonic order scales as

$$
\eta(q) \propto q^{-8 / 3},
$$

up to a roll-over order of

$$
q_{R O} \propto \sqrt{8} \gamma_{\max }^{3}
$$

beyond which the conversion efficiency decreases more rapidly [165]. Because $\gamma_{\max }$ increases with increasing laser intensity, the advantage of HOHG by ROM is that the harmonic spectra can be extended far into the regime of hard X-rays since there is no principle limit on the intensity. Recently, the power scaling and harmonic roll-over of the $\gamma$-spike theory were experimentally confirmed by the generation of X-ray harmonic radiation extending to the $3200^{\text {th }}$ order $(3.8 \mathrm{keV})$ with a fundamental laser wavelength of $1053 \mathrm{~nm}[155,156,166]$. The experiments also yielded diffraction-limited radiation, based on the divergence of the incident laser beam and the curvature of the plasma surface. This is a strong indication of the coherent nature of the harmonic generation process $[156,163]$.

\footnotetext{
${ }^{7} \gamma_{\max }$ corresponds to the maximum surface velocity and is calculated by $\left(1+a_{0}^{2} / 2\right)^{1 / 2}$.
} 
In experiments, different configurations can be used with respect to the angle of incidence and the polarization of the drive laser, namely normal incidence and oblique p- or s-polarized light. The harmonic spectrum and the polarization properties of the harmonic beam depends on these parameters, which are summarized in table 3.1 [144]. This is related to the underlying absorption mechanism, as follows. The oscillating surface required for HOHG is to be realized as a collective oscillation of a dense bunch of plasma electrons. Physically, such a bunch of electrons can be brought into oscillation via two mechanisms. Depending on the laser polarization and angle of incidence, the electrons are repeatedly pulled into the vacuum and pushed back by Brunel absorption or $\mathbf{v} \times \mathbf{B}$ heating (see section 3.1.3). For example, a linearly polarized pulse at normal incidence to the plasma surface results in only odd harmonics. This is because the electrons are dragged out of the plasma twice per optical cycle by the ponderomotive part of the Lorentz force $(\mathbf{v} \times \mathbf{B}$ heating mechanism). Therefore, only odd harmonics are generated. To compare this with p-polarized light, Brunel electrons are pulled out by the laser field once each optical cycle such that also even harmonics are produced. It turned out that obliquely s-polarized light results in less efficient harmonic generation due to the less efficient heating of the plasma electrons.

\begin{tabular}{lcc}
\hline Incident pulse & Odd harmonics & Even harmonics \\
\hline Oblique $\mathrm{s}$ & $\mathrm{s}$ & $\mathrm{p}$ \\
Oblique $\mathrm{p}$ & $\mathrm{p}$ & $\mathrm{p}$ \\
Oblique circular & $\mathrm{s}, \mathrm{p}$ & $\mathrm{s}, \mathrm{p}$ \\
Normal linear & linear & - \\
Normal circular & - & - \\
\hline
\end{tabular}

Table 3.1: Harmonic selection rules

The process of HOHG is, as a physical model, rather straight forward to understand, however, its realization in an experiment puts strict conditions on the laser and the target due to the following reasons. A high efficiency and fidelity of the reflection off a plasma mirror requires a high-quality (ideally flat and sharp) plasma-vacuum transition in order to have a well-defined reflection point and prevent destructive interference from waves reflected at different positions [156]. This also ensures Fourier-limited [168] and diffraction-limited harmonic pulses [163]. Accordingly, for a modeling of ROM a step-like profile is often used $[144,165,169]$. In an experiment, however, a flat and step-like plasma-vacuum interface, driven to relativistic oscillation by the laser field, is difficult to realize. For example, the local oscillation amplitude of the plasma electrons grows with the local strength of the laser field and decreases with the plasma restoring force, and the latter grows linearly with the plasma density. This means that increasing the density of the plasma, in order to increase the amount of reflected harmonic power, simultaneously reduces the electron excur- 
sion thereby rendering less power at the higher orders of the spectrum. Indeed, numerical calculations where a step-like profile is assumed, show that low densities (several times the critical density), result in a higher harmonic output. However, such low density plasma profiles are experimentally not achievable because the density of solid targets is very high (several hundreds times the critical density $n_{c}$ ). Numerical calculations of ROM with a high plasma density, show that HOHG still can be efficiently driven when a short scale length gradient is assumed. The laser then interacts with the low-density region around the critical surface with weaker restoring forces [162]. It was found, both in numerical modeling and in experiments, that there exists an optimum value for the scale length of around $L / \lambda \approx 0.2[149,156,162]$. A plasma profile with a larger scale length deviates from a mirror-like surface and suppresses the harmonic output [156]. Furthermore, a large pre-plasma is associated with plasma deformations and an increase of the divergence of the harmonics [110]. It should be noted that to obtain such a short plasma gradient in experiments, laser pulses with an ultra-high contrast have to be used since a short plasma scale length is increasingly difficult to achieve with increasing intensities.

\subsubsection{Coherent Wake Emission}

Experiments have shown that even when the applied laser intensities are too low to induce the previously described high-order harmonic generation via an oscillating mirror, in the range of $10^{16}$ to $10^{17} \mathrm{~W} / \mathrm{cm}^{2}$, high harmonics were still generated. A mechanism that explains this observation is generation via coherent wake emission (CWE) [20,120], which is schematically depicted in figure 3.5. In general, CWE relies on the generation of short, energetic electron bunches that are driven into a small plasma gradient $(L / \lambda<0.1)$ where they excite high-frequency plasma oscillations in their wake. These plasma oscillations subsequently radiate light that emerges in the direction of the reflected laser beam [120,162]. This process occurs every optical cycle and thus the radiation consists of harmonics of the drive laser frequency, with a maximum frequency that is equal to the maximum plasma frequency of the target.

In more detail, the first step of the process (see figure 3.5a, taken from Thaury et al. [20]) is that the component of the laser field normal to the target pulls out electrons, as described in section 3.1.3 on Brunel absorption. As the field decreases and changes sign, electrons are pushed back into the plasma which is also supported by space-charge electrostatic fields (figure 3.5b,c). An attosecond electron bunch is formed because the returning Brunel electrons overtake each other in a synchronized manner: electrons that had moved further into the vacuum come back into the plasma with larger velocities than electrons that had smaller excursions (as was also shown in figure 3.1). An oblique density front of electron bunches (yellow thick line in figure 3.5c) propagates through the plasma because of the obliquely incident laser pulse. As this wavefront propagates through the plasma gradient, a plasma wave is induced in its wake due to the collective behavior of the plasma (figure 3.5c), as 


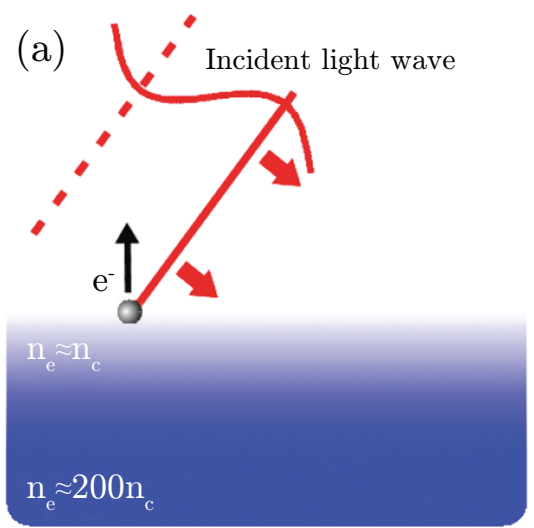

(c)

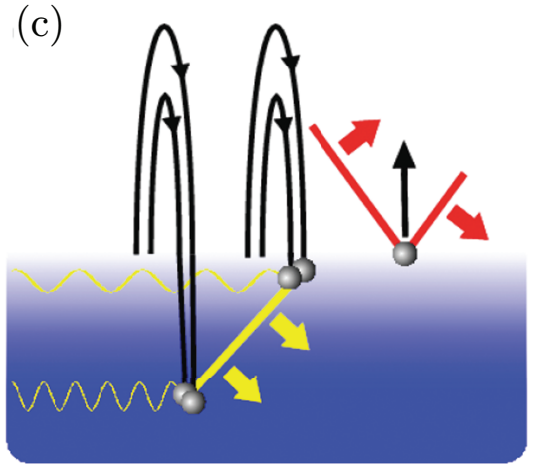

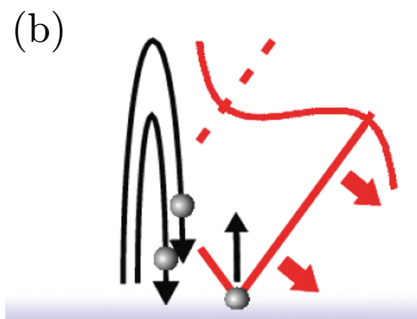

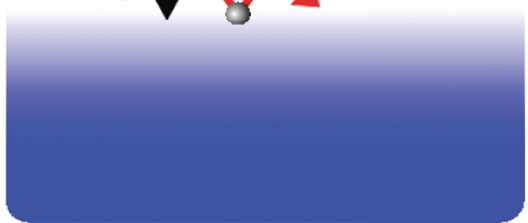

(d)

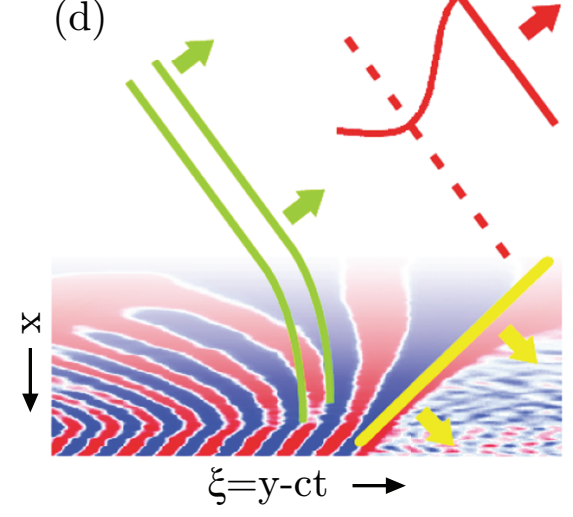

Figure 3.5: Different stages of CWE, as adapted from Thaury et al. [20]. The figure shows a plasma with a density gradient where a light wave (red front) is incident under an angle. (a) The electric field pulls electrons out of the plasma around the critical density and then (b) pushes them back into the plasma with high energy (the Brunel effect). (c) The electrons propagate into the overdense plasma unhindered by the screened laser field where they form an attosecond bunch. The oblique density front (thick yellow line) excites plasma oscillations in its wake (yellow sinusoids) of which the wavelength becomes shorter with increasing density. (d) shows the wavefronts (red and blue) of the plasma oscillations, obtained by Quéré et al. [120] from numerical calculations with a particle-in-cell code. These wavefronts are curved in space, or alternatively, make a curvature in time (via the transformation $\xi=y-c t)$. The plasma oscillations emit an attosecond light burst (green lines) when the k-vector of their wavefronts is parallel to the surface of the plasma. 
described in section 2.1. Figure 3.5d shows the wavefronts (red and blue) of the plasma oscillations, obtained from numerical calculations with a particle-in-cell code by Quéré et al. [120]. These wavefronts are curved in space, which can be seen as a gradual rotation in time, when looking at a fixed position, via the transformation $\xi=y-c t$.

The second step in the process is the emission of radiation, which is schematically illustrated in figure $3.5 \mathrm{~d}$. In a similar way that an obliquely incident electromagnetic wave can be resonantly absorbed and transfer its electromagnetic energy to plasma waves in an inhomogeneous plasma (see section 3.1.3 on resonance absorption), here the inverse process occurs in which the kinetic energy and potential energy contained in the plasma oscillations is radiated into transverse electromagnetic waves, such that the generated electromagnetic frequencies are equal to the plasma frequencies. This process is also referred to as linear mode conversion [170]. Light can be emitted when the wave vector of the plasma wave has a transverse component (parallel to the surface). However, plasma oscillations and electromagnetic waves possess a different dispersion relation. Therefore, phase-matching between both waves is required for efficient mode conversion. According to calculations by Sheng et al. [171], this only occurs when the wave vector of the plasma wave is parallel to the plasma-vacuum interface.

Phase-matching is intrinsically fulfilled in CWE, which can qualitatively be understood as follows. Due to the density gradient, the plasma oscillations have a space-dependent frequency. Furthermore, the plasma oscillations with shorter wavelengths (higher frequencies) are excited at later times. As a result, the plasma wave front gradually rotates, as is visible in the PIC calculations shown in figure 3.5d [120]. This means that during only a fraction of an optical cycle (a few hundred attosecond), the wave vector of the plasma oscillations is parallel to the plasma surface which is the necessary condition for phasematching. Consequently, a train of attosecond pulses is emitted. The pulses are positively chirped because higher frequency radiation is generated in deeper regions of the plasma at later times and propagates longer through the plasma. The emitted radiation propagates and refracts through the plasma and subsequently emerges under an angle that equals the angle of reflection of the laser pulse [20]. Because of the repetition with every optical cycle the spectrum of the radiation consists of high harmonics of the incident laser pulse with a maximum frequency that equals the maximum plasma density. The efficiency for the $10^{\text {th }}$ harmonic order (as an example) was derived from numerical calculations to be $10^{3}$ to $10^{4}$, but has not yet been quantitatively determined in experiments.

In order to compare the properties of the high-order harmonics due to CWE with the properties of the harmonics due to HOHG via ROM, to discriminate experimentally the harmonics generated by both processes, the coherence properties of CWE are discussed in more detail in the following paragraphs.

The individual attosecond pulses (that consist of many harmonic orders) are 
positively chirp, as mentioned above. This means that lower order harmonics (lower frequencies) are emitted before the higher orders (higher frequencies). To the contrary, the individual harmonic orders possess a negative chirp (blue precedes red), which qualitatively means that the emission time between successive attosecond pulses in the train increases, due to the changing intensity envelope of the incident laser pulse and an evolving plasma gradient. This can be understood as follows. An illustrative example is given in appendix C. The peak velocity of the Brunel electrons scales with the intensity. As a consequence, the moment when the plasma oscillations are induced is also intensity dependent and hence the time of emission of the attosecond pulses (and thus the phase of the high-order harmonics) varies with the intensity. In addition, the time of emission is at a later moment for an increasing plasma density scale length. The combination of the changing intensity envelope of the incident laser pulse and the increasing plasma gradient leads to an increasing time spacing in between successive attosecond pulses, which via Fourier analysis results in a negative chirp and broadening of the individual harmonic orders $[120,168]$, similar to the harmonic chirp found in HHG from gases (see also appendix C).

The intensity-dependent phase variation of the harmonics also has consequences for the spatial properties of the high-order harmonics. Because of the intensity variation in the spatial envelope of the incident laser pulse, the harmonic beam is emitted with a curved spatial wave front, which shows, in the far-field, as an increased divergence. To compare this with ROM, the relativistic Doppler reflection of the IR laser into harmonics occurs always at the same moment during an optical cycle, independent of the laser intensity, which leads to a harmonic beam that is diffraction-limited with respect to the divergence of the incident drive laser.

It is important to note that high harmonics due to CWE have been generated from the front and rear surfaces of ultra-thin foils with thicknesses of 50 to $400 \mathrm{~nm}[160,161]$. This is important as it applies to our experiments with nanofoils. The harmonics from the rear surface are due to electrons that penetrate through the foil and return (re-circulate), and induce plasma oscillations in the rear side density gradient that subsequently emit CWE harmonic radiation.

To summarize, current understanding of harmonic generation from solids is based on two different mechanisms, ROM and CWE, each with a distinct set of properties of the high-order harmonics. An essential difference between the two is that harmonics via ROM are generated on the surface of the overdense plasma, whereas harmonics via CWE are generated in deeper regions of the plasma a fraction of an optical cycle later by plasma oscillations induced by Brunel electrons. Furthermore, CWE can occur at non-relativistic laser intensities, while ROM requires relativistic intensities. There also exists a transition regime where both mechanisms of HOHG can simultaneously occur. This is illustrated in figure 3.6, which shows the result of numerical calculations made by the PHI group at Saclay [120]. The figure shows a plot of the electron den- 
sity of a plasma foil $\left(n_{\max }=80 n_{c}\right)$ with initially a small gradient $(L / \lambda=1 / 15)$ along the target normal (directed along $x$ ) as a function of time. Also plotted is the envelope of the magnetic field $B_{z}$ of the harmonics 5 to 9 (purple). A laser pulse with a constant intensity $\left(6.3 \cdot 10^{17} \mathrm{~W} / \mathrm{cm}^{2}\right)$ is incident from the top at an angle of incident of $45^{\circ}$ (propagates from negative to positive $x$ ). The white arrows indicate the time when a dense bunch of Brunel electrons has penetrated the plasma and exit at the rear side. Two types of separated attosecond pulses can be seen every optical cycle. One of these pulses comes from the density surface and can be attributed to the ROM mechanism. The second pulse is generated a fraction of an optical cycle later, in deeper regions of the plasma, and can be attributed to the CWE mechanism.

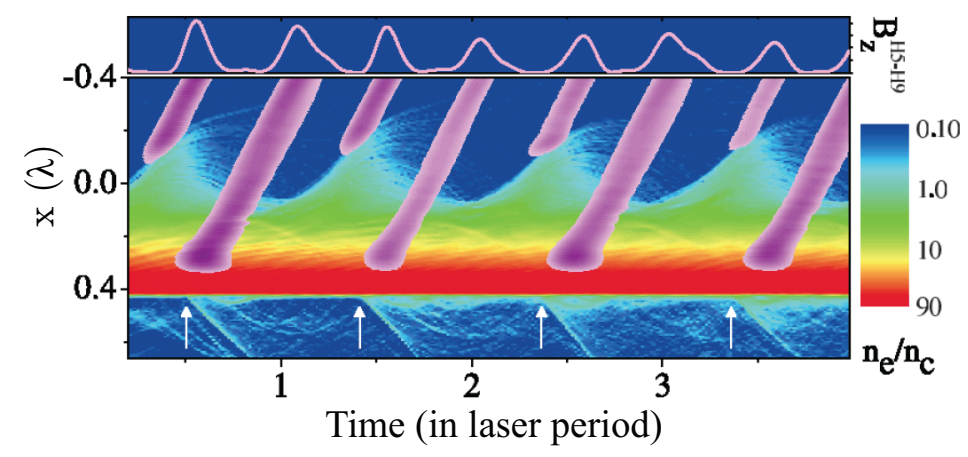

Figure 3.6: Results from PIC calculations made by Quéré et al. [120]. Calculated electron density of a plasma slab in log scale and the intensity of the generated harmonic pulses (purple color scale) are shown in time and space during the interaction with a highintensity laser pulse incident from the top (not visible). Each optical cycle there are two harmonic pulses generated: at the surface by ROM and inside the plasma by CWE. The upper panel shows the temporal profile of the attosecond pulses at $x=-0.4 \lambda$. The white arrows indicate the time when a dense bunch of Brunel electrons penetrates the plasma and exit at the rear side.

In order to experimentally distinguish harmonics generated by both mechanisms in such a transition regime, one can exploit the different properties of the high-order harmonics. These properties are briefly summarized in table 3.2. The maximum harmonic frequency by CWE depends on the plasma density, whereas the laser intensity is the limiting parameter with ROM. An essential difference between the two processes is that the phase of harmonic emission is intensity-dependent with CWE. On the contrary, with ROM all harmonic frequencies are generated at the same moment in time, independent of the incident laser intensity. This means that the harmonic pulses generated by ROM are Fourier-limited and diffraction limited with respect to the divergence of the 


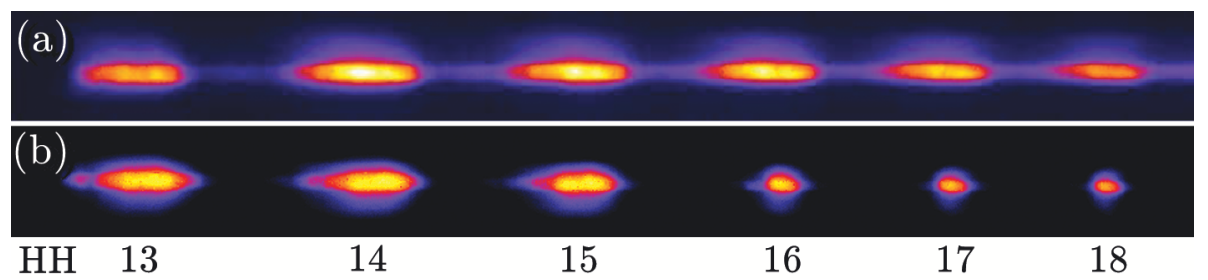

Figure 3.7: Raw images of HOHG spectra on the detector, adapted from Thaury [20], obtained from (a) a silica target at $3 \cdot 10^{18} \mathrm{~W} / \mathrm{cm}^{2}$ and (b) a plastic target at $8 \cdot 10^{18} \mathrm{~W} / \mathrm{cm}^{2}$. Compared to silica, the plastic target has a lower maximum plasma density, which corresponds to a maximum harmonic order due to CWE of 15. The difference in spectral width between harmonics due to CWE and ROM beyond the 15th order is striking.

drive laser, as opposed to the chirped harmonic pulses from CWE which posses a slightly larger divergence. The chirp can be distinguished between a chirp of the individual attosecond pulses and a chirp of the individual harmonic orders.

These differences become visible in the spectrum, as is illustrated in figure $3.7[20]$. Figure 3.7a shows a recorded spectrum from silica at an intensity of $3 \cdot 10^{18} \mathrm{~W} / \mathrm{cm}^{2}$ where only harmonics due to CWE have been generated. Figure $3.7 \mathrm{~b}$ shows a spectrum from plastic at an intensity of $8 \cdot 10^{18} \mathrm{~W} / \mathrm{cm}^{2}$. Here, harmonics via CWE are generated only up to the $15^{\text {th }}$ order, which is due to the lower density of plastic. Harmonics above this order are generated via ROM. These harmonics due to ROM are much narrower compared to harmonics via CWE, which is the result of the differences in the chirp.

\begin{tabular}{ll}
\hline ROM & CWE \\
\hline$\omega_{R O}=\sqrt{8} \gamma_{\max }^{3} \omega_{0}$ & $\omega_{\max }=\omega_{\text {p,max }}$ \\
$\eta \propto q^{-8 / 3}$ & $\eta \approx 10^{-3}-10^{-4}$ \\
Fourier-limited attosecond pulses & Positively chirped attosecond pulses \\
Narrow spectral harmonic orders & Negatively chirped and broadened \\
& harmonic orders \\
Diffraction-limited emission & Low divergent beam \\
\hline
\end{tabular}

Table 3.2: Properties of high-order harmonics due to ROM and CWE. Note that the efficiency for CWE is derived from numerical calculations and has not yet been quantitatively confirmed in experiments. 


\subsection{Discussion}

The interaction of high-intensity lasers with overdense plasmas is highly complex due to light-induced, ultrafast and local changes of the plasma density and, the strong back-action of these onto the light field distribution. Different types of absorption mechanisms can be responsible for energy transfer from the laser to the plasma, depending on the laser intensities and plasma gradients. These mechanisms, and associated heating of the plasma, can result in the generation of hot electrons which can lead to the generation of high-order harmonics and the acceleration of ions. Due to the basic interconnection of their dynamics, both these physical processes are very dependent on the contrast of the drive laser pulse. In particular, a laser with an ultra-high contrast and that is simultaneously capable of generating the highest intensities towards the relativistic regime is required for optimum output in both processes. Such lasers are at the forefront of current laser technology, and a laser with record breaking specifications is available at the PHI group at CEA Saclay in France.

Increasing the laser pulse contrast lowers the minimum foil thickness that can be used for ion acceleration. This is important because the number and maximum energy of ions increases with a decreasing foil thickness, down to an optimum thickness. Theoretically, with an infinite pulse contrast, the optimum foil thickness is what brings the plasma into the transparent regime. This requires the use of foils with a thickness in the order of the penetration depth of light in overdense plasma, which is only a few nanometers for solid density targets. We prepared such extremely thin foils on special target holders in order to enable the first experimental investigation of ion acceleration in this transparent regime. These investigations and the corresponding results are described in chapter 5 .

Similarly, one could expect an enhancement in HOHG output when utilizing extremely thin foils, which can be seen as follows. Both coherent wake emission $(\mathrm{CWE})$ and the relativistic oscillating mirror $(\mathrm{ROM})$ require a short plasma scale length for an optimum output which means that laser pulses with an ultra-high contrast have to be used. For high-order harmonic generation by CWE, a small density gradient is needed to sustain plasma oscillations driven by Brunel electrons, but that allows the transmission of the generated light by these plasma oscillations. Tarasevitch found in numerical calculations that the harmonic output is suppressed for a scale length larger than $L / \lambda \approx 0.1$ [162]. For HOHG via ROM, it is desired to have a mirror-like low-density overdense plasma (slightly above the critical density) to induce large electron excursions for efficient harmonic conversion. However, due to the large density of solid targets, and as a result the strong plasma restoring force, the excursion of electrons into the vacuum is limited for step-like density profiles. Therefore, with such high plasma densities there exists an optimum scale length for the plasma density gradient, which is in the order of $L / \lambda<0.2$. For an optimization of $\mathrm{HOHG}$ one could try to reduce the undesired plasma restoring force by reducing the thickness of the involved plasma slab into the ultra-thin range by 
decreasing the thickness of the foils into the nanometer range as well.

Based on these considerations, we decided to simultaneously perform measurements of HOHG and ion acceleration from nanofoils which will be discussed in chapter 5. In the past, foils with thicknesses larger than $40 \mathrm{~nm}$ have been used for HOHG, however, the harmonic signal was then found to be due to CWE and not to ROM. In our experiments we use thinner foils and higher laser intensities. Nevertheless, although we successfully generated high harmonics in this unexplored regime (from foils as thin as $7 \mathrm{~nm}$ ) the experimental results did not allow the confirmation of such an enhancement of HOHG. In order to investigate a possible enhancement of HOHG from extremely thin foils, we performed numerical calculations, which also will be discussed in chapter 5 .

In addition to the experiments on HOHG and ion acceleration from extremely thin foils, we also performed an experiment on HOHG from bulk solid targets in the regime of CWE. Although the harmonic radiation is expected to be coherent, this had not yet been proven experimentally. We performed such experiments by recording the interference pattern of mutually coherent sources of CWE. In addition, because the emission of harmonic radiation in the case of CWE depends on the intensity of the drive laser, these interference patterns contain information about the dynamics of plasma electrons. This is further exploited in experiments described in chapter 5 . 
key to success.

Alexander Graham Bell (1847 - 1922)

\section{Generating high harmonics in gas-filled capillaries}

The front-end of the laser system in assembly at LPNO started to deliver, less than two years ago, ultrashort pulses with energies that should allow the experimental investigation of high harmonic generation in gaseous media (HHG). We decided to devise and construct a setup to perform such experiments. As the goal of this activity, we decided to make use of the existing expertise on plasma, discharge techniques and laser-based UV generation to explore novel approaches that enhance the harmonic efficiency and the cut-off frequency. As a particularly interesting route and for its great potential, we identified harmonic excitation (HEx) in mixtures of gases or partially ionized gases (ions), especially, in combination with QPM techniques, as discussed in section 2.3. The eventual goal is to open up research in a novel field, namely nonlinear optics in the XUV regime, where the coherent XUV radiation obtained with HHG is focused with special XUV optics. This should enable ultra-small spot sizes, in the nanometer or sub-nanometer range, associated with enormous intensities, beyond that of the drive laser.

Due to the high level of technology involved in such a framework, starting research in this direction requires progress to be taken over several steps. The first step was to realize and test an adequate functioning experimental apparatus. Our goal, over a two year period, was to design and construct the setup and perform the first test measurements of HHG. In the process we would also gain the expertise in the field of HHG necessary for a proper approach in subsequent steps. Indeed, the construction of the experimental setup for HHG resulted in a working apparatus which could be operated in time-sharing with the further progressing upgrading of the laser system for experiments on laser 
wakefield acceleration.

This chapter describes the experimental considerations for the setup and the diagnostics in section 4.1, in which we start with a description of the parameters of the laser pulses that have been used for generating high harmonics. In order to optimize the performance of HHG, during the construction phase and test measurements, the laser, particularly, the stretcher and compressor had to be optimized. Modifications to the laser were realized in steps to avoid delay of the LWFA experiments, for example, full optimization of the stretcher was performed only very recently. As a result, most of the test measurements presented here are based on pulses with a somewhat extended duration and an elliptical beam cross section.

In this section, the advantages and potential improvements of the setup will be discussed. The current setup makes use of a capillary waveguide. A capillary waveguide was chosen as previous research had reported a higher harmonic output and a higher spatial coherence when compared to a gas jet, as was discussed in chapter 2. A second advantage of using a capillary is that this, subsequently, enables a relatively straightforward extension towards HHG in ions, in a plasma waveguide (capillary discharge).

The capillary design was a main point of concern in the setup design. Ideally, the pressure of the gas in the capillary should remain constant over the interaction length with the laser. However, this is difficult to achieve experimentally due to the necessary transition to the vacuum at the end of the capillary. In order to approximate the desired situation as close as possible, we implemented the well-known technique of differential pumping, in analogy to Froud et al. [67], but with an additional modification to the capillary to further reduce the unwanted section of decaying pressure behind the effective interaction region. The design of the capillary will be described in detail also in section 4.1. Also, we will discuss the diagnostics we developed to measure the harmonic beam profile and the spectrum.

With this setup we successfully generated high harmonics and recorded spectra. In section 4.2 examples of the measurements will be described. Furthermore, with this setup we performed a first experiment that demonstrates the working of HEx in a guided-wave geometry for which we employed a mixture of He and Xe gas. The basic tests of HHG and HHG with HEx will be presented in section 4.2 .

\subsection{Experimental setup}

For the experiments on HHG we made use of part of the output of the 12 TW laser at LPNO (see chapter 1.1). At a later stage, the full pulse energy $(\approx 0.5 \mathrm{~J})$ may be employed as well, e.g., to generate XUV pulses with record intensities after focusing. It should be noted that the maximum pulse repetition rate of this laser is $10 \mathrm{~Hz}$, this is much less than that usually employed for HHG with repetition rates in the range of $\mathrm{kHz}$, however, where the pulse energy is limited to a few $\mathrm{mJ}$. 
For our HHG experiments we have introduced a separate beam line into the laser system. There we couple out the stretched laser pulses after the first multi-pass amplifier and behind a spatial filter made of a tapered glass capillary. The filter is used to improve the spatial beam profile after the amplification process $^{1}$. The output is sent to a compressor designed for a maximum energy of about $50 \mathrm{~mJ}$. The measured fluctuations in the pulse energy are $10 \%$ and are mainly caused by operating the multi-pass amplifier below saturation [47]. An advantage of this mode of operation is that the laser output pulses posses a transverse beam profile which is close to a Gaussian behind the spatial filter. Saturated amplification would generate a beam profile closer to a flat top. These differences in beam profiles can be of importance for HHG. A Gaussian profile is close to that required for an optimal coupling into the fundamental mode of a capillary waveguide, as described in section 2.2.3. A flat-top beam profile, instead, is expected to excite also higher-order transverse mode. The resulting mode beating would cause a longitudinally modulated intensity pattern in the waveguide which is of interest for QPM (see section 2.2.3 and [102]).

Behind the compressor (efficiency of about $60 \%$ ), a beam-splitter reflects $90 \%$ of the pulse to a separate optical table that contains the experimental apparatus for HHG. The remaining $10 \%$ is sent to a, so-called, GRENOUILLE (an acronym for GRating-Eliminated No-nonsense Observation of Ultrafast Incident Laser Light E-fields) ${ }^{2}$, which measures the duration of the compressed pulses and which is used to align the compressor. To vary the energy of the pulses, we inserted neutral density (ND) filters (in front of the compressor) or adjusted the time delay between the stretched pulse and the pulses of two Nd:YAG lasers that pump the multi-pass amplifier crystal. The maximum available energy of the compressed pulses is approximately $25 \mathrm{~mJ}$. Pulses with an energy of only about 1 to $3 \mathrm{~mJ}$ were focused into the gas-filled capillary for the generation of high harmonics when using pressure-tuned phase matching, for which it is required to avoid too strong ionization (see section 2.2.2). At a later stage, higher energies will be employed when using capillaries with a larger inner diameter or when HHG from ions will be investigated.

Initially, the pulse duration was estimated to be about 90 fs full-width-athalf-maximum (FWHM) and the focal spot had an elliptical transverse profile (close to a Gaussian) with a waist of $52 \times 60 \mu \mathrm{m}$ when using a plano-convex lens with a focal distance of $100 \mathrm{~cm}$. The pointing stability of the focal spot position was measured to be within $5 \mu \mathrm{m}$. These were the parameters used during most of the test experiments. At a later stage (after removal of the wavefront tilt [172] and improved calibration of the GRENOUILLE), the pulse duration was 49 fs FWHM and the focal spot profile was round, with a waist of about $50 \mu \mathrm{m}$.

\footnotetext{
${ }^{1}$ For LWFA experiments, the laser pulses are further amplified and finally compressed in a vacuum compressor designed for a pulse energy of 1 Joule.

${ }^{2}$ This is basically a Frequency-Resolved Optical Gating device (FROG) which can characterize each individual pulse with regard to its temporal pulse shape and any time-dependent phase variation.
} 
In the following paragraphs a detailed description of the apparatus constructed for HHG will be given. An overview is shown in figure 4.1, where the main components have been indicated.

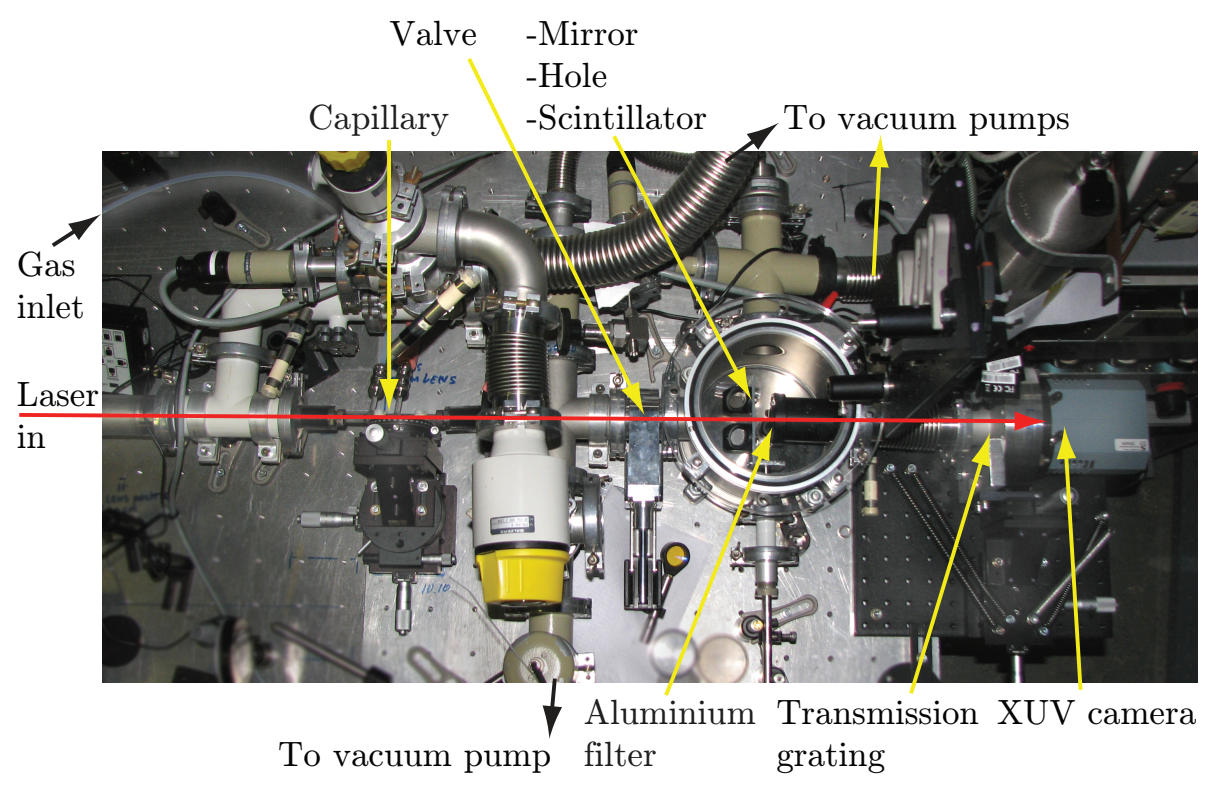

Figure 4.1: Top view of the HHG setup at LPNO. The IR drive laser enters the system from the left side, passing through the gas-filled capillary where it generates high harmonics which copropagate with the drive pulse. The IR beam is blocked by the aluminium filter. Part of the harmonic radiation is transmitted (passed through a transmission grating or opening) and is subsequently detected by the XUV camera to measure the spectrum or the spatial profile. Also, in order to characterize the IR beam or the harmonic beam, respectively, a mirror or a scintillator plate can be placed in the beam path via a feed-through axle. The whole setup is maintained at vacuum with a background pressure of about $10^{-5}$ mbar.

To avoid absorption of the generated XUV radiation, most of the beam line is maintained at vacuum via vacuum tubes and connections arranged around the laser beam propagation axis. This configuration has several advantages. First, it is flexible and allows modifications to be made relatively easily and fast during testing of the setup. Second, it allows the aligning of the capillary onto the laser beam, thus simplifying the alignment procedure and the measurements. Also for the purpose of easy alignment, we decided to use a transmission grating $^{3}$ (location indicated in the photo) to measure the harmonic spectrum.

\footnotetext{
${ }^{3}$ This grating was put at our disposal by prof. dr. F. Bijkerk from FOM Rijnhuizen.
} 
A reflecting grating would alter the path of the harmonic beam and would complicate the alignment of the system significantly, especially when focusing optics (such as a toroidal mirror) would be used. Furthermore, a transmission grating allows a twofold mode of operation, allowing the measurement of the harmonic spectrum and the harmonic beam profile with the same XUV camera.

The third advantage of our configuration is that, in order to reduce reabsorption of the harmonic radiation by the gas flowing out of the capillary, differential pumping could be implemented in the capillary section in a simple manner via slits cut in the capillary wall. This part of the setup, the differential pumping and the coupling of the laser into the capillary, will be described in more detail in subsection 4.1.1. An additional feature, shown in the figure, is the valve in the center of the setup to disconnect the capillary system from the vacuum chamber, so that one part can be maintained at vacuum, while modifications (in air) were made on the other part.

It can be seen in figure 4.1 that the diagnostic equipment consisting of an XUV CCD camera (ANDOR D0420-BN) and a small cylindrical vacuum chamber is located on the right side of the central valve. The vacuum chamber holds the axis of a feed-through axle with an aluminium plate that carries a mirror and a scintillator plate. The mirror is used to reflect the IR laser upwards to a standard CCD camera (not shown in the photo) to inspect the laser transmission through the capillary, and is used for fine tuning the alignment of the capillary. The scintillator plate is used to detect whether XUV radiation is generated and for a fast characterization of the harmonic beam profile. The plate is covered with a sodium salicylate layer that emits visible blue light when it is illuminated with XUV radiation. Between the mirror and the scintillator plate, a hole allows the beam to pass to a spectral filter that blocks the IR drive laser and transmits part of the harmonic radiation to the XUV camera. For more details on these diagnostic tools, the reader is referred to subsection 4.1.2.

To provide the nonlinear medium for HHG, gas flows into the capillary via a small tube connected to a large supply bottle. Different gas bottles and a vacuum pump are connected to this supply bottle to enable gas selection and pressure adjustment. In our experiments we used Xe, Ar, He and also a mixture of $\mathrm{He}$ and Xe. A Barocell meter measures the pressure inside the supply bottle. This pressure is used as a reference for the pressure inside the capillary. However, the exact pressure inside the capillary is not known and, according to our estimates and calculations by Froud [67], the absolute value may be lower by a fixed factor of about 0.7 than that indicated by the Barocell.

\subsubsection{Capillary and laser coupling}

The capillary we used had an inner radius, $a$, of $75 \mu \mathrm{m}$. The capillary radius and the focal length of the lens were chosen such that the smallest waist of the, initially, somewhat elliptical focal spot was close to the optimum coupling parameter of the fundamental mode $\left(w_{0}=0.64 a\right)$. Any light coupled into highorder transverse modes then rapidly diminishes in the capillary. The excitation 
of transverse modes and corresponding mode beating could easily be detected as a spatially modulated, visible fluorescence along the capillary. Later, the mode beating was further minimized by removing a residual wavefront tilt via improved alignment of the stretcher and the compressor because this yields an almost circular profile of the focal spot.

(a)
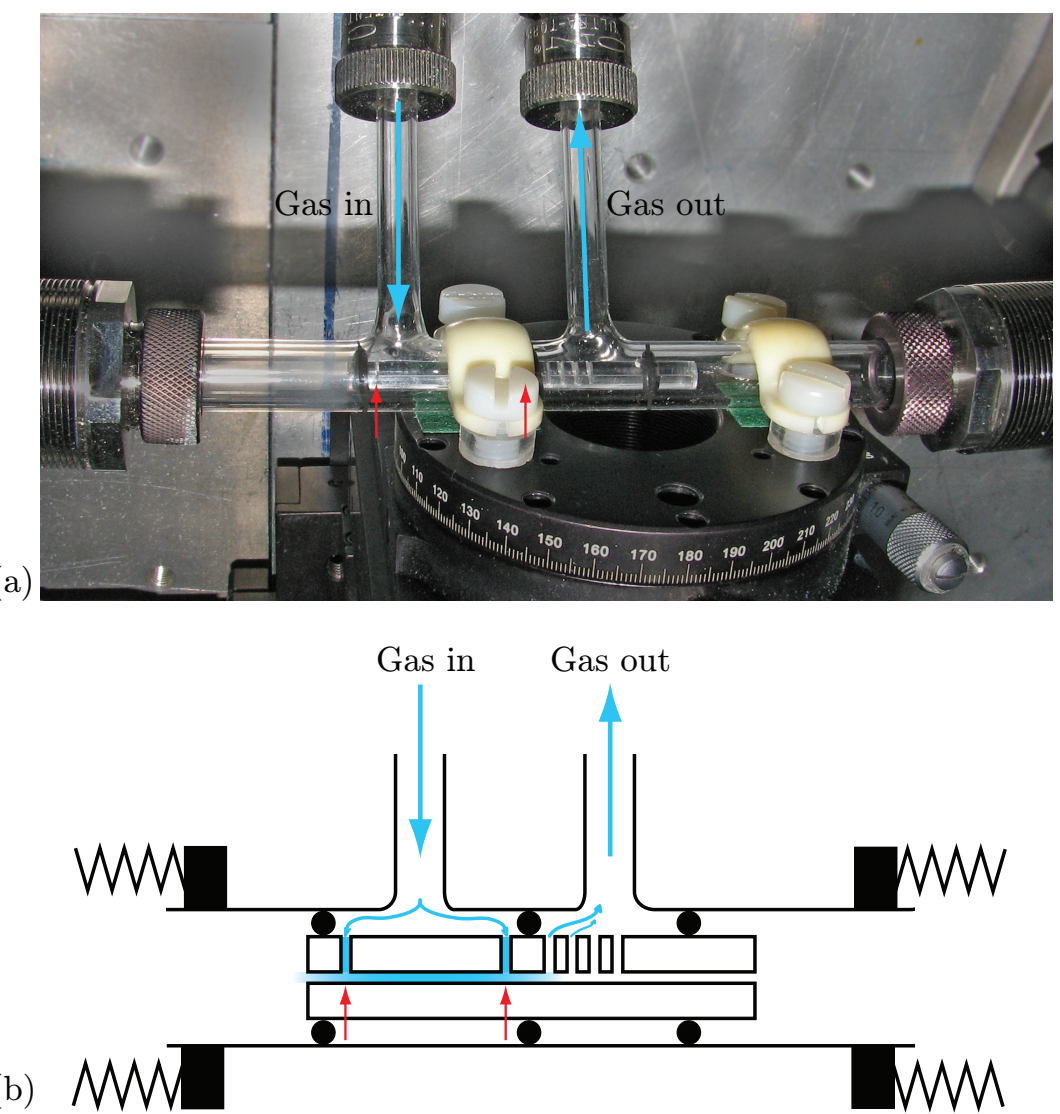

Figure 4.2: Photo (a) and schematic picture (b) of the gas-filled capillary. A homogeneous gas pressure is established in between the two gas inlet slits, indicated by the red arrows. Differential pumping via four outlet slits enables to reduce the pressure behind the capillary to a low value in the order of $10^{-5}$ mbar.

Figure 4.2 shows a photo and a schematic drawing of the capillary and its mounting. The capillary is made of borosilicate, is $52 \mathrm{~mm}$ long and has an outer diameter of $4.3 \mathrm{~mm}$. The capillary is fitted inside a custom-made glass tube to allow gas to flow into and out of the capillary. The glass tube is connected to the vacuum system via two flexible bellows, to enable the alignment 
of the capillary onto the propagation axis of the laser beam. Initially, for this alignment, the capillary system was connected onto a set of translation and rotation stages for horizontal, vertical, planar, azimuthal and longitudinal motion (along the beam axis). This configuration was used during most of the test measurements. However, to obtain the highest transmission of the drive laser, it turned out that the alignment of the capillary was very critical with respect to the angle of incidence. This was concluded from measurements of the transmission of the drive laser through capillaries with several different lengths between 1 and $11 \mathrm{~cm}$. The transmission was found to be between 80 to $95 \%$, almost independent of the length of the capillaries. It should be noted that it was difficult to align the shorter capillaries with high accuracy due to their larger acceptance angle for coupling and propagation through the capillary, this may not necessarily result in the highest transmission. The accuracy of the alignment was thereafter improved by positioning two $\mathrm{x}-\mathrm{y}-\mathrm{z}$ translation stages at the planes of entrance and exit of the capillary.

As is illustrated in figure $4.2 \mathrm{~b}$, gas flows into the capillary via two slits ( $0.4 \mathrm{~mm}$ wide and indicated with red arrows in the figure). The slits were cut in the capillary wall with a low-speed diamond saw. The two inlet slits are separated by a distance of $20 \mathrm{~mm}$ and a homogeneous pressure distribution is expected to be achieved in the region between the two slits [67]. According to calculations by Froud [67], who also used a capillary for HHG, there is a linear pressure drop on the left and right side of the homogeneous pressure region and a vacuum at the interfaces. This section of unwanted decaying pressure could result in detrimental re-absorption of the harmonic radiation. In order to reduce the length of this section and yet minimize the gas leakage into the vacuum system behind the capillary, we implemented differential pumping in a novel manner, using 4 outlet slits with a $2 \mathrm{~mm}$ spacing cut in the capillary itself. Three compressed viton O-rings hold the capillary in place in the glass tube and provide gas sealing between the sections with different pressures. In this way, the backing pressure in the vacuum section behind the capillary and inside the small vacuum chamber was brought to low values in the order of $10^{-5}$ mbar. This pressure remained the same when the gas supply into the capillary was opened. This observation indicates that the differential pumping via the four slits works very effectively compared to the widely used standard arrangement that contains only two internal capillary inlets and no outlets. Another indication that the differential pumping is functioning properly can be seen from the visible light inside the capillary. If the gas inside the capillary is illuminated with laser pulses of more than $3 \mathrm{~mJ}$ energy, the gas is strongly ionized and the plasma emits bright light (visible with the eye) due to recombination of present laser-induced ionized gas. This emission only occurs up to the first slit of the 4 outlet slits, this indicates that there is hardly any gas beyond this point.

In order to generate high harmonics in the gas-filled capillary, the drive laser pulses have to be coupled into the capillary. This is done using a lens with a focal distance of $100 \mathrm{~cm}$ (positioned in front of the visible part of the setup in 
the photo in figure 4.1). This focal length was selected to match the size of the focal spot with the capillary radius mentioned above. The lens is placed on an $x-y-z$ translation stage and can be tilted in the azimuthal direction for fine alignment. In order to have a well-defined axis for the laser propagation onto which the whole setup has been aligned, and to obtain reproducible results each measurement, sets of diaphragms are used to accurately mark the laser beam propagation. The focused laser pulses then enter the vacuum system via an anti-reflection coated entrance window before being coupled into the gas-filled capillary. After the capillary is aligned with regard to the laser beam, gas can be flowed into the capillary to generate high harmonics which are then detected and the beam profile and the spectrum are characterized.

\subsubsection{Diagnostics}

In order to give insight in the results of the test measurement on HHG, a more detailed description of the diagnostic part of the setup will be given next.

The first indication that harmonic radiation in the XUV regime is generated is acquired by looking at the scintillator plate, which emits blue light when irradiated with XUV light. Then, either the characterization of the harmonic beam profile or the spectrum is achieved by a combination of a spectral filter, removable transmission grating and an XUV camera. An aluminium filter is used to block the IR beam and transmits part of the harmonic spectrum between wavelengths of 20 and $80 \mathrm{~nm} .{ }^{4}$ During the first tests, the filter was a $150 \mathrm{~nm}$ thick aluminium foil on a support mesh. This mesh, which absorbs XUV radiation, was distinctly visible in the detected harmonic beam profile. The very short scales of the observed diffraction pattern due to the wires of the support mesh, confirmed via numerical calculations, indicated that the radiation was indeed in the range of the XUV. In order to record the unperturbed harmonic beam profile and determine the divergence of the beam, we used a slightly thicker $(200 \mathrm{~nm})$ but freestanding aluminium filter. This filter transmits the XUV radiation with a transmission efficiency of about $60 \%$. To prevent scattered light, mainly IR light from the drive laser beam, from falling onto the XUV camera, the filter is integrated into a light baffle, made of two coaxial, internally-threaded tubes. The baffle is constructed such that gases can be effectively pumped through to achieve vacuum on the side of the XUV CCD camera.

The harmonic radiation is detected with a 16 -bit XUV CCD camera (ANDOR D0420-BN). The camera has a quantum efficiency in the order of 20 to $30 \%$ for wavelengths between 20 and $80 \mathrm{~nm}$ that pass the aluminium filter. The effective detection area of the camera is $26.7 \times 6.7 \mathrm{~mm}$ with $1024 \times 256$ pixels of $26 \times 26 \mu \mathrm{m}$. Data is recorded by intergrating over a larger number of shots, typically 100 to 1000 shots (i.e. within 10 to 100 seconds at $10 \mathrm{~Hz}$ ). In order to reduce the thermal noise level, the camera is cooled to typically $-20{ }^{\circ} \mathrm{C}$.

To measure the harmonic spectrum, we used a transmission grating on a

\footnotetext{
${ }^{4}$ http://henke.lbl.gov/optical_constants/
} 
custom-made mount in the vacuum flange in front of the camera. In order to detect the full spectral range between 20 and $80 \mathrm{~nm}$, the transmission grating is positioned $20.2 \mathrm{~mm}$ in front of the camera. The mount also has a $5 \mathrm{~mm}$ opening hole next to the grating to record the beam profile with the camera. In order to switch between a measurement of the spectrum or of the beam profile, the XUV camera and mount are positioned onto a translation stage. The harmonic output is sufficiently high enough to record single-shot spectra (without the weakest cut-off harmonic orders), which is helpful for aligning the grating on the propagation axis of the XUV beam.

The transmission grating, fabricated at DIMES [173], is made of $300 \mathrm{~nm}$ thick silicon nitride with a high grating constant of 10.000 lines per mm (a slit periodicity of $100 \mathrm{~nm}$ ) for a strong diffraction in spite of the short wavelength of the XUV radiation. Rigidity and strength to this delicate fine structure is provided by a coarse support structure with a periodicity of $1 \mu \mathrm{m}$ orthogonal to the fine structure. This support structure also acts as a grating with smaller diffraction angles. The efficiency of the grating in the first order diffraction angle that is recorded with the camera is $6.5 \%$. The grating area is $5.1 \times 1.2 \mathrm{~mm}$. The harmonic spot size falling onto the grating is typically slightly less than $1 \mathrm{~mm}$. This means that the harmonic orders, as recorded with the camera, possess approximately the same size as the incoming beam. The spectra presented in section 4.2 were recorded using this configuration. To avoid the large size of the harmonic orders limiting the spectral resolution of the spectrometer at higher orders (which are closely spaced from each other and eventually overlap), an additional slit of $200 \mu \mathrm{m}$ wide was placed in front of the grating at a later point. Using the slit, which effectively increases the spectral resolution, longer integration times were used to compensate for the reduced throughput.

\subsection{Results}

In the following paragraphs, examples of the experimental results will be presented. We irradiated Xe, Ar, He and gas mixtures of He and Xe, with lowenergy laser pulses of 0.7 to $1.5 \mathrm{~mJ}$, which corresponds to estimated intensities of about $10^{14} \mathrm{~W} / \mathrm{cm}^{2}$. Figure 4.3 shows examples of recorded spectra using Xe, Ar and a He-Xe mixture, where the highest harmonic intensity is normalized to unity. The recorded spectra are integrated over 100 shots. Other experimental parameters are given in the figure caption. The scale for the wavelength is obtained from the well-known grating equation and the geometry of the setup. In these examples, the width of the harmonic peaks are correlated to the width of the incoming spot onto the large area of the transmission grating, as mentioned above.

As can be seen from figure 4.3, we generated high harmonics in Xe and $\mathrm{Ar}$ with a minimum wavelength of approximately $36 \mathrm{~nm}$ and $24 \mathrm{~nm}$ respectively. These correspond to maximum harmonic orders of 21 and 33. With Xe and Ar we observed that there is an optimum gas pressure for maximizing the harmonic output, as expected based on the phase-matching condition described 


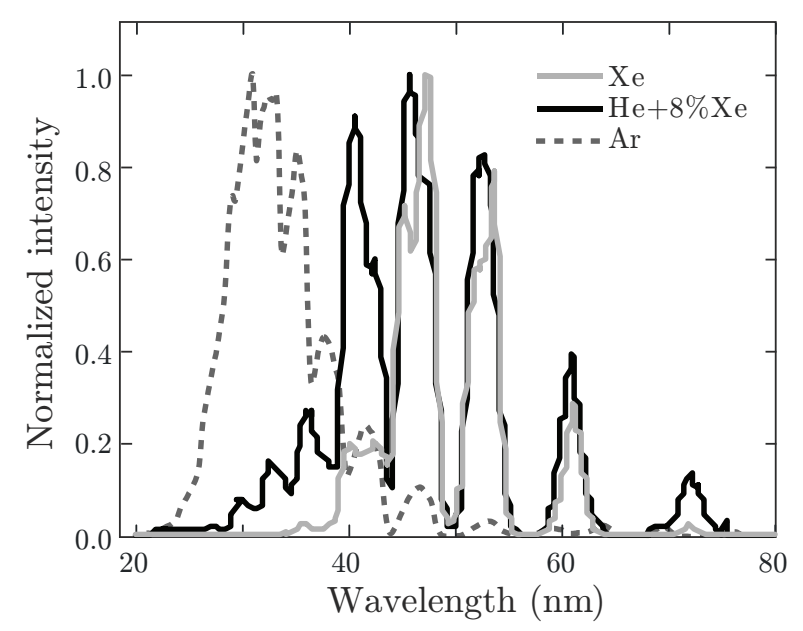

Figure 4.3: Harmonic spectra for pure Xe (27 mbar), pure Ar (64 mbar) and a mixture of He plus $8 \%$ Xe (110 mbar). With pure He no harmonic signal could be observed at all. Xe and the mixture were irradiated with drive laser pulses of $0.7 \mathrm{~mJ}$ and Ar with $1.4 \mathrm{~mJ}$. The intensity of the curve of the He-Xe mixture is enlarged by a factor of 8 with respect to the curve of Xe, to show the higher orders. The spectra show no apparent plateau region due to reabsorption of the generating medium and phase-matching of only a few harmonic orders. The maximum generated harmonic order with $\mathrm{Ar}$ is 33 and with $\mathrm{Xe}$ is 21 (enlarging the scale shows no higher orders). The maximum order with the He-Xe mixture is 27. The generation of the harmonic orders above the $21^{\text {st }}$ order in the gas mixture would be due to the harmonic excitation of He atoms by XUV photons generated from Xe. The horizontal axes contains estimated values for the wavelength.

by equation 2.17. The optimum pressure found with Xe was approximately 25 mbar and with Ar approximately 65 mbar, which is in reasonable agreement with the theoretical prediction (within the experimental error). Up to the optimum pressure, we observed that the spot size of the harmonic beam becomes smaller with increasing pressures, which is consistent with previous observations of an improved spatial coherence of the harmonic beam when the HHG process is phase-matched $[49,83,85]$. From the measured spot profile and the distance between the capillary and the XUV camera, we deduced the divergence of the beam to be approximately $1 \mathrm{mrad}$ with a pointing stability of $0.15 \mathrm{mrad}$. Higher drive pulse energies resulted in less stable harmonic beams, most likely due to instabilities induced by ionization [50,174].

The maximum harmonic orders we recorded are in agreement with the ex- 
pected value for the cut-off frequency (equation 2.13). Using this equation, the intensity that would correspond with the maximum harmonic order of 33 for $\mathrm{Ar}$ is about $1.9 \cdot 10^{14} \mathrm{~W} / \mathrm{cm}^{2}$. The maximum order of 21 for Xe would suggest a peak intensity of about $1.1 \cdot 10^{14} \mathrm{~W} / \mathrm{cm}^{2}$. These values are in the range of what we estimated. The maximum observed orders are also consistent with previous experiments performed by, for example, Durfee et al. who used comparable intensities with a gas-filled hollow-fiber with a radius of $75 \mu \mathrm{m}$ as well [83]. Compared to the experiments of Durfee, our measured values for the pressures are somewhat higher and the phase-matching pressure peak is somewhat broader. Also, our measured optimum pressure is about a factor of 2 higher than that obtained by Naumov et al. in calculations on a gas-filled hollow-fiber with a radius of $75 \mu \mathrm{m}$ (where no ionization was assumed) [84]. The reason for our higher value can be twofold. First, the pressure we measure with the Barocell may be higher than the pressure inside the capillary, as was discussed above. Second, we use laser pulses of a longer duration. As discussed in chapter 2.2.2, for the same peak intensity, longer laser pulses result in a higher degree of ionization. This can make the harmonic generation efficiency less sensitive to the pressure (broadens the peak) and causes the optimum pressure to shift towards higher values [84].

What is also consistent with previously reported data $[83,175]$, is the shape of the spectra of Xe and Ar that show no apparent plateau region. This can be ascribed to the re-absorption of these harmonics in the generating medium and, also, that the spectral acceptance bandwidth of phase-matching comprises only a few harmonic orders. This results in spectra that, in our experiments, show a maximum output at the $17^{\text {th }}$ harmonic order for Xe and the $27^{\text {th }}$ order for Ar. We obtained an estimated value for the efficiency by taking into account the grating efficiency, the quantum efficiency of the CCD, the number of electrons per incident photon, and the transmission of the filter. Currently, the highest efficiency we obtained for the above-mentioned harmonic orders is estimated to be around $10^{-7}$. This value is comparable to what was obtained by others $[19,76]$. However we expect a further increase in the near future using shorter drive pulses [87].

The experiments described so far were performed to test the basic performance of the apparatus. These experiments verified that the experimental parameters for HHG were suitably chosen and that the output is quantitatively consistent with the state of the art. The experiments described next, however, form a first step into unexplored ranges. Specifically, the experiments aimed at the first demonstration of harmonic excitation (HEx) in a guided-wave geometry. Such demonstration in a guided-wave geometry is of interest to explore whether a further increased output is possible, in addition to the formerly proven improvement of such a geometry over configurations using freely propagating beams (see section 2.2.3). Additionally, our future experiments with HEx in ions will be based on a waveguiding geometry as well, which requires a prior test of the working of HEx in a waveguide as a preparation. 
The demonstration was done in two steps. As the first step we provided a situation where a gas with a high ionization potential (here helium) did not generate high harmonics in the inspected range $(20$ to $80 \mathrm{~nm}$ ), although waveguiding increased the interaction length. We created this situation by irradiating helium in the capillary with sufficiently low pulse energies, between 0.7 and $1.5 \mathrm{~mJ}$. No harmonics were observed from helium, not even when the integration time was increased. Then, as the second step, we added $8 \%$ Xe to the He gas. This percentage of Xe was chosen because this was the optimum ratio found by Takahashi et al. [59] (see section 2.2.3). However, we note that for our experiment the optimum ratio can be different and has yet to be determined. With this setting we observed HEx in a guided geometry, which can be seen as follows.

The harmonic spectrum we acquired by using the He-Xe gas mixture is shown in figure 4.3. It should be noted that in this experiment the pressure (110 mbar) was too low to reach the optimum pressure. This resulted in a broader harmonic spot profile and a lower harmonic yield as compared to Xe, however, we expect a higher yield when optimizing the pressure and gas mixing ratio. The maximum harmonic order of 21 we observe with pure Xe is in accordance with the result of Takahashi. If we assume that the intensity was $1.1 \cdot 10^{14} \mathrm{~W} / \mathrm{cm}^{2}$, as was estimated above, from He one could expect, theoretically, to generate high harmonics of up to the $29^{\text {th }}$ order, which is close to the maximum order of 27 that we observe with the He-Xe mixture (see figure 4.3). At this drive intensity, the harmonic orders above the $21^{\text {st }}$ order cannot be attributed to Xe atoms. Instead, they have to be emitted from the He atoms. This result clearly demonstrates the working of HEx under waveguiding.

\subsection{Discussion}

Using a capillary waveguide for HHG has two main advantages. First, such a guided geometry results in a higher harmonic output and a higher spatial coherence compared to a gas jet. A second advantage is that this geometry subsequently enables a relatively straightforward extension towards HHG in ions in a plasma waveguide (capillary discharge). This is the next step to increase the maximum harmonic order. Also, investigations will be made to increase the efficiency of the cut-off harmonics using harmonic excitation (HEx) in ions. In particular, we will use a specially designed capillary waveguide that consists of two sections, both of which can be separately filled with gas and hold a discharge to provide ions. With such a capillary, several combinations are feasible to perform HHG via HEx. Four examples are illustrated in figure 4.4.

As a first preparation to HHG experiments via HEx in ions, we will investigate whether HEx also works using separate gas-filled sections in a capillary (e.g. Xe in the first section and He in the second), as illustrated in figure 4.4a. Then, high harmonics generated in the first section would copropagate with the drive laser and excite atoms in the second section so that harmonics of a higher frequency can be generated. This approach is to be compared with 
(a)

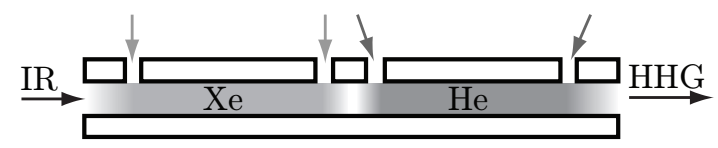

(b)

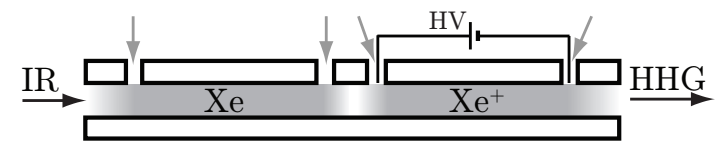

(c)

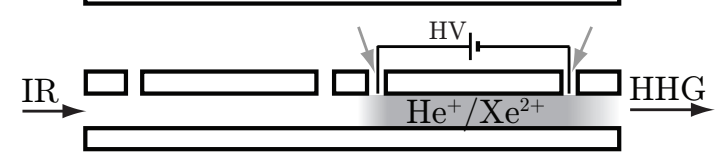

(d)

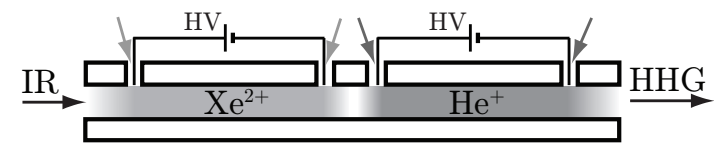

Figure 4.4: Schematic drawings of possible implementations of HEx in a guided-wave geometry. A high-voltage (HV) discharge is used to produce ions. In the drawings only the inlets are shown while outlet slits for differential pumping have been omitted for better readability. An explanation of the four cases (a to d) is given in the main text.

HEx in a gas mixture (single section filled with He and Xe). The next step (figure $4.4 \mathrm{~b}$ ) is to fill the capillary with a gas, for example $\mathrm{Xe}$, where the first section contains neutral gas and the second section contains ions obtained via the discharge.

Another approach to perform HEx in ions will be to fill the discharge section with a gas mixture (as in figure 4.4c), for example He plus Xe, and produce $\mathrm{He}^{+}$and $\mathrm{Xe}^{2+}$ ions. Then, harmonics generated from $\mathrm{Xe}^{2+}$ have to excite the $\mathrm{He}^{+}$ions. This situation can be compared to a case where the ions of the two different types of gases are contained in separate sections, as in figure $4.4 \mathrm{~d}$.

Additionally to the enhancement via HEx, to ensure efficient harmonic generation from the ions, a technique of quasi-phase-matching has to be employed. For this, the most promising method seems to be multi-mode QPM. This method has to be optimized for our configuration.

Finally, when these methods result in a stable, highly efficient, output of high harmonics, nonlinear optics in the XUV regime could be performed and the functionality of XUV optics, which are being developed in our group, could be characterized.

Several steps had to be made in preparation to the above-mentioned plans. This chapter described the most basic step, which was to realize and test an experimental apparatus for HHG using the TW laser at LPNO.

We successfully constructed and tested an experimental setup to generate 
and characterize high harmonics from gas-filled capillaries. A configuration of the capillary section was made where we implemented a novel approach of differential pumping using outlet slits in the capillary in order to minimize re-absorption of the high harmonics behind the interaction region. For the XUV diagnostics we implemented a shiftable section comprising a transmission grating and an XUV camera. This enabled the measurement of the harmonic spectrum and also the harmonic beam profile. We recorded spectra from HHG in Xe and Ar. These spectra are consistent with previously reported results, which illustrates the proper functioning of our apparatus. Furthermore, we can record single-shot spectra, which illustrates the relative high efficiency of HHG in our setup. We expect that the efficiency can be further improved using optimized laser pulses. As a step towards a novel direction, we demonstrated harmonic excitation for the first time in a guided geometry using a gas mixture of $\mathrm{He}$ and $\mathrm{Xe}$ in a capillary waveguide. This is different from the work by Takahashi et al. who used a gas cell and loose-focusing [59].

Recently, further improvements were made to our system. In Xe we recorded a first spectrum with an enhanced resolution using a slit of $200 \mu \mathrm{m}$ wide in front of the grating. The spatial quality of the drive laser radiation has been further improved. Wavefront tilt and the associated ellipticity of the focal spot profile was eliminated after a modification to the stretcher, the amplifier systems and, the compressor. Mode beating inside the capillary is now absent, which illustrates a proper coupling of the laser pulse into the capillary. The pulse duration is now shortened, about 50 fs FWHM, which is due to the removal of wavefront tilt as well. With the shorter pulses we observe now that that less pulse energy is required (approximately a factor of 2) to generate high harmonics. To avoid damage to the aluminium filter with the shorter pulses, we inserted an additional vacuum section between the valve and the small vacuum chamber. When using higher pulse energies to generate harmonics from, e.g., ions, two anti-reflection coated dielectric plates will be inserted in the beam path under grazing incidence that will transmit the IR laser pulse and reflect the harmonic radiation.

With the current improved configuration, experiments are being executed to measure systematically the harmonic beam profile and the harmonic spectrum from $\mathrm{Xe}, \mathrm{Kr}, \mathrm{Ar}, \mathrm{Ne}$ and $\mathrm{He}$, which includes a more detailed and quantitative characterization of HEx in a guided geometry, before investigating guided HHG in ions via HEx. 
Richard Feynman (1918 - 1988)

\section{Generating high-order harmonics and fast ions from solids}

In this chapter, our experimental results with the TW laser at PHI on ion acceleration and high-order harmonic generation from solid targets are described. Compared to the generation of high harmonics in gases, as was described in the previous chapter, high-order harmonic generation from solids (HOHG) bear a higher potential, such as the absence of a cut-off. In addition, the laser interaction with solid targets has great potential for the acceleration of ultrashort highly energetic ion beams. However, these processes require more involved laser systems, in particular the ultra-high pulse contrast described in chapter 3. Actually, the requirements lie at the front of current laser technology, but a laser optimized to such specifications is operating at PHI in CEA Saclay, France, and access was made available to us in collaboration.

The idea of exploiting the so-called transparent regime for ion acceleration, via extremely thin foils with thicknesses in the order of the skin depth of the laser light, appears promising because the theory predicts that the transparent regime would provide an enhanced flux and energy of the accelerated ions. However, this prediction is based on numerical calculations that may not have included all of the relevant physical effects present and still requires an experimental demonstration. In order to test the validity of this model, we experimentally investigated ion acceleration in this transparent regime for the first time using extremely thin targets (nanofoils) and ultra-high contrast, high-intensity laser pulses.

The predicted enhancement of ion acceleration in this transparent regime is based on the expectation that a higher temperature and density of hot electrons will be obtained during the interaction of the thin target with the laser pulse. 
Hot electrons can also be responsible for the generation of high-order harmonics since the initial absorption mechanism of the laser pulse energy is the same for ion acceleration and HOHG because, with ultra-high contrast pulses, these processes appear simultaneously. Considering the possible enhancement of ion acceleration, the question arises whether, and to what extent, HOHG can also be improved by utilizing extremely thin foils. A possible answer is that HOHG might be improved because the excursion amplitude and the velocity of the plasma electrons may be increased due to a smaller plasma restoring force associated with thinner foils.

Based on these considerations and in order to gain more insight into the process of both HOHG and ion acceleration, we performed simultaneous experiments on the two processes using extremely thin foils. This also helped us to identify the actual requirements on the laser and the target for optimum output. However, extremely thin foils are very fragile unlike thick $(\gtrsim 10 \mu \mathrm{m})$ foils which can easily be supported over large areas in a freestanding manner and can sustain many shots with a high-intensity laser without causing damage to the remainder of the foil. It seems that this expected problem had prevented the investigation of thin foils as desired, down to a few nanometer, by irradiation with high-intensity lasers. To overcome this, a special way to mount such nanofoils is required. We investigated a method to prepare freestanding nanofoils. We fabricated special support frames and optimized the preparation procedure as it turned out that it is not trivial to prepare nanofoils that are physically flat, a requirement which must be met in order to have a well-defined angle of incidence for the laser pulse and of the proton emission and harmonic radiation in the experiments. We begin this chapter with a detailed description of the preparation of such nanofoils in section 5.1, which is also useful as a guide for repetition of the preparation. A description of the experimental setup at PHI and the diagnostics used to measure the data will be given in the second section.

Section 5.3 presents the experimental results. We start in section 5.3.1 with a description of the experimental study of the coherence of HOHG. Although HOHG from solid targets (overdense plasmas) results in collimated $\mathrm{X}$-rays beams, this property alone does not guarantee spatial coherence of the radiation [53]. We performed an experiment with bulk solid targets that verifies that HOHG by coherent wake emission (CWE, see section 3.3.2) leads to coherent radiation [176]. In addition, this experiment allowed the study of the dynamics of the plasma electrons during the interaction with the laser. This is of great importance in understanding more of the physical details of the interaction process with the laser, for example the absorption, in order to explain the properties of the subsequent phenomena, including ion acceleration and HOHG.

The experimental results on ion acceleration and HOHG obtained with the nanofoils will be described in sections 5.3.2 and 5.3.3. We performed two such experiments, one experiment with the $10 \mathrm{TW}$ laser (UHI 10) at PHI and a second experiment after the laser was upgraded to 100 TW (UHI 100). We will 
show that the experimentally observed dependence of the maximum proton energy on the foil thickness differs from the theoretically predicted trend of the models that have been employed so far. We also generated high-order harmonics from extremely thin foils, however, no enhancement of the efficiency was found under present experimental conditions. The results suggest that, for improving HOHG, it is important to take into account the damage threshold of the target material and the contrast of the pulse because these determine the initial scale length of the plasma gradient with which the main pulse interacts.

Additional insight into the generation of fast ions and high-order harmonics can be acquired from a comparison with numerical calculations. In order to interpret our experimental data, we performed numerical modeling with a particle-in-cell (PIC) code. These results are discussed in section 5.4. We will show that our experimental data on ion acceleration agrees with our improved model which takes into account the limited expansion of the foils due to the high pulse contrast. In addition, we will show that the prediction of an enhancement in the transparent regime for ion acceleration also holds for HOHG.

\subsection{Foil preparation}

In order to study the interaction of high-intensity lasers with foils which have thicknesses in the order of the penetration depth of light, for example, approximately $140 \mathrm{~nm}$ for neutral carbon and only $7 \mathrm{~nm}$ when carbon is fully ionized, we developed targets that contain freestanding nanofoil and allow multiple shots of high-intensity laser pulses.

We used carbon nanofoils, because foils thinner than $10 \mathrm{~nm}$ were only commercially available when made of carbon. Samples of freestanding carbon nanofoils are prepared with thicknesses varying between 1 and $120 \mathrm{~nm}$ and mounted on a support frame (sample holder). For this, arc-evaporated carbon films from ACF Metals are floated off from glass microscope slides in deionized water at room temperature [177], and then picked up onto a frame, in which arrays of micro-holes have been fabricated. We performed a series of experiments with two different types of frames. The design and fabrication of these home-made frames is described first, followed by a more detailed description of the preparation procedure used to obtain flat freestanding nanofoils.

The frame was designed such that an extremely thin carbon foil is supported and that many laser shots can be fired onto the target. The frame design was determined by the strength of the carbon nanofoils. The carbon foils we used have two parameters to the manufacturers specifications, the areal density in $\mu \mathrm{g} / \mathrm{cm}^{2}$ and the mass density in $\mathrm{g} / \mathrm{cm}^{3}$. The mass density of arc-evaporated carbon foils with an areal density larger than $0.5 \mu \mathrm{g} / \mathrm{cm}^{2}$ is $2.01 \pm 0.02 \mathrm{~g} / \mathrm{cm}^{3}$, and the density is $1.8 \mathrm{~g} / \mathrm{cm}^{3}$ for foils of $0.5 \mu \mathrm{g} / \mathrm{cm}^{2}$ and lower $[178,179]$. The mass density can be used to convert the areal density to the thickness (multiply with a factor 5 to convert the areal density in $\mu \mathrm{g} / \mathrm{cm}^{2}$ to the thickness in $\mathrm{nm})$. For the experiments discussed in this chapter, foils have been prepared that have an areal density between 0.2 and $24.0 \mu \mathrm{g} / \mathrm{cm}^{2}$, corresponding to 
thicknesses between approximately 1 and $120 \mathrm{~nm}$. A rough limit for the largest aperture that can be covered with a freestanding carbon foil is $1 \mathrm{~mm}$ diameter for each $1 \mu \mathrm{g} / \mathrm{cm}^{2}$ [178]. This means that a foil of $1 \mathrm{~nm}\left(0.2 \mu \mathrm{g} / \mathrm{cm}^{2}\right)$ requires a frame with micro-holes with a maximum diameter of $200 \mu \mathrm{m}$. Hence, we constructed frames containing such micro-holes. However, such small holes require a relatively thin (and strong) support frame that will not be illuminated by the high-intensity laser pulses (incident at 45 degrees), as plasma from the frame may influence the interaction of the laser with the nanofoil itself.

The two different types of frames we fabricated are shown in figure 5.1. Figure 5.2 shows a schematic drawing of the cross-section of the two types of frames. To be able to support the thinnest carbon nanofoil of only $1 \mathrm{~nm}$ thickness, both frames comprise of a $25 \mu \mathrm{m}$ thick aluminium foil (from Goodfellows) in which we fabricated holes of approximately 200 or $300 \mu \mathrm{m}$ diameter ${ }^{1}$. These micro-holes were obtained with laser machining using a XeCl excimer laser from NCLR ${ }^{2}$, a company that closely collaborates with LPNO. This laser provides a near diffraction limited beam and is used for material processing [180]. Each micro-hole was drilled by using 8 consecutive pulses of $9 \mathrm{~mJ}$ and about 200 ns duration. In this way, the crater wall and debris that forms around the hole after ablation was minimized and could be removed by wet etching. The distance between the micro-holes in the first frame was $1.5 \mathrm{~mm}$, and in the second frame they were separated by $6 \mathrm{~mm}$. Such micro-holes with high geometrical quality would be difficult to obtain via, e.g., mechanical drilling.

In order to get flat support frames made of the thin aluminium foils, special tighten-rings were made to stretch the foil. To give strength to the stretched aluminium foil, it was glued onto a thicker frame of $3 \mathrm{~mm}$ stainless steel or $1 \mathrm{~mm}$ hard aluminium, used in the first and second experimental run respectively (see figures 5.1 and 5.2). In the latter case (second frame), an extra top plate is placed on the frame to diminish laser-induced shocks and vibrations through the aluminium foil that would cause damage to the carbon foil. This effect was observed during the first experimental run, where the distance between the holes was only $1.5 \mathrm{~mm}$ and no top plate was used. The advantage of this second frame is that, because this new frame is thin, it allows us to measure the transmitted or re-emitted light behind the foil ${ }^{3}$. Unfortunately, this design required more effort in obtaining flat nanofoils as compared with the first design. The reason and solution for this will be described below. Nevertheless, flat foils as in figure 5.3 (on the right) were obtained with both types of frames.

Inaccurate preparation of the carbon foils on top of the frames leads to broken or wrinkled nanofoils. In order to assure a well-known angle of incidence for

\footnotetext{
${ }^{1}$ The size of the holes are much larger than the focal spot waist of the laser which is less than $10 \mu \mathrm{m}$ FWHM

2 www.nclr.nl, [180]

${ }^{3}$ With the first frame, this was not possible because the frame holder blocked the transmitted beam and the ions coming off the rear surface of the foil that travel in the forward direction.
} 
(a)

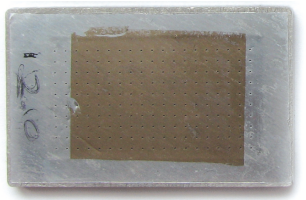

(b)

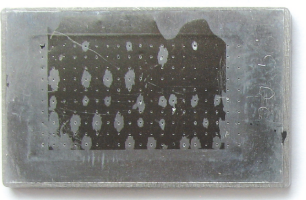

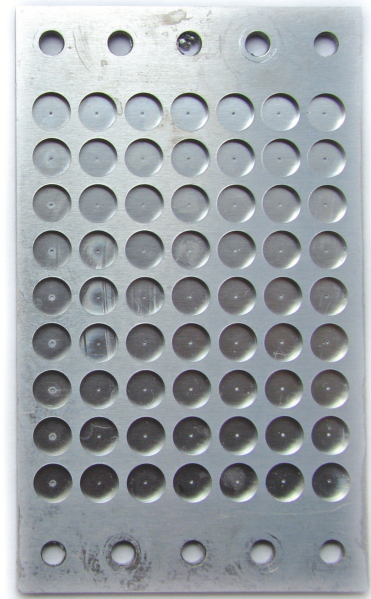

(c)

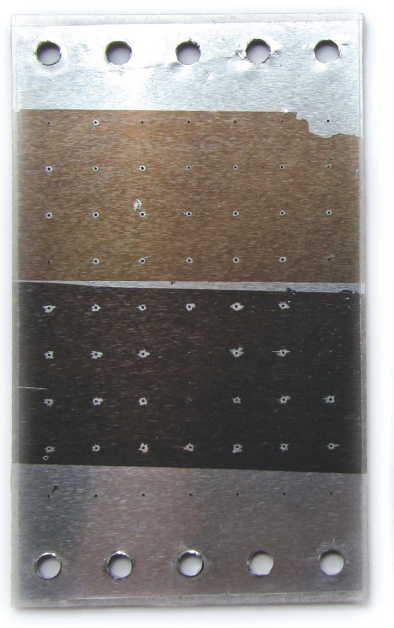

(d)

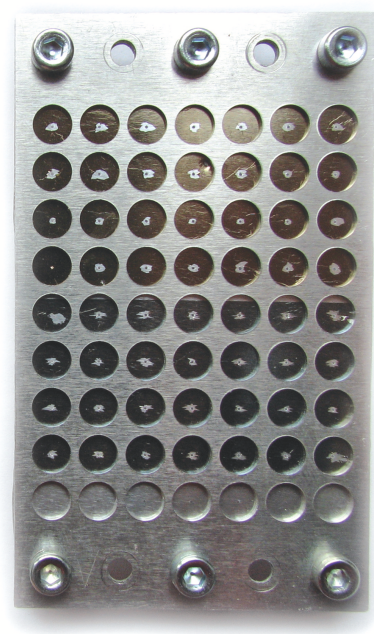

(e)

Figure 5.1: Two different fabricated frames. (a) and (b) show the small $3 \mathrm{~mm}$ thick frames of $2.5 \times 4 \mathrm{~cm}$, supporting the $25 \mu \mathrm{m}$ thick aluminium foil with $200 \mu \mathrm{m}$ holes, which were used for experiments with the UHI 10 laser. The second design of the frame $(4.5 \times 7.6 \mathrm{~cm})$, used for experiments with the UHI 100 laser, is shown in photos (c), (d) and (e). In this case, holes of approximately $300 \mathrm{~nm}$ were drilled with an Excimer laser [180]. On top of the aluminium foil one sees in (a) an unused $10 \mathrm{~nm}$ thick carbon foil and in (b) a $25 \mathrm{~nm}$ carbon foil, which had been irradiated with the UHI 10 laser. It can be seen that the damaged area generated by the laser shots is larger than the micro-hole. Often, the carbon foil that initially covered a neighboring micro-hole was found to be damaged as well. This problem was solved with the second set of frames where the spacing between the micro-holes was enlarged and a top plate was mounted to diminish vibrations. (c) The laser irradiated side of the $1 \mathrm{~mm}$ thick frame supports on the back side the carbon foils (d), in this example 11 and $19 \mathrm{~nm}$ thick. (e) An example of the frame with a top plate. This example holds laser irradiated carbon foils of 71.5 and $35 \mathrm{~nm}$. 


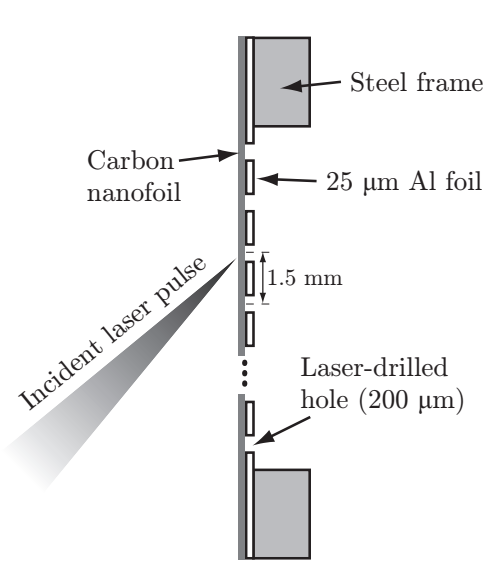

(a)

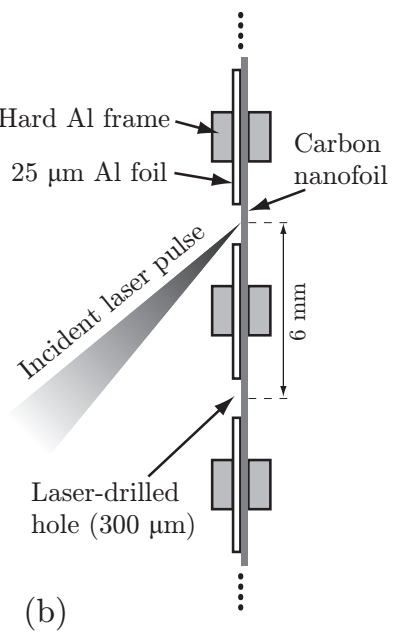

(b)

Figure 5.2: Schematic cross-sectional view (not to scale) of the two types of frames. (a) The frame used during the first experimental run that corresponds to the frames in figure 5.1(a,b). Behind this frame, the frame holder blocks any transmitted light. (b) The frame used during the second set of experiments, which corresponds with the frames in figure 5.1 (c to e).

the laser pulse and of the proton emission and harmonic radiation in the experiments, the described steps of target preparation (floating off and picking up) were optimized to acquire flat carbon foils as confirmed with inspection through a microscope. Examples of a wrinkled foil and a flat foil are shown in figure 5.3 on the left and right, respectively.

The preparation of such flat nanofoils requires a great deal of practice and patience. A photo of the preparation setup is shown in figure 5.4, this is helpful to understand the following description of the preparation procedure. Most steps are carried out according to the technical information provided by ACFMetals [178]. First, in order to match the frame size, the carbon foil is cut to size on the glass substrate before floating off. With a mechanical arm connected to a translation stage, the glass substrate with the carbon film is slowly $(\approx 0.5 \mathrm{~mm} / \mathrm{s})$ lowered into deionized water under an angle of approximately 45 degrees. The carbon foil then smoothly comes off of the glass and floats freely on the water. With the larger frames used during the second run of experiments at PHI, two foils with different thicknesses were subsequently floated off before they simultaneously were picked up.

After floating off the foils, the holder with the substrate is replaced by the holder supporting the aluminium frame, in order to slowly $(\approx 0.1 \mathrm{~mm} / \mathrm{s})$ lift the frame up with the mechanical arm while picking up the foil. The frame has to have a fresh hydrophilic surface [178] and is for this reason acid-etched for 

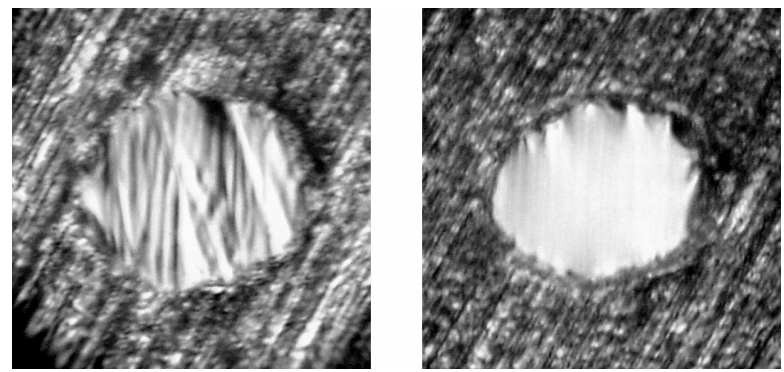

Figure 5.3: Microscope images of carbon nanofoils supported on a first-design frame that contains micro-holes of approximately $200 \mu \mathrm{m}$. The photo on the left shows an example of a wrinkled foil. The photo on the right shows an example of a flat foil for which the preparation procedure has been optimized.

about two hours with, for example, a $2 \%$ acid of $\mathrm{HNO}_{3}$ before picking up the carbon foil.

The free-floating foils are positioned above the frame via slow water currents formed by gently blowing along the water surface with air or nitrogen before being picked up. For the picking up procedure, we found that, to obtain flat foils, there is an optimum angle of the frame surface that depends on the foil thickness; the frame should be held nearly horizontally for foils thinner than approximately $20 \mathrm{~nm}$, while foils thicker than approximately $80 \mathrm{~nm}$ require an almost vertical position of the frame.

After the foils are placed onto the frame they are dried. A problem that arises during the drying process is that water inside the micro-holes does not evaporate gradually but in sudden steps which breaks or wrinkles the nanofoil. With the small frames that contain the closely spaced micro-holes, it was sufficient to break the surface tension of the water by touching the bottom of the wet $\mathrm{Al}$ foil before picking up the nanofoils. During picking up, the water flows smoothly out of the micro-holes. The foils can then be let to dry at room temperature for about 30 minutes. However, in case of the larger frames, the larger spacing between the micro-holes and the circular pattern in the frame causes the water to adhere on the frame, filling the micro-holes and proving difficult to remove. Several methods were examined to solve this problem. We found that heating the prepared samples to 50 to $60{ }^{\circ} \mathrm{C}$ resulted in the flattest carbon foils.

The preparation is done at the location of the laser experiment since the freestanding foils are very fragile and easily broken by shock, vibration, and air currents [178]. The foils can be stored horizontally in a safe environment (dry and free from shocks, vibrations and air currents) for a few days; vertical storage was found to result in wrinkling of the foils. A point of concern is that the interaction chamber where the foils are mounted, needs to be pumped 


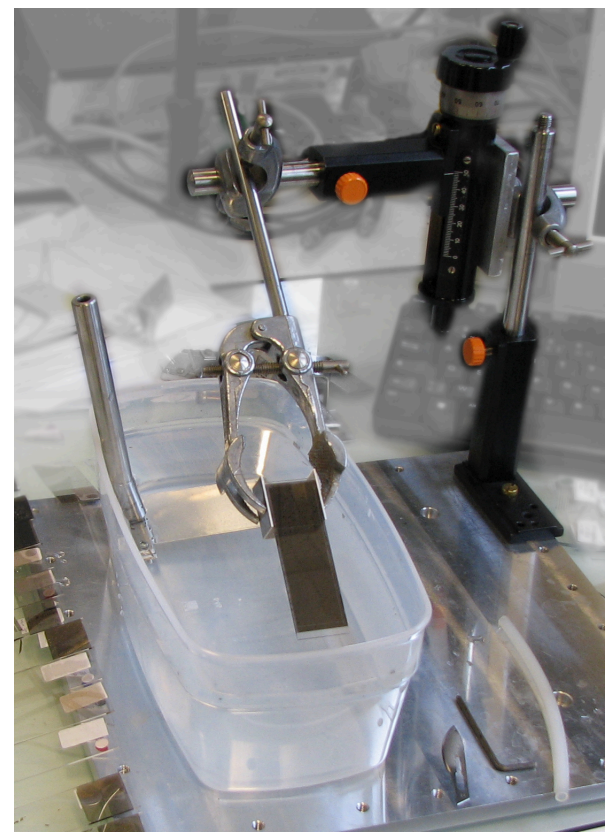

Figure 5.4: The simple but practical setup for preparing the nanofoils onto a frame. The procedure is described in the text. It requires a great deal of practice and patience to obtain very flat foils as shown on the right photo in figure 5.3.

down to vacuum which induces air currents. This process has to be performed slowly (typically a vacuum of $10^{-5}$ mbar was reached in about 30 minutes). An additional advantage of the custom-made frames is that the small micronsized openings covered with the nanofoil makes it slightly less sensitive to air currents, as compared to larger openings. This additional strength to the thin foils makes them easier to handle and helps prevent the foils breaking in the preparation chamber when the vacuum is created.

It should be noted that, due to surface adsorption of carbon hydrogen and water vapor, and the extremely low thickness of the prepared foils, the amount of hydrogen atoms in the prepared foil samples can become substantial, up to 30 at.\% (atomic percent) for the thinnest foils [181]. The consequence of this uncertainty is, e.g., that the effective density of the foil could change. This may also change the amount of laser energy that is converted into the hot electrons responsible for $\mathrm{HOHG}$ and ion acceleration. This can be adequately addressed in theoretical modelling.

As well as the carbon nanofoils, we also used other targets in the experiments, namely ultrathin foils of plastic polystyrene (100 to $800 \mathrm{~nm}$ ) and polyethylene 
terephthalate (PET) (1 to $50 \mu \mathrm{m})$. We also used bulk targets of plastic, silicon, silica and nano-structured multi-layered MoSi. The nano-layered MoSi consists of 50 bi-layers of molybdenum and silicon with a periodicity of $7 \mathrm{~nm}$.

\subsection{Experimental setup}

In order to provide an impression of the experimental conditions during the measurements with both laser configurations, this section describes the experimental setup of the interaction chamber.

The experiments described in this chapter were performed with the UHI 10 and UHI 100 laser at PHI (see section 1.1). The UHI 10 laser was used for the experiments on the coherent dynamics of plasma targets, which is discussed in section 5.3.1, as well as a first experimental run on ion acceleration from extremely thin foils, discussed in section 5.3.2. The UHI 100 laser was used during the second experimental run on ion acceleration while simultaneously the harmonic signal was investigated.

Figure 5.5 shows a schematic drawing of the interaction chamber. The laser pulse (which possesses approximately a flat-top transverse profile of $60 \mathrm{~mm}$ diameter), coming from the compressor, was cleaned from ASE and a pedestal to achieve an ultra-high pulse contrast with the double-plasma mirror (DPM) before entering the main interaction chamber. The pulse contrast after the double plasma mirror on nanosecond time scale was measured to be $10^{10}$ for the UHI 10 laser, and estimated to be $10^{13}$ for the UHI 100 laser (see section 1.1 and appendix A). With an off-axis parabolic mirror (OAP) the pulse is focused on the target in the middle of the chamber in p-polarization under an angle of 45 degrees. The focal spot size is determined by the focal length of the OAP. During the first experimental run with the UHI 10, we used an OAP with a focal length of $500 \mathrm{~mm}$. The estimated peak intensity could be set to two values via circular diaphragms (30 or $40 \mathrm{~mm}$ diameter): $5 \cdot 10^{17} \mathrm{~W} / \mathrm{cm}^{2}$ and $2 \cdot 10^{18} \mathrm{~W} / \mathrm{cm}^{2}$. In addition to the shorter pulses and higher pulse energy of the UHI 100, during the second series a focal length of the OAP of $300 \mathrm{~mm}$ was used to further increase the intensity. Focused intensities of up to $3 \cdot 10^{19} \mathrm{~W} / \mathrm{cm}^{2}$ were estimated. During the second series of experiments, the intensity could be regulated via computer-controlled filters. To obtain multiple focal spots on the target, a piece of frosted glass that contained multiple slits could be placed into the beam path in front of the focusing mirror. The multiple focal spots were used during the experiment described in section 5.3.1.

Two methods of diagnostics were used to investigate the harmonics in the reflected beam. Harmonic spectra were recorded by using a toroidal mirror and an XUV diffraction grating both used at near-grazing incidence [182]. The XUV grating was rotated to select various spectral ranges that contained different harmonic orders. A full spectral measurement requires several shots on target and rotation of the grating in between the shots. To measure the far-field profile of the harmonic beam a second diagnostic instrument is used. It is a removable construction and is shown schematically in the insert of figure 5.5. 


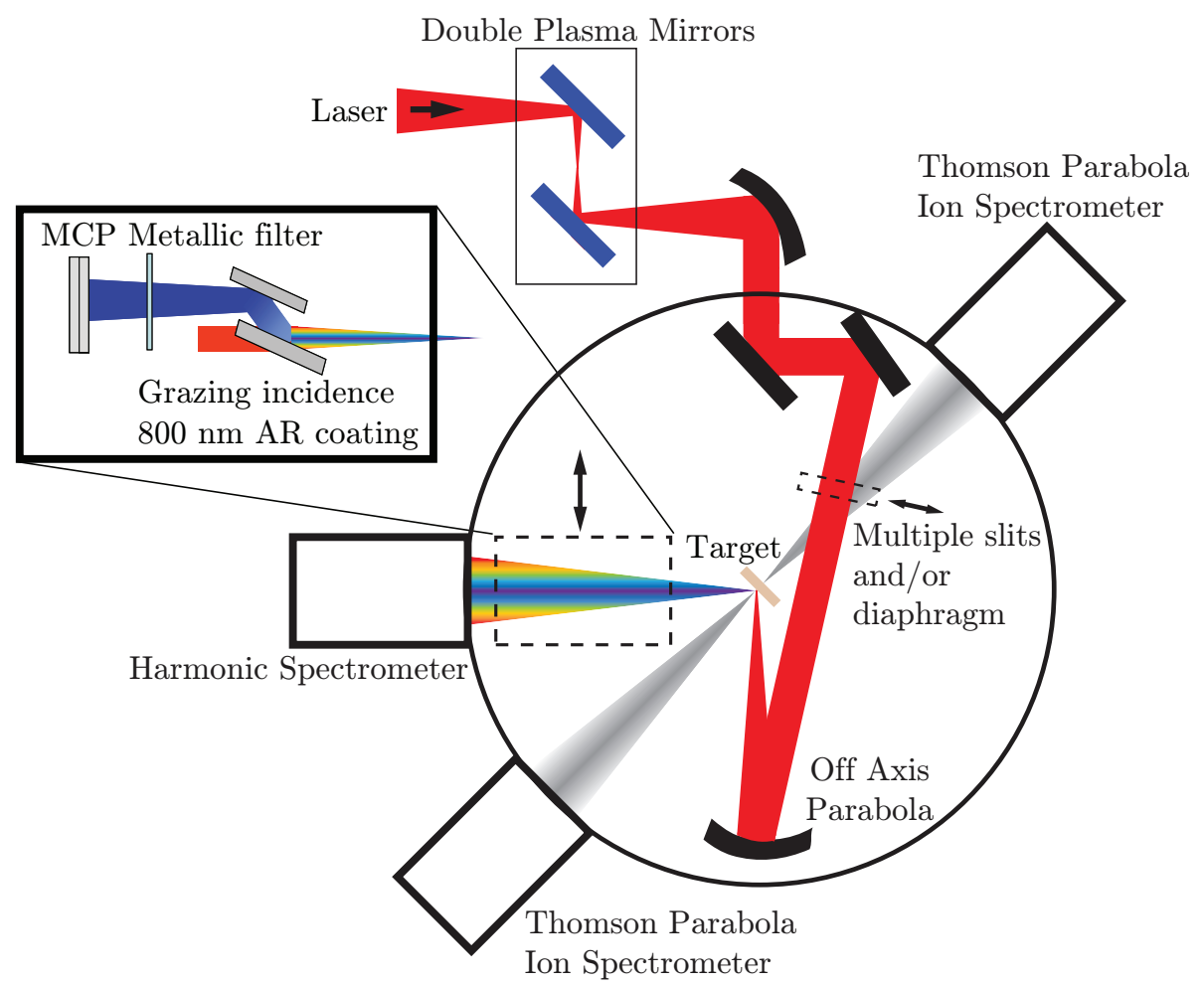

Figure 5.5: Experimental interaction chamber. After the double plasma mirror, the laser pulse from the UHI 10 or UHI 100 laser is focused on the target in the middle of the chamber with an off-axis parabola. The removable diaphragm and multiple slits stand in the beam path in front of the focusing mirror. Harmonic spectra of the reflected pulse are measured with a spectrometer. Alternatively, the spatial profile is measured with an MCP detector after filtering, shown in the insert, which is a removable configuration. Ion spectra are measured normal to the target surfaces with a Thomson parabola ion spectrometer.

It comprises a filter (two IR anti-reflection coated plates and a $200 \mathrm{~nm}$ thick tin or indium foil) that removes the fundamental laser frequency and transmits a few harmonic orders. Double-stage microchannel plates (MCP) coupled to a phosphor screen where fluorescence is imaged on a CCD (charge-coupled device), is used to record the far-field profile. Further details are described in [176]. 
Spectra of the kinetic energy of protons and ions were recorded using Thomson parabola spectrometers $[183]^{4}$ placed normally to both target surfaces. These spectrometers comprise a combination of two permanent magnets and two steel electrode plates driven at a high-voltage of $4 \mathrm{kV}$. The resulting magnetic and electric fields are oriented parallel to each other, such that ions with a different charge-to-mass ratio and different velocities can be recorded, using a double MCP system coupled to a phosphor screen and a CCD camera. On the camera, each charge-to-mass ratio (type of ion) shows as a separate trace with a parabolic shape, with the curvature inversely proportional to the ratio. The coordinate along each trace can be evaluated to obtain the kinetic energy of the corresponding type of ion. During the first run with the UHI 10 laser, only the Thomson parabola spectrometer that measured ions coming from the front surface of the irradiated foils, was installed. During the second run with the UHI 100 a second Thomson parabola spectrometer was installed to also measure the ion energy spectra from the rear surface, however, due to a malfunction, the recorded data could not be used for an evaluation.

During the second experimental run, extra control and reference during the shots was achieved by using two CCD cameras that record the scattering of IR drive laser beam profiles on frosted glass plates in reflection and, if present, in transmission.

\subsection{Experimental results}

In the following, the experimental results as obtained from measurements of HOHG by CWE are described. The results prove, for the first time, the spatial coherence of such harmonics. Harmonic spectra were also recorded from nano-structured MoSi targets and ultrathin foils. Additionally, results of measurements on proton acceleration from ultrathin foils will be described.

\subsubsection{Coherent dynamics of overdense plasmas}

The generation of a highly directional light beam provides a strong indication but not a guarantee that the source is spatially coherent. For example, certain types of partially coherent sources ${ }^{5}$ will generate an optical field in the far-field that has the same intensity distribution as a Gaussian laser beam [53]. Also, incoherent radiation from relativistic electrons can result in collimated beams from synchrotrons for example [61] or an electron bunch accelerated via laser wakefield acceleration guided in a plasma channel [184]. These examples of directional, incoherent radiation indicate that the directional harmonic radiation from the highly complex medium (that is a solid target irradiated with a high-intensity laser pulse) is not necessarily coherent but may be incoherent radiation. The most direct way to determine whether radiation is coherent is

\footnotetext{
${ }^{4}$ http://iramis.cea.fr/spam/MEC/ast_visu.php?lang=ang\&num=210\&keyw $=$ http://iramis.cea.fr/spam/MEC/doc/Presentation_pageweb.pdf

${ }^{5}$ The source is spatially incoherent in a global sense, as named by the authors of [53].
} 
to examine the presence of interference fringes. To experimentally prove that the process of HOHG via CWE results in spatially coherent harmonic radiation we generated high-order harmonics with three phase-related sources and verified the existence of interference fringes in the far-field spatial profile. Here we give a description of the main results, and full details can be found in our publication [176].

The interference fringes also provide more detailed information on the spectral phase of the harmonics and give insight into the dynamics of the plasma electrons responsible for HOHG on the attosecond time scale. Theoretically this should imply variations in the dynamics of the system and hence in the temporal properties of the radiated harmonic field. In particular, there should be an intensity-dependence of the phase of individual harmonics (see section 3.3.2). Accordingly, the interference pattern should change when the intensity ratio between the sources is altered.

To obtain three temporally synchronized sources of HOHG, we placed a transmission grating (a piece of frosted glass that contains multiple slits) into the laser beam in front of the focusing mirror, as is indicated in figure 5.6a, in such a way that a central spot surrounded by two slightly weaker satellite spots is produced at the focus by diffraction. The intensity ratio between the central spot and satellite spots depends on the grating periodicity and the width of the slits. The grating periodicity also influences the distance between the spots. Primarily we used a grating with a periodicity of $1 \mathrm{~cm}$ and slits of $4 \mathrm{~mm}$ wide. This led to a distance of $40 \mu \mathrm{m}$ between the spots and an intensity ratio of 0.57 . In this experiment with the UHI 10 laser, we focused 60 fs laser pulses with an ultra-high temporal contrast $\left(10^{10}\right)$ on bulk silica to intensities of the central spot from a few times $10^{16} \mathrm{~W} / \mathrm{cm}^{2}$ to a few times $10^{17} \mathrm{~W} / \mathrm{cm}^{2}$. With such intensities it can be expected that the high-order harmonics are generated via the process of CWE, rather than ROM.

Figures 5.6b and c (blue curve) show, respectively, a single-shot spatial profile and its lineout in the far-field that contains fringes with a high contrast of about $80 \%$ in the center of the pattern. These fringes result from the interferences of the three spatially separated XUV sources. The total signal is the superposition (sum of intensities) of the interferograms corresponding to each harmonic frequency. The presence of these interference fringes demonstrates the mutual coherence between the three harmonic sources.

Assuming that, at the applied laser intensities, CWE is responsible for the generation of high-order harmonics, does the emitted light indeed follow the theoretically predicted dependence on intensity, i.e., is the current physical model of CWE complete? As mentioned before, interferograms such as in figure 5.6b, provide more detailed information on the phase of the harmonics and give insight into the dynamics of the plasma electrons responsible for HOHG. We have extracted such information from the spatial Fourier transform of the interferogram, an example of which is shown in figure 5.7, where a tin filter was placed in the beam that transmits the $10^{\text {th }}$ to $15^{\text {th }}$ harmonic orders. In the next paragraphs we describe the interpretation of this data in 

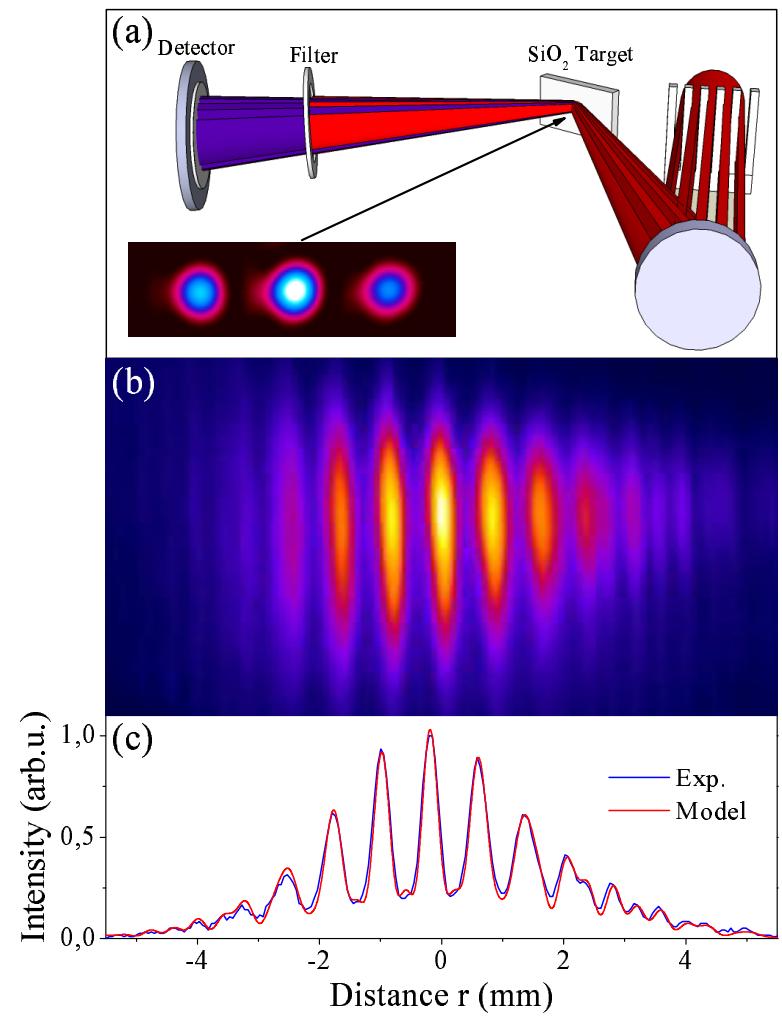

Figure 5.6: Coherent harmonic beams from plasma mirrors by CWE. (a) Experimental setup and intensity distribution in the focal plane for an intensity ratio between the central spot and outer spots of 0.57 . (b) Single-shot interference pattern of harmonics 8 to 10 (selected using a $200 \mathrm{~nm}$ thick indium filter) in the far-field. (c) Lineout of the fringes (blue curve) and a theoretical fit (red curve).

more detail, for a full quantitative analysis the reader is referred to our article in [176].

With CWE it is predicted that a different drive laser intensity would induce a change in the phase of the emitted harmonics (see section 3.3.2). This means that there should be a phase shift between the harmonics generated from the central source and the harmonics generated from one of the outer sources. For each harmonic frequency in the spectrum, the spatial Fourier transform contains information about the interference of the central source with one of the satellite sources, corresponding to a spot indicated with $S^{1}(\mathbf{q})$ in figure 5.7, and about the interference between the two outer sources, corresponding to $S^{2}(\mathbf{q})$. The ratio of these two terms $\left(S^{1}(\mathbf{q}) / S^{2}(\mathbf{q})\right)$ thereby encodes the phase 


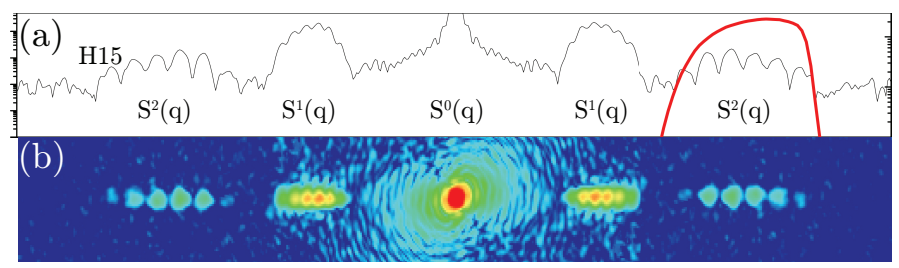

Figure 5.7: Spectra $S(\mathbf{q})$ in log scale of a single-shot interferogram where the harmonics 10 to 15 were selected using a $200 \mathrm{~nm}$ thick tin filter. (a) shows a lineout of the spatial Fourier transform (b). The spot at $S^{1}(\mathbf{q})$ corresponds to interference between the central spot and the satellite sources. The spot at $S^{2}(\mathbf{q})$ corresponds to interference between the two satellite sources. The transmission curve of the used filter is shown as a red line around the $S^{2}(\mathbf{q})$ term.

shift between the central harmonic source and the two lateral ones. An altered phase shift affects this ratio and also the contrast of the fringes in the spatial profile.

Because in CWE the phase of the harmonics is correlated to the time of emission, the phase shift of the harmonics between the central and outer sources should be linearly related to a delay in the emission time. This is in accordance with numerical calculations with PIC codes [168] and is due to the different return time of the Brunel electrons as the laser intensity is altered (see section 3.1.3). Complete analysis of the spectrum and its Fourier transform, as described in detail in [176], enables us to extract the values of these delays, which are smaller than 100 attosecond. The analysis also allows us to theoretically reconstruct the fringe pattern created by the interference of the harmonic emission of the central source with the delayed emission of the two outer sources. As an example, the result of such a theoretical fringe pattern is displayed in figure $5.6 \mathrm{c}$, for comparison with the experimental data. It can be seen that the model perfectly fits the experimental data.

From this one can conclude that the observed harmonics are generated by CWE. A second conclusion is that the phase of the generated harmonics can be controlled via the drive intensity as theoretically predicted and, that this phase control can reach extremely high precision, corresponding to a temporal control of the plasma electrons on attosecond time scales.

Another experiment on $\mathrm{HOHG}$ was performed with nano-structured multilayered targets. Multi-layer mirrors that consist of alternating layers of high and low refractive index material with a certain periodicity enhance the reflectivity of light with a wavelength of twice the periodicity of the layers. This occurs via the constructive interference from light reflected at the different 
layers. An example of such a mirror applicable in the XUV range is nanostructured multi-layered MoSi [185]. We considered if a similar effect could be employed in a periodically nano-patterned plasma with alternating layers of high and low electron density such that constructive interference of HOHG from these layers would result in an enhanced generation efficiency. To obtain such a nano-structured plasma, a neutral multi-layer stack of high and low Z-number materials, such as Mo and Si, with layers of a few nanometers should be instantaneously ionized to some depth with ultra-high contrast, high-intensity laser pulses.

Initial results of harmonic spectra obtained from such a nano-structured multi-layered target are shown in figure 5.8, with the spectrum from MoSi on the left and for comparison a spectrum from a non-structured (only $\mathrm{Si}$ ) target on the right. The spectra are assembled from subspectra of a narrow range in order to provide a wider spectral overview. Also, the spectra are compensated for the gain of the camera and matched to the zero-order angle of the spectrometer in order to enable the matching of the subspectra and a better quantitative comparison.
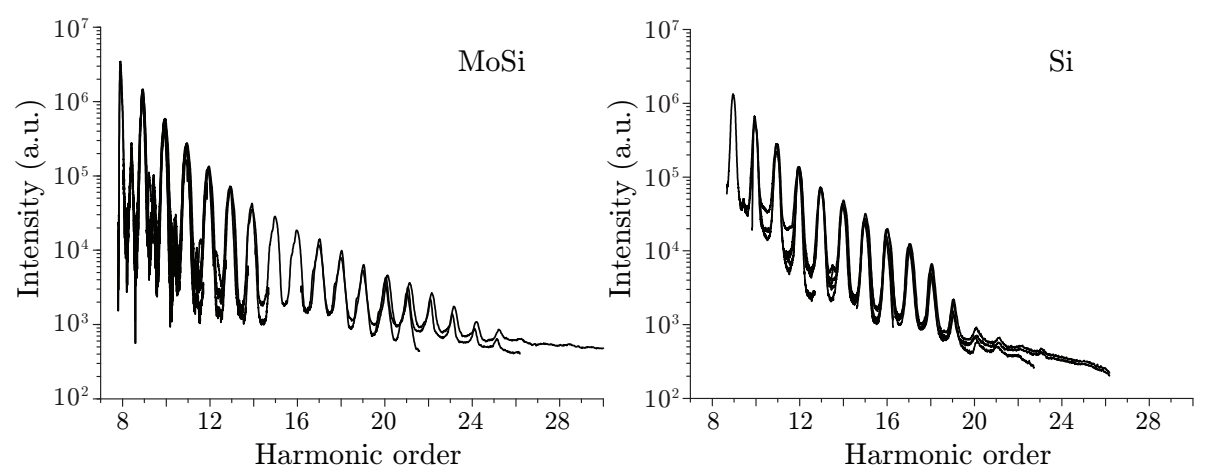

Figure 5.8: CWE spectra of nano-structured multi-layered MoSi (left) and silicon (right). Up to the $17^{\text {th }}$ order the spectra exactly overlap. Both spectra consist of multiple subspectra that correspond to several laser shots on target, as explained in section 5.2.

As can be seen from the graphs in figure 5.8, the spectra from both MoSi and $\mathrm{Si}$ are identical up to the $17^{\text {th }}$ harmonic order, while at higher orders there is a clear difference. Here the MoSi sample exhibits harmonics towards higher orders $\left(25^{\text {th }}\right)$ than the Si sample $\left(20^{\text {th }}\right)$. A possible explanation for this observation follows. Assuming that the harmonics are generated via CWE (justified by the used laser intensity of about $10^{18} \mathrm{~W} / \mathrm{cm}^{2}$ and the observations reported in the previous section) then, due to the basic working of CWE (see section 3.3.2), the highest expected harmonic order would be given by the maximum plasma frequency, scaled with the Z-number of the used materials and 
their degree of ionization. Indeed, we found that the maximum harmonic order of 20 for Si corresponds to the maximum electron density of fully ionized Si. (For an overview of achievable electron densities with different materials, see appendix D.) Fully ionized pure Mo would enable a maximum harmonic order of 39 , however the observed maximum harmonic order of 25 is somewhat lower. This is consistent with our expectation that Mo would not be fully ionized at the used laser intensity. Looking at these consistencies, there is no apparent effect of the nano-structure on the electron dynamics. Instead, what has been observed seems simply to be the result of an average increase of the electron density in the MoSi sample in comparison with the Si sample. Such averaging, presumably, is caused by the ionized nano-layers being so thin that they distribute homogeneously in the plasma during the expansion. The maximum harmonic order of 25 would correspond to the maximum homogenized plasma density of fully ionized Si and partly ionized Mo.

To preserve a nano-patterned plasma, it might be worth trying other samples, for example, with thicker layers, possibly with different materials (lower Z-number to increase the penetration depth) and use pulses with a higher contrast to reduce the pre-expansion-induced homogenization.

\subsubsection{Proton acceleration from nanofoils}

Numerical calculations predict an enhancement of the flux and maximum energy of accelerated ions when irradiating high-intensity laser pulses onto ultrathin foils in the so-called transparent regime. In order to test the validity of this prediction, we experimentally investigated ion acceleration in this transparent regime for the first time using extremely thin targets (nanofoils) and ultra-high contrast, high-intensity laser pulses. We performed two sets of experiments on nanofoils, the preparation of which was described in section 5.1. Besides the described nanofoils from carbon, we also used much thicker foils, between $100 \mathrm{~nm}$ and $50 \mu \mathrm{m}$, of PET (polyethylene terephthalate) and a polystyrene monopolymer plastic. The results from proton acceleration of the first experimental run with the UHI 10 laser are described next. Further detail can be found in our article [186]. A description of the results of the second experimental run, with the UHI 100 laser, is given here as well [187].

During the first experiments, the sample construction allowed us to measure ions only in the backward direction. Also the small frames and closely spaced micro-holes allowed us to record results of only a few representative laser shots per sample, due to problems with shocks and vibrations (see section 5.1). Nevertheless, for each foil thickness and two intensities $\left(5 \cdot 10^{17} \mathrm{~W} / \mathrm{cm}^{2}\right.$ and $\left.2 \cdot 10^{18} \mathrm{~W} / \mathrm{cm}^{2}\right)$, a set of spectra was recorded with several laser shots. Each set of spectra was analyzed for the maximum proton energy, using the commercial code SIMION [133]. In figure 5.9, the maximum proton energy in each set of spectra is shown as a function of the foil thickness and for the two applied laser intensities (square and triangular symbols for the lower and higher intensity, respectively). 


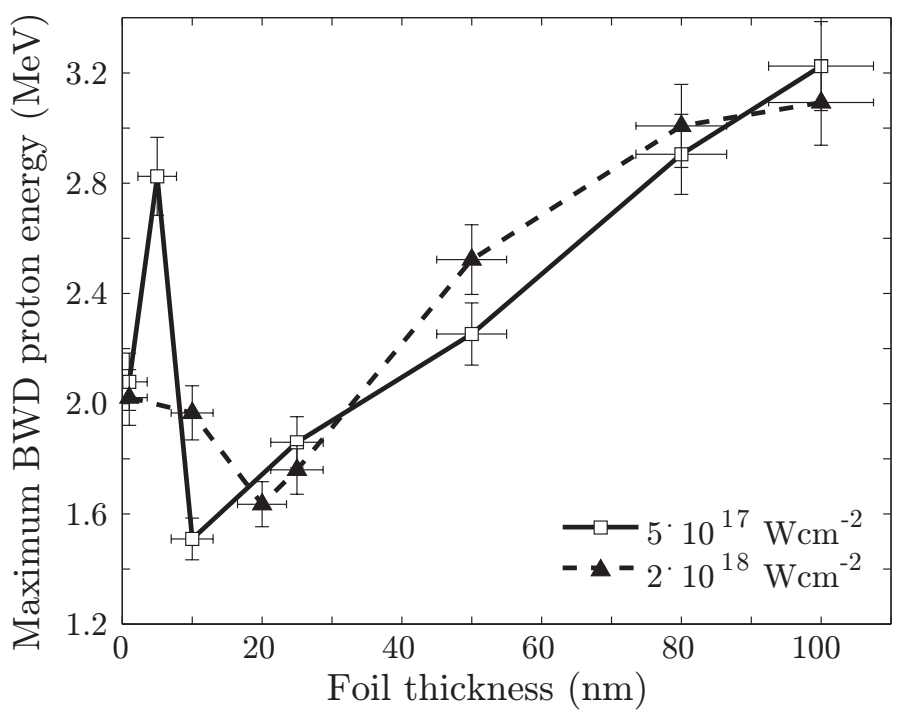

Figure 5.9: Maximum proton energy observed in the backward direction (BWD) normal to the target, using two different light intensities for the ultra-high contrast $\left(10^{10}\right)$, ultrashort (65 fs) laser pulses of UHI 10. The square symbols (connected by a solid line) correspond to a laser intensity of $5 \cdot 10^{17} \mathrm{~W} / \mathrm{cm}^{2}$ and the triangles (dashed line) correspond to $2 \cdot 10^{18} \mathrm{~W} / \mathrm{cm}^{2}$. The lines are guides for the eye.

Surprisingly, in contrast to what was predicted, one can identify an enhanced kinetic energy of protons accelerated in the backward direction achieved with the extremely thin carbon foils, i.e. in the range of 5 to $20 \mathrm{~nm}$ thickness. For comparison, these foils are not much thicker than the penetration depth of the laser light, considering that the used pulses with their ultra-high contrast limit the pre-expansion of the plasma and, thus preserve a high plasma density (see section 3.2.2). Note that this regime has never been investigated before because in previously performed experiments with ultra-high contrast pulses, the foil thicknesses were restricted to values above $20 \mathrm{~nm}$, as described in section 3.2.2. In those experiments, an optimum foil thickness had been found in the range of 50 to $100 \mathrm{~nm}$. It was expected that below these values, the maximum proton energy would simply decrease towards zero with the decreasing thickness due to a stronger expansion and a larger density scale length.

When comparing those findings in the range of larger foil thicknesses (20 to $100 \mathrm{~nm}$ ) with our own experimental data, it can be seen that our maximum proton energy also decreases with the foil thickness when restricted solely to the 20 to $100 \mathrm{~nm}$ range. This follows the trend suggested by previous experiments with thicker foils that showed an optimum for the maximum proton energy 
around $100 \mathrm{~nm}[131,133]$ and is consistent with the expectation that, due to the ultra-high pulse contrast, the plasma expansion from the front surface extends only over a small range as limited by the pulse duration. From this it would be expected that the expansion would become increasingly detrimental for further decreasing foils thickness.

However, further decreasing the foil thickness towards the penetration depth of the laser light (below $20 \mathrm{~nm}$ ), increases the maximum proton and ion energy. Only towards much lower thicknesses than expected from previous experiments and models (in the range of $1 \mathrm{~nm}$ ) does our data show that the energy decreases. The similarity of proton energies for the two different intensities in the range of 20 to $100 \mathrm{~nm}$ could be the result of an increased electron heating with increasing intensity that is compensated by an increased scale length. What can further be seen in figure 5.9 is that, with decreasing thickness, the observed onset of re-increased energy occurs at a larger thickness when the laser intensity is higher. The latter observation could be the result of a deeper penetration of the laser pulse, for example, due to a lowering of the effective plasma frequency at higher intensities (relativistic transparency, see section 2.1) or to a faster plasma expansion and subsequently lower maximum plasma density.

A similar re-increase of maximum proton energy towards lower thickness was observed, with an optimum thickness at about $50 \mathrm{~nm}$, in the forward direction by Fuchs et al. [188]. In those experiments, however, thicker foils, longer pulses and a lower pulse contrast were used. The explanation for this observation was that the laser interacted with partially blown-out plasma of which the density was still high enough to accelerate a substantial number of hot electrons.

In a joint paper [189] with experimental researchers from PHI and LPNO, Andreev (from the STC Vavilov State Optical Institute in Russia) presented an analytical model that is able to correctly predict the standard maximum of the proton energy observed in the experiments at PHI by others [133] and us [186], provided that the foil thickness is restricted to values well above $25 \mathrm{~nm}$. However, the additional maximum at extremely thin foils could not be explained by this model because such low thicknesses, in the order of a few nanometers, fall out of the applicable range of that model.

Therefore, we aimed to interpret of our experimental results with our own extension of a numerical modeling, which will be discussed in section 5.4. The discussion will point out that the effect is largely dependent on the laser pulse parameters and the plasma density gradient.

In the second experimental run with the UHI 100 laser, a new set of experiments was performed on the nanofoils as an extension and confirmation of the results of the first run. The pulses available in the second run were shorter ( $25 \mathrm{fs}$ ), had a higher peak intensity $\left(3 \cdot 10^{19} \mathrm{~W} / \mathrm{cm}^{2}\right)$ and, a higher temporal temporal contrast $\left(10^{13}\right)$. Also, when compared to the first run, more shots on foil targets could be executed and thus more data on the accelerated ions could be recorded in both the backward and forward direction. This was due to the 
improved second sample mounting as was described in section 5.1. However, at the end of the run, it appeared that ion spectra recorded in the forward direction seemed partly inconsistent upon further analysis (with the SIMION code). A possible explanation could be that the Thomson parabola spectrometer used in the forward direction was malfunctioning due to electromagnetic pulses (EMP) that are induced when the ultrashort laser pulse interacts with the target.

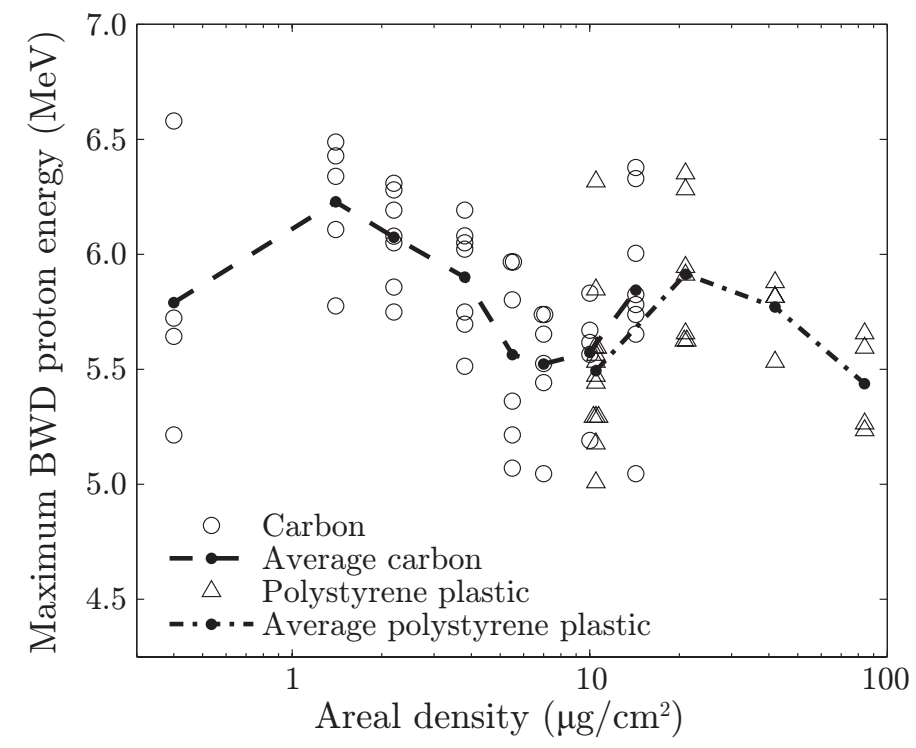

Figure 5.10: Maximum proton energy observed in the backward direction (BWD) normal to the target, using a light intensity of $3 \cdot 10^{19} \mathrm{~W} / \mathrm{cm}^{2}$ for the ultra-high contrast $\left(10^{13}\right)$, ultrashort (25 fs) laser pulses of UHI 100. The open symbols are measured data points, with an experimental error of less than $0.5 \mathrm{MeV}$ (not shown in the graph for better readability). At each foil thickness, the filled dot is the average value over all shots.

Nevertheless, the ion spectra from the Thomson parabola recorded with the other drive electronics in the backward direction appeared reliable and consistent, this is probably due to better shielding from EMPs. In figure 5.10, the measured maximum proton energy for polystyrene mono-polymer plastic and carbon foils is plotted as a function of the foil areal density for each shot (open circle and triangle symbols). The average of all shots for each foil thickness (filled dotted symbol) is connected with a line for better visibility. Certain data points overlap and are slightly shifted in areal density for a better display of separate data points. All shots are performed with a laser energy of $2.5 \mathrm{~J}$, except for two foil thicknesses (11 and $19 \mathrm{~nm}$ ) where the laser energy is $2.0 \mathrm{~J}$ due to a malfunctioning pump laser. To attempt to account for the reduced pulse 
energy, we plotted (only for 11 and $19 \mathrm{~nm}$ ) data gained from an extrapolation (see appendix E).

To compare the data between the carbon and polystyrene foils, the maximum proton energy is plotted versus the areal density. The areal density contains information about the foil thickness and the electron density. Carbon nanofoils with an areal density of 0.4 to $14.3 \mu \mathrm{g} / \mathrm{cm}^{2}$ have been used, corresponding to thicknesses of, successively, 2, 7, 11, 19, 27.5, 35, 50 and $71.5 \mathrm{~nm}$. Plastic polystyrene foils of 100, 200, 400 and $800 \mathrm{~nm}$ thick have been irradiated with the laser pulses as well. The mass density of these foils is $1.05 \mathrm{~g} / \mathrm{cm}^{3}$ (see also appendix D). As can be seen in figure 5.10, the average values of the maximum proton energy at $10 \mu \mathrm{g} / \mathrm{cm}^{2}$ for carbon and polystyrene are very similar.

When inspecting the general trend of the data in figure 5.10, it can be seen that there is a shot-to-shot fluctuation of more than $10 \%$ in the data on the maximum proton energy. This suggests that the value for the maximum proton energy, as a function of the foil thickness is more or less constant, with a value of approximately $5.8 \pm 0.8 \mathrm{MeV}$. When inspecting the trend of the data in more detail, it can be seen that towards thinner foils there is an increase in the maximum proton energy with decreasing foil thickness down to the first peak at around $20 \mu \mathrm{g} / \mathrm{cm}^{2}$. This can be explained by the enhancement of the electron temperature due to recirculation of the electrons, as described in section 3.2.2. Below $20 \mu \mathrm{g} / \mathrm{cm}^{2}$ the maximum energy drops. We interpret this as the result of a shock wave, induced by the pedestal of the laser pulse, that causes the expansion of the complete foil before the main pulse arrives, as described in section 3.2.2. This trend appears to persist down to an areal density of $7 \mu \mathrm{g} / \mathrm{cm}^{2}$, corresponding to about $35 \mathrm{~nm}$ thick carbon. This decreasing trend for the maximum proton energy versus the foil thickness is comparable to the results obtained with the UHI 10 laser shown in figure 5.9 for the range of 20 to $100 \mathrm{~nm}\left(4\right.$ to $\left.20 \mu \mathrm{g} / \mathrm{cm}^{2}\right)$.

Identical to the previous results with UHI 10, one can, again, observe a second peak in the maximum proton energy at extremely thin carbon foils of a few nanometers which is the range around a surface density of $1 \mu \mathrm{g} / \mathrm{cm}^{2}$. However, in this experiment, with the higher laser intensity of UHI 100, the second peak at a small foil thickness has a higher value for the maximum proton energy than the first peak at thicker foils. In addition, compared with the UHI 10 results, the onset of the re-increase (minimum in between the two peaks) starts at a thicker foil thickness (35 nm thick carbon foil). This is in agreement with the observed difference with the UHI 10 that the onset starts at a higher foil thickness when a higher laser intensity is used.

When comparing the experimental results with the theoretically predicted curve for ion acceleration in the transparent regime with step-like plasma density profiles (see section 3.2.3), both depictions of the experimental trend (double peak or constant value) of the dependency of the maximum proton energy on the foil thickness are not predicted by the theoretical model. In other words, the novel experimental data that we obtained in the transparent regime points 
out that a better physical picture and, subsequently, an improved theoretical description, is required. In order to interpret our experimental results, we performed numerical calculations which will be discussed in section 5.4.

\subsubsection{Harmonic generation from nanofoils}

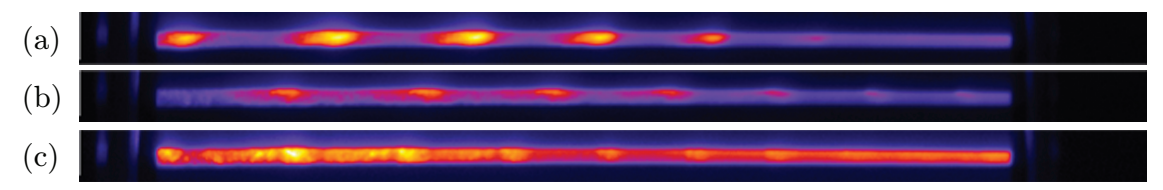

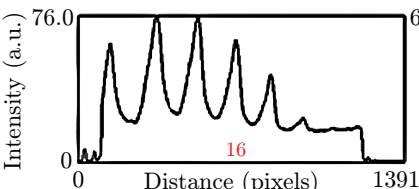

(d)

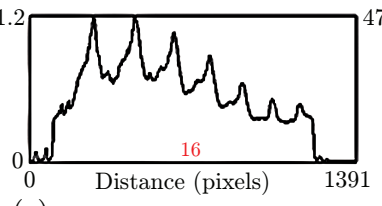

(e)

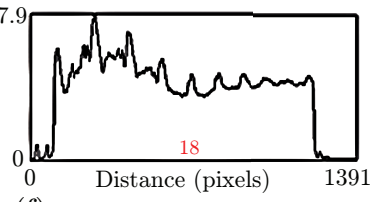

(f)

Figure 5.11: Recorded harmonic spectra (a) to (c) in false-color and the respective power spectra (d) to (f) from bulk silica $\left(\mathrm{SiO}_{2}\right)$, under seemingly identical experimental conditions $\left(I_{0}=3 \cdot 10^{19} \mathrm{~W} / \mathrm{cm}^{2}\right)$. In (a) plus (d) only harmonics due to CWE are observable up to the $18^{\text {th }}$ order. In (b) plus (e) and (c) plus (f) harmonics generated via ROM can also be identified (see main text). The red number indicates the harmonic order at the center of the spectrum (see main text). Compared to (a) and the central harmonic order (red number), the spectrum in figure (b) is somewhat shifted in frequency.

During the experimental runs with the UHI 10 laser and the UHI 100 laser, HOHG spectra were recorded simultaneously with the ion spectra. Additionally, in order to obtain an indication of the presence of any transmitted or re-emitted portion of the infrared beam from the rear surface of the foil, during the measurements with the UHI 100 laser and the carbon nanofoils, a CCD camera recorded the scattering of the laser beam off a frosted glass plate behind the foil in the propagation direction of the laser. No strong harmonic signals were detected from the carbon nanofoils using the UHI 10 laser (up to $2 \cdot 10^{18} \mathrm{~W} / \mathrm{cm}^{2}$ ), however, with the higher intensities (up to $3 \cdot 10^{19} \mathrm{~W} / \mathrm{cm}^{2}$ ) from the UHI 100 laser, harmonics were observed with carbon nanofoils as thin as $7 \mathrm{~nm}$. Harmonics also from various other bulk and foil targets were also recorded for comparison.

In figures 5.11 to 5.16, examples of the recorded spectra are shown for the six different targets investigated. These are, respectively, bulk silica $\left(\mathrm{SiO}_{2}\right)$, bulk plastic, $1.5 \mu \mathrm{m}$ thick PET (polyethylene terephthalate) and carbon nanofoils 


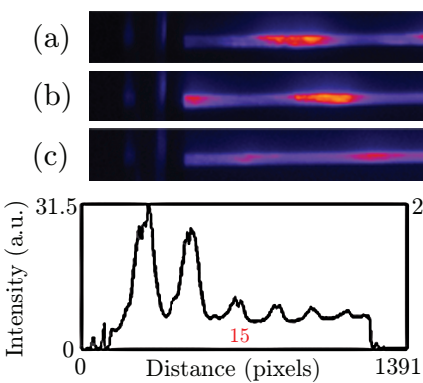

(d)

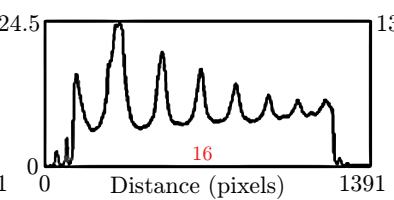

(e)

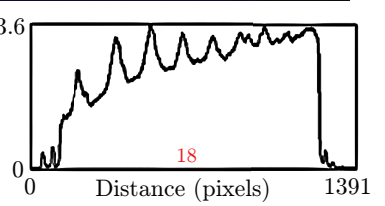

(f)

Figure 5.12: Recorded harmonic spectra (a) to (c) in false-color and the respective power spectra (d) to (f) from bulk plastic, under seemingly identical experimental conditions $\left(I_{0}=3 \cdot 10^{19} \mathrm{~W} / \mathrm{cm}^{2}\right)$. In (a) plus (d) only harmonics due to CWE are observable up to the $14^{\text {th }}$ order. In (b) plus (e) and (c) plus (f) harmonics generated via ROM can also be identified (see main text). The red number indicates the harmonic order at the center of the spectrum (see main text).

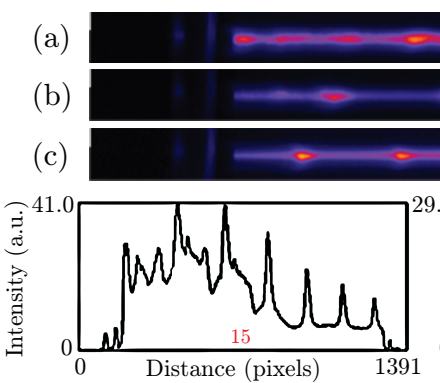

(d)

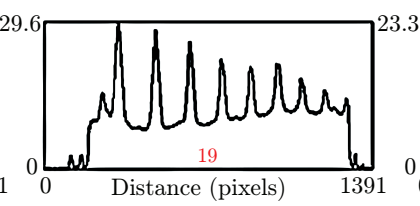

(e)

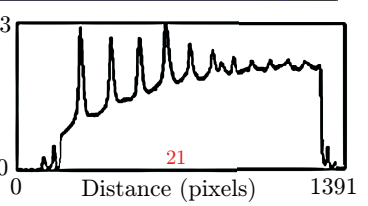

(f)

Figure 5.13: Recorded harmonic spectra (a) to (c) in false-color and the respective power spectra (d) to (f) from a $1.5 \mu \mathrm{m}$ thick PET foil, under seemingly identical experimental conditions $\left(I_{0}=3 \cdot 10^{19} \mathrm{~W} / \mathrm{cm}^{2}\right)$. Spectra show strong harmonics due to CWE up to the $15^{\text {th }}$. Some spectra show strong harmonics due to ROM (up to the $27^{\text {th }}$ order). The red number indicates the harmonic order at the center of the spectrum (see main text).

of 100, 19 and $7 \mathrm{~nm}$ thickness. Harmonic spectra are shown in false-color in the top three images (a) to (c) in each figure. An outline profile of these three images are shown in the lower three plots (d) to (f), respectively. In these 


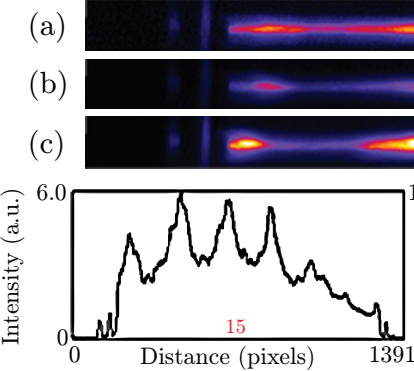

(d)

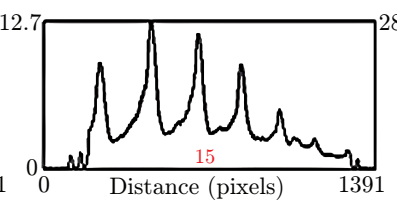

(e)

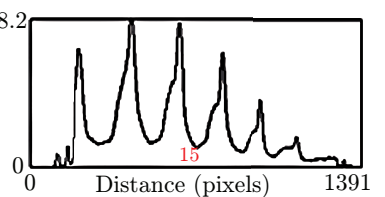

(f)

Figure 5.14: Recorded harmonic spectra (a) to (c) in false-color and the respective power spectra (d) to (f) from a $100 \mathrm{~nm}$ thick carbon foil. Figures (a) plus (d) and (b) plus (e) are spectra when the target was placed in the focus of the laser beam. The harmonic signal is stronger when the target is placed out of focus (c) plus (f), in this case $0.2 \mathrm{~mm}$. The spectrum in figure (a) is enhanced in contrast to make the harmonic signal visible. The red number indicates the harmonic order at the center of the spectrum (see main text).

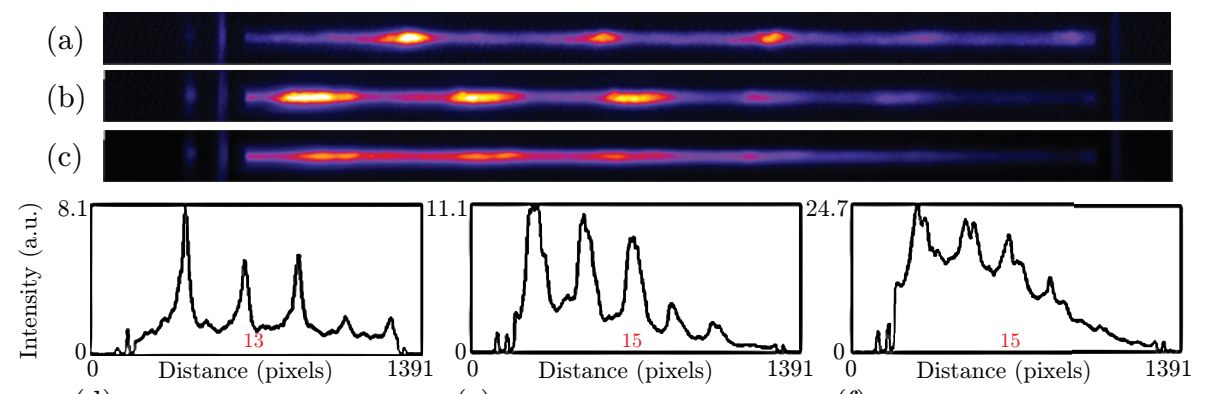

(d)

(e)

(f)

Figure 5.15: Recorded harmonic spectra (a) to (c) in false-color and the respective power spectra (d) to (f) from a $19 \mathrm{~nm}$ thick carbon foil. Figures (a) plus (d) and (b) plus (e) are spectra when the target was placed in the focus of the laser beam. The harmonic signal is stronger when the target is placed out of focus (c) plus (f), in this case $0.45 \mathrm{~mm}$. The red number indicates the harmonic order at the center of the spectrum (see main text).

profiles, the red number corresponds to the harmonic order that is adjusted with the rotation angle of the spectrometer to the central part of the shown 


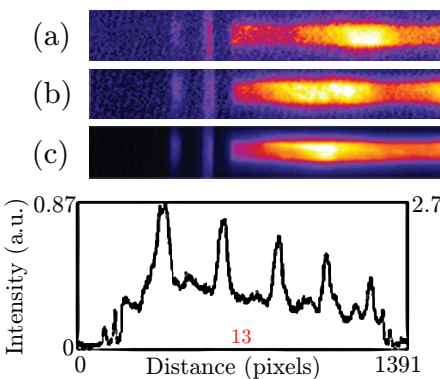

(d) (e)

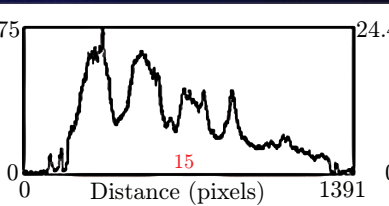

Figure 5.16: Recorded harmonic spectra (a) to (c) in false-color and the respective power spectra (d) to (f) from a $7 \mathrm{~nm}$ thick carbon foil. Figures (a) plus (d) and (b) plus (e) are spectra when the target was placed in the focus of the laser beam. The harmonic signal is stronger when the target is placed out of focus (c) plus (f), in this case $0.45 \mathrm{~mm}$. The spectra in figures (a) and (b) are enhanced in contrast to make the harmonic signal visible. The red number indicates the harmonic order at the center of the spectrum (see main text).

spectral range, this can be used to identify the other integer harmonic orders, particularly the maximum observable harmonic order in the spectral range of the recorded spectrum. In some spectra, the harmonic orders are shifted with respect to the red number, this indicates a small shift in the frequency of these harmonics. The false-color spectra are recorded with different gain factors of the camera. In order to compare the power spectra of figures 5.11 to 5.16 with each other, the vertical scale of the power spectra (figures d to f) has been divided by these gain factors.

In the recorded spectra, high-order harmonics can be attributed to both coherent wake emission (CWE) and the relativistic oscillating mirror (ROM). Indeed, HOHG via ROM is expected to show up since the used intensity of $3 \cdot 10^{19} \mathrm{~W} / \mathrm{cm}^{2}$ should be sufficiently high, based on theoretical predictions and reported experimental results (see section 3.3.1). However, most ${ }^{6}$ of the recorded spectra contained only broad harmonic peaks with an observed maximum order as limited by the plasma frequency of a fully ionized target. These broad harmonics can be ascribed to CWE (see section 3.3.2 and figure 3.7). To give an indication of the maximum generated harmonic order that can be expected due to CWE, the density of the used materials and the corresponding plasma frequency is given in appendix D. An example of a spectrum that contains only CWE is shown in figure 5.11(a) plus (d) from $\mathrm{SiO}_{2}$. On the contrary, only a few recorded spectra contain the typical narrow harmonic orders that

\footnotetext{
${ }^{6}$ Roughly estimated to be more than $90 \%$ by comparing all recorded shots.
} 
can be ascribed to the relativistic oscillating mirror (ROM, see section 3.3.1 and figure 3.7). Examples of such spectra are shown in figures 5.11(b) and (c), 5.12(b) and (c) and 5.13(a) to (c). Despite the higher intensity achieved with the upgraded UHI 100 laser, the maximum observed harmonic order with the bulk targets was similar to the previously obtained spectra by the PHI group with the UHI 10 laser. A possible explanation for this will be given below.

What can further be seen, by comparing the figures 5.11 to 5.16 and our other obtained data, is that the strongest harmonic signal due to CWE was obtained with the silica targets. The strongest and highest order harmonics due to ROM were recorded using $1.5 \mu \mathrm{m}$ PET foils. In the few cases that high-order harmonics were generated due to ROM with the silica and PET targets there is a small harmonic peak from ROM visible on top of the broader harmonic peak due to CWE, up to the $18^{\text {th }}$ and $15^{\text {th }}$ order, respectively, which corresponds to the maximum plasma density of silica and PET. Examples can be seen in figures 5.11(c) plus (f) and 5.13(a) plus (d). Above these maximum harmonic orders for CWE, harmonics are only due to ROM up to a maximum observed order of 22 and 27, for silica and PET respectively. With bulk plastic, which shows a maximum harmonic order due to CWE of 14, ROM harmonics up to the $22^{\text {nd }}$ order were generated. These observations are consistent with the theoretical descriptions of the two processes (see chapter 3.3).

In contrast to the plastic and silica targets, only harmonics via CWE could be generated with the carbon foils. Thanks to the ultra-high contrast of the laser pulses, we generated high-order harmonics from foils as thin as $7 \mathrm{~nm}$. We observed that the maximum harmonic order decreases slowly with decreasing foil thickness, with foils thicker than $35 \mathrm{~nm}$ having a maximum harmonic order of 18 , which is in agreement with the expected maximum plasma density of carbon. This is an indication that the initial plasma expansion is indeed small, as could be expected for laser pulses with an ultra-high contrast. However, at the highest-intensity focus (which we assume is the focus for optimum ion acceleration) the harmonic signal was found to be very weak. Among all investigated thicknesses of the carbon nanofoils, the strongest harmonic spectra were recorded (see figures 5.14f to 5.16f) when the target was placed out of focus by several hundreds of microns to a millimeter (either in front or behind the focus), where the intensity can be more than an order of magnitude lower. It appeared that thinner foils require a lower intensity (larger defocusing), however, no optimum focal position could be determined with the foils, because the number of shots per foil target were limited and the main attention was put on recording a sufficient number of ion spectra.

Before a further interpretation of the recorded data is presented, it should be noted that there was, unfortunately, a strong fluctuation in the strength of the harmonic signal of more than a factor of 2 (stronger than the fluctuation in the ionic spectra) with all targets. Harmonic spectra recorded from the carbon nanofoils possessed the strongest fluctuations as in many shots there were no harmonics observed at all. In addition, there was a fluctuation in the frequency of the harmonic peaks which shifted between consecutive shots. An example of 
this effect can be seen when comparing the spectra in figure 5.11(a) and (b), or in figure 5.14(a) to (c). It is uncertain what the cause of the fluctuations is and why in most cases no harmonic spectra due to ROM were recorded despite the expectation that the estimated drive laser intensity should be sufficiently high for HOHG by ROM. A possible explanation is that the focused intensity on target was lower than the estimated intensity $\left(3 \cdot 10^{19} \mathrm{~W} / \mathrm{cm}^{2}\right)$. However, based on the laser parameters and the profile of the focal spot as inspected with a microscope objective, this explanation seems unlikely. Another explanation that could account for the fluctuation in the data could be a shot-to-shot fluctuation in the laser pulse energy, or that the double plasma mirror was not functioning in a reproducible manner shot-to-shot, which could result in a strong fluctuation in the final pulse energy or the pulse contrast. Consequently, it could be that the conditions of the intensity or pulse contrast were optimum for HOHG via $\mathrm{ROM}$ in only a few shots.

Another explanation for the less frequently recorded ROM spectra can be found in the properties of the target material. In particular, the damage threshold of the target determines the moment of plasma formation and subsequent plasma expansion before the main pulse arrives, this is related to the peak intensity and the temporal profile of the pulse. For example, if the double plasma mirror did function properly and the contrast is assumed to be as high as the estimated value ( $10^{13}$ on the nanosecond time scale, see appendix A), it could be that, with the silica and plastic targets, the formed plasma gradient scale length is in fact too small for efficient HOHG via ROM. This is because silica and plastic are insulators that are transparent at the drive laser wavelengths up to a certain damage threshold intensity which is about $10^{12} \mathrm{~W} / \mathrm{cm}^{2}$ for sub-ps pulses [190]. This intensity level is reached when the contrast level is about $10^{-7}$, which occurs at about 200 fs before the peak of the pulse (see figure A.1 in appendix A). Taking a typical expansion velocity for the plasma (a few $10^{7} \mathrm{~cm} / \mathrm{s}$ [191], see also equation 3.3), in this case a typical plasma scale length would be formed that is smaller than 0.04 microns $(L / \lambda<0.05)$. This value is below the optimum scale length for $\mathrm{HOHG}$ via $\mathrm{ROM}(L / \lambda \approx 0.2$, see section 3.3.1). However, this interpretation does not account for the observed fluctuations. In order to ascertain the cause of both problems (fluctuations and the low number of cases where recorded harmonic spectra are due to ROM), a measurement of the temporal pulse profile and its shot-to-shot stability has to be performed. However, the most common method to measure the temporal pulse profile is to use a scanning third-order autocorrelator (such as the commercially available Sequoia third-order autocorrelator (from Amplitude Technology, see appendix A) which is also employed at PHI), which averages over several shots and is not able to determine shot-to-shot fluctuations. Such a measurement would require the installation of a different, single-shot thirdorder autocorrelator [192].

For the carbon foils, the situation is somewhat more complex. The harmonic spectra that were obtained with the carbon nanofoils posses somewhat more fluctuation in the intensity of the signal and the frequency of the har- 
monic orders (slightly shifted on the camera) as compared to the other target materials. In addition, the profile of the reflected IR beam coming from the carbon targets was not always centered. A possible explanation for this could be that the surface of the nanofoils was not perfectly flat in all cases, or was non-parallel with respect to the supporting frame due to left-overs of the ablation crater walls, although this was minimized. This could account for the somewhat stronger fluctuation in the signal strength.

A possible explanation why no harmonics due to ROM were recorded with the carbon foils is related to the transparency properties of carbon: compared to silica and plastic, which are insulators that are transparent at the drive laser wavelengths (up to a certain intensity), bulk carbon is absorptive in this range (already at low intensities) and thus has a damage threshold that is more than 3 orders of magnitude lower, as measured with nanosecond pulses [193]. For femtosecond pulses, the damage threshold of amorphous carbon is unknown, but could be expected to be lower than that of crystalline graphite which has a damage threshold that is more than 1 order of magnitude lower than that of silica $[190,194]$. In addition, since we use extremely thin foils, heat conduction is confined to two dimensions (instead of three in bulk targets) which further lowers the damage threshold of the carbon nanofoils for irradiation on the picosecond and nanosecond time scale. Based on these assumptions, it could be expected that, with carbon nanofoils, the formation of the preplasma starts at earlier times compared to plastic bulk targets. With the estimated pulse contrast of the UHI 100 laser of about $10^{13}$ on the nanosecond time scale, but with, e.g., $10^{10}$ at about 0.5 ps before the peak of the pulse (see figure A.1 in appendix A), one could expect plasma formation and expansion to start rather early, approximately 0.5 to 1 ps before the main pulse arrives [58]. Assuming again a typical expansion velocity for the plasma of a few $10^{7} \mathrm{~cm} / \mathrm{s}$ [191], in this case a larger plasma scale length of 0.1 to 0.2 microns (corresponding to $L / \lambda$ between 0.13 and 0.25 ) would be formed, i.e. the lower damage threshold reduces the steepness of the plasma gradient. When considering bulk targets, such a plasma density gradient is still sufficiently steep for efficient HOHG. However, as we will show in the next section via numerical modeling, this value might be beyond the optimum scale length for HOHG from extremely thin foils.

Furthermore, this interplay of the damage threshold with a time-dependent pulse contrast may have also been responsible for the observed changes in the harmonic spectra when the focal position was moved with respect to the target. When the foil targets were placed in the waist of the focus, the harmonics due to ROM would be too weak due to a too large plasma scale length, and, for harmonics due to CWE, the scale length could also then have been too large (beyond the optimum scale length) to facilitate transmission of the harmonic radiation through the plasma (see section 3.3.2). On the contrary, when the targets were placed out of focus, the intensity could have been too low to efficiently generate high-order harmonics via ROM, and only harmonics via CWE were observed because of the sufficiently short density gradient scale length. 
A verification of this interpretation would require a systematic investigation of the damage threshold for the carbon foils on the femtosecond time scale as a function of the foil thickness and a detailed characterization of the pulse contrast versus time.

In addition to the observation that the recorded spectra were stronger when the foils were positioned out of the laser focus, an intense spot profile was observed on the frosted glass behind the target in the propagation direction of the laser beam. The strength of this beam profile is strongly related to the strength of the harmonic signal from the front surface. For example, if the target was placed in the focus, this signal was hardly detectable. In order to obtain an indication of the wavelength of the radiation, a filter that blocks IR light was placed in part of the beam. From this, the signal strongly decreased in intensity, but some radiation still passed through the filter. It would be unlikely that this radiation is IR light that is transmitted through the foil after the foil has been expanded substantially because then this signal would be stronger when the target is placed in the focus position and the signal would decrease with increasing foil thickness. A possible interpretation is that this light consists of emitted harmonic radiation from the rear side of the foil. Such an effect was previously observed by other research laboratories where foils thicker than $50 \mathrm{~nm}$ were irradiated $[120,160,161]$. Also here, further investigation is required, e.g. with a spectrometer, to determine if this radiation is indeed harmonic radiation.

To summarize this subsection on HOHG, in spectra from plastic and silica targets high-order harmonics due to relativistic effects (ROM) were found, although this observation was not shot-to-shot reproducible so far. High-order harmonics have been generated also from extremely thin foils, as thin as $7 \mathrm{~nm}$, but the spectra have to be attributed to CWE. No relativistic harmonics due to ROM were observed with the nanofoils. Under the present experimental conditions, no enhancement of the harmonic efficiency was found, unlike that which was expected simply based on the higher intensity available from the drive laser (UHI 100 versus UHI 10). Also with the nanofoils, no enhancement was found with respect to bulk targets, as was suggested based on the similarity between ion acceleration and HOHG with respect to the absorption mechanisms and generation of hot electrons. When comparing the findings and assuming that the pulse has an ultra-high contrast, it seems that it is important to take into account the material properties, in particular the damage threshold, and the temporal pulse contrast of the pedestal in the picosecond time scale.

In order to investigate whether, under ideal conditions, the harmonic efficiency can be enhanced using foils with thicknesses in the transparent regime, we performed numerical calculations, which will be described at the end of the next section. 


\subsection{Numerical modeling}

In the field of laser-plasma interactions, numerical calculations prove to be crucial in understanding the processes that occur. Numerical modeling is used to interpret experimental results or predict new physical processes still to be verified experimentally. Here, we decided to perform particle-in-cell (PIC) calculations on the interaction with thin foil plasma slabs. We used the code from Paul Gibbon called Boosted Oblique-incidence Particle-in-cell Simulation $(\mathrm{BOPS})^{7}[62,195]$. It is a $1 \mathrm{D} 3 \mathrm{~V}$ (1 dimension, 3 velocities) PIC code that enables the numerical calculation of obliquely incident laser pulses onto a plasma profile.

PIC calculations are a valuable tool to investigate the collective behavior of the plasma. In PIC calculations, the motion of a large collection of charges (macro-particles) is numerically computed in their self-consistent electric and magnetic field in a continuous loop [114]. From the positions and velocities at a given time, the charge and current densities on a spatial grid is computed. From these charges and current densities, the electric and magnetic field is calculated via Maxwell's equations. Electromagnetic fields from the laser are also included. These fields are used to determine, via the Lorentz force, the new positions and velocities of the charges, from which the cycle continues.

Numerical modeling has been demonstrated to be highly useful for the interpretation of experimental results $[109,122,134,139]$. However several major difficulties remain. The first is a significant lack of knowledge concerning the initial conditions. This refers particularly to the width and shape of the plasma distribution in space and the average plasma density. Another difficulty is that the computation time increases when using higher densities and smaller spatial and temporal steps. To reduce such problems, many calculations so far have used only low to moderate densities (i.e., 10 to 100 times the critical density $\left.n_{c}[52,122,138]\right)$ which are much lower than what is expected from the full ionization of a solid. However, the plasma density is an important parameter to take into consideration as shown by d'Humieres et al. [122]. There it was observed in numerical modeling (using plasma densities less than $100 n_{c}$ ) that the laser absorption and maximum proton energy depend critically on the target density. In their calculations they assumed plasmas with initially a steplike profile which may deviate from experimental conditions. This assumption is generally made in numerical modeling because the initial plasma distribution is not known. However, this would correspond in experiments to a laser pulse without any pedestal or prepulse present, this is not a trivial assumption. An attempt to model the effect of a laser prepulse was done by Andreev et $a l$. [196]. Their numerical calculations, based on assuming a low plasma density (few times $n_{c}$ ) of micron size with a linear gradient, show altered maximum proton energies when the initial gradient was varied and indicate that there exists an optimal gradient for proton acceleration. However, since the target density influences the maximum proton energy, as observed by d'Humieres, it

\footnotetext{
${ }^{7}$ This code is available on the Internet
} 
remains unknown what the effect of a plasma gradient would be on the proton acceleration when using higher plasma densities.

Taking into account these two predictions on the plasma density and gradient, we decided to follow a different approach and model proton acceleration via a number of differently shaped density distributions, assuming high plasma densities. Two sets of numerical calculations were performed corresponding to the two sets of experiments we carried out with the UHI 10 and UHI 100 laser at PHI. The results of the first set of numerical calculations which correspond to the first experimental run with the UHI 10 laser on proton acceleration will be described in subsection 5.4.1, and can be found in our article [186]. We will explain the results of these numerical calculations which involve asymmetric linear plasma density gradients based on assumptions of an optimum foil thickness obtained in the performed experiments at PHI. Based on these numerically calculated results which are in qualitative agreement with the experimental results, we performed a second set of numerical calculations to study the influence of the pre-plasma gradient scale length on the maximum proton energy for different foil thicknesses. This will be discussed in subsection 5.4.2 [197]. In the calculations we used laser parameters that were similar to the experimental parameters of the second experimental run. From the second set of numerical calculations the harmonic signal was also analyzed. These results will be described in section 5.4.3.

\subsubsection{PIC calculations based on experiments with UHI10}

In experiments and numerical modeling, the energy of accelerated protons depends on the plasma density, the scale length and the laser parameters. In order to compare the numerical modeling with the experiments and interpret the experimental data, we used input parameters that corresponded closely to the experimental conditions. The calculations are based on an incident laser pulse which is p-polarized, having a sine-squared temporal shape with a duration of $60 \mathrm{fs}$ (FWHM), an intensity of $5 \cdot 10^{17} \mathrm{~W} / \mathrm{cm}^{2}$ and a wavelength of $800 \mathrm{~nm}$. The angle of incidence on the target was set to 45 degrees. As a test and as was expected [133], choosing s-polarization or normal incidence showed no acceleration.

Special emphasis was put on using a realistically high plasma density, i.e., which is equal to the rather high electron density obtained when fully ionizing solid-state carbon (344 times the critical density $n_{c}$ ). In the model this is addressed by scaling the number of particles with the thickness of the foil. The thickness of the foils varies from 5 to $200 \mathrm{~nm}$. Comparable with other reported numerical calculations (e.g. [52]), we used more than 200 electrons and ions per cell. The total number of cells was 150,000 with a cell size of $0.1 \mathrm{~nm}$. The calculations spanned a time of about $380 \mathrm{fs}$, which is sufficient for an efficient energy exchange between heated electrons and ions. The initial temperature of the electrons and ions was set to zero, following Dong et al. [52]. We verified that the accumulation of numerical errors (numerical heating) could be 
neglected during the considered time intervals, by running comparative numerical calculations, e.g. with zero laser intensity but otherwise equal parameters.

An essential point in our calculations is the implementation of a small initial gradient on the plasma density profile in order to try to include some plasma dynamics before the main pulse. This was done because numerical calculations, assuming initially step-like plasma profiles, could not reproduce the experimental results, as we will show below. Furthermore, the experiment with the UHI 10 laser (see section 5.3.2) indicated the existence of an optimum foil thickness, as described in section 3.2.2. For foils thinner than this optimum thickness, the formation of a shock wave induced by a pedestal or prepulse prior to the main pulse would cause the complete foil to expand. However, although it is believed that the expansion leads to an exponential gradient (see section 3.1.1) and approximate values for the expansion velocity are known (typically a few $10^{7} \mathrm{~cm} / \mathrm{s}$ [191]), the exact plasma profile and the scale lengths on the front and rear surface of the foil are not known. Hence, in the numerical calculations, we had to make certain assumptions and estimations.
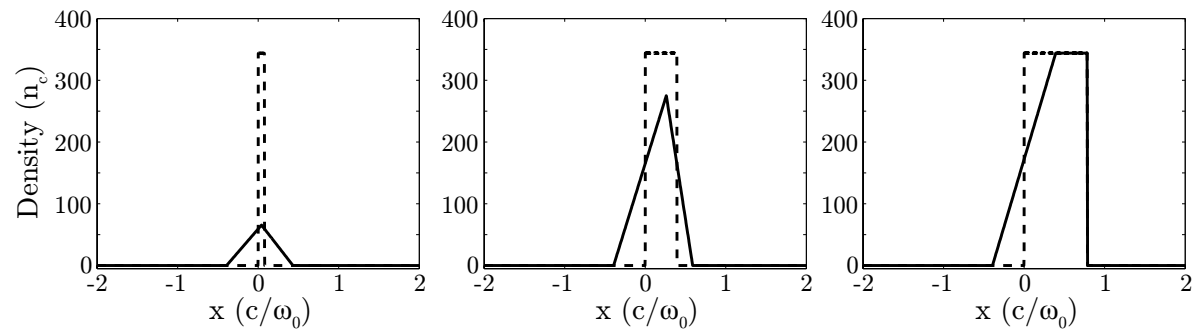

Figure 5.17: Three examples of initial plasma density distributions for foil thicknesses of $10 \mathrm{~nm}$ (left), $50 \mathrm{~nm}$ (middle) and $100 \mathrm{~nm}$ (right). In this frame, the laser pulse is incident from the left. The dashed traces correspond to profiles with an infinitely steep gradient. The solid traces correspond to the distributions obtained from our model, resulting after plasma heating and expansion from the front surface and, subsequently, from the rear surface.

Figure 5.17 shows three examples of plasma density distributions we used in the numerical calculations, based on the several assumptions and estimations in our model discussed below. The figure shows the initial density profiles for three different foil thicknesses; $10 \mathrm{~nm}$ (on the left), $50 \mathrm{~nm}$ (on the middle) and $100 \mathrm{~nm}$ (on the right). In this frame, the light is incident from the left. The dashed line, indicates the distribution of a step-like profile. The solid lines correspond to foils with a small initial scale length gradient.

In order to obtain an appropriate scale length that matches the experimental conditions we proceeded as follows. First, we assumed that the finite (and still substantial) intensity of the pedestal in front of the laser pulse fully ionizes the foil, heats electrons and induces a shock wave [198] with an average velocity, 
$v_{e}$, and that part of the electrons and the induced shock wave move through the foil with this velocity. Also, the heated electrons lead to an expansion of the plasma, at the front and rear surfaces of the foil, with the ionic sound speed $c_{s}$. Both velocities are actually unknown, however, we assumed that these values are the same for all of the considered foil thicknesses and the same on both sides of the foil. We believe this is to some extent justified because of the small thickness of the modeled foils and the associated low energy loss. We further supposed that the optimum foil thickness, $d_{\text {opt }}$, is provided by the thinnest foil for which the rear surface remains unperturbed when the main laser pulse arrives, as was described in section 3.2.2. This expresses the physical expectation that the shock wave will reach the rear surface of this optimum foil after a characteristic time delay $t_{p}$, such that $v_{e} t_{p}$ equals the optimum thickness $d_{\text {opt }}$. During the same time interval a gradient at the front has formed with a scale length of $L_{f}=c_{s} t_{p}$, as described by equation 3.3 in section 3.1. This situation is illustrated in figure 5.17, with the solid black line in the figure on the right, for the case of a $100 \mathrm{~nm}$ thick foil. The gradient at the rear surface for foils thicker than $d_{o p t}$ is infinitely steep.

Accordingly, for foils with a thickness, $d$, smaller than the optimum thickness (corresponding to the solid traces in the left and middle graphs of figure 5.17), the time for the electrons to reach the rear surface is $t^{\prime}=d / v_{e}$, which is less than the characteristic time delay. This means that during the time interval $t_{p}-t^{\prime}$ the rear side of the foil can also expand with the assumed expansion velocity $c_{s}$. We can estimate the gradient at the rear surface with the following equation, in which expressions for the ion and electron velocity have been substituted:

$$
L_{r}=c_{s}\left(t_{p}-t^{\prime}\right)=L_{f} \frac{d_{o p t}-d}{d_{o p t}} .
$$

As can be seen from this equation, the scale length at the rear surface depends on: the thickness of the foil, the optimum foil thickness and the scale length at the front surface which are unknown values. Thus in order to estimate the complete plasma density distribution to be used in numerical calculations, assuming values for the scale length at the front surface and the optimum thickness that match the experimental value, the associated scale length at the rear surface of any foil thickness $d$ can be calculated with this expression. In order to conserve the total charge for foils thinner than the optimum thickness, the maximum plasma density has to be scaled with the foil thickness.

Ideally, the plasma gradients at the front and rear surface have an exponential shape. In the PIC code we used, it was not possible to take different exponential gradients at the front and rear surface of the foil ${ }^{8}$. Instead, we took linear gradients, as used in calculations by Andreev [196], where the scale length (usually defined for exponential gradients) is equal to the width at half

\footnotetext{
${ }^{8} \mathrm{~A}$ new adjustment for the code has been written that allows calculations with density profiles having a different exponential gradient at the front surface and the rear surface. However, due to limited time, such numerical calculations have not yet been performed
} 
maximum to ensure that the number of particles in the expanded region is the same as with an exponential gradient. Three examples of the plasma density distribution obtained with the model are shown in figure 5.17. As can be seen from these figures, for initially very thin foils (figure on the left), the model yields a symmetric profile with a low peak value. And it yields various asymmetric profiles and a higher maximum density with thicker foils.

Only recently (after we performed our calculations), a similar approach was followed by Andreev et al. [199], who additionally used an estimation for the shock wave velocity. However, they again used a very low initial plasma density $\left(n_{0} \approx 6 n_{c}\right)$, this means that the laser interacts with a larger region of underdense plasma as compared to high density plasmas, for which the absorption and transparency can substantially differ, and consequently alter the maximum energy of accelerated ions.

In order to investigate the specific influence on proton acceleration of our choice of the initial plasma density profiles, in comparison to the rather unphysical and standard assumption of a rectangular profile, we performed comparing calculations using both types of initial plasma profiles. These calculations yielded spectra of the kinetic energy distribution of protons from which we extracted the maximum proton energy. This maximum proton energy is plotted in figure 5.18, on the left, as a function of the foil thickness. The right graph of the figure shows the calculated absorbed laser energy.
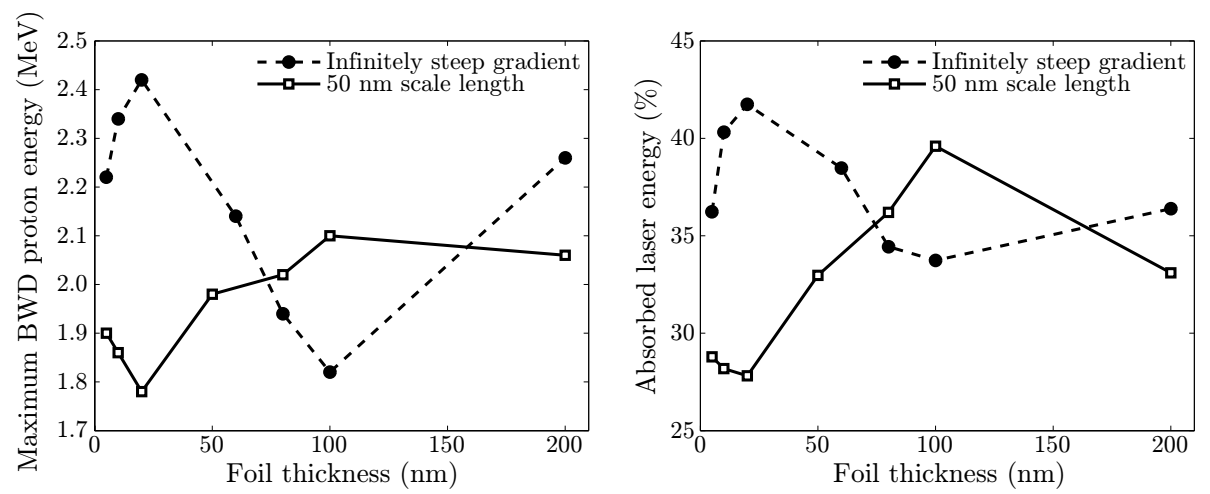

Figure 5.18: Calculated maximum proton energy in the backward direction (BWD, left) and absorbed laser energy (right) as a function of foil thickness. The filled circular symbols correspond to calculations with an infinitely steep initial profile and the open squared symbols are calculated with a $50 \mathrm{~nm}$ scale length at an optimum foil thickness of $100 \mathrm{~nm}$. The lines are guides for the eye.

It can be seen that, in the case of an infinitely steep initial plasma profile (zero scale length), the maximum proton energy shows a minimum for a $100 \mathrm{~nm}$ thick foil, and a maximum for a $20 \mathrm{~nm}$ thick foil. This trend is not the same as 
our experimentally observed trend in figure 5.9. To see how these predictions would alter when having a finite scale length, we performed numerical modeling with a finite plasma gradient. As can be seen in figure 5.18, where an optimum thickness of $100 \mathrm{~nm}$ (as was experimentally found by Ceccotti et al. [133]) and a scale length of $50 \mathrm{~nm}$ were chosen as input for equation 5.1, we obtained from the numerical calculations a maximum proton energy at the expected $100 \mathrm{~nm}$ thickness. It can also be seen that we obtained the expected decrease in proton energy with decreasing foil thickness. However, there were also deviations from that which is commonly expected (as based on rectangular profiles). Namely, with a finite scale length, i.e. when taking a pre-expansion of the plasma into account, as can be seen in figure 5.18, the proton energy decreases only to a minimum at around $20 \mathrm{~nm}$. Beyond this, i.e. with further reduced thickness we find a re-increase of the proton energy in the form of a maximum. When comparing this to our experimental data in figure 5.9, we find a good qualitative agreement.

What can also be seen when comparing the two graphs in figure 5.18, is that the maximum proton energy shows a similar trend to the laser absorption curve as a function of foil thickness; the minima and maxima are positioned at the same thicknesses.

A possible interpretation of the shown results can be as follows. For the thin foils (below $20 \mathrm{~nm}$ in the numerical modeling), the pre-expansion lowers the maximum plasma density substantially resulting in the main pulse interacting with a low density plasma. This means that a competition between two processes can occur. First, increasing the scale length decreases the accelerating electrostatic field formed by charge separation and therefore reduces the maximum attainable proton energies [134]. Second, increasing the scale length can increase the absorption of the main laser pulse [132], which results in a higher number of hot electrons and an increase in their temperature, thereby leading to a larger accelerating field. Apparently, with extremely thin foils, the increase in absorption is the dominant process and therefore results in a second optimum foil thickness in consequence of accompanying expansion.

To conclude, our experimental data agrees with our improved model that takes into account the limited expansion of the foils due to the finite pedestal of the ultra-high contrast drive pulse. It can also be seen in figure 5.9 that the numerical calculations predict that maximum proton energy peaks at extremely thin foils when the initial plasma density profile has an infinitely steep gradient. To investigate this prediction and obtain such a density profile, would require a higher pulse contrast in experiments.

\subsubsection{PIC calculations based on experiments with UHI100}

The data from the second experimental run, described in section 5.3.2, when supported with numerical modeling, should provide more information about the ion acceleration at extremely thin foils. In particular, our previously presented PIC calculations that correspond to the UHI 10 experiments suggest 
that, even with ultra-high contrast laser pulses, a plasma is formed with a density gradient which needs to be taken into account. To check this suggestion, also in the second set of numerical calculations, we studied the influence of the plasma density gradient on the maximum proton energy, and compared these results with the prediction of an enhanced proton acceleration in the transparent regime from calculations of others, as described in section 3.2.3.

At this point one has to recall that experiments with such thin foils and such high intensities had never been carried out before and, as we described above, there is currently no experimental means to determine the plasma density distribution at the time when the main pulse arrives. This means that the modeling of the results gained with the UHI 100 laser are rather explorative and only qualitative on the one hand. But on the other hand, they also present the first investigation of this kind in the transparent regime and thus provide original information from unexplored terrain. In the scope of this, it is worth mentioning an important difference from the calculations described before. These numerical calculations demanded long calculation times (up to 8 days for a single calculation), which limited the number of calculations that could be performed. Also, the calculations were already started before, and finished only after, the experiments with the UHI 100 laser. Hence, as opposed to the previous calculations, the optimum foil thickness for proton acceleration as obtained in the experiments was unknown at the time the numerical modeling was performed. This means that an approach similar to that used in the first set to estimate the density distribution (by calculating the scale length at the rear surface for certain assumed values for the scale length at the front surface) could not be followed.

Instead, in the calculations below with an initial scale length, we assumed a symmetrical exponential gradient on both sides of the foil because the foils of interest are very thin. The reason for this assumption is based on the results from the previous numerical calculations with regard to the UHI 10 experiments. From these calculations it could be seen that the maximum proton energy followed a trend similar to the trend for the laser absorption. Because the laser interacts at the front surface, the absorption is mainly determined by the gradient of the plasma at the front surface and, to a lesser extent, by the gradient at the rear surface. Furthermore, the density distribution of the extremely thin and expanded foils was found to be almost symmetric. In the same manner as in the above-mentioned numerical calculations, to conserve the total charge, we scaled the maximum plasma density.

In our numerical modeling, we used laser parameters that were predicted before the actual experiments at PHI were performed and which correspond closely to the experimental conditions; a p-polarized incident laser pulse, having a sine-squared temporal shape with a duration of 20 fs (FWHM), an intensity of $5.4 \cdot 10^{19} \mathrm{~W} / \mathrm{cm}^{2}\left(a_{0}=5\right)$ and a wavelength of $800 \mathrm{~nm}$. The angle of incidence on the target was set to 45 degrees. The total number of cells was 250,000 with a cell size of $0.05 \mathrm{~nm}$. A larger cell size of $0.2 \mathrm{~nm}$ was used for foils thicker than $200 \mathrm{~nm}$. An initial maximum plasma density of 110 times 
the critical density $n_{c}$ was taken, instead of $344 n_{c}$ that corresponds to fully ionized carbon, to reduce calculation time. In order to compare the numerical results with the experimental data, the foil thickness should be divided by the density ratio (344/110) to obtain the maximum proton energy as a function of the areal density, as in figure 5.10. In the numerical modeling, we restricted the calculation interval to $90 \mathrm{fs}$, which is sufficient for an efficient energy exchange between the heated electrons and ions and to determine the trend of the maximum proton energy. It should be noted that the final values for the maximum energies are somewhat higher when the calculation time is increased.

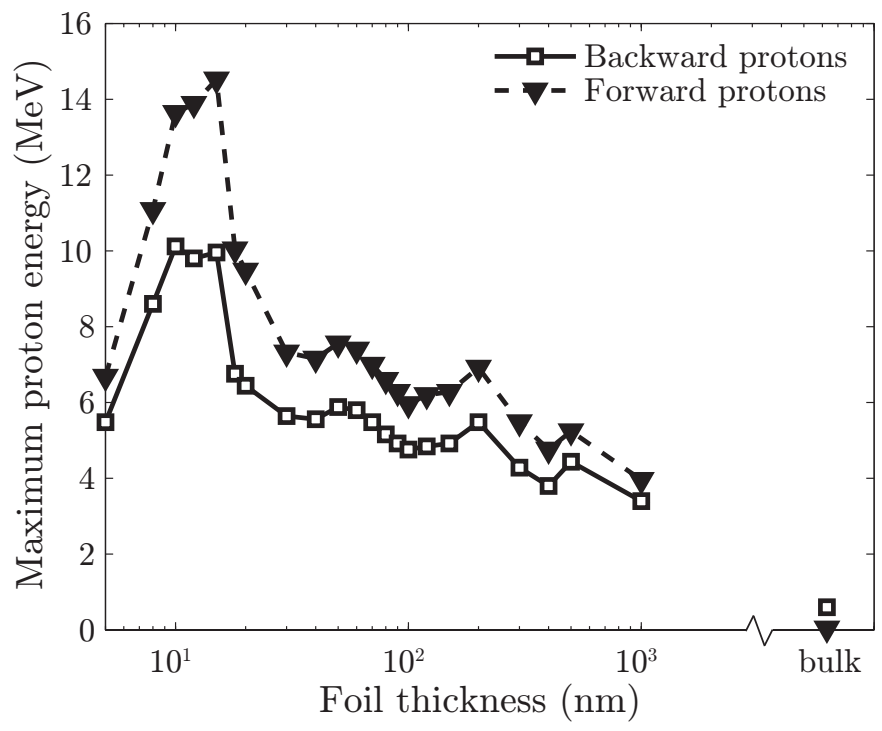

Figure 5.19: Calculated dependency of the maximum proton energy in the backward and forward direction on the foil thicknesses. The initial plasma density is assumed to have a standard, step-like profile $(L / \lambda=0)$, which neglects the pre-expansion of foils. Clearly visible is the enhanced peak for the thinnest foils (around 10 to $20 \mathrm{~nm}$ ), which corresponds to the transparent regime of TNSA. The opaque regime for foils thicker than $30 \mathrm{~nm}$ shows a slower decay of maximum proton energy. The low proton energy calculated for a bulk target is shown at the right of the graph.

In order to investigate the effect of a plasma scale length on the interaction of the laser pulse and the maximum proton energy and, because the exact scale length in experiments is unknown, we performed calculations with differently expanded plasmas and compared this with the predicted trend of the transparent regime. First, to confirm that an enhanced peak in the maximum proton energy is observed with our used laser parameters and, also to check 
if our calculations are consistent with the calculations by others [52,122], calculations were performed on several foil thicknesses using plasma profiles with an infinitely steep gradient. The motivation for such calculations is to allow a comparison with previous calculations of others, although this standard approach of an infinitely steep gradient, which is generally assumed in numerical calculations for ultra-high contrast laser pulses, could be somewhat unphysical. Secondly, different scale lengths (up to $L / \lambda=0.1$ ) were used in numerical modeling with foils having an initial symmetrically exponential density profile, as previously described. However, because of the time-consuming nature of the calculations, a fewer number of foil thicknesses and a limited number of scale lengths could be considered. The maximum energy in both the forward and backward direction was extracted from the kinetic energy distribution of the protons for several foil thicknesses. The standard results based on the assumption of an initially infinitely steep plasma gradient, thus neglecting the pre-expansion of the ionized foils, are shown in figure 5.19. As a comparison, the maximum value calculated for a bulk target is shown on the right side in the graph.

As can be seen from figure 5.19, the trend of the maximum proton energy is indeed very similar to the numerical predictions on ion acceleration in the transparent regime obtained by Dong [52] and d'Humieres [122], described in section 3.2.3. Our data also clearly shows the enhanced peak in the transparent regime, in this case for foils thinner than approximately $30 \mathrm{~nm}$. In accordance to the descriptions of Dong and d'Humieres, the maximum energy shows a strong decay below and beyond the optimum thickness and a weaker decay towards thicker foils in the opaque regime. As can further be seen in figure 5.19, in the forward direction, the peak value of the maximum energy of the accelerated protons in the transparent regime is approximately twice as high as the maximum energy at the transition to the opaque regime. In the backward direction the enhancement is somewhat lower. Actually, for all foil thicknesses, the maximum proton energy in the backward direction is somewhat smaller than in the forward direction. We attribute this to the small additional expansion during the laser pulse interaction at the front surface, consistent with the previously reported numerical calculations and experimental observations described in section 3.2 .

However, when comparing the curve in figure 5.19 with the experimental data in figure 5.10, we observe a dissimilar trend. As suggested by our previous experiments with the UHI 10 laser and the corresponding numerical modeling, we interpret the difference as a result of a pre-expansion of the plasma in the experimental conditions which is neglected in the standard numerical calculations assuming step-like density profiles. On the contrary, in the following set of calculations we took into account the pre-expansion of the plasma and investigated the influence of the plasma scale length on the maximum proton energy in the forward and backward direction, illustrated in figure 5.20 on the left and right, respectively, for the various foil thicknesses of interest. In this range of short scale lengths (up to $L / \lambda=0.1$ ), Brunel absorption and $\mathbf{v} \times \mathbf{B}$ heating 

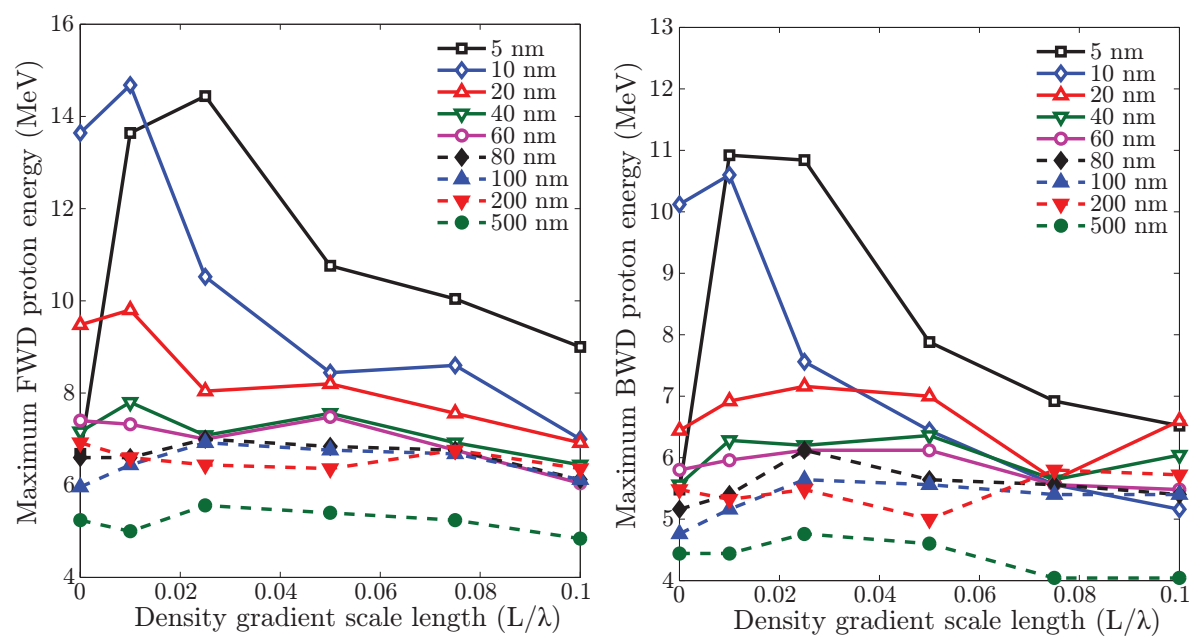

Figure 5.20: Calculated dependency of the maximum proton energy on the plasma density scale length for different foil thicknesses, in the forward direction (FWD) and in the backward direction (BWD). The lines are guides for the eye.

can be expected to be the dominating heating mechanisms, as described in section 3.1 .

What can be seen in figure 5.20 is that, in the case of the thinnest foils which correspond to the transparent regime, the proton energy is clearly the highest. However, there is a strong dependence on the scale length. Already beyond an optimum scale length of a very low value, there is a strong decrease in the maximum proton energy with increasing scale length. This means that extremely thin foils, in the transparent regime, are indeed the preferred choice for generating the highest proton energies, but this advantage can only be gained with a sufficiently high contrast of the laser. On the contrary, foils in the opaque regime are hardly sensitive to the short scale lengths considered in these numerical calculations. This robustness seems like a practical advantage in terms of more convenient laser specifications, however, it also means that the proton energy could have been much higher with the same drive laser pulse. Actually, the enhancement that can be achieved by moving into the transparent regime diminishes with increasing scale length, and the trend of the maximum proton energy versus the foil thickness flattens.

This flattening of the trend is illustrated in figure 5.21. For a better overview, in this figure we have replotted the data of figure 5.20, but now as a function of the foil thickness for the various scale lengths used in the calculations. It can be seen that the enhancement of using extremely thin foils is perished beyond an initial scale length of $L / \lambda \approx 0.05$. The flattened curve 

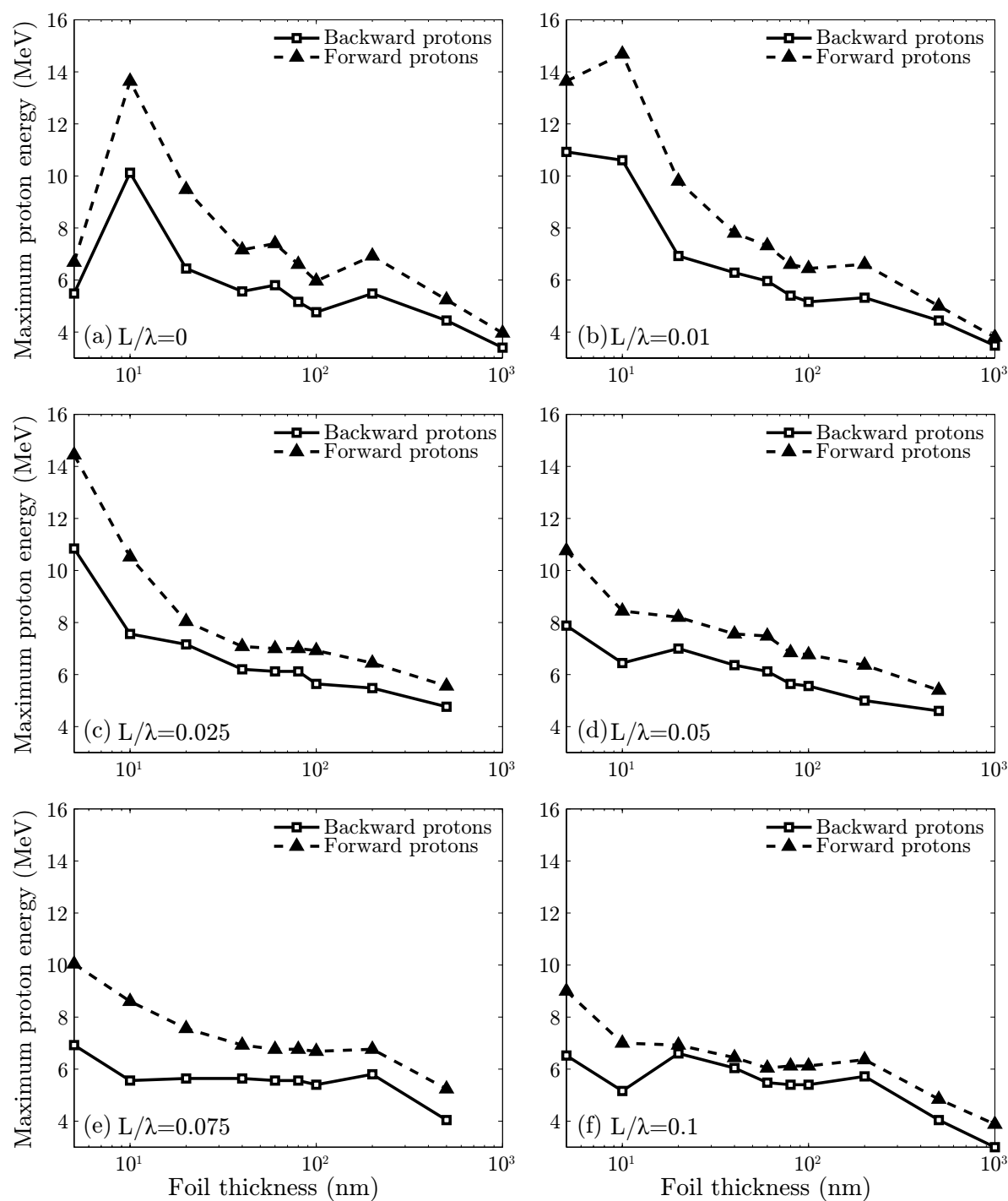

Figure 5.21: Calculated dependency of the maximum proton energy on foil thickness for different plasma scale lengths $L / \lambda$. Open squared symbols correspond to backward protons and filled triangular symbols correspond to forward directed protons.

for the maximum proton energy obtained with the relatively large scale length gradient $(L / \lambda=0.1)$ is qualitatively in agreement with our experimental data. This implies that our model, with the assumptions made on the density distribution, appears to be valid in the range of parameters used. This proves 
the correctness of the basic physical picture that in our experiments the main laser pulse interacts, not with an step-like plasma density profile, but with an expanded foil, the gradient of which is formed by the pedestal of the ultra-high contrast laser pulse. This conclusion means that even for such laser pulses with ultra-high contrast, it is important to take into account the intensity level of the pedestal on the picosecond time scale.

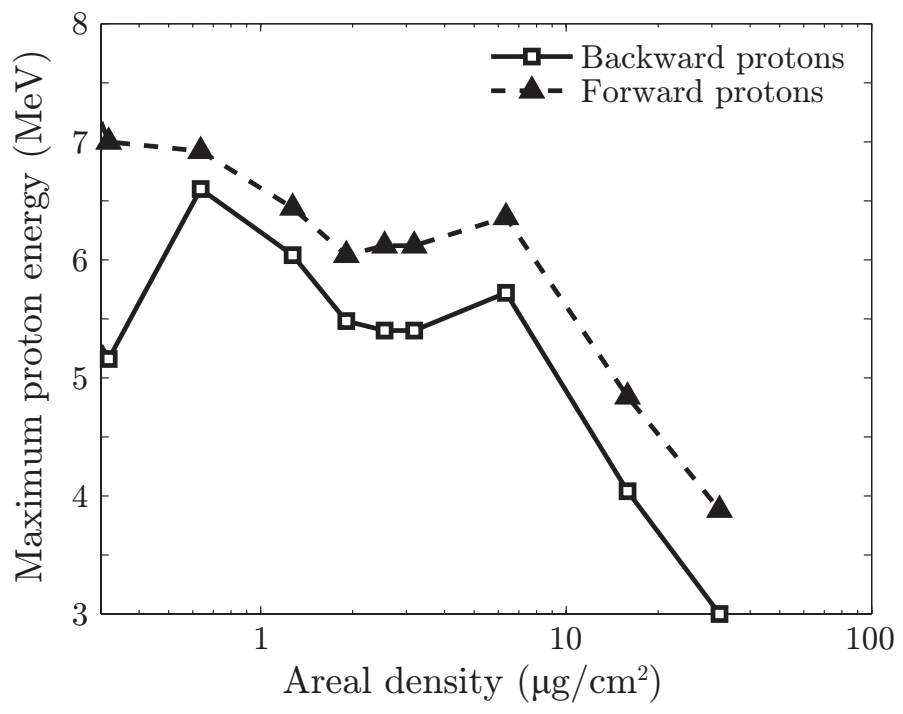

Figure 5.22: Calculated maximum proton energy as a function of the areal density (in the range of the experimental data in figure 5.10. In these numerical calculations, the initial plasma profile has a scale length of $L / \lambda=0.1$. Open squared symbols correspond to backward protons and filled triangular symbols correspond to forward directed protons.

Furthermore, when comparing the numerical results with the experimental data in more detail, we observe a strong similarity between both trends if an initial gradient scale length of approximately $L / \lambda=0.1$ is assumed in the numerical modeling. This similarity becomes more apparent if the maximum proton energy in figure 5.21f is plotted as a function of the areal density of the foils, as shown in figure 5.22, in the range of the experimental data (see figure 5.10). Note that the calculated data point in figure $5.21 \mathrm{f}$ corresponding to the $5 \mathrm{~nm}$ thick foil falls outside the experimental range and is not plotted in figure 5.22. In accordance with the experiment, in the backward direction two peaks are visible in the results from the numerical calculations as well; one peak in the opaque regime at thicker foils and one peak at foil thicknesses corresponding to the transparent regime. This implies that a scale length of $L / \lambda=0.1$ is approximately the gradient that appears to be present in our 
experiments. A somewhat improved similarity would probably be achieved with a slightly different initial scale length and with asymmetric density profiles as obtained using equation 5.1.

To conclude, our improved model that takes into account the limited expansion of the foils caused by a finite pulse contrast agrees well with our experimental data obtained with ultra-high contrast, high-intensity laser pulses. We can conclude that extremely thin foils, in the transparent regime, lead to the highest maximum proton energies. To reach this regime, it seems qualitatively known that a high pulse contrast is of importance when specifying the properties of a laser system. However, our comparative analysis between experimental data and numerical modeling, is the first quantification of such kind. It has been shown that the enhancement of the maximum proton energy with foils in the transparent regime requires a rather small scale length, i.e. an almost step-like plasma profile in numerical calculations, and consequently an extremely-high pulse contrast in experiments. With an increasing plasma density scale length, the advantage of ion acceleration in the transparent regime over the opaque regime decreases, resulting in a flattening of the trend for moderate plasma scale lengths $(L / \lambda \approx 0.1)$. To make use of the important potential of the transparent regime, our investigations prove clearly that to reduce the pre-expansion, further progress has to be made with respect to the laser pulse parameters, or to the target, by developing, e.g., freestanding nanofoils with a higher damage threshold than carbon.

\subsubsection{PIC calculations on high-order harmonic generation}

We suggested in chapter 3.4 that also HOHG may be enhanced in efficiency when utilizing extremely thin foils, in a similar manner as the predicted enhancement for ion acceleration because much of the underlying physics for both processes are the same, in particular the heating of electron by the Brunel effect. However, to qualify this statement, since the experimental results on HOHG showed no significant harmonics due to ROM, we further investigated this suggestion via numerical modeling.

Interestingly, the numerical calculations on the ion acceleration in the transparent regime using infinitely steep plasma density profiles and short laser pulses $\left(a_{0}=5\right.$ and $\tau_{\text {FWHM }}=20$ fs $)$ show a similar trend for HOHG via ROM as well. This is illustrated in figure 5.23, which shows, as the black squared symbols (black lines), the calculated dependence of the harmonic efficiency on the foil thickness for the $20^{\text {th }}, 40^{\text {th }}$ and $60^{\text {th }}$ high-order harmonics. This figure also shows the efficiency when assuming an exponential gradient profile with a scale length of $L / \lambda=0.1$ (grey triangular symbols, grey lines). As can be seen in the graphs, when the laser pulse interacts with targets which have a perfect step-like plasma density profile $(L / \lambda=0)$, the efficiency is the same for the bulk target (plotted on the right in the graphs) and the foils in the opaque regime (thicker than $30 \mathrm{~nm}$ ), while in the transparent regime the harmonic efficiency shows a peak. It can be seen that the maximum efficiency in the transparent 
regime is approximately a factor of 5 higher than the efficiency obtained with a bulk target. It is worth mentioning that in this transparent regime, the light wave in the forward direction (behind the plasma foil) also contains high-order harmonics with an efficiency that is comparable to the efficiency of the reflected harmonic spectra of the thicker foils.
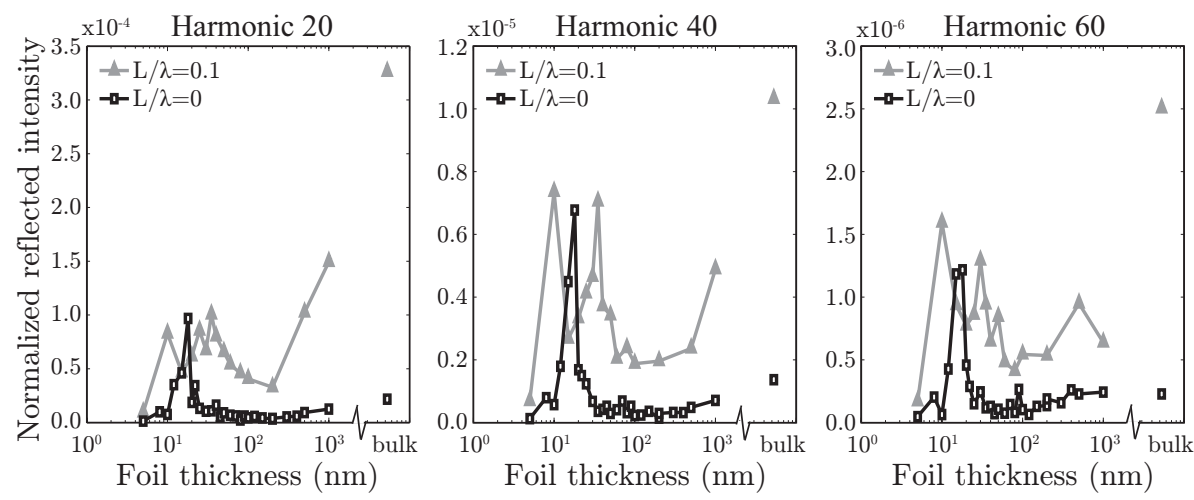

Figure 5.23: Calculated efficiency of the, respectively, $20^{\text {th }}, 40^{\text {th }}$ and $60^{\text {th }}$ harmonic order, for the interaction of a high-intensity laser pulse with a step-like plasma density profile (black squared symbols) and an expanded plasma with a gradient scale length of $L / \lambda=0.1$ (grey triangular symbols). The calculated efficiency for a bulk target is shown at the right of the graphs.

On the contrary, our calculations show that if the laser pulse interacts with targets that have a small initial gradient with a scale length of $L / \lambda=0.1$ (triangular symbols, grey lines), the efficiency is enhanced for all target thicknesses, expect for the foils that constitute the peak in efficiency with a step-like plasma profile. However, if there is a small initial gradient, the highest harmonic efficiency is predicted with the bulk target. Note that this value for the efficiency is even higher than the maximum value obtained with extremely thin foils having step-like profiles which would make it ineffective to use thin foils for backward HOHG via ROM. In an additional calculation, assuming a bulk target with an initial gradient with a scale length of $L / \lambda=0.2$, the harmonic efficiency is even higher as compared to a bulk target with a scale length of $L / \lambda=0.1$. This enhancement of the harmonic efficiency for bulk targets with a small gradient compared to a step-like profile, is consistent with the previously observed results in calculations and experiments that there is an optimum scale length for HOHG, as was discussed in chapter 3.

When comparing the findings from the numerical calculations with our experimental data, described in section 5.3.3, the interpretation of the laser damage threshold of the nanofoils and the related pulse contrast seems to be consistent. From the calculations, a small gradient of $L / \lambda=0.1$ results in 
an increased efficiency with the bulk target of an order of magnitude higher than without a gradient, and the optimum scale length is expected at somewhat larger values. However, as discussed in section 5.3.3, when the plasma mirrors did function properly and the contrast is assumed to be as high as the estimated value of $10^{13}$ on the nanosecond scale, we deduced that the gradient scale length could be smaller than 0.05, which is much smaller than the optimum scale length. The harmonic output corresponding to such a gradient $(L / \lambda<0.05)$ could then be too weak to be detected. In contrary to the bulk target, a scale length $L / \lambda$ present at the nanofoils, which was estimated to be between 0.13 and 0.25 in our experiments (see section 5.3.3), is not necessarily beneficial for HOHG. Furthermore, the enhancement factor for foils in the opaque regime, which is the range of most of the foil targets used for HOHG in our experiment, is much smaller than for the bulk target. This would also explain why, in our experiments, no harmonics due to ROM were detected from the nanofoils.

In summary, in the theoretically ideal situation that the laser pulse interacts with, initially, step-like plasma density profiles which, experimentally, would require either laser pulses with an extremely-high contrast or targets with a very high damage threshold or both, calculations show an enhancement of the efficiency for higher order harmonics with foil thicknesses in the transparent regime. Such a peak is also present in the calculated trend of the maximum proton energy versus the foil thickness. However, when the laser pulse interacts with an pre-expanded foil plasma, the enhancement in the maximum proton energy and the harmonic efficiency with foils in the transparent regime diminishes. Actually, the highest efficiency of HOHG can be achieved when utilizing a bulk target with a small (optimum) gradient scale length.

A possible explanation as to why a small density gradient is detrimental for ion acceleration and HOHG in the transparent regime, but beneficial for HOHG with bulk targets, can be found by examining the coefficients for the absorption, reflection and, transmission of the laser pulse and the evolution of the plasma electron density during the laser pulse. This will be explained in the following paragraphs. Figure 5.24 shows the coefficients, which are extracted from the numerical calculations described above, as a function of the foil thickness, when assuming an initially, step-like plasma density profile (a), and assuming a small initial gradient with a scale length of $L / \lambda=0.1$ (b). Examples of the evolution of the electron density in time during the interaction of an ultrashort high-intensity laser pulse are visualized in appendix F. In these calculations, a shorter pulse of $10 \mathrm{fs}$ FWHM had to be assumed to reduce the calculation time. The laser pulse is incident from the left. The cases compared in appendix $\mathrm{F}$ are a bulk target and a foil in the transparent regime, both with and without a density gradient. These figures illustrate the high complexity found in the interaction of ultra-intense lasers with the plasma, and that the initial conditions, due to the high nonlinearity of the interaction are of major importance. The figures F.1 to F.4 in appendix F are also shown in reduced 

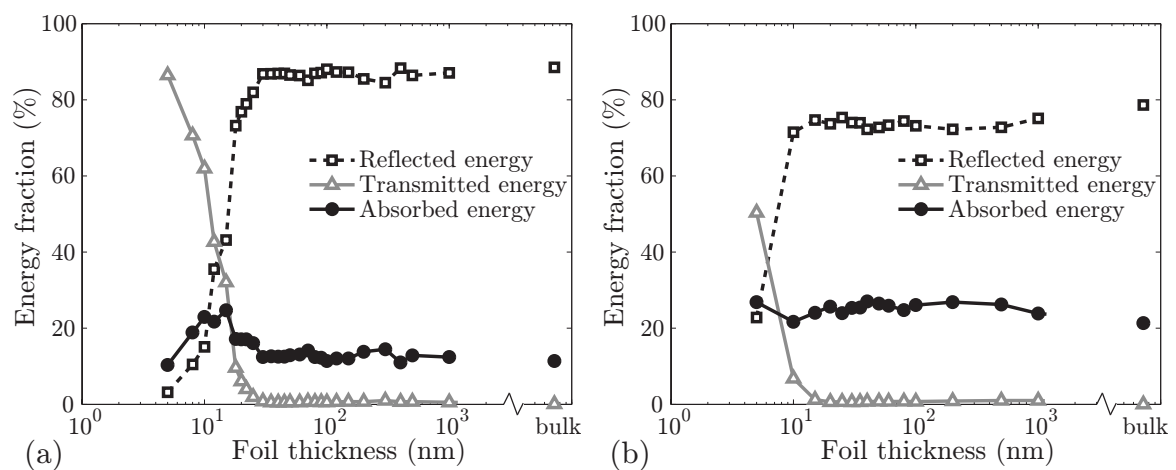

Figure 5.24: Calculated fractions of laser pulse absorption, reflection and transmission with assuming a step-like initial plasma density profile (a), and a small initial gradient with a scale length of $L / \lambda=0.1$ (b). The calculated values for a bulk target are shown at the right of the graphs.

size in figure 5.25. Note that the horizontal axes of the two plots illustrating the interaction with a bulk target have a different scale than the plots illustrating the evolution of the nanofoils.

What can be seen by comparing the evolution of the electron density of a foil in the transparent regime (figure F.3 and figure 5.25c) with that of a bulk target (figure F.1 and figure 5.25a) when both initially have a step-like density profile, is that the electron excursion is, indeed, larger with the foil target. The stronger excursion can account for the higher harmonic efficiency obtained with the foil. However, when comparing these density evolutions with that of the foil and the bulk target when both initially have a small gradient of $L / \lambda=0.1$ (figures F.4 plus 5.25d and figures F.2 plus 5.25b), the electron excursion is the strongest and most regular and periodic with the bulk target having the small gradient. This target results in the strongest harmonic output. With the bulk target the Brunel electrons that are pushed back from the vacuum on the left side into the target are lost in the bulk plasma and induce plasma oscillations when the target has a small initial gradient. Note that these plasma oscillations are a signature for the emission of high-order harmonics via CWE (see section 3.3.2). In contrast, with the foil targets, many of these Brunel electrons that are pushed back from the vacuum pass through the foil and return and intermix in a complex and irregular fashion with the remainder of the electrons in the foil, this could affect the process of HOHG and could be a reason why the efficiency of $\mathrm{HOHG}$ in this case is less than predicted from the interaction with an expanded bulk plasma.

The stronger electron excursion observed in the figures that visualize the evolution of the electron density can be related, qualitatively via energy con- 

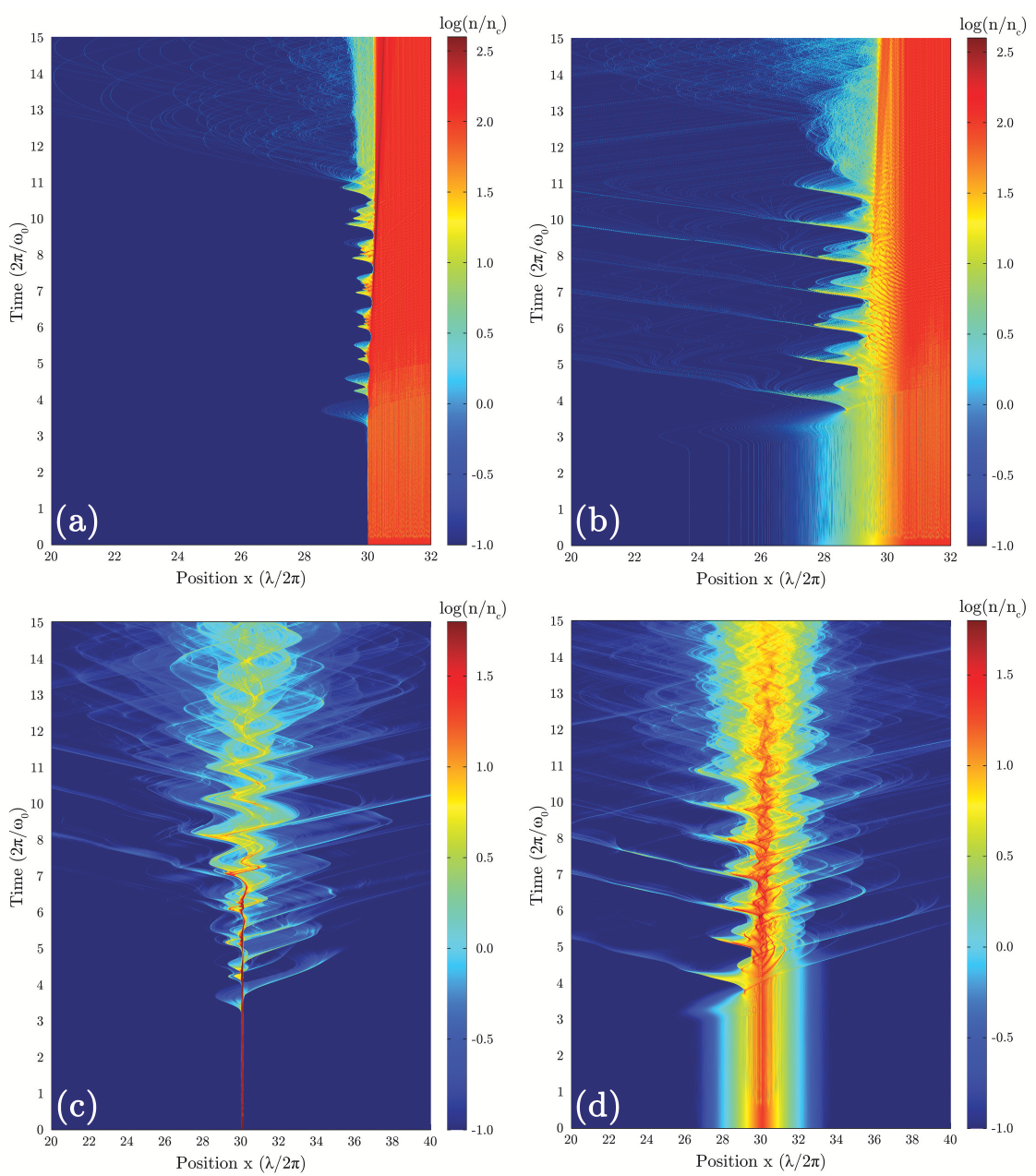

Figure 5.25: The evolution of the electron density in a logarithmic color scale in space and time for four different foil targets. The sub figures (a) to (d) are the images of appendix F reduced in size. The targets are (a) a bulk with a step-like initial density profile, (b) a bulk with initially a gradient profile with a scale length of $L / \lambda=0.1$, (c) a nanofoil (thickness of $0.1 \lambda / 2 \pi$ ) with a step-like initial profile, and (d) a nanofoil (thickness of $0.1 \lambda / 2 \pi$ ) with initially a gradient profile with a scale length of $L / \lambda=0.1$.

servation, to the absorption fraction. It can be seen from figure 5.24, that when the laser interacts with a step-like density profile (figure 5.24a), there is a peak in the absorption at foil thicknesses in the transparent regime, this is very 
similar to the peak observed for the maximum proton energy and the harmonic efficiency. The maximum absorption in the transparent regime is a factor of 2 higher than the absorption in the opaque regime. Compared to this trend, the absorption curve obtained with expanded foil plasmas (figure 5.24b) lies higher for all foils but is almost flat as a function of the foil thickness. This flattening of the curve is similar to the observed trend for the maximum proton energy.

The observation that the absorption follows a similar trend as the maximum proton energy is consistent with the same observation in the numerical calculations with regard to the experiments performed with the UHI 10 laser (see section 5.4.1). The numerical modeling with parameters that correspond with the experiments with the UHI 100 laser, further supports our interpretation (discussed in section 5.4.1) that the maximum proton energy depends on the competition between the absorption and the scale length. First, increasing the scale length lowers the plasma density which can increase the absorption of the main laser pulse. Consequently, this results in a higher number and temperature of hot electrons which results in an enlargement of the accelerating field responsible for proton and ion acceleration and an enhanced efficiency of HOHG from most targets. Second, increasing the scale length decreases the accelerating electrostatic field formed by charge separation (see equation 3.7) and accordingly reduces the maximum attainable proton energies.

\subsection{Discussion}

In this chapter, we presented the results of experiments on ion acceleration and high-order harmonic generation (HOHG) from solid targets with the UHI 10 and UHI 100 laser at PHI. We showed that high-order harmonic generation via CWE leads to the emission of coherent harmonic radiation. We also confirmed in more detail the intensity-dependent phase, as predicted by the current model of CWE and showed that, with this model, the dynamics of the plasma electrons can be derived on the attosecond time scale from the measured intensity profile due to the interference of multiple phase-synchronized sources of HOHG.

We also performed experiments on ion acceleration by target normal sheath acceleration with extremely thin foils (down to $1 \mathrm{~nm}$ thickness), i.e. in the range of the transparent regime, in order to test the validity of the theoretical predicted enhancement in this regime and, to identify the actual requirements on the laser and the target for optimum output. However, the use of such extremely thin foils in a freestanding manner is not trivial. The procedure to prepare flat carbon nanofoils has been described in detail in this chapter.

The main conclusion that can be drawn from our experiments with the UHI 10 and UHI 100 laser is that the theoretical predicted trend of an enhanced maximum proton energy in the transparent regime requires further development of the laser pulse contrast and the target. Although we irradiated these nanofoils with ultra-high contrast laser pulses, the experimental results do not agree with the predicted trend based on the assumption of initially infinitely steep plasma profiles. From our improved modeling in numerical 
calculations that qualitatively agree well with our experimental data, we can conclude that the limited expansion due to the finite pedestal of the ultra-high contrast drive laser pulse has to be taken into account. Furthermore, we found that the influence of an increasing plasma density scale length on the maximum proton energy is more detrimental for foil thicknesses in the transparent regime than for foils in the opaque regime. The maximum proton energy we obtained in both the transparent regime and the opaque regime was around $3 \mathrm{MeV}$ with the UHI 10 laser and around $6 \mathrm{MeV}$ with the UHI 100 laser. By comparing the experimental results with the numerical calculations, we estimated that under the present conditions, a scale length, $L / \lambda$, in the range of about 0.1 to 0.2 would be formed, which is supported by the experimental results on HOHG from the extremely thin carbon foils.

We generated high-order harmonics from foils as thin as $7 \mathrm{~nm}$. However, the pre-plasma formation due to the finite contrast appeared to be too large for efficient harmonic generation. Indeed, when the targets were placed out of focus, the harmonic signal became stronger due to the smaller expansion at a lower intensity. However, due to the lower intensity, only harmonics due to CWE and no relativistic ROM harmonics could be generated in that condition. The estimated scale length $L / \lambda$ of about 0.1 to 0.2 formed at these foils is around the optimum value for efficient HOHG with bulk targets, however, from numerical calculations it was found that such a gradient does not result in a much larger efficiency with foil targets, in contrast with bulk targets. However, not much harmonics due to ROM were recorded with bulk targets as well. This was due to the higher damage threshold of these targets, possibly the formed plasma gradient had a scale length that is too small for efficient $\mathrm{HOHG}$ by ROM.

The numerical calculations on $\mathrm{HOHG}$ in the relativistic regime show that in the transparent regime with step-like plasma profiles, the efficiency of harmonic production is predicted to increase in comparison to bulk targets with a step-like initial density profile. This is due to a stronger and denser electron excursion into the vacuum. However, it can be seen from the calculations that the highest efficiency is obtained, not with such foil targets, but with bulk targets having initially a small plasma density gradient. The trend for HOHG with step-like plasma profiles is similar to the trend for the maximum proton energy as a function of the foil thickness. The calculations show that the trends for the maximum proton energy and the harmonic efficiency versus the foil thickness exhibit a strong correlation with the absorption coefficient of the laser pulse.

Achieving ideal conditions in experiments (step-like plasma density profile) is expected to result in an enhanced maximum ion energy. The main conclusion with regard to the experimental requirements is that, in the transparent regime with extremely thin foils, it seems to be important to consider the pulse contrast in the picosecond time scale and the damage threshold of the material. The results of our experiments with ultra-high contrast laser pulses and the numerical calculations also suggest that both HOHG and ion acceleration in the trans- 
parent regime, as predicted for step-like plasma profiles, is experimentally very difficult to achieve. This is because, for demonstrating the theoretical predictions and to making use of the important potential in the transparent regime, an even higher pulse contrast would be required than currently is available with state-of-the-art technology. Instead, it seems more promising to realize improved targets that are as thin as our present targets (nanometer scale) but which posses a much higher damage threshold (preferably a dielectric with a low density, or, e.g., diamond-like carbon (DLC) nanofoils). A central issue would then be to fabricate such nanofoils and provide them as freestanding.

Another point of improvement, as concluded from our experimental work, is the shot-to-shot stability of the output of HOHG and proton acceleration. In order to quantify the cause of the fluctuations in the harmonic and ionic spectra it would be necessary to perform single-shot third-order autocorrelation measurements to determine exactly the peak power and the temporal pulse profile including the pedestal, prepulses and ASE, and to determine the shotto-shot reproducibility of both.

The limitations shown in the experimental conditions can have further implications for other experiments on high-intensity laser-matter interactions. The difficulty of improving the laser pulse's temporal profile to an even higher contrast with respect to the pedestal makes it questionable if it will ever be possible to ensure that the laser pulse interacts with a plasma profile having an infinitely steep gradient, as is presumed in many of the previous numerical calculations. For example, other regimes, such as the so-called radiation pressure acceleration (RPA) $[124,200]$ assume in their numerical modeling, a step-like plasma profile and then predict that nanometer-sized foils would result in an enhanced energy of the ions when compared to TNSA. Our experimental and numerical investigation show, for the first time in the TNSA regime, that even the development of smallest (finite) scale lengths can be detrimental for ion acceleration. It would thus be worthwhile to investigate the influence of finite scale lengths in other regimes as well. 
A scientist's aim in a discussion with his colleagues is not to persuade, but to clarify.

Leó Szilárd (1898 - 1964)

\section{6 \\ Conclusions and discussion}

In this thesis we presented information about the research on high harmonic generation and ion acceleration with high-intensity lasers to produce beams of coherent XUV and energetic ions of ultrashort duration, low-divergence and high-brightness. Possible applications for such beams can be found, for example, in medicine and fundamental research, such as isotope production for PET scanners, radiography, or probing electron dynamics on the attosecond time scale.

Ion acceleration can occur when a solid target is irradiated with a highintensity laser pulse. High harmonics can be generated from the interaction with such a pulse with either a gaseous medium or a solid-state medium. Each process has its own requirements for particular properties of the target and the used laser pulses; for example, the pulse intensity, the duration and the temporal contrast. For this reason, the experimental research described in this thesis has been performed with two different laser systems: the $12 \mathrm{TW}$ laser at the Laser Physics and Nonlinear Optics group (LPNO) and the $10 \mathrm{TW}$ and 100 TW laser at the Physique à Haute Intensité group (PHI).

To take full advantage of the current and future applications of ion acceleration and harmonic generation, both these processes require an enhancement of the conversion efficiency of the flux and maximum energy of the accelerated ions and the harmonic radiation with respect to the incident laser energy. This thesis aimed to investigate methods towards achieving this goal via an improved understanding of the underlying physics to identify the actual requirements of the laser and the target for optimum output.

In view of this, part of the work described in this thesis concerned the design, construction and testing of a setup and the required diagnostics for high har- 
monic generation in gaseous media (HHG). This was done with a view to make use of the TW laser at LPNO for a future exploration of several novel methods to enhance the efficiency of HHG. We constructed a setup that used a capillary waveguide for the generation of high harmonics in gases because such a configuration gives several advantages. The first is a higher output compared to that of gas jets because of an extended interaction length. The second is that the temporal and spatial coherence of the harmonic beam is improved by the selective build-up of high harmonics due to the short electron trajectories only. Furthermore, a capillary waveguide offers additional enhancement methods, for example, quasi-phase-matching via multi-mode beating.

Within the capillary section itself we implemented a novel approach of differential pumping. Outlet slits in the capillary were used to minimize reabsorption of the high harmonics behind the interaction region. With an XUVCCD camera and a movable transmission grating we were able to record both the harmonic spectrum and the beam profile. We obtained single-shot spectra, illustrating a relative high efficiency of HHG and detection in our setup. Firstly, harmonic spectra from Xe and Ar were recorded and the results were consistent with previously reported data, indicating that the apparatus was working properly. Secondly, we demonstrated an enhancement of HHG via harmonic excitation (HEx) in a guided-wave geometry for the first time. This was a step into a novel direction where a further enhancement of the harmonic output via HEx in ions from a capillary discharge in combination with quasiphase-matching, is aimed for.

As the alternative to high harmonic generation in gaseous media, this thesis also described the investigations of the generation of high-order harmonics from solid targets with the laser at PHI. This laser delivers laser pulses with an ultra-high contrast, which is required to minimize the plasma expansion caused by the ASE, pre-pulses and the pedestal of the pulse.

By recording interference patterns from three phase-synchronized sources of high-order harmonic generation (HOHG), we have shown, for the first time, that HOHG via coherent wake emission (CWE) leads to the emission of coherent harmonic radiation. Using these interference patterns, we have also shown that the dynamics of the plasma electrons during the interaction with the high-intensity laser pulse can be derived on the attosecond time scale.

The special properties of the laser at PHI also make this laser particularly suitable for the acceleration of ions from solid state targets. This was also investigated in this thesis. Special attention was given to the preparation of freestanding nanofoils and the interaction of such extremely thin foils with highintensity laser pulses. This experimentally unexplored regime of laser-matter interaction had only been theoretically investigated by other groups previously and promising predictions had been made. In particular, it had been predicted that the maximum ion energy would increase in the transparent regime due to increase in the temperature and density of hot electrons. However, this prediction was based on the standard assumption of a plasma density distribution 
starting with an infinitely steep gradient. Using nanofoils with thicknesses as thin as $1 \mathrm{~nm}$, i.e. in the order of the skin depth of the laser light, allowed us to experimentally investigate this regime for the first time. Thus, we could better evaluate the limits of the standard model and identify the actual requirements for the laser and the target for optimum output.

The main conclusion that can be drawn from our experiments with extremely thin foils is that, even when utilizing ultra-high contrast laser pulses, the target develops a significant pre-expansion which changes the shape of the plasma density distribution and thereby largely determines the interaction with the main pulse. This affects the maximum proton energy in ion acceleration and, in $\mathrm{HOHG}$, it influences the maximum harmonic frequency that can be achieved.

Another conclusion of our work is that for the case of ion acceleration, the experimental trend we observed (with the $10 \mathrm{TW}$ as well as with the $100 \mathrm{TW}$ laser) with regard to the maximum proton energy versus the foil thickness, is clearly dissimilar from what had been theoretically predicted previously. Instead of a single, high peak of the maximum proton energy in the range of nanometer thick foils, we observed a certain variation of the maximum energy as a function of the foil thickness. This was around a maximum proton energy of $3 \mathrm{MeV}$ with the $10 \mathrm{TW}$ laser and around $6 \mathrm{MeV}$ with the $100 \mathrm{TW}$ laser. This variation comprises a peak in the proton energy at around hundred nanometer foil thickness as was expected, which is far beyond the transparent regime. Further reducing the foil thickness resulted in the expected decrease of the maximum proton energy and can be ascribed to the expansion of the entire foil, as was previously explained by others. However, for extremely thin foils, in the transparent regime, we observed an unexpected second increase of the maximum proton energy, with the peak energy being about the same as with much thicker foils.

To better understand the physical reasons behind these findings, we carried out some numerical modeling with a particle-in-cell code. We found good agreement between the model and the described trend of the measured maximum proton energy. However, the agreement was achieved when a significant pre-expansion of the foil plasma towards a finite scale length was taking into account. This was associated with a reduced gradient of the plasma density. We found that the experimentally observed trends could not be explained with the standard presumption of the sharp, step-like plasma profile (zero scale length with infinite gradient) which had so far formed the basis of previous theoretical descriptions in the transparent regime. The finite initial gradient would be caused by the remainder of the pedestal of the laser pulses used, although these pulses were optimized to a record value of ultra-high pulse contrast. We showed that the effect of an increasing scale length is of increasing importance when reducing the foil thickness to reach the transparent regime. This means that reaching the benefits of an enhanced maximum proton energy with foils in the transparent regime requires a rather small scale length, i.e., an almost step-like plasma profile is to be provided. Improving laser-based ion accelera- 
tion thus requires, not only increased peak powers of the drive laser, but also that the pulse contrast of the drive laser and its specific effect on the selected foil is considered to be an absolutely central point. Otherwise, with an increasing plasma density scale length, the advantage of ion acceleration in the transparent regime over the opaque regime decreases, and one merely obtains a flattening of the trend for moderate plasma scale lengths $(L / \lambda \approx 0.1)$.

To make use of the important potential of ion acceleration in the transparent regime, our investigations clearly prove that further efforts have to be made to reduce the pre-expansion of the foils, even when the pulse contrast of the drive laser cannot be easily increased anymore. As an approach to this situation we suggest that novel types of freestanding nanofoils should be fabricated from other materials, in order to significantly increase the damage threshold. In other words, such materials would have to be highly transparent in the infrared range of the drive laser wavelength to increase the ionization threshold. As an example, oxide materials can be grown layer-by-layer at atomic precision with pulsed laser deposition [201]. Compared to carbon, such oxides might yield increased damage thresholds by some orders of magnitude due to their large band gap energies and transmittivity to infrared light.

With such an approach it can be expected that HOHG would also improve via the use of extremely thin foils in the transparent regime. We can conclude this from our numerical modeling of HOHG via the process of the relativistic oscillating mirror, which we have performed for the first time in the transparent regime. The results show that, when a step-like plasma profile can be realized, the harmonic efficiency should increase to values beyond that obtainable from bulk targets. The calculations also predict that a small initial gradient is not favorable for the extremely thin foils and that, instead, the highest efficiency will be obtained by using bulk targets with a small (optimum) initial gradient. In our experiments, with the current conditions of the ultra-high contrast pulses, the pre-expansion of the carbon nanofoils appeared to be too large for efficient HOHG. Further research is required to verify these predictions in the transparent regime.

In our experiments on $\mathrm{HOHG}$ we generated high-order harmonics from nanofoils as thin as $7 \mathrm{~nm}$. These observed harmonics can be ascribed to coherent wake emission. High-order harmonics due to the relativistic oscillating mirror effect (which requires principally higher intensities but where the harmonic order is not principally limited by the plasma density) have been observed using plastic and silica targets, but only for a low number of shots. Due to the higher damage threshold of these reasonably IR-transparent targets, the formed plasma gradient may have been too steep for efficient HOHG by ROM.

When comparing the findings from the experiments and the numerical modeling on both proton acceleration and HOHG, we can conclude that, for entering the so far unexplored transparent regime with its promising advantages, it is of paramount importance to consider both the damage threshold of the target material and the pulse contrast even at the picosecond time scale. 
At the time of writing, our nanofoils and support frames are being used for novel experiments at PHI on the generation of fast ions in the regime of radiation pressure acceleration $[124,200]$. In this regime, numerical calculations and analytical models predicted that mono-energetic ions of huge energy (in the GeV-range) can be generated via this acceleration mechanism. Providing ion beams with such properties via laser-based acceleration, using the findings and approaches described in this thesis, has huge potential and is well worth further study. 
$\oplus_{\oplus}$ 


\section{A \\ CPA pulse contrast}

\section{A.1 Temporal contrast of UHI100}

At PHI, the temporal profile of the laser pulses is measured with a Sequoia third-order autocorrelator (from Amplitude Technology). The autocorrelation traces show that the ASE temporal contrast at the compressor output is typically $10^{6}$ for UHI 10 [57] and more than $10^{9}$ for UHI 100 [58]. The pulse contrast is lower on the sub-ps time scale. The initial contrast of the laser pulses can be improved by reflection on a double plasma mirror (DPM). From the previous work of Lévy [57], performed with the former UHI 10 laser system in Saclay, it is possible to infer the temporal response of the DPM and estimate the contrast of the UHI 100 system. The temporal response of the DPM is given by the simple ratio of the measured initial autocorrelation trace to the autocorrelation trace after improvement by reflection on the two plates. Due to the losses necessary to induce the reflection of the main pulse, the pulse energy is divided by a factor of 2 .

Assuming that the DPM behaves in the same way with both the UHI 10 and UHI 100 system (by applying the same fluences on both plasma mirrors and the same position of the focal spot between the two plates), we can estimate the contrast of UHI 100 plus the DPM as the simple product of the initial trace DPM response. The ASE contrast is now more than $10^{13}$. In figure A.1 the autocorrelation traces are zoomed in on a time scale of a few picoseconds. This temporal region is known as the so-called "coherent contrast" region. As can be seen from the figure, the level of the pedestals, both before and after the main pulse, is slightly higher than the ASE level. This is in general due to some misalignment of the compressor gratings. Nonetheless, it is clearly visible that with the DPM (the gray curve) the temporal profile of the laser pulse 
exhibits a very fast rising edge of about twelve orders of magnitude in less than 1 picosecond. Without DPM (the black curve), the rising edge is much less sharp and can be estimated to 9 orders of magnitude in 3 ps.

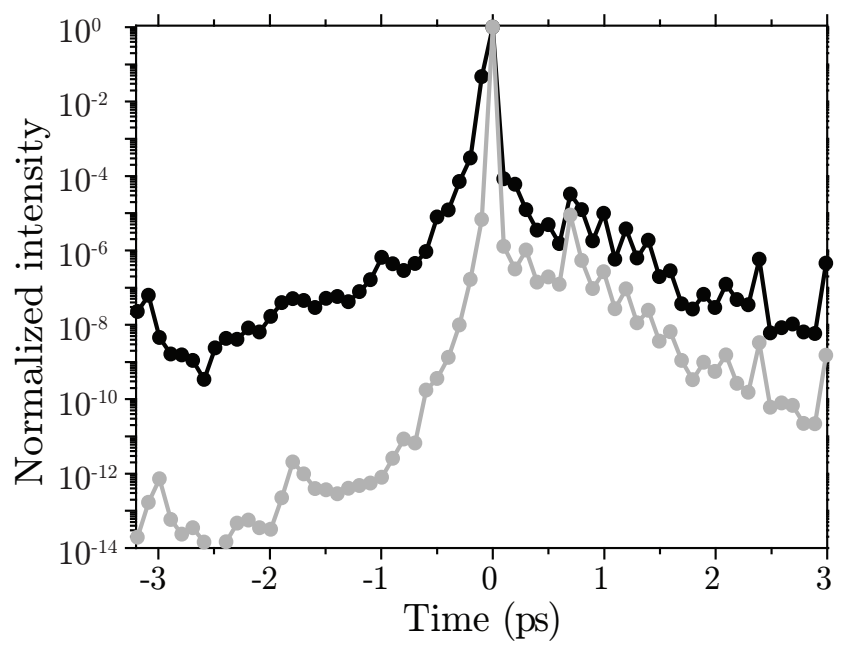

Figure A.1: Measured and estimated temporal coherent contrast for the initial pulses of the UHI 100 (black curve) and the ultra-high contrast pulses (UHI 100 plus double plasma mirror, grey curve) respectively. 


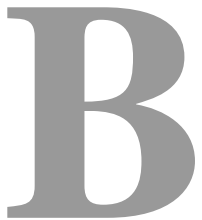

\section{Absorption coefficients}

\section{B.1 Classical collisional absorption}

The classical collisional absorption fraction for s-polarized laser light can be calculated using the formula [114]

$$
\eta_{C A}=1-e^{-\frac{8 \nu_{e i} L}{3 c} \cos ^{3} \theta} .
$$

Here $\nu_{e i}$ can be approximated by its value at the critical density, since most of the collisional absorption will take place at this density where the collision frequency is the highest. For increasing intensities, the electron quiver energy becomes comparable to the thermal velocity and an effective collision frequency [62] is used in calculations:

$$
\nu_{\mathrm{eff}}=\nu_{e i} \frac{v_{e}^{3}}{\left(v_{o s}^{2}+v_{e}^{2}\right)^{3 / 2}} .
$$

Using these equations, in the graphs in figure B.1 the classical collisional absorption fraction for laser light under normal incidence is plotted as a function of the electron thermal energy for three different scale lengths. In these examples, the target is assumed to be three-fold ionized $(Z=3)$.

For p-polarized light the analysis is more involved and the reader is referred to [62]. In the graph in figure B.2, adapted from Gibbon [62], the dependence of the absorption fraction on the angle of incidence is shown for p- and s-polarized light. 

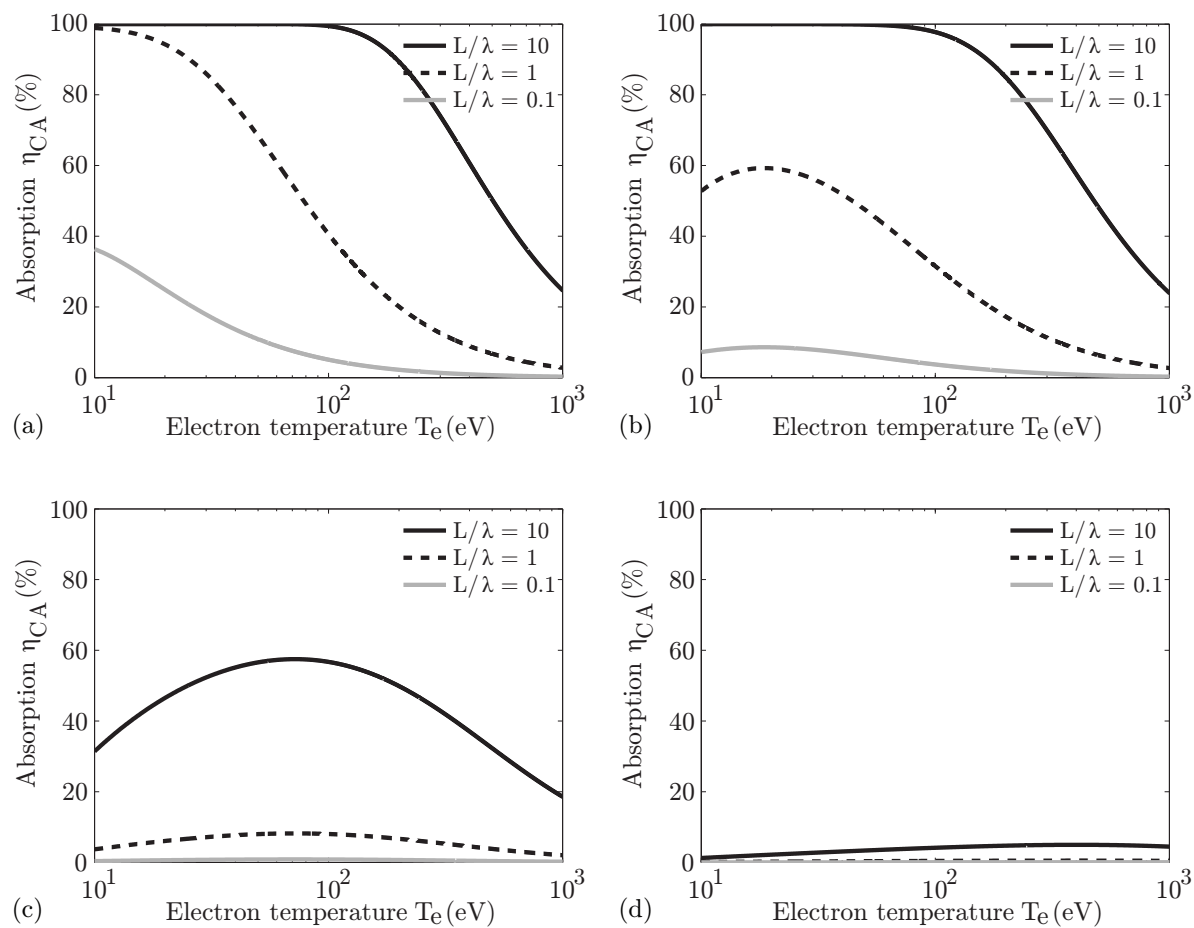

Figure B.1: Calculated classical collisional absorption fraction for four different laser intensities. (a) $I_{0}=10^{12} \mathrm{~W} / \mathrm{cm}^{2}$, (b) $I_{0}=10^{14} \mathrm{~W} / \mathrm{cm}^{2}$, (c) $I_{0}=10^{15} \mathrm{~W} / \mathrm{cm}^{2}$ and (d) $I_{0}=10^{16} \mathrm{~W} / \mathrm{cm}^{2}$.

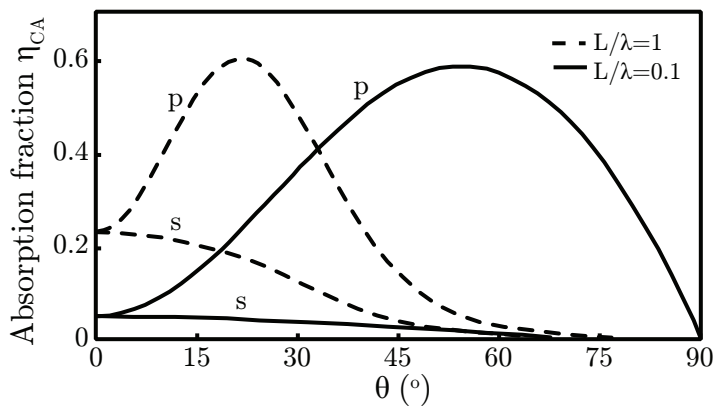

Figure B.2: Angular absorption dependence for two different density scale lengths, adapted from Gibbon [62]. The optimum absorption for p-polarized light occurs at an angle of incidence which increases as the density gradient becomes steeper. 


\section{B.2 Normal Skin Effect}

In the limit of $L \rightarrow 0$, at normal incidence, the absorption fraction for NSE becomes [62]:

$$
\eta_{N S E}= \begin{cases}\frac{2 \nu_{e i}}{\omega_{p}} & \nu_{e i} \ll \omega_{0}, \\ \frac{2 \omega_{0}}{\omega_{p}}\left(\frac{\nu_{e i}}{\omega_{0}}\right)^{1 / 2} & \nu_{e i}>\omega_{0} .\end{cases}
$$

In the graph in figure B.3, the absorption fraction is calculated for different densities, where $n_{c}$ is the critical density for radiation with a wavelength of $800 \mathrm{~nm}$.
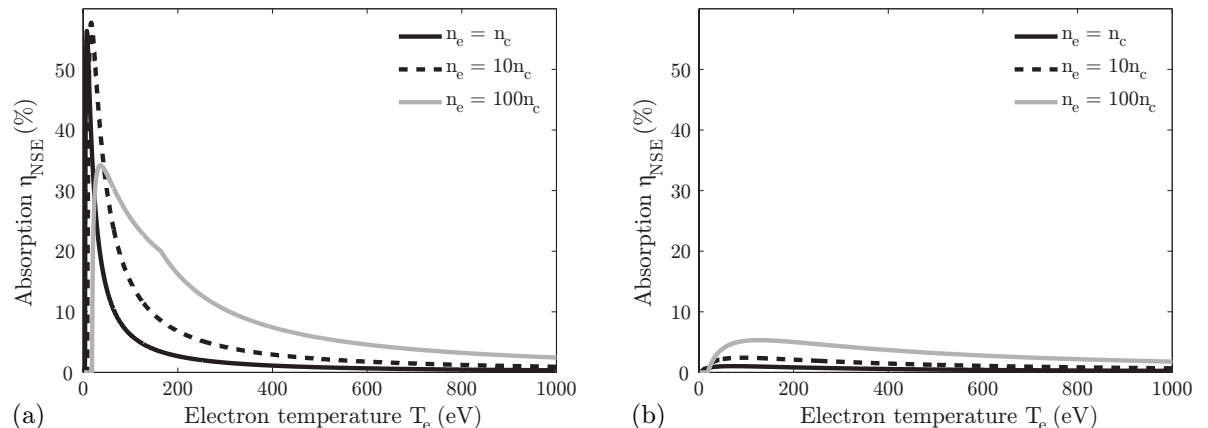

Figure B.3: Theoretical absorption fraction as due to the Normal Skin Effect for a laser intensity of (a) $I_{0}=10^{12} \mathrm{~W} / \mathrm{cm}^{2}$ and (b) $I_{0}=10^{15} \mathrm{~W} / \mathrm{cm}^{2}$. 


\section{B.3 Resonance absorption}

Theoretically, the trend of absorption fraction due to resonance absorption can be estimated by [114]

$$
\eta_{R A}=\frac{\phi^{2}(\tau)}{2}
$$

where

$$
\phi(\tau)=2.3 \tau e^{-2 \tau^{3} / 3}
$$

is the characteristic resonance function that describes the strength of the excitation as a function of the angle of incidence and the plasma density scale length, via $\tau=(k L)^{1 / 3} \sin \theta$. The peak absorption calculated by equation B.4, and shown in figure B.4, is somewhat overestimated with regard to numerical calculations and experimental measurements [114]. The maximum absorption is approximately $60 \%$, which is obtained in the limit of a long scale length [62].
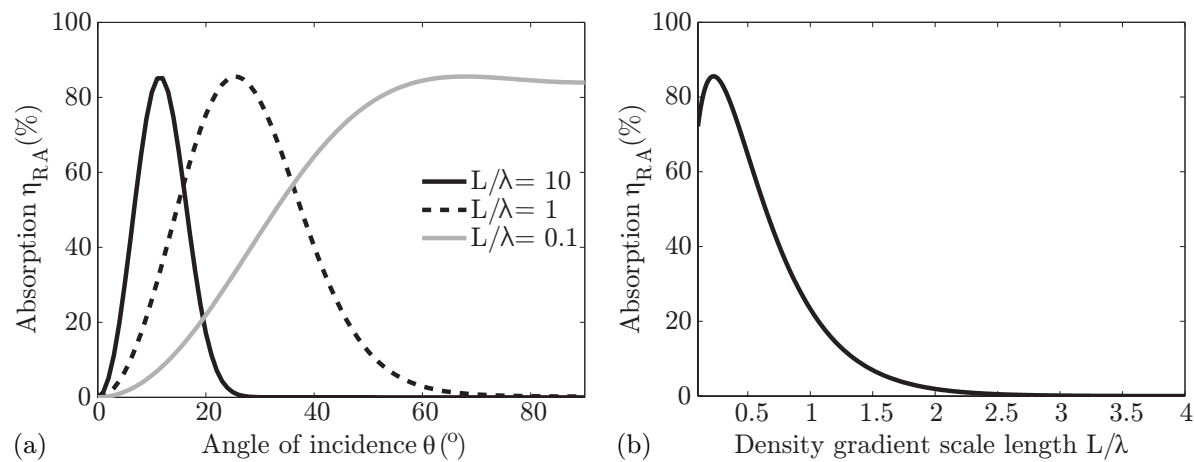

Figure B.4: Absorption fraction provided by resonance absorption for (a) three different scale lengths as a function of angle of incidence, and (b) as a function of scale length for an angle of incidence of 45 degrees. The theoretical curves are slightly overestimated. 


\section{B.4 Brunel absorption}

The absorption fraction of Brunel absorption (vacuum heating) for intensities in the non-relativistic limit $\left(a_{0}=\sqrt{I_{0} \lambda_{0 \mu}^{2} / 1.37 \cdot 10^{18}} \ll 1\right.$, see equation 2.12$)$ can be written as [62]

$$
\eta_{v h}^{\text {low }}=\frac{a_{0}}{2 \pi} f^{3} \alpha(\theta),
$$

where $\alpha(\theta)=\sin ^{3} \theta / \cos \theta$ and $f=\left[(1+8 \beta)^{1 / 2}-1\right] / 2 \beta$ with $\beta=a_{0} \alpha / 2 \pi$.

The absorption fraction for this mechanism in the relativistic $\left(a_{0} \gg 1\right)$ limit, for which the absorption fraction is independent of the intensity $\left(a_{0}\right)$, is given by:

$$
\eta_{v h}^{r e l}=\frac{4 \pi \alpha(\theta) / \sin \theta}{(\pi+\alpha(\theta) / \sin \theta)^{2}} .
$$

It can be seen that in the relativistic limit there is an optimum angle for Brunel absorption at around $70^{\circ}$.

The absorption fraction is shown in figure B.5 for three different laser intensities as a function of the angle of incidence, where the curve of $I_{0}=10^{19} \mathrm{~W} / \mathrm{cm}^{2}$ corresponds to the relativistic limit.

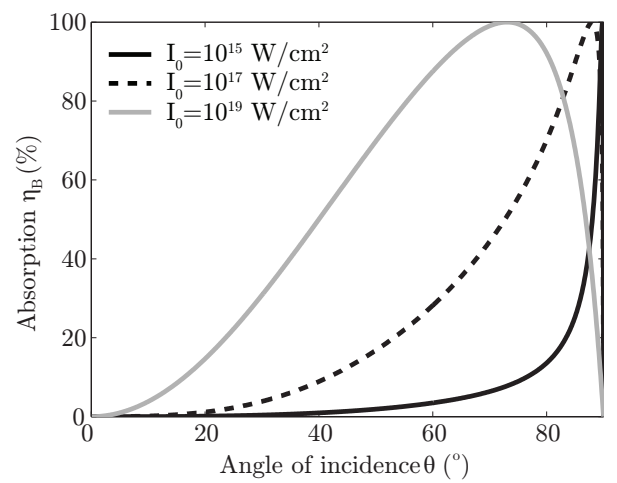

Figure B.5: Theoretical absorption fraction due to the Brunel effect for three different intensities. In the relativistic limit (grey curve) the absorption saturates. 


\section{C \\ Harmonic chirp}

\section{C.1 Harmonic chirp due to the laser pulse en- velope}

The varying envelope of the laser pulse results in a chirp of the individual harmonic orders. This chirp is not to be mistaken with a chirp in an individual attosecond pulse because an individual attosecond pulse consists of many harmonic orders. Instead, the harmonic chirp causes a variation of the temporal spacing of the attosecond pulses along the attosecond pulse train. This can be seen from the effect of the envelope on the time of emission of the high harmonics, which is illustrated in figure C.1. This example is valid for HOHG via CWE (section 3.3.2) and also for harmonics generated via HHG in gases that are due to the long trajectories (see chapter 2.2.1). The figure shows three different conditions of the electric field of the drive laser (grey curve) and the resulting emission of an attosecond pulse (black thick lines), where for illustrative purposes only a single harmonic frequency will be considered. When the laser field has a low and constant value (figure C.1a), the emission of this harmonic order occurs at a certain moment in time and the spacing in between consecutive harmonic bursts is constant. If the value of the constant laser field is higher (figure C.1b), the emission of the same harmonic frequency is slightly shifted in time with respect to the previous case, but the spacing in between the harmonic emission remains the same. Figure C.1c, shows an example of a laser pulse with a varying electric field, where the field at the beginning and end of the pulse is comparable to the situation indicated in figure C.1a), and where the peak field corresponds to the value of figure C.1b). As can be seen in the figure C.1c, the spacing in between the harmonic emission slowly increases. In the beginning of the drive pulse, the periodicity of the emission of the high 
harmonic order is somewhat shorter (blue shifted), and after the peak of the drive pulse the emission time between consecutive harmonic bursts is somewhat longer (red shifted). From Fourier analysis this means that the harmonic frequency is negatively chirped and broadened, with respect to the cases (a) and (b).

(a)

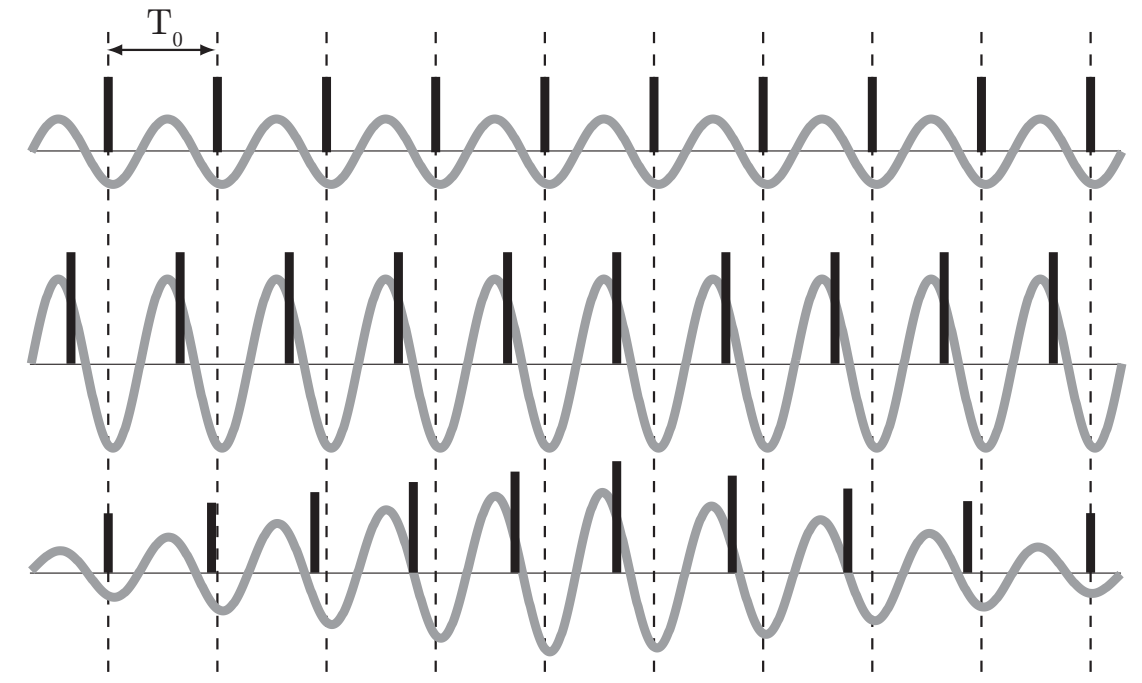

Time

Figure C.1: Intensity dependent time of harmonic emission. The electric field of the drive laser (grey curves) influences the time of emission of the harmonic pulse (black thick lines). (a) and (b) A constant drive laser field results in a constant time spacing in between consecutive harmonic pulses. A different peak value of the field results in a different time of harmonic emission. (c) A varying envelope of the drive laser results in a slowly increasing time interval in between consecutive harmonic pulses, which means that the harmonic order is broadened and negatively chirped (blue precedes red). 


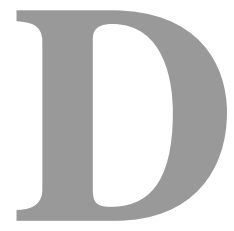

\section{Target properties}

\section{D.1 Maximum electron density and plasma fre- quency}

\begin{tabular}{lrrr}
\hline & $\rho\left(\mathrm{g} / \mathrm{cm}^{3}\right)$ & $n_{e}\left(\mathrm{~cm}^{-3}\right)$ & $w_{p}\left(w_{0}\right)$ \\
\hline Plastic foils (polystyrene) & 1.05 & $3.4 \cdot 10^{23}$ & 14.0 \\
Bulk plastic & $\approx 1.2$ & $\approx 3 \cdot 10^{23}-4 \cdot 10^{23}$ & $14-15$ \\
PET foils & 1.37 & $4.3 \cdot 10^{23}$ & 15.7 \\
Carbon foils & 2.01 & $6.0 \cdot 10^{23}$ & 18.6 \\
Bulk SiO $_{2}$ & 2.26 & $6.8 \cdot 10^{23}$ & 19.7 \\
Bulk Si & 2.33 & $7.0 \cdot 10^{23}$ & 20.0 \\
Bulk Mo & 10.2 & $2.7 \cdot 10^{24}$ & 39.3 \\
\hline
\end{tabular}

Table D.1: Mass density, maximum electron density and maximum plasma frequency of different target materials. The exact composition of bulk plastic is unknown. 


\section{Data of proton acceleration}

\section{E.1 Data extrapolation for 11 and $19 \mathrm{~nm}$ thick carbon foils}

During the experiments with the UHI 100 laser at PHI to investigate proton acceleration with the carbon nanofoils of 11 and $19 \mathrm{~nm}$ thickness, one of the pump lasers was not operational and the laser pulse energy dropped to $2.0 \mathrm{~J}$ instead of $2.5 \mathrm{~J}$. This means that the maximum ion energy can expected to be lower, based on theoretical calculated and experimentally observed scaling laws for target normal sheath acceleration (TNSA) [115]. To attempt to account for the reduced pulse energy, i.e. for an extrapolation of the proton energy expected at $2.5 \mathrm{~J}$ drive energy, we performed measurements on the dependence of the maximum proton energy on the pulse energy, the data of which is shown in figure E.1. The data at $100 \%$ corresponds to the pulse energy of $2.0 \mathrm{~J}$. The maximum proton energy at the pulse energy of $25 \%$ might have been slightly higher, due to a smaller signal-to-noise ratio from the Thomson parabola at lower drive laser energy.

Based on the generally observed scaling law of the maximum proton energy with the intensity following a square-root increase $\left(\sqrt{I_{0}}\right)$ [115], which falls in the range of the scaling laws for the hot electron temperature $\left(\left(I_{0} \lambda_{0}^{2}\right)^{1 / 3}\right.$ to $\left(I_{0} \lambda_{0}^{2}\right)^{3 / 4}$, as discussed at the end of section 3.1.3), a fit was made to the experimental data with $y=a \cdot x^{0.5}$ using a least squares curve fitting (LevenbergMarquardt algorithm in the software program Origin). The experimental values of the maximum proton energy can be fitted well with a square-root increase with the energy and thus of the intensity. In the graph, the curve (solid line) is plotted with a square-root fitting. To rule out any effect of a possible systematical error in the recorded data, a second fit was made with a shifted origin 
( $y=a \cdot x^{0.5}+b$, dashed curve), this resulted in an almost identical extrapolated value at $125 \%$. Using the square-root fitting, in figure 5.10 , the maximum proton energy was extrapolated for the case when a pulse energy of $2.5 \mathrm{~J}$ was used with the 11 and $19 \mathrm{~nm}$ thick foils.

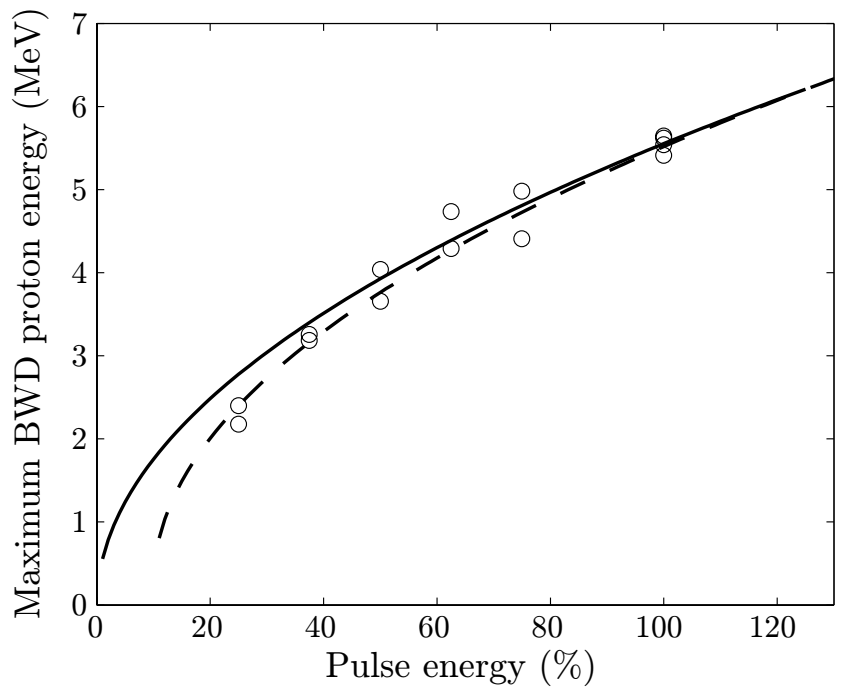

Figure E.1: Maximum proton energy as a function of the laser pulse energy (2.0 J corresponds to $100 \%$ ) for a carbon foil of $11 \mathrm{~nm}$ thick. The solid curve is a fit with a $\sqrt{I_{0}}$-function. The dashed curve is similar fit but a shifted in origin. 


\section{Evolution of electron density}

From numerical calculations one can obtain the electron density and its evolution. Calculations were made on the highly complex interaction of four different plasma profiles with a $\sin ^{2}$-shaped laser pulse (not visible) of $10 \mathrm{fs}$ (FWHM) and $a_{0}=5\left(I_{0}=5.4 \cdot 10^{19} \mathrm{~W} / \mathrm{cm}^{2}\right)$, which is incident from the left under an angle of $45^{\circ}$. The laser pulse interacts with the plasma during the time from 3 to 12 (in units of $2 \pi / \omega$ ). The plasma profiles are a bulk target with a step-like profile, a bulk target with an exponential gradient of $L / \lambda=0.1$, a foil target with a step-like profile and a foil target with an exponential gradient of $L / \lambda=0.1$ on both sides. The initial maximum electron density is $110 n_{c}$ and scaled for the expanded foil in order to conserve the number of electrons in the foil. In the following four figures F.1 to F.4, the electron density from the respectively mentioned plasma profiles is plotted in a logarithmic color scale as a function of space (position $\mathrm{x}$ ) and time. Note the different scale of the horizontal axes for the bulk and foil targets. From the figures it can be seen that some electrons are pulled into the vacuum (on the left side) and are pushed back into the bulk plasma or through the foil plasma. The density of these electrons and the excursion amplitude depends on the target profile. A description and comparison of the figures is presented in the main text in chapter 5 . 


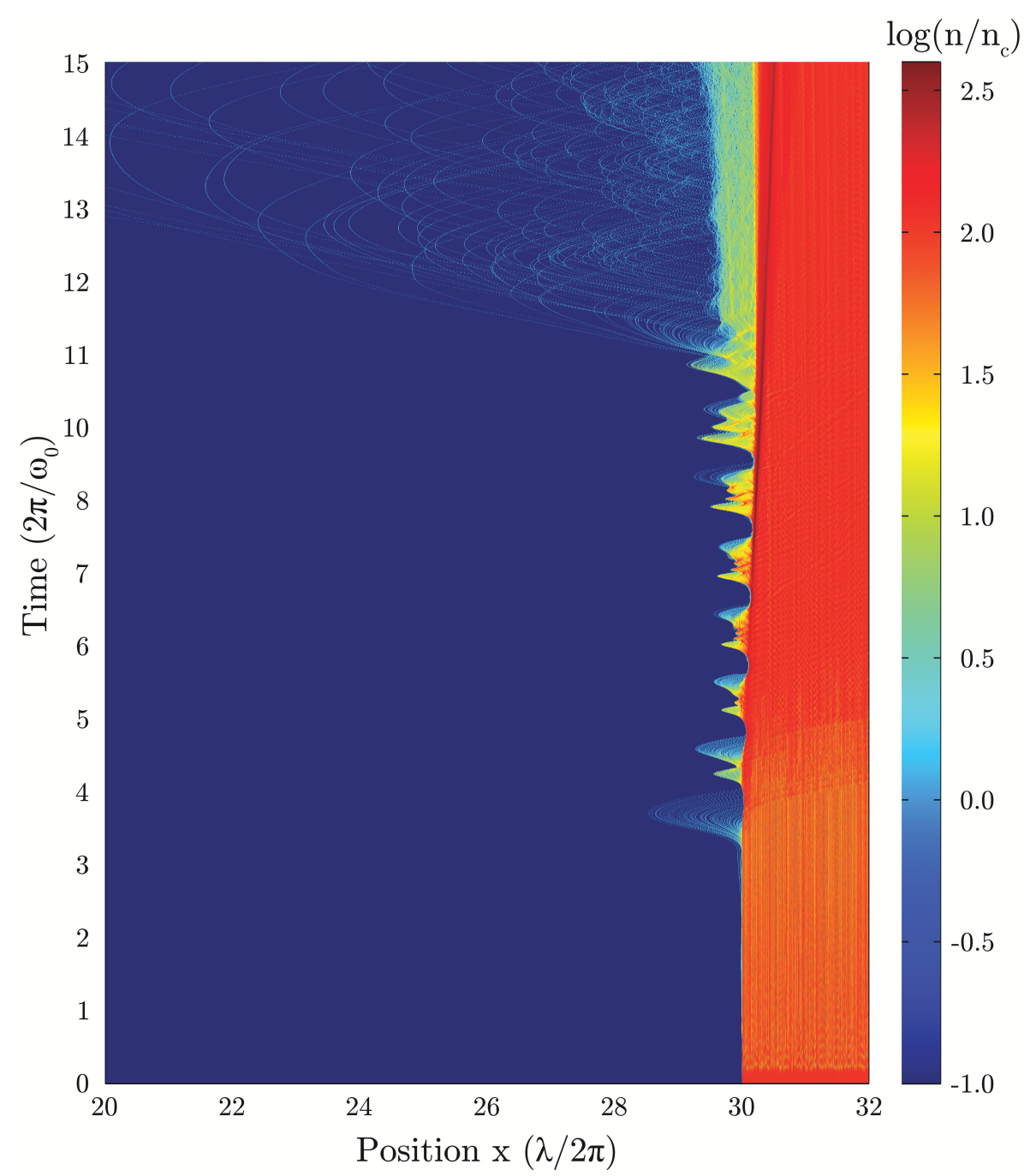

Figure F.1: The evolution of the electron density in a logarithmic color scale in space and time for a bulk target initially with a step-like profile. 


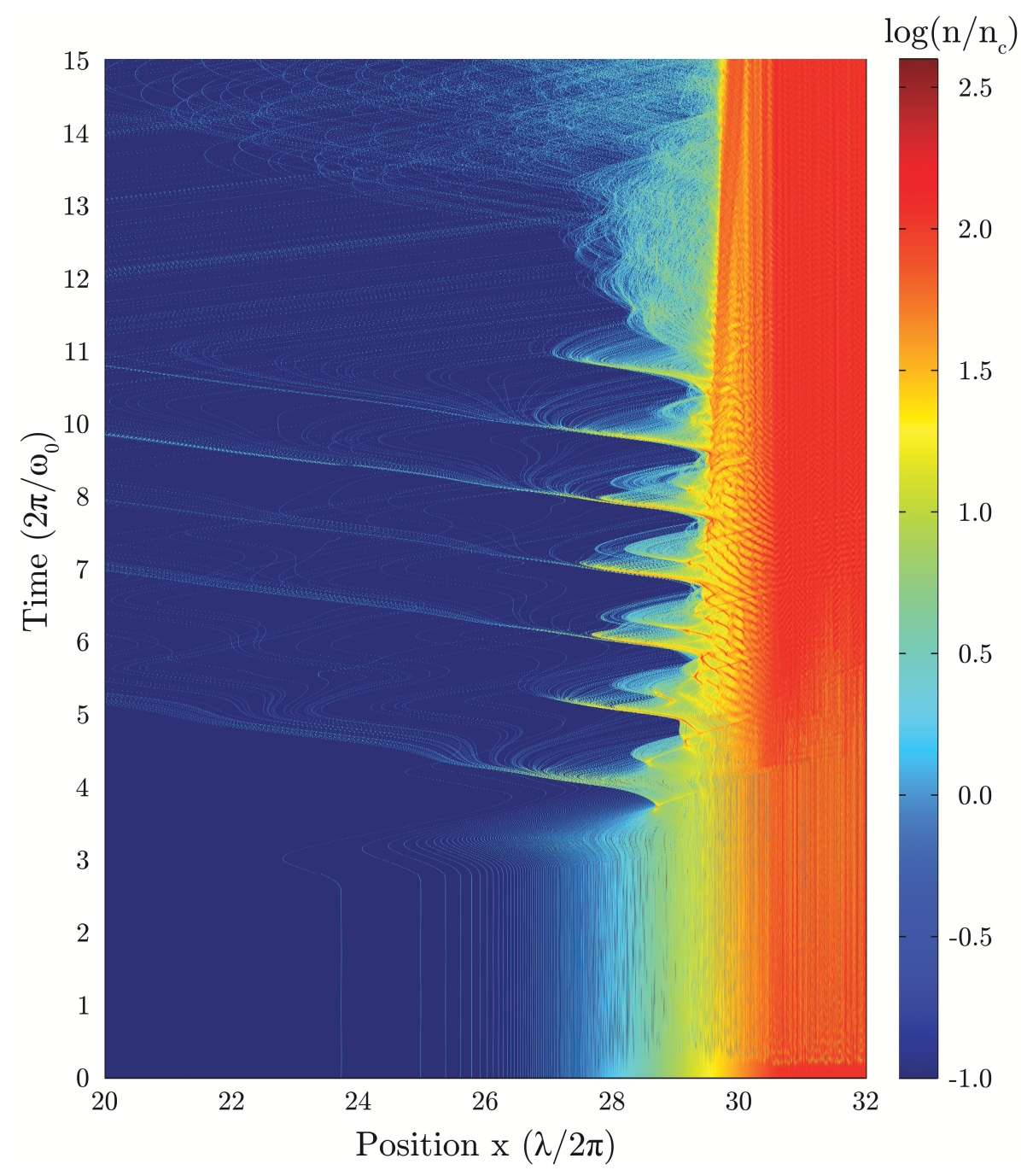

Figure F.2: The evolution of the electron density in a logarithmic color scale in space and time for a bulk target initially with a gradient profile with a scale length of $L / \lambda=0.1$. 


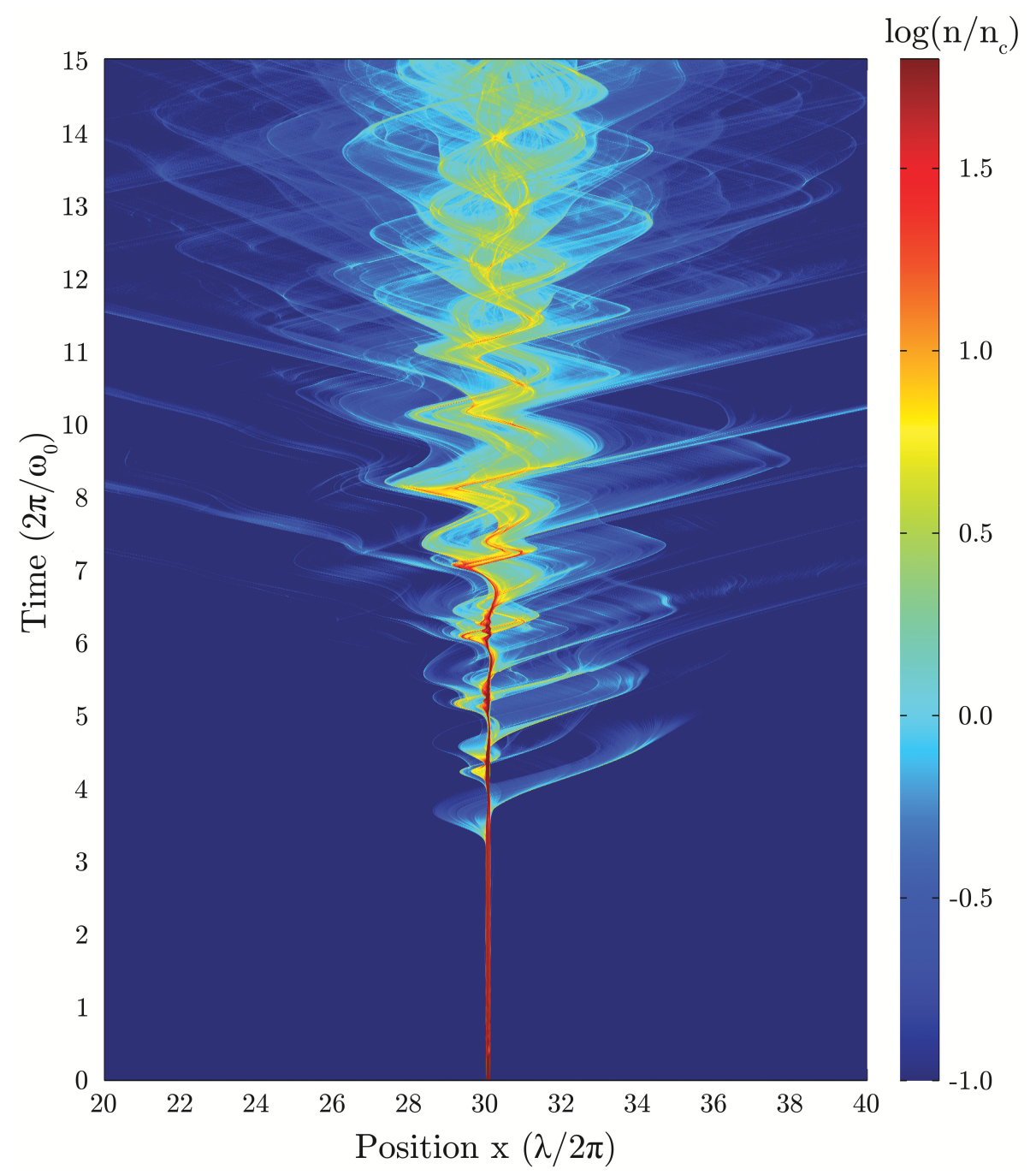

Figure F.3: The evolution of the electron density in a logarithmic color scale in space and time for a foil target (thickness of $0.1 \lambda / 2 \pi$ ) initially with a step-like profile. 


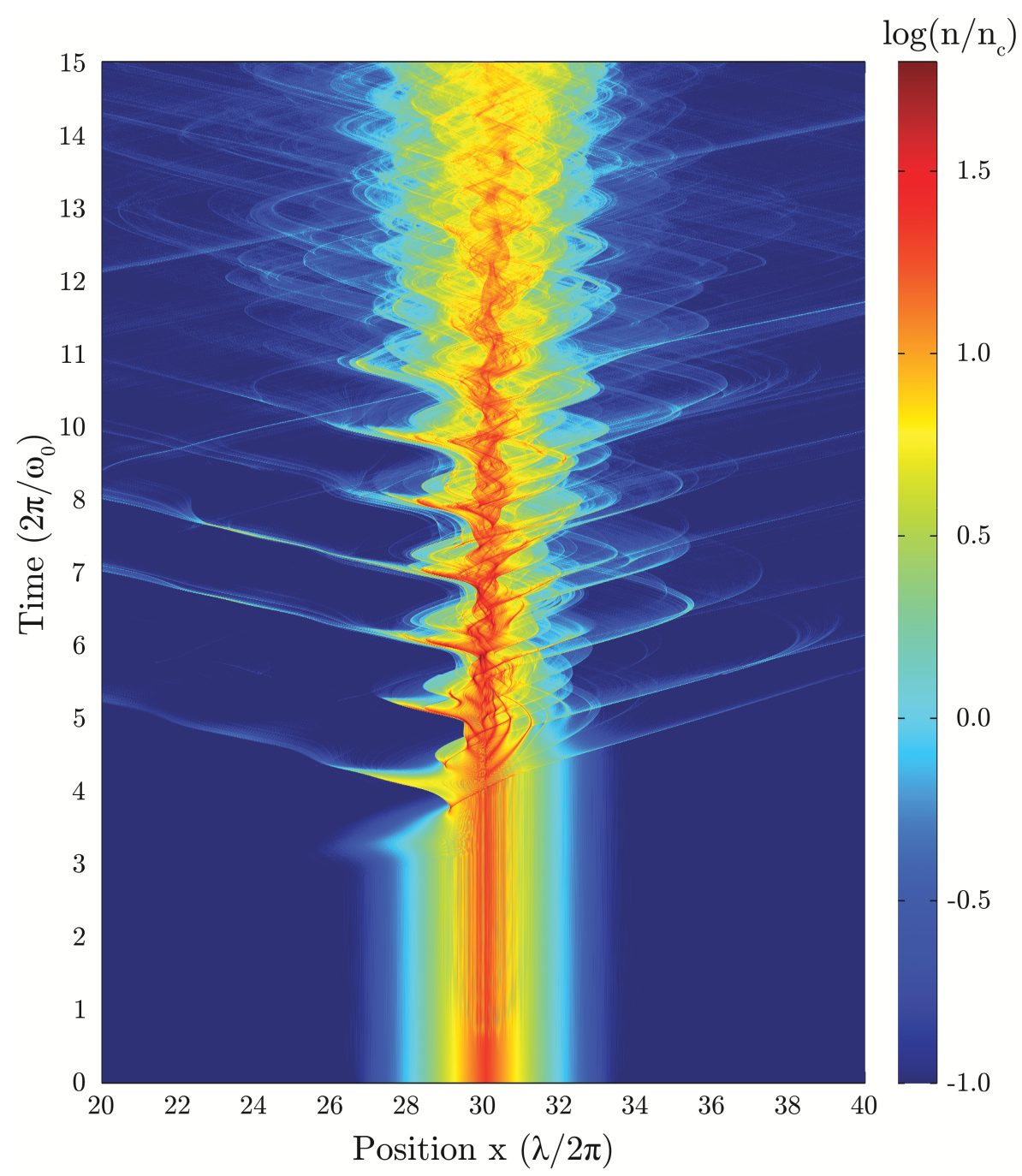

Figure F.4: The evolution of the electron density in a logarithmic color scale in space and time for a foil target (thickness of $0.1 \lambda / 2 \pi$ ) initially with a gradient profile with a scale length of $L / \lambda=0.1$. 


\section{Bibliography}

[1] H.-P. Berlien and G. J. Mueller, Applied Laser Medicine (Springer, Berlin, 2003).

[2] M. Tabak, D. S. Clark, S. P. Hatchett, M. H. Key, B. F. Lasinski, R. A. Snavely, S. C. Wilks, R. P. J. Town, R. Stephens, E. M. Campbell, R. Kodama, K. Mima, K. A. Tanaka, S. Atzeni, and R. Freeman, "Review of progress in Fast Ignition," Physics of Plasmas 12, 057 305-8 (2005).

[3] G. Mejean, J. Kasparian, J. Yu, S. Frey, E. Salmon, and J. P. Wolf, "Remote detection and identification of biological aerosols using a femtosecond terawatt lidar system," Applied Physics B: Lasers and Optics 78, 535-537 (2004).

[4] D. Umstadter, "Review of physics and applications of relativistic plasmas driven by ultra-intense lasers," in The 42nd annual meeting of the division of plasma physics of the American Physical Society and the 10th international congress on plasma physics 8, 1774-1785 (2001).

[5] A. Pukhov, "Strong field interaction of laser radiation," Reports on Progress in Physics 66, 47-101 (2003).

[6] K. W. D. Ledingham, P. McKenna, and R. P. Singhal, "Applications for Nuclear Phenomena Generated by Ultra-Intense Lasers," Science 300, 1107-1111 (2003).

[7] T. Ditmire, S. Bless, G. Dyer, A. Edens, W. Grigsby, G. Hays, K. Madison, A. Maltsev, J. Colvin, M. J. Edwards, R. W. Lee, P. Patel, D. Price, B. A. Remington, R. Sheppherd, A. Wootton, J. Zweiback, E. Liang, and K. A. Kielty, "Overview of future directions in high energy-density and high-field science using ultra-intense lasers," Radiation Physics and Chemistry 70, 535-552 (2004).

[8] K. Krushelnick, E. L. Clark, F. N. Beg, A. E. Dangor, Z. Najmudin, P. A. Norreys, M. Wei, and M. Zepf, "High intensity laser-plasma sources of ions - physics and future applications," Plasma Physics and Controlled Fusion 47, B451 (2005).

[9] L. Robson, P. McKenna, T. McCanny, K. W. D. Ledingham, J. M. Gillies, and J. Zweit, "High-Power Laser Production of PET Isotopes," in Lasers and Nuclei (2006), pp. 191-203. 
[10] C. Ma, I. Veltchev, E. Fourkal, J. Li, W. Luo, J. Fan, T. Lin, and A. Pollack, "Development of a laser-driven proton accelerator for cancer therapy," Laser Physics 16, 639-646 (2006).

[11] H. Wille, M. Rodriguez, J. Kasparian, D. Mondelain, J. Yu, A. Mysyrowicz, R. Sauerbrey, J. P. Wolf, and L. Woste, "Teramobile: A mobile femtosecond-terawatt laser and detection system," The European Physical Journal Applied Physics 20, 183-190 (2002).

[12] V. Malka, J. Faure, Y. A. Gauduel, E. Lefebvre, A. Rousse, and K. T. Phuoc, "Principles and applications of compact laser-plasma accelerators," Nature Physics 4, 447-453 (2008).

[13] T. H. Maiman, "Optical and Microwave-Optical Experiments in Ruby," Physical Review Letters 4, 564 (1960).

[14] P. A. Franken, A. E. Hill, C. W. Peters, and G. Weinreich, "Generation of Optical Harmonics," Physical Review Letters 7, 118 (1961).

[15] D. E. Spence, P. N. Kean, and W. Sibbett, "60-fsec pulse generation from a self-mode-locked Ti:sapphire laser," Optics Letters 16, 42-44 (1991).

[16] D. Strickland and G. Mourou, "Compression of amplified chirped optical pulses," Optics Communications 56, 219-221 (1985).

[17] S. Backus, C. G. Durfee III, M. M. Murnane, and H. C. Kapteyn, "High power ultrafast lasers," Review of Scientific Instruments 69, 1207-1223 (1998).

[18] G. A. Mourou, T. Tajima, and S. V. Bulanov, "Optics in the relativistic regime," Reviews of Modern Physics 78, 309-63 (2006).

[19] J. G. Eden, "High-order harmonic generation and other intense optical field-matter interactions: review of recent experimental and theoretical advances," Progress in Quantum Electronics 28, 197-246 (2004).

[20] C. Thaury, F. Quere, J. P. Geindre, A. Levy, T. Ceccotti, P. Monot, M. Bougeard, F. Reau, P. D'Oliveira, P. Audebert, R. Marjoribanks, and P. Martin, "Plasma mirrors for ultrahigh-intensity optics," Nature Physics 3, 424-429 (2007).

[21] J. Faure, Y. Glinec, A. Pukhov, S. Kiselev, S. Gordienko, E. Lefebvre, J. P. Rousseau, F. Burgy, and V. Malka, "A laser-plasma accelerator producing monoenergetic electron beams," Nature 431, 541-544 (2004).

[22] A. G. Khachatryan, "Trapping, compression, and acceleration of an electron bunch in the nonlinear laser wakefield," Physical Review E 65, 046504 (2002). 
[23] H. Schwoerer, S. Pfotenhauer, O. Jackel, K. U. Amthor, B. Liesfeld, W. Ziegler, R. Sauerbrey, K. W. D. Ledingham, and T. Esirkepov, "Laserplasma acceleration of quasi-monoenergetic protons from microstructured targets," Nature 439, 445-448 (2006).

[24] E. Surdutovich and A. V. Solov'yov, "A physical palette for ion-beam cancer therapy," Europhysics News 40, 21-24 (2009).

[25] G. Kraft and S. D. Kraft, "Research needed for improving heavy-ion therapy," New Journal of Physics 11, 025001 (2009).

[26] M. Dunne, "APPLIED PHYSICS: Laser-Driven Particle Accelerators," Science 312, 374-376 (2006).

[27] J. Fuchs, P. Audebert, P. Antici, E. Brambrink, E. d'Humieres, J.-C. Gauthier, and L. Romagnani, "Review of high-brightness proton and ion acceleration using pulsed lasers," in 39 $9^{\text {th }}$ ICFA Advanced Beam Dynamics Workshop - High Intensity High Brightness Hadron Beams pp. 319-323 (2006).

[28] M. Borghesi, D. H. Campbell, A. Schiavi, M. G. Haines, O. Willi, A. J. MacKinnon, P. Patel, L. A. Gizzi, M. Galimberti, R. J. Clarke, F. Pegoraro, H. Ruhl, and S. Bulanov, "Electric field detection in laser-plasma interaction experiments via the proton imaging technique," in Review, Tutorial and Invited Papers from the 43rd Annual Meeting of the APS Division of Plasma Physics 9, 2214-2220 (2002).

[29] U. Linz and J. Alonso, "What will it take for laser driven proton accelerators to be applied to tumor therapy?" Physical Review Special Topics - Accelerators and Beams 10, 094801 (2007).

[30] M. Uiberacker, T. Uphues, M. Schultze, A. J. Verhoef, V. Yakovlev, M. F. Kling, J. Rauschenberger, N. M. Kabachnik, H. Schroder, M. Lezius, K. L. Kompa, H. G. Muller, M. J. J. Vrakking, S. Hendel, U. Kleineberg, U. Heinzmann, M. Drescher, and F. Krausz, "Attosecond real-time observation of electron tunnelling in atoms," Nature 446, 627-632 (2007).

[31] N. Anscombe and F. Krausz, "Attosecond analysis," Nature Photonics 2, 548-548 (2008).

[32] G. D. Tsakiris, K. Eidmann, J. Meyer-ter Vehn, and F. Krausz, "Route to intense single attosecond pulses," New Journal of Physics 8, 19 (2006).

[33] D. Umstadter, "Relativistic laser-plasma interactions," Journal of Physics D: Applied Physics 36, R151-R165 (2003).

[34] A. H. Zewail, "Femtochemistry: Atomic-Scale Dynamics of the Chemical Bond," The Journal of Physical Chemistry A 104, 5660-5694 (2000). 
[35] M. Hentschel, R. Kienberger, C. Spielmann, G. A. Reider, N. Milosevic, T. Brabec, P. Corkum, U. Heinzmann, M. Drescher, and F. Krausz, "Attosecond metrology," Nature 414, 509-513 (2001).

[36] Y. Silberberg, "Laser science: Physics at the attosecond frontier," Nature 414, 494-495 (2001).

[37] M. Wieland, C. Spielmann, U. Kleineberg, T. Westerwalbesloh, U. Heinzmann, and T. Wilhein, "Toward time-resolved soft X-ray microscopy using pulsed fs-high-harmonic radiation," Ultramicroscopy 102, 93-100 (2005).

[38] R. L. Sandberg, A. Paul, D. A. Raymondson, S. Hadrich, D. M. Gaudiosi, J. Holtsnider, R. I. Tobey, O. Cohen, M. M. Murnane, H. C. Kapteyn, C. Song, J. Miao, Y. Liu, and F. Salmassi, "Lensless Diffractive Imaging Using Tabletop Coherent High-Harmonic Soft-X-Ray Beams," Physical Review Letters 99, 098 103-4 (2007).

[39] S. Dobosz, G. Doumy, H. Stabile, P. D'Oliveira, P. Monot, F. Reau, S. Huller, and P. Martin, "Probing Hot and Dense Laser-Induced Plasmas with Ultrafast XUV Pulses," Physical Review Letters 95, 025 001-4 (2005).

[40] H. H. Solak and Y. Ekinci, "Bit-array patterns with density over 1 Tbit/in. ${ }^{2}$ fabricated by extreme ultraviolet interference lithography," $\mathbf{2 5}$, 2123-2126 (2007).

[41] V. Auzelyte, C. Dais, P. Farquet, D. Grutzmacher, L. J. Heyderman, F. Luo, S. Olliges, C. Padeste, P. K. Sahoo, T. Thomson, A. Turchanin, C. David, and H. H. Solak, "Extreme ultraviolet interference lithography at the Paul Scherrer Institut [Abstract]," Journal of Micro/Nanolithography, MEMS and MOEMS 8, 021 204-10 (2009).

[42] A. R. Libertun, X. Zhang, A. Paul, E. Gagnon, T. Popmintchev, S. Backus, M. M. Murnane, H. C. Kapteyn, and I. P. Christov, "Design of fully spatially coherent extreme-ultraviolet light sources," Applied Physics Letters 84, 3903-3905 (2004).

[43] G. Lambert, T. Hara, D. Garzella, T. Tanikawa, M. Labat, B. Carre, H. Kitamura, T. Shintake, M. Bougeard, S. Inoue, Y. Tanaka, P. Salieres, H. Merdji, O. Chubar, O. Gobert, K. Tahara, and M. E. Couprie, "Injection of harmonics generated in gas in a free-electron laser providing intense and coherent extreme-ultraviolet light," Nature Physics 4, 296300 (2008).

[44] T. Sekikawa, A. Kosuge, T. Kanai, and S. Watanabe, "Nonlinear optics in the extreme ultraviolet," Nature 432, 605-608 (2004). 
[45] F. Gruner, S. Becker, U. Schramm, T. Eichner, M. Fuchs, R. Weingartner, D. Habs, J. Meyer-ter Vehn, M. Geissler, M. Ferrario, L. Serafini, B. van der Geer, H. Backe, W. Lauth, and S. Reiche, "Design considerations for table-top, laser-based VUV and X-ray free electron lasers," Applied Physics B: Lasers and Optics 86, 431-435 (2007).

[46] A. Irman, M. J. H. Luttikhof, A. G. Khachatryan, F. A. van Goor, J. W. J. Verschuur, H. M. J. Bastiaens, and K. J. Boller, "Design and simulation of laser wakefield acceleration with external electron bunch injection in front of the laser pulse," Journal of Applied Physics 102, 024 513-7 (2007).

[47] A. Irman, Integral design of a laser wakefield accelerator with external bunch injection, Ph.D. thesis, University of Twente (2009).

[48] I. Nedelcu, R. W. E. van de Kruijs, A. E. Yakshin, G. von Blanckenhagen, and F. Bijkerk, "Reflectivity and surface roughness of multilayer-coated substrate recovery layers for EUV lithographic optics," Optical Engineering 47, 063 801-5 (2008).

[49] A. Paul, E. A. Gibson, Z. Xiaoshi, A. Lytle, T. Popmintchev, Z. Xibin, M. M. Murnane, I. P. Christov, and H. C. Kapteyn, "Phase-matching techniques for coherent soft X-ray generation," Quantum Electronics, IEEE Journal of 42, 14-26 (2006).

[50] C. Winterfeldt, C. Spielmann, and G. Gerber, "Colloquium: Optimal control of high-harmonic generation," Reviews of Modern Physics 80, 117-24 (2008).

[51] B. Dromey, M. Zepf, M. Landreman, and S. M. Hooker, "Quasiphasematching of harmonic generation via multimode beating in waveguides," Optics Express 15, 7894-7900 (2007).

[52] Q. L. Dong, Z. M. Sheng, M. Y. Yu, and J. Zhang, "Optimization of ion acceleration in the interaction of intense femtosecond laser pulses with ultrathin foils," Physical Review E (Statistical, Nonlinear, and Soft Matter Physics) 68, 026408 (2003).

[53] E. Collett and E. Wolf, "Is complete spatial coherence necessary for the generation of highly directional light beams?" Optics Letters 2, 27 (1978).

[54] H. C. Kapteyn and M. M. Murnane, "Prepulse energy suppression for high-energy ultrashort pulses using self-induced plasma shuttering," Optics Letters 16, 490-492 (1991).

[55] G. Doumy, F. Quere, O. Gobert, M. Perdrix, P. Martin, P. Audebert, J. C. Gauthier, J. P. Geindre, and T. Wittmann, "Complete characterization of a plasma mirror for the production of high-contrast ultraintense 
laser pulses," Physical Review E (Statistical, Nonlinear, and Soft Matter Physics) 69, 026 402-12 (2004).

[56] B. Dromey, S. Kar, M. Zepf, and P. Foster, "The plasma mirror-A subpicosecond optical switch for ultrahigh power lasers," Review of Scientific Instruments 75, 645-649 (2004).

[57] A. Levy, T. Ceccotti, P. D’Oliveira, F. Reau, M. Perdix, F. Quere, P. Monot, M. Bougeard, H. Lagadec, P. Martin, J.-P. Geindre, and P. Audebert, "Double plasma mirror for ultrahigh temporal contrast ultraintense laser pulses," Optics Letters 32, 310-312 (2007).

[58] R. A. Loch, P. Martin, T. Ceccotti, P. Monot, F. Quere, H. George, M. Bougeard, F. Reau, P. D'Oliveira, and K.-J. Boller, "High-order harmonic and fast ion generation in high intensity laser-solid interactions," in Second International Symposium on Laser-Driven Relativistic Plasmas Applied to Science, Industry and Medicine (2009), accepted for publication.

[59] E. J. Takahashi, T. Kanai, K. L. Ishikawa, Y. Nabekawa, and K. Midorikawa, "Dramatic Enhancement of High-Order Harmonic Generation," Physical Review Letters 99, 053 904-4 (2007).

[60] F. F. Chen, Introduction to plasma physics (Plenum Press, New York, 1974).

[61] D. Attwood, Soft X-Rays and Extreme Ultraviolet Radiations : Principles and Applications (Cambridge University Press, Cambridge, UK, 2000).

[62] P. Gibbon, Short Pulse Laser Interactions with Matter - An Introduction (Imperial College Press, London, 2005).

[63] S. M. Mominuzzaman, K. M. Krishna, T. Soga, T. Jimbo, and M. Umeno, "Optical Absorption and Electrical Conductivity of Amorphous Carbon Thin Films from Camphor: A Natural Source," Japanese Journal of Applied Physics 38, 658-663 (1999).

[64] S. C. Wilks and W. L. Kruer, "Absorption of ultrashort, ultra-intense laser light by solids and overdense plasmas," Quantum Electronics, IEEE Journal of 33, 1954-1968 (1997).

[65] M. Protopapas, C. Keitel, and P. Knight, "Atomic physics with superhigh intensity lasers," Reports on Progress in Physics 60, 389 (1997).

[66] T. Pfeifer, C. Spielmann, and G. Gerber, "Femtosecond x-ray science," Reports on Progress in Physics 69, 443 (2006).

[67] C. A. Froud, Designing a Nanoscale X-ray Source: Towards Single Molecule X-ray Scattering, Ph.D. thesis, University of Southampton (2007). 
[68] E. A. Gibson, Quasi-Phase Matching of Soft X-ray Light from High-Order Harmonic Generation using Waveguide Structures, Ph.D. thesis, University of Colorado (2004).

[69] C. Winterfeldt, Generation and control of high-harmonic radiation, Ph.D. thesis, University of Wuerzburg (2006).

[70] A. J. Paul, Coherent EUV Light from High-Order Harmonic Generation: Enhancement and Applications to Lensless Diffractive Imaging, Ph.D. thesis, University of Colorado (2007).

[71] P. B. Corkum, "Plasma perspective on strong field multiphoton ionization," Physical Review Letters 71, 1994 (1993).

[72] P. B. Corkum and F. Krausz, "Attosecond science," Nature Physics 3, 381-387 (2007).

[73] M. Lewenstein, P. Balcou, M. Y. Ivanov, A. L'Huillier, and P. B. Corkum, "Theory of high-harmonic generation by low-frequency laser fields," Physical Review A 49, 2117 (1994).

[74] M. Lewenstein, P. Salieres, and A. L'Huillier, "Phase of the atomic polarization in high-order harmonic generation," Physical Review A 52, 4747 (1995).

[75] E. Seres, J. Seres, and C. Spielmann, "X-ray absorption spectroscopy in the keV range with laser generated high harmonic radiation," Applied Physics Letters 89, 181 919-3 (2006).

[76] I. Christov, H. Kapteyn, and M. Murnane, "Quasi-phase matching of high-harmonics and attosecond pulses in modulated waveguides," Optics Express 7, 362-367 (2000).

[77] R. R. Freeman, P. H. Bucksbaum, and T. J. McIlrath, "The ponderomotive potential of high intensity light and its role in the multiphoton ionization of atoms," Quantum Electronics, IEEE Journal of 24, 14611469 (1988).

[78] P. Balcou, P. Salieres, A. L'Huillier, and M. Lewenstein, "Generalized phase-matching conditions for high harmonics: The role of field-gradient forces," Physical Review A 55, 3204 (1997).

[79] M. B. Gaarde, F. Salin, E. Constant, P. Balcou, K. J. Schafer, K. C. Kulander, and A. L'Huillier, "Spatiotemporal separation of high harmonic radiation into two quantum path components," Physical Review A 59, 1367 (1999).

[80] K. Varju, Y. Mairesse, B. Carr, M. B. Gaarde, P. Johnsson, S. Kazamias, R. Lopez-Martens, J. Mauritsson, K. J. Schafer, P. H. Balcou, A. L'Huillier, and P. Salieres, "Frequency chirp of harmonic and attosecond pulses," Journal of Modern Optics 52, 379-394 (2005). 
[81] R. A. Bartels, A. Paul, H. Green, H. C. Kapteyn, M. M. Murnane, S. Backus, I. P. Christov, Y. Liu, D. Attwood, and C. Jacobsen, "Generation of Spatially Coherent Light at Extreme Ultraviolet Wavelengths," Science 297, 376-378 (2002).

[82] J. Biegert, A. Heinrich, C. P. Hauri, W. Kornelis, P. Schlup, M. Anscombe, K. J. Schafer, M. B. Gaarde, and U. Keller, "Enhancement of High-Order Harmonic Emission Using Attosecond Pulse Trains," Laser Physics 15, 899-902 (2005).

[83] C. G. Durfee, A. R. Rundquist, S. Backus, C. Herne, M. M. Murnane, and H. C. Kapteyn, "Phase Matching of High-Order Harmonics in Hollow Waveguides," Physical Review Letters 83, 2187 (1999).

[84] A. N. Naumov, A. M. Zheltikov, A. B. Fedotov, D. A. Sidorov-Biryukov, A. P. Tarasevitch, P. Zhou, and D. von der Linde, "Pressure control of phase matching in high-order harmonic generation in hollow fibers filled with an absorbing weakly ionizing gas," Journal of the Optical Society of America B 18, 811-817 (2001).

[85] A. Rundquist, I. Durfee, Charles G., Z. Chang, C. Herne, S. Backus, M. M. Murnane, and H. C. Kapteyn, "Phase-Matched Generation of Coherent Soft X-rays," Science 280, 1412-1415 (1998).

[86] P. Salieres and M. Lewenstein, "Generation of ultrashort coherent XUV pulses by harmonic conversion of intense laser pulses in gases: towards attosecond pulses," Measurement Science and Technology 12, 1818 (2001).

[87] E. Constant, D. Garzella, P. Breger, E. Mevel, C. Dorrer, C. Le Blanc, F. Salin, and P. Agostini, "Optimizing High Harmonic Generation in Absorbing Gases: Model and Experiment," Physical Review Letters 82, 1668 (1999).

[88] S. Kim, J. Jin, Y.-J. Kim, I.-Y. Park, Y. Kim, and S.-W. Kim, "Highharmonic generation by resonant plasmon field enhancement," Nature 453, 757-760 (2008).

[89] T. Popmintchev, M.-C. Chen, O. Cohen, M. E. Grisham, J. J. Rocca, M. M. Murnane, and H. C. Kapteyn, "Extended phase matching of high harmonics driven by mid-infrared light," Optics Letters 33, 2128-2130 (2008).

[90] A. L. Lytle, X. Zhang, R. L. Sandberg, O. Cohen, H. C. Kapteyn, and M. M. Murnane, "Quasi-phase matching and characterization of highorder harmonic generation in hollow waveguides using counterpropagating light," Opt. Express 16, 6544-6566 (2008).

[91] J. Tate, T. Auguste, H. G. Muller, P. Salieres, P. Agostini, and L. F. DiMauro, "Scaling of Wave-Packet Dynamics in an Intense Midinfrared Field," Physical Review Letters 98, 013 901-4 (2007). 
[92] E. A. Gibson, A. Paul, N. Wagner, R. Tobey, S. Backus, I. P. Christov, M. M. Murnane, and H. C. Kapteyn, "High-Order Harmonic Generation up to $250 \mathrm{eV}$ from Highly Ionized Argon," Physical Review Letters 92, 033001 (2004).

[93] B. A. Reagan, T. Popmintchev, M. E. Grisham, D. M. Gaudiosi, M. Berrill, O. Cohen, B. C. Walker, M. M. Murnane, J. J. Rocca, and H. C. Kapteyn, "Enhanced high-order harmonic generation from Xe, Kr, and Ar in a capillary discharge," Physical Review A (Atomic, Molecular, and Optical Physics) 76, 013 816-10 (2007).

[94] D. M. Gaudiosi, B. Reagan, T. Popmintchev, M. Grisham, M. Berrill, O. Cohen, B. C. Walker, M. M. Murnane, H. C. Kapteyn, and J. J. Rocca, "High-Order Harmonic Generation from Ions in a Capillary Discharge," Physical Review Letters 96, 203001-4 (2006).

[95] K. Ishikawa, "Photoemission and Ionization of $\mathrm{He}^{+}$under Simultaneous Irradiation of Fundamental Laser and High-Order Harmonic Pulses," Physical Review Letters 91, 043002 (2003).

[96] K. L. Ishikawa, "Efficient photoemission and ionization of $\mathrm{He}^{+}$by a combined fundamental laser and high-order harmonic pulse," Physical Review A 70, 013412 (2004).

[97] K. Schiessl, E. Persson, A. Scrinzi, and J. Burgdorfer, "Enhancement of high-order harmonic generation by a two-color field: Influence of propagation effects," Physical Review A (Atomic, Molecular, and Optical Physics) 74, 053 412-8 (2006).

[98] M. B. Gaarde, K. J. Schafer, A. Heinrich, J. Biegert, and U. Keller, "Large enhancement of macroscopic yield in attosecond pulse trainassisted harmonic generation," Physical Review A 72, 013411 (2005).

[99] M. M. Fejer, G. A. Magel, D. H. Jundt, and R. L. Byer, "Quasi-phasematched second harmonic generation: tuning and tolerances," Quantum Electronics, IEEE Journal of 28, 2631-2654 (1992).

[100] E. A. Gibson, A. Paul, N. Wagner, R. Tobey, D. Gaudiosi, S. Backus, I. P. Christov, A. Aquila, E. M. Gullikson, D. T. Attwood, M. M. Murnane, and H. C. Kapteyn, "Coherent Soft X-ray Generation in the Water Window with Quasi-Phase Matching," Science 302, 95-98 (2003).

[101] B. H. P. Broks, J. van Dijk, H. M. J. Bastiaens, K.-J. Boller, and J. J. A. M. van der Mullen, "Study of a pulsed capillary discharge waveguide with a modulated radius," Journal of Physics D: Applied Physics 39, 2384 (2006).

[102] M. Zepf, B. Dromey, M. Landreman, P. Foster, and S. M. Hooker, "Bright Quasi-Phase-Matched Soft-X-Ray Harmonic Radiation from Argon Ions," Physical Review Letters 99, 143 901-4 (2007). 
[103] T. Pfeifer and M. C. Downer, "Direct experimental observation of periodic intensity modulation along a straight hollow-core optical waveguide," Journal of the Optical Society of America B 24, 1025-1029 (2007).

[104] S. L. Voronov, I. Kohl, J. B. Madsen, J. Simmons, N. Terry, J. Titensor, Q. Wang, and J. Peatross, "Control of Laser High-Harmonic Generation with Counterpropagating Light," Physical Review Letters 87, 133902 (2001).

[105] O. Cohen, X. Zhang, A. L. Lytle, T. Popmintchev, M. M. Murnane, and H. C. Kapteyn, "Grating-Assisted Phase Matching in Extreme Nonlinear Optics," Physical Review Letters 99, 053 902-4 (2007).

[106] P. Mulser, D. Bauer, and H. Ruhl, "Collisionless Laser-Energy Conversion by Anharmonic Resonance," Physical Review Letters 101, 225002 (2008).

[107] P. Mulser, D. Bauer, S. Hain, H. Ruhl, and F. Cornolti, "Present understanding of superintense laser-solid interaction," Laser Physics 10, $231-240(2000)$.

[108] D. Bauer and P. Mulser, "Vacuum heating versus skin layer absorption of intense femtosecond laser pulses," Physics of Plasmas 14, 023 301-11 (2007).

[109] P. Mora, "Plasma Expansion into a Vacuum," Physical Review Letters 90, 185002 (2003).

[110] E. Racz, I. B. Foldes, G. Kocsis, G. Veres, K. Eidmann, and S. Szatmari, "On the effect of surface rippling on the generation of harmonics in laser plasmas," Applied Physics B: Lasers and Optics 82, 13-18 (2006).

[111] P. McKenna, F. Lindau, O. Lundh, D. Neely, A. Persson, and C.-G. Wahlstrom, "High-intensity laser-driven proton acceleration: influence of pulse contrast," Philosophical Transactions of the Royal Society A: Mathematical, Physical and Engineering Sciences 364, 711-723 (2006).

[112] L. M. Chen, P. Forget, S. Fourmaux, J. C. Kieffer, A. Krol, C. C. Chamberlain, B. X. Hou, J. Nees, and G. Mourou, "Study of hard X-ray emission from intense femtosecond Ti:sapphire laser-solid target interactions," Physics of Plasmas 11, 4439-4445 (2004).

[113] D. Umstadter, "Einstein's impact on optics at the frontier," Physics Letters A 347, 121-132 (2005).

[114] W. L. Kruer, The Physics of Laser Plasma Interactions, Frontiers in Physics (Westview Press, Boulder, Colorado, United States of America, 2003). 
[115] J. Fuchs, P. Antici, E. d'Humieres, E. Lefebvre, M. Borghesi, E. Brambrink, C. A. Cecchetti, M. Kaluza, V. Malka, M. Manclossi, S. Meyroneinc, P. Mora, J. Schreiber, T. Toncian, H. Pepin, and P. Audebert, "Laser-driven proton scaling laws and new paths towards energy increase," Nature Physics 2, 48-54 (2006).

[116] J. Schreiber, Ion Acceleration driven by High-Intensity Laser Pulses, Ph.D. thesis, Muenchen University (2006).

[117] W. Rozmus, V. T. Tikhonchuk, and R. Cauble, "A model of ultrashort laser pulse absorption in solid targets," Physics of Plasmas 3, 360-367 (1996).

[118] T. Y. B. Yang, W. L. Kruer, A. B. Langdon, and T. W. Johnston, "Mechanisms for collisionless absorption of light waves obliquely incident on overdense plasmas with steep density gradients," Physics of Plasmas 3, 2702-2709 (1996).

[119] F. Brunel, "Not-so-resonant, resonant absorption," Physical Review Letters 59, 52 (1987).

[120] F. Quere, C. Thaury, P. Monot, S. Dobosz, P. Martin, J. P. Geindre, and P. Audebert, "Coherent Wake Emission of High-Order Harmonics from Overdense Plasmas," Physical Review Letters 96, 125 004-4 (2006).

[121] L. O. Silva, M. Marti, J. R. Davies, R. A. Fonseca, C. Ren, F. S. Tsung, and W. B. Mori, "Proton Shock Acceleration in Laser-Plasma Interactions," Physical Review Letters 92, 015002 (2004).

[122] E. d'Humieres, E. Lefebvre, L. Gremillet, and V. Malka, "Proton acceleration mechanisms in high-intensity laser interaction with thin foils," Physics of Plasmas 12, 062 704-13 (2005).

[123] L. Yin, B. J. Albright, B. M. Hegelich, K. J. Bowers, K. A. Flippo, T. J. T. Kwan, and J. C. Fernandez, "Monoenergetic and GeV ion acceleration from the laser breakout afterburner using ultrathin targets," Physics of Plasmas 14, 056 706-8 (2007).

[124] T. Esirkepov, M. Borghesi, S. V. Bulanov, G. Mourou, and T. Tajima, "Highly Efficient Relativistic-Ion Generation in the Laser-Piston Regime," Physical Review Letters 92, 175003 (2004).

[125] E. L. Clark, K. Krushelnick, J. R. Davies, M. Zepf, M. Tatarakis, F. N. Beg, A. Machacek, P. A. Norreys, M. I. K. Santala, I. Watts, and A. E. Dangor, "Measurements of Energetic Proton Transport through Magnetized Plasma from Intense Laser Interactions with Solids," Physical Review Letters 84, 670 (2000). 
[126] R. A. Snavely, M. H. Key, S. P. Hatchett, T. E. Cowan, M. Roth, T. W. Phillips, M. A. Stoyer, E. A. Henry, T. C. Sangster, M. S. Singh, S. C. Wilks, A. MacKinnon, A. Offenberger, D. M. Pennington, K. Yasuike, A. B. Langdon, B. F. Lasinski, J. Johnson, M. D. Perry, and E. M. Campbell, "Intense High-Energy Proton Beams from Petawatt-Laser Irradiation of Solids," Physical Review Letters 85, 2945 (2000).

[127] E. L. Clark, K. Krushelnick, M. Zepf, F. N. Beg, M. Tatarakis, A. Machacek, M. I. K. Santala, I. Watts, P. A. Norreys, and A. E. Dangor, "Energetic Heavy-Ion and Proton Generation from Ultraintense Laser-Plasma Interactions with Solids," Physical Review Letters 85, 1654 (2000).

[128] S. P. Hatchett, C. G. Brown, T. E. Cowan, E. A. Henry, J. S. Johnson, M. H. Key, J. A. Koch, A. B. Langdon, B. F. Lasinski, R. W. Lee, A. J. Mackinnon, D. M. Pennington, M. D. Perry, T. W. Phillips, M. Roth, T. C. Sangster, M. S. Singh, R. A. Snavely, M. A. Stoyer, S. C. Wilks, and K. Yasuike, "Electron, photon, and ion beams from the relativistic interaction of Petawatt laser pulses with solid targets," Physics of Plasmas 7, 2076-2082 (2000).

[129] B. M. Hegelich, B. J. Albright, J. Cobble, K. Flippo, S. Letzring, M. Paffett, H. Ruhl, J. Schreiber, R. K. Schulze, and J. C. Fernandez, "Laser acceleration of quasi-monoenergetic $\mathrm{MeV}$ ion beams," Nature 439, 441444 (2006).

[130] M. Kaluza, J. Schreiber, M. I. K. Santala, G. D. Tsakiris, K. Eidmann, J. Meyer-ter Vehn, and K. J. Witte, "Influence of the Laser Prepulse on Proton Acceleration in Thin-Foil Experiments," Physical Review Letters 93, 045 003-4 (2004).

[131] D. Neely, P. Foster, A. Robinson, F. Lindau, O. Lundh, A. Persson, C. G. Wahlstrom, and P. McKenna, "Enhanced proton beams from ultrathin targets driven by high contrast laser pulses," Applied Physics Letters 89, 021 502-3 (2006).

[132] P. Antici, J. Fuchs, E. d'Humieres, E. Lefebvre, M. Borghesi, E. Brambrink, C. A. Cecchetti, S. Gaillard, L. Romagnani, Y. Sentoku, T. Toncian, O. Willi, P. Audebert, and H. Pepin, "Energetic protons generated by ultrahigh contrast laser pulses interacting with ultrathin targets," Physics of Plasmas 14, 030 701-4 (2007).

[133] T. Ceccotti, A. Levy, H. Popescu, F. Reau, P. D'Oliveira, P. Monot, J. P. Geindre, E. Lefebvre, and P. Martin, "Proton Acceleration with HighIntensity Ultrahigh-Contrast Laser Pulses," Physical Review Letters 99, $185002-4$ (2007). 
[134] S. C. Wilks, A. B. Langdon, T. E. Cowan, M. Roth, M. Singh, S. Hatchett, M. H. Key, D. Pennington, A. MacKinnon, and R. A. Snavely, "Energetic proton generation in ultra-intense laser-solid interactions," Physics of Plasmas 8, 542-549 (2001).

[135] M. Allen, Y. Sentoku, P. Audebert, A. Blazevic, T. Cowan, J. Fuchs, J. C. Gauthier, M. Geissel, M. Hegelich, S. Karsch, E. Morse, P. K. Patel, and M. Roth, "Proton spectra from ultraintense laser-plasma interaction with thin foils: Experiments, theory, and simulation," Physics of Plasmas 10, 3283-3289 (2003).

[136] T. Grismayer and P. Mora, "Influence of a finite initial ion density gradient on plasma expansion into a vacuum," Physics of Plasmas 13, 032 1037 (2006).

[137] S. Ter-Avetisyan, M. Schnurer, T. Sokollik, P. V. Nickles, W. Sandner, H. R. Reiss, J. Stein, D. Habs, T. Nakamura, and K. Mima, "Proton acceleration in the electrostatic sheaths of hot electrons governed by strongly relativistic laser-absorption processes," Physical Review E (Statistical, Nonlinear, and Soft Matter Physics) 77, 016 403-5 (2008).

[138] A. J. Mackinnon, Y. Sentoku, P. K. Patel, D. W. Price, S. Hatchett, M. H. Key, C. Andersen, R. Snavely, and R. R. Freeman, "Enhancement of Proton Acceleration by Hot-Electron Recirculation in Thin Foils Irradiated by Ultraintense Laser Pulses," Physical Review Letters 88, 215006 (2002).

[139] Y. Sentoku, T. E. Cowan, A. Kemp, and H. Ruhl, "High energy proton acceleration in interaction of short laser pulse with dense plasma target," Physics of Plasmas 10, 2009-2015 (2003).

[140] P. McKenna, K. W. D. Ledingham, J. M. Yang, L. Robson, T. McCanny, S. Shimizu, R. J. Clarke, D. Neely, K. Spohr, R. Chapman, R. P. Singhal, K. Krushelnick, M. S. Wei, and P. A. Norreys, "Characterization of proton and heavier ion acceleration in ultrahigh-intensity laser interactions with heated target foils," Physical Review E (Statistical, Nonlinear, and Soft Matter Physics) 70, 036 405-6 (2004).

[141] A. Levy, T. Ceccotti, H. Popescu, F. Reau, P. D'Oliveira, P. Monot, P. Martin, J. P. Geindre, and E. Lefebvre, "Proton Acceleration With High-Intensity Laser Pulses in Ultrahigh Contrast Regime," Plasma Science, IEEE Transactions on 36, 1808-1811 (2008).

[142] A. Fukumi, M. Nishiuchi, H. Daido, Z. Li, A. Sagisaka, K. Ogura, S. Orimo, M. Kado, Y. Hayashi, M. Mori, S. V. Bulanov, T. Esirkepov, K. Nemoto, Y. Oishi, T. Nayuki, T. Fujii, A. Noda, and S. Nakamura, "Laser polarization dependence of proton emission from a thin foil target irradiated by a $70 \mathrm{fs}$, intense laser pulse," Physics of Plasmas 12, $100701-4$ (2005). 
[143] S. V. Bulanov, N. M. Naumova, and F. Pegoraro, "Interaction of an ultrashort, relativistically strong laser pulse with an overdense plasma," Physics of Plasmas 1, 745-757 (1994).

[144] R. Lichters, J. Meyer-ter Vehn, and A. Pukhov, "Short-pulse laser harmonics from oscillating plasma surfaces driven at relativistic intensity," Physics of Plasmas 3, 3425-3437 (1996).

[145] D. von der Linde and K. Rzazewski, "High-order optical harmonic generation from solid surfaces," Applied Physics B: Lasers and Optics 63, 499-506 (1996).

[146] P. Gibbon, "Harmonic Generation by Femtosecond Laser-Solid Interaction: A Coherent "Water-Window" Light Source?" Physical Review Letters 76, 50 (1996).

[147] P. A. Norreys, M. Zepf, S. Moustaizis, A. P. Fews, J. Zhang, P. Lee, M. Bakarezos, C. N. Danson, A. Dyson, P. Gibbon, P. Loukakos, D. Neely, F. N. Walsh, J. S. Wark, and A. E. Dangor, "Efficient Extreme UV Harmonics Generated from Picosecond Laser Pulse Interactions with Solid Targets," Physical Review Letters 76, 1832 (1996).

[148] R. Hassner, W. Theobald, S. Niedermeier, H. Schillinger, and R. Sauerbrey, "High-order harmonics from solid targets as a probe for high-density plasmas," Optics Letters 22, 1491-1493 (1997).

[149] M. Zepf, G. D. Tsakiris, G. Pretzler, I. Watts, D. M. Chambers, P. A. Norreys, U. Andiel, A. E. Dangor, K. Eidmann, C. Gahn, A. Machacek, J. S. Wark, and K. Witte, "Role of the plasma scale length in the harmonic generation from solid targets," Physical Review E 58, R5253 (1998).

[150] D. von der Linde, "Generation of high order optical harmonics from solid surfaces," Applied Physics B: Lasers and Optics 68, 315-319 (1999).

[151] A. Tarasevitch, A. Orisch, D. von der Linde, P. Balcou, G. Rey, J. P. Chambaret, U. Teubner, D. Klopfel, and W. Theobald, "Generation of high-order spatially coherent harmonics from solid targets by femtosecond laser pulses," Physical Review A 62, 023816 (2000).

[152] I. Watts, M. Zepf, E. L. Clark, M. Tatarakis, K. Krushelnick, A. E. Dangor, R. M. Allott, R. J. Clarke, D. Neely, and P. A. Norreys, "Dynamics of the Critical Surface in High-Intensity Laser-Solid Interactions: Modulation of the XUV Harmonic Spectra," Physical Review Letters 88, 155001 (2002).

[153] U. Teubner, G. Pretzler, T. Schlegel, K. Eidmann, E. Forster, and K. Witte, "Anomalies in high-order harmonic generation at relativistic intensities," Physical Review A 67, 013816 (2003). 
[154] P. Monot, G. Doumy, S. Dobosz, M. Perdrix, P. D'Oliveira, F. Quere, F. Reau, P. Martin, P. Audebert, J.-C. Gauthier, and J.-P. Geindre, "High-order harmonic generation by nonlinear reflection of an intense high-contrast laser pulse on a plasma," Optics Letters 29, 893-895 (2004).

[155] B. Dromey, M. Zepf, A. Gopal, K. Lancaster, M. S. Wei, K. Krushelnick, M. Tatarakis, N. Vakakis, S. Moustaizis, R. Kodama, M. Tampo, C. Stoeckl, R. Clarke, H. Habara, D. Neely, S. Karsch, and P. Norreys, "High harmonic generation in the relativistic limit," Nature Physics 2, 456-459 (2006).

[156] M. Zepf, B. Dromey, S. Kar, C. Bellei, D. C. Carroll, R. J. Clarke, J. S. Green, S. Kneip, K. Markey, S. R. Nagel, P. T. Simpson, L. Willingale, P. McKenna, D. Neely, Z. Najmudin, K. Krushelnick, and P. A. Norreys, "High harmonics from relativistically oscillating plasma surfaces-a high brightness attosecond source at keV photon energies," Plasma Physics and Controlled Fusion 49, B149-B162 (2007).

[157] R. L. Carman, D. W. Forslund, and J. M. Kindel, "Visible Harmonic Emission as a Way of Measuring Profile Steepening," Physical Review Letters 46, 29 (1981).

[158] S. Kohlweyer, G. D. Tsakiris, C. G. Wahlstrom, C. Tillman, and I. Mercer, "Harmonic generation from solid-vacuum interface irradiated at high laser intensities," Optics Communications 117, 431-438 (1995).

[159] D. von der Linde, T. Engers, G. Jenke, P. Agostini, G. Grillon, E. Nibbering, A. Mysyrowicz, and A. Antonetti, "Generation of high-order harmonics from solid surfaces by intense femtosecond laser pulses," Physical Review A 52, R25 (1995).

[160] U. Teubner, K. Eidmann, U. Wagner, U. Andiel, F. Pisani, G. D. Tsakiris, K. Witte, J. Meyer-ter Vehn, T. Schlegel, and E. Forster, "Harmonic Emission from the Rear Side of Thin Overdense Foils Irradiated with Intense Ultrashort Laser Pulses," Physical Review Letters 92, 185001 (2004).

[161] K. Eidmann, T. Kawachi, A. Marcinkevicius, R. Bartlome, G. D. Tsakiris, K. Witte, and U. Teubner, "Fundamental and harmonic emission from the rear side of a thin overdense foil irradiated by an intense ultrashort laser pulse," Physical Review E 72, 036413 (2005).

[162] A. Tarasevitch, K. Lobov, C. Wunsche, and D. von der Linde, "Transition to the Relativistic Regime in High Order Harmonic Generation," Physical Review Letters 98, 103 902-4 (2007).

[163] B. Dromey, D. Adams, R. Horlein, Y. Nomura, S. G. Rykovanov, D. C. Carroll, P. S. Foster, S. Kar, K. Markey, P. McKenna, D. Neely, 
M. Geissler, G. D. Tsakiris, and M. Zepf, "Diffraction-limited performance and focusing of high harmonics from relativistic plasmas," Nature Physics 5, 146-152 (2009).

[164] M. Rakhmanov, "Reflection of light from a moving mirror: derivation of the relativistic Doppler formula without Lorentz transformations," (2006).

[165] T. Baeva, S. Gordienko, and A. Pukhov, "Theory of high-order harmonic generation in relativistic laser interaction with overdense plasma," Physical Review E (Statistical, Nonlinear, and Soft Matter Physics) 74, 046 404-11 (2006).

[166] B. Dromey, S. Kar, C. Bellei, D. C. Carroll, R. J. Clarke, J. S. Green, S. Kneip, K. Markey, S. R. Nagel, P. T. Simpson, L. Willingale, P. McKenna, D. Neely, Z. Najmudin, K. Krushelnick, P. A. Norreys, and M. Zepf, "Bright Multi-keV Harmonic Generation from Relativistically Oscillating Plasma Surfaces," Physical Review Letters 99, 085 001-4 (2007).

[167] S. Gordienko, A. Pukhov, O. Shorokhov, and T. Baeva, "Relativistic Doppler Effect: Universal Spectra and Zeptosecond Pulses," Physical Review Letters 93, 115002 (2004).

[168] F. Quere, C. Thaury, J. P. Geindre, G. Bonnaud, P. Monot, and P. Martin, "Phase Properties of Laser High-Order Harmonics Generated on Plasma Mirrors," Physical Review Letters 100, 095 004-4 (2008).

[169] P. Gibbon, "High-order harmonic generation in plasmas," Quantum Electronics, IEEE Journal of 33, 1915-1924 (1997).

[170] R. W. Means, L. Muschietti, M. Q. Tran, and J. Vaclavik, "Electromagnetic radiation from an inhomogeneous plasma: Theory and experiment," Physics of Fluids 24, 2197-2207 (1981).

[171] Z.-M. Sheng, K. Mima, J. Zhang, and H. Sanuki, "Emission of Electromagnetic Pulses from Laser Wakefields through Linear Mode Conversion," Physical Review Letters 94, 095003 (2005).

[172] G. Pretzler, A. Kasper, and K. J. Witte, "Angular chirp and tilted light pulses in CPA lasers," Applied Physics B: Lasers and Optics 70, 1-9 (2000).

[173] C. C. de Bruijn, Dynamics of laser produced XUV emitting plasmas, Ph.D. thesis, Technical University of Eindhoven (2004).

[174] P. Salieres, personal Communication. 
[175] E. J. Takahashi, Y. Nabekawa, H. Mashiko, H. Hasegawa, A. Suda, and K. Midorikawa, "Generation of strong optical field in soft X-ray region by using high-order harmonics," Selected Topics in Quantum Electronics, IEEE Journal of 10, 1315-1328 (2004).

[176] C. Thaury, H. George, F. Quere, R. Loch, J. P. Geindre, P. Monot, and P. Martin, "Coherent dynamics of plasma mirrors," Nature Physics 4, 631-634 (2008).

[177] J. O. Stoner Jr and S. Bashkin, "Production of a large unsupported carbon-film ultraviolet filter," Applied Optics 17, 321-325 (1978).

[178] ACF-Metals, "ACF-Metals Product description and technical information," (2005).

[179] J. O. Stoner Jr, "Densities of carbon foils," Nuclear Instruments and Methods in Physics Research Section A: Accelerators, Spectrometers, Detectors and Associated Equipment 303, 94-98 (1991).

[180] A. Schoonderbeek, C. A. Biesheuvel, R. M. Hofstra, K.-J. Boller, and J. Meijer, "The influence of the pulse length on the drilling of metals with an excimer laser," Journal of Laser Applications 16, 85-91 (2004).

[181] C. J. Sofield, C. J. Woods, N. E. B. Cowern, L. B. Bridwell, J. M. Butcher, and J. M. Freeman, "The light element impurity content of selfsupporting carbon foils," Nuclear Instruments and Methods in Physics Research 203, 509-514 (1982).

[182] G. Doumy, S. Dobosz, P. D'Oliveira, P. Monot, M. Perdrix, F. Quere, F. Reau, P. Martin, P. Audebert, J. C. Gauthier, and J. P. Geindre, "High order harmonic generation by non-linear reflection of a pedestalfree intense laser pulse on a plasma," Applied Physics B: Lasers and Optics 78, 901-904 (2004).

[183] R. Weber, J. E. Balmer, and P. Ladrach, "Thomson parabola time-offlight ion spectrometer," Review of Scientific Instruments 57, 1251-1253 (1986).

[184] A. G. Khachatryan, F. A. Van Goor, and K.-J. Boller, "Coherent and incoherent radiation from a channel-guided laser wakefield accelerator," New Journal of Physics 10, 083043 (2008).

[185] H. J. Voorma, E. Louis, F. Bijkerk, and S. Abdali, "Angular and energy dependence of ion bombardment of Mo/Si multilayers," Journal of Applied Physics 82, 1876-1881 (1997).

[186] R. A. Loch, A. Levy, T. Ceccotti, F. Quere, C. Thaury, H. George, F. Bijkerk, K.-J. Boller, and P. Martin, "Enhanced ion acceleration with extremely thin foils," The European Physical Journal Special Topics 175, 133-138 (2009). 
[187] R. A. Loch, T. Ceccotti, F. Quere, H. George, P. Monot, M. Bougeard, F. Reau, P. D'Oliveira, P. Martin, and K.-J. Boller, "Experimental study on proton acceleration in the transparent regime using extremely thin carbon foils," In preparation for "New Journal of Physics".

[188] J. Fuchs, P. Antici, E. d'Humieres, E. Lefebvre, M. Borghesi, E. Brambrink, C. A. Cecchetti, T. Toncian, H. Pepin, and P. Audebert, "Ion acceleration using high-contrast ultra-intense lasers," Journal de Physique IV 133, 1151-1153 (2006).

[189] A. Andreev, A. Levy, T. Ceccotti, C. Thaury, K. Platonov, R. A. Loch, and P. Martin, "Fast-Ion Energy-Flux Enhancement from Ultrathin Foils Irradiated by Intense and High-Contrast Short Laser Pulses," Physical Review Letters 101, 155 002-4 (2008).

[190] B. C. Stuart, M. D. Feit, S. Herman, A. M. Rubenchik, B. W. Shore, and M. D. Perry, "Nanosecond-to-femtosecond laser-induced breakdown in dielectrics," Physical Review B 53, 1749 (1996).

[191] F. Quere, C. Thaury, H. George, J. P. Geindre, E. Lefebvre, G. Bonnaud, S. Hueller, P. Monot, and P. Martin, "Basic mechanisms of laser high-order harmonic generation from plasma mirrors," Journal of Modern Optics 55, $2711-2721$ (2008).

[192] J. Collier, C. Hernandez-Gomez, R. Allott, C. Danson, and A. Hall, "A single-shot third-order autocorrelator for pulse contrast and pulse shape measurements," Laser and Particle Beams 19, 231-235 (2001).

[193] N. Furstenau, "Investigation of laser induced damage, evaporation and ionization with homogeneous inorganic target foils," Fresenius' Journal of Analytical Chemistry 308, 201-205 (1981).

[194] S. Amoruso, G. Ausanio, M. Vitiello, and X. Wang, "Infrared femtosecond laser ablation of graphite in high vacuum probed by optical emission spectroscopy," Applied Physics A: Materials Science \& Processing 81, 981-986 (2005).

[195] P. Gibbon, A. Andreev, E. Lefebvre, G. Bonnaud, H. Ruhl, J. Delettrez, and A. R. Bell, "Calibration of one-dimensional boosted kinetic codes for modeling high-intensity laser-solid interactions," Physics of Plasmas 6, 947-953 (1999).

[196] A. A. Andreev, R. Sonobe, S. Kawata, S. Miyazaki, K. Sakai, K. Miyauchi, T. Kikuchi, K. Platonov, and K. Nemoto, "Effect of a laser prepulse on fast ion generation in the interaction of ultra-short intense laser pulses with a limited-mass foil target," Plasma Physics and Controlled Fusion 48, 1605-1619 (2006). 
[197] R. A. Loch, T. Ceccotti, F. Quere, P. Martin, and K.-J. Boller, "Numerical calculations on the influence of a plasma scale length on ion acceleration in the transparent regime," In preparation.

[198] O. Lundh, F. Lindau, A. Persson, C. G. Wahlstrom, P. McKenna, and D. Batani, "Influence of shock waves on laser-driven proton acceleration," Physical Review E (Statistical, Nonlinear, and Soft Matter Physics) 76, 026 404-8 (2007).

[199] A. A. Andreev, S. Steinke, T. Sokollik, M. Schnurer, S. Ter Avetsiyan, K. Y. Platonov, and P. V. Nickles, "Optimal ion acceleration from ultrathin foils irradiated by a profiled laser pulse of relativistic intensity," Physics of Plasmas 16, 013 103-9 (2009).

[200] A. P. L. Robinson, M. Zepf, S. Kar, R. G. Evans, and C. Bellei, "Radiation pressure acceleration of thin foils with circularly polarized laser pulses," New Journal of Physics 10, 013021 (2008).

[201] M. Huijben, G. Rijnders, D. H. A. Blank, S. Bals, S. V. Aert, J. Verbeeck, G. V. Tendeloo, A. Brinkman, and H. Hilgenkamp, "Electronically coupled complementary interfaces between perovskite band insulators," Nature Materials 5, 556-560 (2006). 
If you don't know where you're going, any road will take you there.

George Harrison (1943 - 2001)

\section{Acknowledgements}

After I finished my master project within the Laser Physics and Nonlinear Optics group at the University of Twente, I was given the opportunity by professor Klaus Boller to do a $\mathrm{PhD}$ in the same group. The exciting project description was basically "you can do anything you want as long as it involves a high-power laser". Having no experience in this field, I started to read a lot of interesting literature. After some time, we made the decision to experimentally investigate the interaction of such a high-power laser with extremely thin foils. Coincidentally, around the same time, during a conference in Salamanca in Spain, I met Philippe Martin, who is the research director of the group Physique à Haute Intensité at CEA Saclay in France. This was the beginning of a successful collaboration on two topics; high-order harmonic generation and ion acceleration. Along the way, another topic crossed the path of my research, namely high harmonic generation in gases.

Having three topics of research and being partly involved in the construction of the terawatt laser at our group, achieving the point of finishing my $\mathrm{PhD}$ felt many times like walking on a very steep road in a high mountain with many different challenges to conquer. Fortunately, I enjoy walking in the mountains, on any road, because the exciting part is that you gain many new perspectives along your path. I am also very fortunate to have met new people along the way and to have been working together with many persons, as it may become apparent that the work presented in this thesis and finishing my $\mathrm{PhD}$ could only be obtained in teamwork. When walking up a steep mountain, however, the danger is that you can easily fall down and don't reach the top. Fortunately, there were many people to prevent me from falling down and push me forward when necessary. Therefore, I would like to take the opportunity to thank everyone who contributed directly or indirectly to the completion of my thesis.

First of all I would like to sincerely thank prof. dr. Klaus Boller, to give me the opportunity to work in this exciting field of physics and to support me during my $\mathrm{PhD}$. Getting the freedom and responsibility to do whatever was necessary was a very educational experience. I also want to thank you for your very wise advices, ever inspiring words and all your many efforts, including those made on my dissertation.

I would also like to express my sincere gratitude to dr. Philippe Martin for giving me the opportunity to collaborate with him and his group to perform experiments at PHI, and for being part of the graduation committee. 
My gratitude also goes to the other members of the graduation committee, prof. dr. Fred Bijkerk, prof. dr. Jennifer Herek and prof. dr. Helmut Zacharias, for kindly agreeing to examine my thesis. I also thank prof. dr. Fred Bijkerk for providing us with transmission gratings and MoSi mirrors.

Special thanks for Arie and Mark with whom I had the most discussions and closest contact. Arie, I greatly enjoyed working together with you in the lab and sharing the same office. And thank you for constructing the laser and making time available for experiments on high harmonic generation. Mark, thank you for the fun and your patience in listening and for the many discussions about all kind of things, including the numerical calculations. And thank you both for being my paranymphs during the defense.

Also my special thanks to Bert Bastiaens for the enjoyable collaboration on the high harmonic generation project and the many advices and help. I am also grateful to Martijn Hendrikx and Bas-Jan Zandt for their large efforts on this project. Without them there would not be a working setup. Also thanks to Hidde Mulder for his assistance. Also thanks to Fred van Goor and Arsen Khachatryan for their help. And I thank Dimitri Geskus and Hein Teunissen for the pleasant work together on the laser. My gratitude also goes to Gerard Oude Meijers for all his help in the lab and on the preparation of the sample holders for the nanofoils and many more constructions. Thanks to Kees Biesheuvel for laser-drilling the micro-holes in my sample holders. Also thanks to Ronald Hagen for writing a script to initiate many numerical calculations after each other, which saved me a lot of time. And thanks to Donna Lee for checking the English of my thesis. I also want to thank all the other members of LPNO for their help through the years.

I am also very grateful to everyone from LPNO and NCLR for being responsible for the daily pleasant working atmosphere and all the fun moments throughout my $\mathrm{PhD}$; for example the many group outings and the holidays with some of you. So also many thanks to: Ab, Anton, Arco, Balaji, Cees, Chris, Claudia, Denny, Edip, Elena, Gerald, Ian, Isabel, Jacob, Jelle, Jeroen, Johan-Martijn, Lars, Leon and Leon, Lilian, Liviu, Marten, Martijn, Olivier, Peter en Peter, Petra, Piet, Ramon, Robert, Roel, Ruud, Simone, Thomas, Willem.

I would also like to express my gratitude to all the members of PHI for their help and the pleasant working atmosphere, and in particular the people with whom I closely collaborated: Fabien, Tiberio, Anna, Cedric, Hervé, Sandrine, Fabrice and Pascal.

There are more people besides the above-mentioned colleagues and friends who have contributed to the spirit in finishing my $\mathrm{PhD}$, and who I would like to thank for all the fun moments: my friends from the photography social club FOTON and from indoor football, and of course thanks to Thijs, Eduard, Karina, Jacco, Marco, Marc, Martijn, Robert, Robert-Paul, Marieke, Bram, Saara-Maarit, Esther, Angela, Mark, Dorleta, Anke, Marije, Nicole, Tom, et al.

Also I have to thank The Beatles; for their uplifting songs are the best 
music to keep a positive spirit.

Of course, also many special thanks to my family and family friends, especially Henny, Ronald, Bert, Arend Jan, Eveline, et al. And last but for sure not least, I would like to use this opportunity to express my deepest gratitude to my parents and brother to whom I dedicate this thesis; mom, dad, Walter, thank you for all your care, fun, love and support.

Rolf

Enschede, September 2009 